Jean-Philippe Garric, Hélène Bocard (dir.)

\title{
Architectes et photographes au xixe siècle
}

Publications de l'Institut national d'histoire de l'art 


\section{Architectes et photographes au XIX ${ }^{\mathrm{e}}$ siècle}

\section{Hélène Bocard et Jean-Philippe Garric (dir.)}

DOI : 10.4000/books.inha.7052

Éditeur : Publications de l'Institut national d'histoire de l'art Année d'édition : 2016

Date de mise en ligne : 5 décembre 2017

Collection: Actes de colloques

EAN électronique : 9782917902622

\section{Sonetation gbooks}

http://books.openedition.org

\section{Référence électronique}

BOCARD, Hélène (dir.) ; GARRIC, Jean-Philippe (dir.). Architectes et photographes au xix siècle. Nouvelle édition [en ligne]. Paris : Publications de l'Institut national d'histoire de l'art, 2016 (généré le 21 septembre 2021). Disponible sur Internet : <http://books.openedition.org/inha/7052>. ISBN : 9782917902622. DOl : https://doi.org/10.4000/books.inha.7052.

Ce document a été généré automatiquement le 21 septembre 2021.

(C) Publications de l'Institut national d'histoire de l'art, 2016

Conditions d'utilisation:

http://www.openedition.org/6540 
Si l'on connaît les relations étroites qui lièrent, au XIX siècle, l'architecture et la photographie, les relations entre l'architecte et le photographe, la façon, notamment, dont le premier apprend rapidement à solliciter et utiliser le second, en influant sur sa pratique, n'ont été abordées que ponctuellement à l'occasion d'études ou d'expositions monographiques. Citons pour mémoire l'exposition « Le photographe et l'architecte. Edouard Baldus, Hector-Martin Lefuel et le chantier du Nouveau Louvre de Napoléon III » (musée du Louvre, 1995) réalisée en grande partie à partir du fonds de l'Agence d'architecture du Louvre, ou des expositions monographiques consacrées à des architectes ("AlfredNicolas Normand», CNMHS, 1978 ou «Charles Garnier, un architecte pour un empire », Paris, ENSBA, 2010). Pourtant, dans leur double volonté de former de vastes corpus visuels pouvant servir de références à leurs projets et de documenter leurs œuvres bâties, y compris dans les phases provisoires de leur mise en œuvre, l'architecte trouve dans le photographe un partenaire décisif, dont il apprendra vite à orienter l'objectif, voire à retoucher ou à recadrer les images, notamment pour les publier.

\section{HÉLÈNE BOCARD}

Hélène Bocard est spécialiste de l'histoire de la photographie du XIX ${ }^{\mathrm{e}}$ siècle.

\section{JEAN-PHILIPPE GARRIC}

Jean-Philippe Garric est professeur à Paris 1 Panthéon-Sorbonne. 
SOMMAIRE

Architectes et photographes au XIX siècle

Anne de Mondenard

L'architecture recadrée: la photographie et le nouveau régime visuel dans la presse

architecturale après 1870

Martin Bressani et Peter Sealy

Gourlier versus Narjoux : le géométral versus la perspective

Labrouste et « l'effet de réel »

L'architecture fragmentée

L'objet architectural comme champ d'expériences visuelles

Photographes et architectes à Rome au XIX siècle

Collaboration et culture partagée, le cas des légendes Chauffourier

Jean-Philippe Garric

Le fonds photographique des architectes Ballu (INHA)

Hélène Bocard

Photographies de voyages

Photographies de travail

Louis Émile Durandelle (1839-1917) : un photographe au service des architectes

Charlotte Leblanc

Fixer, mémoriser la ruine

Enregistrer les étapes de la construction

Promouvoir une œuvre ou une carrière

Architecture et photographie d'architecture au XIX siècle au Portugal

Alexandra Trevisan et Maria Helena Maia

Les rêveries photographiques d'un architecte. La collection Alfred Armand au département des Estampes et de la photographie de la Bibliothèque nationale de France Thomas Cazentre

Alfred Armand (1805-1888), architecte à succès et esthète contrarié

La collection de photographies : composition et constitution

Collection d'architecte ou collection d'esthète?

Avènement du regard photographique sur l'art et le monde

L'album du Santa Lucía

Photographier la perspective urbaine

Amari Peliowski

La restauration du Gros-Horloge de Rouen (1889-1893) par Louis Charles Sauvageot

Un chantier à l'épreuve de la photographie

Sophie Nasi

Au cour de l'histoire communale

Les enjeux d'une restauration

Une restauration symbolique et consensuelle

L'album Durand

Du document d'étude à l'image illustrative

L'usage de la photographie par des architectes

Florent Miane

La restauration de l'église Sainte-Croix

La reconstruction de la tour Saint-Michel

La construction des facultés de médecine et des sciences

Conclusion 


\title{
Architectes et photographes au XIX siècle
}

\author{
Anne de Mondenard
}

1 Restituer le dialogue entre architectes et photographes par le biais d'un autre dialogue, entre historiens de l'architecture et historiens de la photographie, est très stimulant.

2 Cela revient à admettre que la production de photographies représentant des architectures peut être le résultat de collaborations qui engagent l'une et l'autre parties. Le photographe n'est plus considéré comme un exécutant produisant une illustration conforme au point de vue de l'architecte. Cette idée a pourtant largement prévalu dans le domaine de la vue d'architecture où la part d'interprétation du photographe a été toujours plus difficile à identifier.

3 Ce débat agitait, il y a quelques années encore, les chercheurs et les photographes du service de l'Inventaire au ministère de la Culture. Les photographes regrettaient que leurs prises de vues soient envisagées seulement comme l'illustration du travail du chercheur. À l'occasion d'une rencontre, à laquelle j'ai assisté, ils ont même montré à quel point les illustrations demandées ne prenaient pas en compte leurs contraintes propres et devenaient impossibles à réaliser ou, une fois réalisées, n'avaient encore pas grand sens visuel. Ils revendiquaient de pouvoir proposer non pas une illustration mais un point de vue qui serait complémentaire à celui de l'historien de l'architecture.

4 Pour la photographie du XIX siècle, les questions sont plus ou moins les mêmes sauf que les photographes ne sont plus là pour défendre leur point de vue. Il reste leurs photographies, riches d'enseignement toutefois.

5 Les photographies d'architecture du XIX ${ }^{\mathrm{e}}$ siècle ont longtemps été considérées comme des documents anonymes classés par sujets au sein de fonds documentaires. Ces fonds ont été constitués, durant la seconde moitié du XIX ${ }^{\mathrm{e}}$ siècle, dans la foulée de l'invention de la photographie, autant par des administrations que par les architectes eux-mêmes.

$6 \quad$ Il y a une trentaine d'années, les historiens ont commencé à s'intéresser en premier lieu aux fonds que j'appelle administratifs. L'étude des fonds d'architectes est beaucoup plus récente. Moins accessibles car restés en mains privées, ils sont restés plus confidentiels. Les historiens ont donc logiquement commencé leurs recherches en 
consultant des fonds publics, accessibles, riches de centaines de milliers d'épreuves, avant de se pencher sur des fonds, certes importants, mais un peu moins repérés et moins étoffés en volume.

7 Les fonds administratifs, souvent constitués au sein de bibliothèques - bibliothèque de musée, bibliothèque universitaire, bibliothèque d'école, bibliothèque spécialisée --, ont donc offert la première matière pour les historiens.

8 Au moment de leur redécouverte, au début des années 1980, ils étaient oubliés voire abandonnés au sein des institutions. Cet abandon remontait à plusieurs décennies. Classés de façon topographique, ils ont été utilisés jusque dans l'entre-deux-guerres par un public de chercheurs qui trouvaient là, sous forme de tirages originaux, les illustrations de leur propos, la représentation d'une architecture ou des réponses à leurs questions sur l'état d'un bâtiment avant une restauration, ou sur le déroulement d'un chantier de construction.

9 Pourquoi l'entre-deux-guerres signe-t-elle la fin d'une première utilisation de ces fonds? Cette époque est marquée par de grands progrès en matière d'impression d'images. Avoir accès à un tirage original quand on peut retrouver une image équivalente dans un livre ou facilement en commander une reproduction dans une agence photographique, n'est donc plus très pertinent quand on s'intéresse surtout au sujet représenté.

10 En même temps, l'utilisation de ces fonds a conditionné le regard que l'on a porté sur les photographies qui les composaient. L'usage essentiellement documentaire a fait de toutes ces photographies d'architecture des documents, et des documents souvent anonymes. On est parti de l'idée que si les photographies avaient été utilisées comme des documents, c'est qu'elles avaient été produites comme des documents. Autrement dit, le regard du photographe n'était pas une donnée à prendre en compte. Les photographies étaient censées rendre compte d'une réalité perçue comme objective. De l'usage on a déduit une intention. Cette équivalence ne fonctionne pas, comme vous le savez.

11 Les recherches ont fait apparaître une réalité beaucoup plus complexe.

12 Lors d'une première étape, qui est celle de la découverte, on a reconstitué l'histoire de ces fonds (leur origine, leurs modes d'enrichissement) et on a identifié dans la mesure du possible les auteurs des photographies comme Le Secq, Baldus, Marville, Durandelle, Atget. Je cite là les auteurs mis en avant dans la première exposition du fonds de l'École des Beaux-Arts, qui a eu lieu, en $1982^{1}$. Les mêmes auteurs ont continué à être mis en valeur dans d'autres expositions qui ont eu lieu dans les années 1980-1990. C'est un parti pris que j'ai moi-même assumé lors de la présentation du fonds du musée des Monuments français, en $1994^{2}$. Ces expositions ont permis de mettre en valeur la qualité des œuvres et d'identifier des regards de photographes sur l'architecture. Des regards différents du relevé de l'architecte. La vision monumentale d'un Baldus, où il résume un édifice dans une seule image, n'est pas celle d'un Le Secq qui s'intéresse aux rapports des ombres et des lumières sur la pierre. Ces deux auteurs, mais ils ne sont pas les seuls, ont fait l'objet de recherches plus approfondies et ont bénéficié de monographies: Henri Le Secq, en $1986^{3}$, et Edouard Baldus, en $1994^{4}$. Ces travaux de recherches qui voulaient mettre en valeur l'œuvre des photographes cherchaient également à identifier les commanditaires des photographes : des administrations, des compagnies privées ou des architectes. 
13 Baldus, dont l'œuvre s'est nourrie de nombreuses commandes a été un formidable catalyseur pour rapprocher historiens de la photographie et historiens de l'architecture. Dans la monographie qui a été consacrée au photographe, l'historien Barry Bergdoll s'intéressait aux retouches que Baldus pouvait faire sur son négatif pour faire disparaître des éléments qui apparaissaient gênants pour la mise en valeur d'un monument, comme un poteau télégraphique. Il voyait dans les interventions du photographe une façon d'anticiper les travaux de mise en valeur des bâtiments dans l'espace urbain. Les travaux du Second Empire les ont effectivement isolés en les débarrassant de constructions jugées insalubres.

14 L'exposition «Le photographe et l'architecte», qui eut lieu au Louvre en 1995 s'intéressait à la relation entre Baldus et Lefuel à l'occasion de la construction du nouveau Louvre ${ }^{5}$. Elle montrait comment le photographe avait rendu compte du travail de l'architecte, à travers la représentation de l'avancement du chantier, tout en réalisant, lui aussi, une œuvre monumentale. Baldus a saisi l'occasion de cette commande pour produire des tirages de très grand format qui restituaient la monumentalité de l'architecture.

15 Ces recherches ont permis d'instaurer un dialogue constructif entre historiens de la photographie et historiens de l'architecture. Elles ont permis aussi de porter un regard différent sur les fonds de photographies d'architecture. On ne parlait plus de «documents » mais « d'œuvres et de documents ». L'identification d'un nouvel auteur permettant à chaque fois d'augmenter le volume des œuvres par rapport aux documents.

16 Je voudrais apporter une nuance à cette classification. J'ai compulsé des dizaines de milliers de photographies d'architecture. Je me suis aperçue que plus le photographe proposait un point de vue inédit ou une composition intéressante, plus j'avais envie de m'intéresser au sujet représenté. Je me suis attardée sur ces œuvres qui invitent à voir un sujet. Je suis passée beaucoup plus vite sur des photographies qualifiées de documentaires mais que l'on regarde sans voir.

17 L'objet de cette journée d'étude n'est pas d'entrer dans ce débat mais de s'intéresser à la nature des relations entre architectes et photographes au XIX siècle. Cela paraît effectivement essentiel de mieux comprendre la demande de l'architecte et la façon dont le photographe y répond, pour remettre en contexte la production et les usages.

18 Les fonds d'architectes, qui font aujourd'hui l'objet d'études approfondies, devraient apporter de nouveaux éclairages en même temps qu'ils ouvrent de nouvelles questions.

19 Dans quel but les architectes ont-ils rassemblé des photographies?

20 S'agit-il d'un outil de documentation pour nourrir leur inspiration, justifier d'un état d'un bâtiment en vue d'une intervention? Ou s'agit-il de représentations de leurs réalisations architecturales. Mais dans quel but? Pour en garder une trace ou pour en assurer la promotion? Comment ont-ils rassemblé ces photographies? Ont-ils acheté des épreuves disponibles ou en sont-ils les commanditaires?

21 Ces représentations architecturales étant vues à travers un autre œil, celui du photographe, on peut encore se demander jusqu'où l'architecte guide-t-il le photographe.

22 Que lui impose-t-il ? Un point de vue, des interventions postérieures à la prise de vue, sur le négatif ou sur l'épreuve? 
23 Il s'agit bien d'une relation où chacun joue un rôle mais où chacun est aussi dépendant de l'autre: le photographe de l'architecte, pour réaliser ses prises de vues, et l'architecte du photographe, pour assurer la représentation de son œuvre.

24 La place du photographe n'étant plus reléguée à celle d'un producteur de documents vides d'interprétation, il devient plus intéressant de se pencher sur la nature des relations qui se sont tissées entre ces deux professions. Une relation qui fait intervenir plusieurs acteurs, l'architecte et le photographe, certes, mais aussi un commanditaire et un utilisateur qui ne se confondent pas nécessairement et peuvent être différents de l'architecte.

25 C'est dans cette tension entre la demande, la réalisation et l'utilisation que se situe le champ riche, qu'explorent les articles que l'on va lire et que nous devons pour certains à des historiens de l'architecture, pour d'autres à des historiens de la photographie.

\section{NOTES}

1. La photographie comme modèle, aperçu du fonds de photographies anciennes de l'École des Beaux-Arts, Paris, ENSBA, 1982.

2. Photographier l'architecture : 1851-1920, collection du musée des Monuments français, Paris, Réunion des musées nationaux, 1994.

3. Henri Le Secq, photographe de 1850 à 1860. Catalogue raisonné de la collection de la bibliothèque des arts décoratifs, Paris, Flammarion, 1986.

4. Edouard Baldus, photographe, New York, Metropolitan Museum of Art, Montréal, Centre canadien d'architecture, Paris, musée des Monuments français, Réunion des musées nationaux, 1994.

5. Le photographe et l'architecte: Édouard Baldus, Hector-Martin Lefuel et le chantier du nouveau Louvre de Napoléon III, Paris, Musée du Louvre/Réunion des musées nationaux, 1995.

\section{INDEX}

Mots-clés : architecte, architecture, photographie

Thèmes : architecture, photographie

Index chronologique : XIXe siècle 


\title{
L'architecture recadrée : la photographie et le nouveau régime visuel dans la presse architecturale après 1870
}

\author{
Martin Bressani et Peter Sealy
}

\section{Gourlier versus Narjoux : le géométral versus la perspective}

1 Le mode de représentation architecturale, tel qu'il fut pratiqué par les architectes, a subi des transformations importantes en France tout au long du xix siècle. Comparons, par exemple, deux importants recueils de monuments français et parisiens du début et de la fin de ce siècle. Le Choix d'édifices publics de Charles Pierre Gourlier est publié entre 1825 et 1850 et répertorie en trois gros volumes 209 édifices publics, choisis à partir des archives du Conseil des bâtiments civils ${ }^{1}$. Les 389 planches gravées ont été produites en grande partie à partir des dessins des architectes et ne montrent aucune vue en trois dimensions, le dessin géométral étant le seul utilisé. Chaque bâtiment est illustré par une à six planches - plans, coupes, élévations et détails à petite échelle - une représentation appropriée pour ces édifices souvent d'un néoclassicisme simplifié. Dans son rapport fait au ministre de l'Intérieur au sujet de cette publication, l'Académie Royale des Beaux-Arts déclare: "Ici, rien n'est idéal, tout au contraire est réel, positif ${ }^{2}$. Cette réalité positive de la pratique architecturale en France est convenablement traduite par la représentation géométrale.

2 Tournons-nous maintenant vers Paris, Monuments élevés par la ville de Félix Narjoux, publié entre 1880 et $1883^{3}$. L'ouvrage ne compte pas moins de 148 vues perspectives pour l'ensemble des quatre volumes. Souvent, un seul bâtiment peut faire l'objet de plusieurs vues, jusqu'à dix-huit dans le cas exceptionnel du Palais de justice. Les vues perspectives sont plus fréquentes dans les figures insérées dans le texte, mais 73 des 
292 grandes planches gravées sont aussi des perspectives, sans compter celles à vol d'oiseau. Les dessins géométraux demeurent majoritaires, mais rare est l'édifice qui n'est pas représenté par au moins une perspective.

3 Cette comparaison entre les recueils de Gourlier et Narjoux illustre un important changement dans le régime visuel des architectes entre la première moitié du siècle et les années 1880, l'image prenant le dessus sur les représentations plus abstraites. La question est de savoir quel rôle la photographie a pu jouer dans cette mutation. Le recueil de Narjoux n'inclut aucune photographie, un mode de représentation qui pourtant en 1880 commençait à prendre sa place dans ce genre de publication grâce aux procédés de photogravure, de demi-ton, d'héliogravure, etc. Mais on y sent une auscultation de l'objet architectural qui est propre à la photographie.

4 Il est entendu que le regain de faveur des représentations en perspective chez les architectes au cours du xix siècle ne tient pas seulement à l'émergence de la photographie. Il reflète soit un goût nouveau pour les complexités spatiales inspirées $\mathrm{du}$ baroque, soit une nouvelle perception dynamique de l'objet architectural ${ }^{4}$. Mais nous croyons que la photographie a aussi joué son rôle, particulièrement après 1870 . N'ayant pas conduit de recherches d'archives encore assez détaillées, nos conclusions resteront préliminaires. Mais nous croyons néanmoins pouvoir démontrer, à l'aide d'une analyse visuelle simple, comment, à partir des années 1870, la représentation des architectes était médiatisée par l'image photographique.

5 Comme nous l'avions déjà souligné dans notre article sur Charles Garnier et la photographie $^{5}$, le dessin en perspective est une forme de représentation généralement défavorisée dans la tradition des beaux-arts, tradition qui privilégiait presque exclusivement le plan, la coupe et l'élévation pour la représentation des nouveaux bâtiments. Il y a plusieurs exceptions, mais de façon générale la prédominance du géométral est écrasante. Les dessins pour les Grands Prix de Rome illustrent parfaitement cet état de chose: malgré toute l'attention donnée à ces machines grandioses dessinées sur une période de plusieurs mois, jamais une perspective ne fut requise avant le Grand Prix de 1863. Dans la presse architecturale, on trouve la même prédominance du géométral, la perspective étant réservée presque exclusivement aux objets décoratifs - fontaines, kiosques, pavillons - ou aux monuments historiques. Pour un nouveau bâtiment, ce sont toujours les dessins de l'architecte en géométral qui sont gravés. Il s'agit là d'un parti pris propre au néoclassicisme français, reflet d'une conception classique et rationaliste du projet tel que le codifiera Durand. Il fallut attendre les efforts de Garnier et de Viollet-le-Duc pour qu'une conception plus dynamique et plus subjective de l'espace architectural sollicite une représentation plus variée et tridimensionnelle. César Daly lui-même introduisit la perspective progressivement dans les pages de sa Revue générale ${ }^{6}$.

6 Mais rappelons que notre objet n'est pas tant de retracer le retour de l'image tridimensionnelle en soi, mais bien l'émergence d'un régime visuel que l'on pourrait qualifier de "photo-scopique », c'est-à-dire une appréhension de l'objet architectural en trois dimensions, médiatisée par l'appareil photographique. 


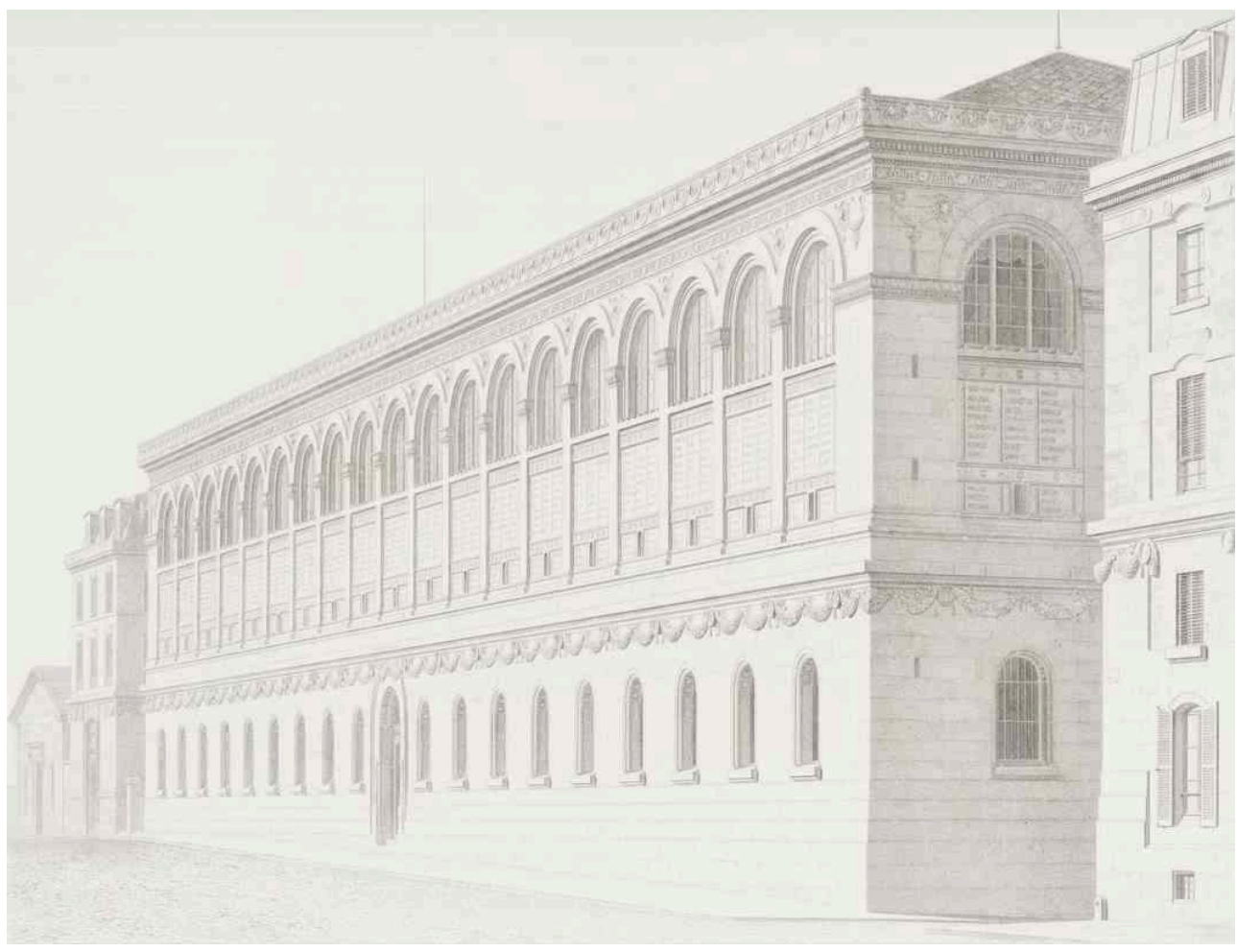

Fig. 1 : Bibliothèque Sainte-Geneviève, à Paris. Vue perspective de l'ensemble de la façade principale, Revue générale de l'architecture et des travaux publics, vol. 11, 1853, pl. 31.

\section{Labrouste et « l'effet de réel »}

Un exemple précis nous permettra d'aiguiller la réflexion sur cet angle de la question. En 1853, César Daly publie dans sa Revue générale une gravure qui illustre la célèbre bibliothèque Sainte-Geneviève en perspective [fig. 1]. Grâce aux travaux de Neil Levine, entre autres, un article qu'il a publié en 2012, nous savons que ce dessin gravé par Jacques Joseph Huguenet fut tracé par Labrouste à partir d'une photographie des frères Bisson'. Il s'agit là, nous apprend Levine, du premier exemple connu d'un dessin architectural publié basé sur une photographie prise à cette fin $^{8}$. Levine a aussi démontré de façon convaincante que ce choix n'était pas qu'un simple expédient pratique mais une décision délibérée de la part de Labrouste: d'une part, tirer une épreuve photographique sur papier salé d'après un négatif n'était rien moins que simple en 1852 ; d'autre part, pour un dessinateur aussi talentueux que Labrouste, il aurait été aussi facile, sinon plus, de dessiner cette perspective sans l'aide d'une photo. Selon Levine, le but de l'architecte était avant tout d'ordre conceptuel plutôt que technique. Il cherchait à dépasser l'aspect trompeur du dessin en perspective, une forme de représentation que son ami César Daly décrivait à l'époque comme étant « un art des illusions" où "constamment l'apparence contredit la réalité9." Avec la photographie, Labrouste se donnait un gage de réalisme quasi mécanique, atteignant une objectivité brute. Levine insiste sur le caractère " anti-subjectif ", clinique et même étrange du dessin de Labrouste tel que gravé par Huguenet. Nous sommes d'accord avec tous ces qualificatifs. On imagine très bien le plaisir que Labrouste ait pu avoir à recourir à une photographie pour générer un "effet de réel» pour reprendre l'expression de Barthes : une image qui apparaît en deçà du subjectif, et qui évite ainsi 
les pièges de la représentation. L'aspect fantomatique du "moule " photographique, comme on décrivait si souvent la nouvelle technologie à l'époque, s'accorde parfaitement avec la vision romantique de Labrouste qui est à la recherche de l'absolu et du primordial. Le dessin de Labrouste accentue d'ailleurs cet aspect étrange de la photographie. Tout ce qui donnait un caractère plus contingent ou plus vivant à la photo est éliminé : l'ombre du Panthéon sur la façade est effacée, les lampadaires qui faisaient irruption au premier plan sont biffés, les personnages qui, ici et là, vaquaient à leurs occupations, sont supprimés. Il crée ainsi une image sans le moindre bruit visuel, d'un silence presque funèbre.

8 Un tel dépouillement dans la représentation n'était pas le seul apanage de Labrouste, ni nécessairement dépendant d'une armature photographique. On retrouve le même ascétisme dans les magnifiques perspectives de l'École des Beaux-Arts que Félix Duban a dessiné pour l'album d'Orléans ${ }^{10}$. Cet album était un cadeau princier destiné au futur Frédéric Guillaume IV, roi de Prusse, un amateur d'art qui avait connu Percier et qui était très proche de Schinkel. L'austérité du dessin de Duban n'est pas sans rappeler les gravures de la Sammlung de Schinkel ou certains dessins de Percier. Il existait en France une tradition du dessin austère et clinique, particulièrement pour la gravure. L'immense ouvrage sur Rome de Paul Marie Letarouilly peut fournir un exemple contemporain de Labrouste. Même si Letarouilly insère parfois quelques personnages pour ajouter une couleur locale, son dessin, surtout une fois gravé, reste froid et objectif.

Il est très possible que les frères Bisson se soient eux-mêmes inspirés de ce genre de représentations pour leur photographie d'architecture. Marie-Noëlle Leroy, dans un article de 1997, explique comment, à partir de 1852, l'année même où ils ont fait leur cliché de la bibliothèque Sainte-Geneviève, les frères Bisson cherchent à pénétrer, en s'y distinguant, le marché de la photographie d'architecture. Elle caractérise leur travail comme privilégiant les vues serrées, «mettant en évidence la façade, évitant ainsi de distraire l'attention ${ }^{11} »$. Leroy note qu'en écartant la vision frontale, Bisson « obtient une sensation de profondeur beaucoup plus importante, tout en donnant plus d'informations ${ }^{12}$ ». Leur photographie de la bibliothèque Sainte-Geneviève correspond à ce schéma. Ils ont positionné leur camera au seul endroit possible pour capter l'entièreté de la façade principale. La vue à angle serré met l'emphase sur la bibliothèque en tant qu'objet unifié et prismatique. Les transformations faites lors $\mathrm{du}$ passage de la photographie à la gravure ne font qu'augmenter ces qualités. Si la photographie offre une égalité de détail, la gravure, elle, renforce les hiérarchies : les lignes des profils ont reçu une légère emphase, les noms inscrits sur la façade sont moins flous. Le résultat est une image idéalisée, typique de la gravure.

En somme, la photographie fournit à Labrouste le gage du réalisme associé à une reproduction "mécanique », mais elle ne modifie en rien son point de vue. D’ailleurs, un rapide croquis fait par Labrouste en 1839 révèle un point de vue semblable, produit par l'architecte longtemps avant la photographie des frères Bisson ${ }^{13}$. Comme l'a si bien démontré Levine, la photographie n'a fait que confirmer la vision de l'architecte sans la modifier. 


\section{L'architecture fragmentée}

11 Nous aimerions maintenant montrer un exemple où la photographie amène définitivement un nouveau point de vue, le cadrage de l'appareil photographique causant, de façon tout à fait délibérée, une fragmentation de l'objet architectural. Il s'agit par contre d'un cas plutôt exceptionnel: la maison pompéienne du prince Napoléon construite sur l'avenue Montaigne à Paris entre 1856 et 1860 . Son architecte, Alfred Nicolas Normand, Prix de Rome de 1846, était également photographe. Il faut attendre 1891, soit l'année précédant la démolition du bâtiment, pour qu'il photographie la maison pompéienne. Cependant, cette maison si singulière avait fait l'objet de plusieurs campagnes photographiques au moment de sa construction. On connaît des photographies par Bingham, Godefroy, Richebourg et Laplanche. C'est celles que ce dernier a réalisées autour de 1866 qui ont retenu notre attention. Il s'agit d'un album réuni sous le titre de Monographie de la maison gréco-romaine construite pour sa majesté impérial Mgrle prince Napoléon par M. Alfred Normand et photographié par J. Laplanche, contenant un plan et vingt et une épreuves sur papier albuminé d'après négatif sur verre ${ }^{14}$. Il a été impossible de déterminer si l'album était une commande ou s'il s'agissait d'une initiative de Laplanche lui-même. La date de 1866 laisse plutôt croire qu'il fut commandé par le groupe qui, cette année-là, avait acheté la maison pour la transformer en un musée d'antiques. Quoiqu'il en soit, l'album est remarquable à plus d'un égard. Notons d'abord que la suite d'images constitue un rare exemple d'exploration photographique systématique, formant une promenade architecturale à travers le bâtiment de l'extérieur à l'intérieur que le lecteur peut suivre grâce à un plan gravé inséré dans l'album. La promenade débute à l'extérieur avec des photographies de la cour et du portique d'entrée. Les images suivantes explorent l'atrium central et les salles avoisinantes sous plusieurs angles. Finalement, cette visite photographique s'achève avec plusieurs vues des jardins d'hiver et de la cour arrière.

L'album se veut donc une sorte de résumé d'une expérience architecturale, plutôt qu'un dossier qui documente objectivement un bâtiment. Les contrastes de lumière parfois très prononcés mettent en évidence le désir de transmettre une impression plutôt que de constituer une documentation. La maison pompéienne est peuplée d'ombre, y circuler consiste à suivre un parcours archéologique d'ordre presque initiatique. 


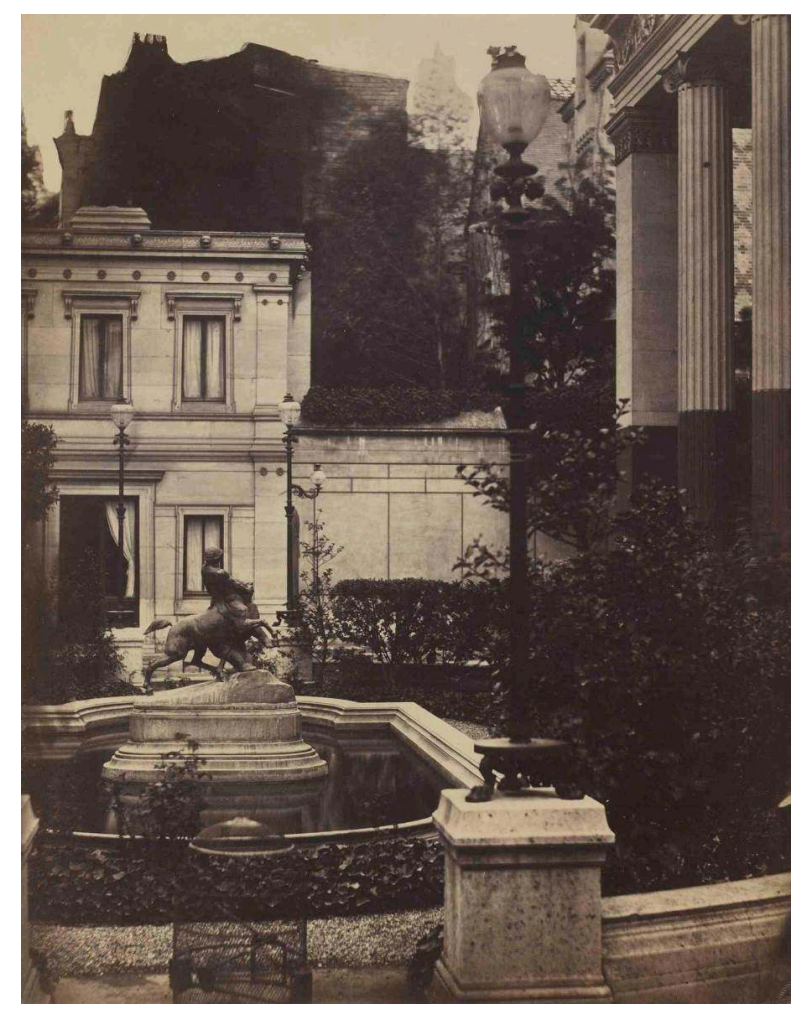

Fig. 2 : Vue de la cour avant, Monographie de la maison gréco-romaine construite pour sa majesté impérial Mgr le prince Napoléon par M. Alfred Normand et photographiée par J. Laplanche, Paris, J. Laplanche et Cie., s. d.

Malgré ce parcours progressif et linéaire, le cadrage photographique n'insiste pas sur l'unité de l'objet architectural. On sent la liberté de l'œil photographique qui n'hésite pas à décentrer l'image et à fragmenter l'objet. Comparons les photos de Laplanche au célèbre tableau de Gustave Boulanger, La répétition du joueur de flûte et de la femme de Diomède chez le prince Napoléon dans l'atrium de sa maison pompéienne, de $1860^{15}$. Cette image centre la composition sur l'atrium central et présente ainsi la maison comme un ensemble unifié : le dessin résume la maison entière. Les épreuves de Laplanche, au contraire, morcellent l'espace en une série de vues partielles, que ce soit dû à une trop grande quantité d'ombre ou, de façon plus décisive, au point de vue adopté [fig. 2]. Il met le regard du spectateur au centre de la composition. En capturant l'impression fugitive, momentanée, le photographe déstabilise l'ensemble architectural.

La maison pompéienne était bien sûr une curiosité, et rien n'indique que Laplanche ait visé avec son album un auditoire d'architectes. On imagine plutôt qu'il comptait sur un public de curieux, d'amateurs de musées et d'antiquités. Même s'il n'a pas influencé les architectes, son album n'en reste pas moins intéressant pour illustrer comment la photographie peut restructurer la vision d'un bâtiment. La photographie stimule un désir scopique, amenant un nouveau modèle optique. Il n'est pas nécessaire d'attendre le Bauhaus et Moholy-Nagy pour qu'un recadrage du monde par la photographie se constitue. 


\section{L'objet architectural comme champ d'expériences visuelles}

15 Afin d'appuyer cette thèse, nous terminerons sur quelques exemples tirés de la presse architecturale des années 1870, entre autre la Revue générale de l'architecture de César Daly et la Gazette des architectes et des bâtiments sous le contrôle de Viollet-le-Duc. Nous analyserons cette fois des gravures, mais des gravures qui, selon nous, portent la marque d'une médiation photographique. Contrairement au cas de la bibliothèque Sainte-Geneviève, nous n'avons cependant aucun document prouvant que celles-ci aient été tracées à partir de photographies. Notre analyse reste donc spéculative. Mais signalons que, dans les années 1870 , les gravures faites à partir de photographies sont de plus en plus communes; on le sait car plusieurs sont identifiées comme telles, le titre portant la mention « d'après une photographie ${ }^{16}$ ». Et, grâce à Neil Levine, on sait que, dans certains cas, elles sont tracées d'après des photographies sans que cela soit indiqué ${ }^{17}$.

16 Aucun des exemples de gravures que nous présenterons ne porte l'indication « d'après une photographie ", mais la présence cachée de l'appareil photographique nous semble potentiellement encore plus intéressante. Car cette pulsion scopique que nous cherchons à identifier, ce besoin de voir et ce désir de regarder qui transforme l'objet architectural en un champ infini d'expériences visuelles, n'est évidemment pas entièrement dépendant de l'appareil photographique. Celui-ci crée et stimule ce désir, mais cet appétit peut vivre de sa vie propre.

17 La Revue générale de César Daly a publié des vues perspectives avant 1870 - nous avons vu l'exemple précoce de la bibliothèque Sainte-Geneviève. Il y aura aussi des vues perspectives de l'hôtel de M. Fould par Labrouste publiées en 1858 et une série pour les projets du nouvel opéra de Paris $^{18}$. Mais ces exemples restent des exceptions. 


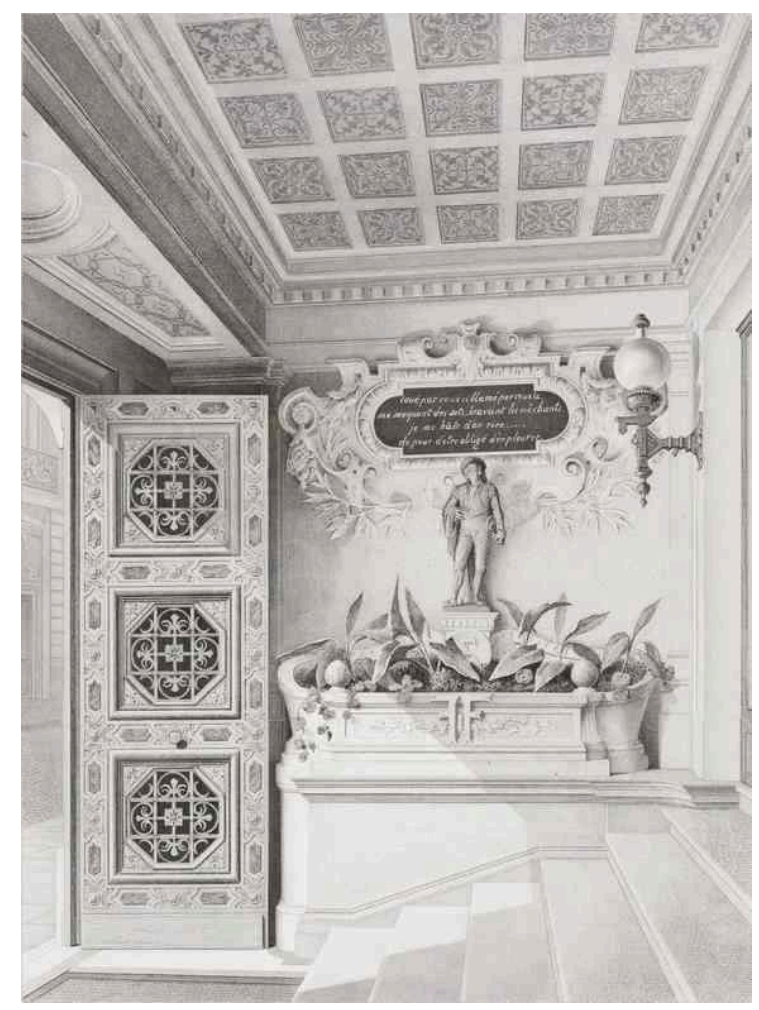

Fig. 3 : Hôtel du Figaro à Paris. Vestibule d'entrée, Revue générale de l'architecture et des travaux publics, vol. 31,1874 , pl. 48.

C'est l'année 1872 qui marque une transformation importante et même un point tournant. Entre 1861 et 1871, Daly publie 28 planches en perspective ; soit, en moyenne, moins de trois par volume. Par contre, dans les dix volumes parus entre 1872 et 1881 , on trouve 102 perspectives, soit une moyenne supérieure à dix par volume. Ces perspectives prennent plusieurs formes : certaines respectent l'unité du bâtiment en offrant une vue de l'objet dans son contexte, soit pittoresque, soit urbain, tandis que d'autres représentent des sujets historiques ${ }^{19}$. Mais un certain nombre se démarquent par la façon inusitée dont elles recadrent l'objet architectural. Nous choisirons deux exemples. En 1874, la Revue générale publia six planches de l'hôtel du journal Le Figaro, conçu par l'architecte Aimé Sauffroy. Trois de ces planches sont des perspectives. Notons d'abord le choix de montrer la façade principale en perspective avec une vue légèrement asymétrique et non par une élévation géométrale ${ }^{20}$. On perçoit même le léger étirement vertical causé par la correction de la perspective grâce au mouvement de bascule du soufflet de la chambre photographique. La perspective de la salle du public n'a rien de très remarquable, sauf peut-être le degré de précision et l'égalité des détails ${ }^{21}$. La vue du vestibule d'entrée, par contre, est étonnante [fig. 3]. Non seulement la qualité du clair-obscur est remarquable, donnant une modularité tridimensionnelle à l'espace, mais le cadrage est vraiment singulier, donnant l'impression d'une photographie instantanée plutôt qu'un dessin délibéré et réfléchi.

Si les gravures de l'hôtel du Figaro donnent malgré tout une vision plutôt unifiée et somme toute conventionnelle du bâtiment à travers ses principaux espaces, un deuxième exemple témoigne d'un regard plus exploratoire, qui semble fureter dans l'édifice. En 1872, la Revue générale publie deux perspectives du nouveau bâtiment du ministère de l'Agriculture, du Commerce et des Travaux publics d'Antoine Isidore Eugène Godeboeuf à Paris. La première, une vue intérieure, est une composition plutôt 
abstraite montrant l'intersection d'un passage intérieur et l'entrée ${ }^{22}$. Cette vue explore un fragment d'espace. Ici aussi, les ombres, parfaitement modelées, accentuent l'effet de profondeur. Le niveau de détail est égal à travers l'image. Une deuxième perspective, très semblable, nous montre un espace plus complexe, comprenant simultanément la voûte du passage et la cour intérieure ${ }^{23}$. On y sent presque l'effet de parallaxe. Ces deux perspectives sont d'autant plus étonnantes, que ce sont les seules publiées par Daly de cet imposant édifice dessiné par Godeboeuf. On n'y trouve aucune vue générale extérieure ou intérieure. Les deux images semblent répondre à une curiosité, le plaisir d'une pénétration visuelle et dynamique de l'espace, plutôt qu'au souci de comprendre un ensemble unifié.

Malgré la très grande variété d'images présentées par la Revue générale dans les années 1870 et 1880 , nous pouvons faire une observation générale. Ce qui unit les perspectives plus traditionnelles à celles plus radicales, c'est l'idée que le bâtiment est devenu un champ d'expérience visuel, à saisir par des vues variées, c'est-à-dire des moments dans le temps et dans l'espace. Certaines de ces vues donnent l'image d'un objet unifié, d'autres d'un fragment libéré de son contexte.

21 Si la Revue générale de Daly représente une certaine orthodoxie dans le monde architectural du Second Empire, la Gazette des architectes et du bâtiment, contrôlée par les Viollet-le-Duc, se voulait à l'avant-garde ${ }^{24}$. Durant sa première période, la Gazette s'est particulièrement attachée à la question de la représentation architecturale. Dans son «Article préliminaire », Viollet-le-Duc fils déclare que les illustrations sont «bien plus encore que le texte, la démonstration de l'objet figuré, et viennent de cette manière audevant des explications ${ }^{25} \%$. Souvent préférée au texte, l'image occupe une place dominante et fait l'objet de beaucoup d'expérimentation. Ce n'est pas un hasard si un des articles majeurs de cette revue est entièrement consacré au dessin : le « Nouveau traité de perspective » de l'esthéticien suisse David Sutter, paru en feuilletons répartis sur trois années. Il s'agit d'une étude hautement scientifique de l'art de la perspective, basée sur l'analyse détaillée de la physiologie de l'œil, décrivant les mécanismes de perception optique. Sutter cherche à établir des règles positives pour le dessin et la peinture. « La loi du tableau », écrit-il, « c'est la loi de l'œil ${ }^{26}$ ».

22 Toutes les images parues dans la Gazette de 1863 à 1871 furent gravées sur des plaques de cuivre selon le nouveau procédé de Xavier Comte. Moins chère que la gravure en creux, cette technique dérivée de la lithographie offrait surtout la possibilité d'imprimer texte et image simultanément ${ }^{27}$. Les illustrations de la Gazette ont assumé un rôle explicatif, dans le but de faire comprendre le fonctionnement des objets et leur construction. On y trouve même une vue axonométrique quelques décennies avant Choisy ${ }^{28}$. Ce fut d'ailleurs dans les pages de la Gazette, que Choisy écrivit un de ses premiers articles.

Mais l'illustration qui attire notre attention sur la Gazette est toute autre. À travers les années 1868-1871, la Gazette a publié un long article en plusieurs livraisons sur la maison du couturier Charles Frédéric Worth à Suresnes, restaurée et agrandie par l'architecte Denis Darcy, un disciple de Viollet-le-Duc ${ }^{29}$. Le texte, rédigé par Anatole de Baudot, était accompagné d'un très grand nombre de dessins en géométral et surtout en perspective. Des 43 figures publiées sur le bâtiment, 18 sont des vues. Une vue à vol d'oiseau permet de comprendre l'ensemble assez complexe ${ }^{30}$, mais le plus grand nombre sont des vues partielles, formant une sorte de collage d'éléments divers ${ }^{31}$. Dans sa «notice descriptive», Baudot remarque: «L'habitation dont il s'agit n'a pas été 
conçue sur un plan d'ensemble, et n'est que le résultat d'adjonctions et de transformations successives. Elle est néanmoins fort intéressante à divers points de vue... ${ }^{32} »$. Cet amalgame forme un champ idéal pour un travail de recadrage par le biais de la photographie - même si nous n'avons aucun document prouvant l'utilisation de cet intermédiaire.

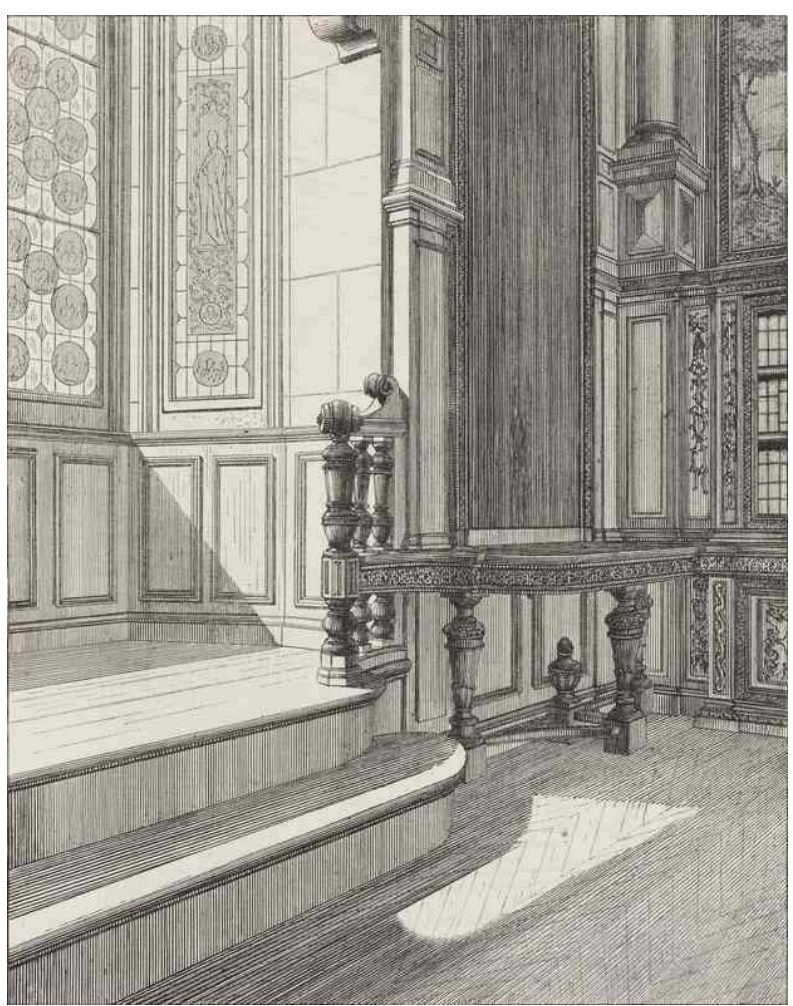

Fig. 4 : Propriété de M. W..., à Suresnes (Seine). Bibliothèque. Perspective, Gazette des architectes et du bâtiment, vol. 7, 1869-1871, fig. 91. 


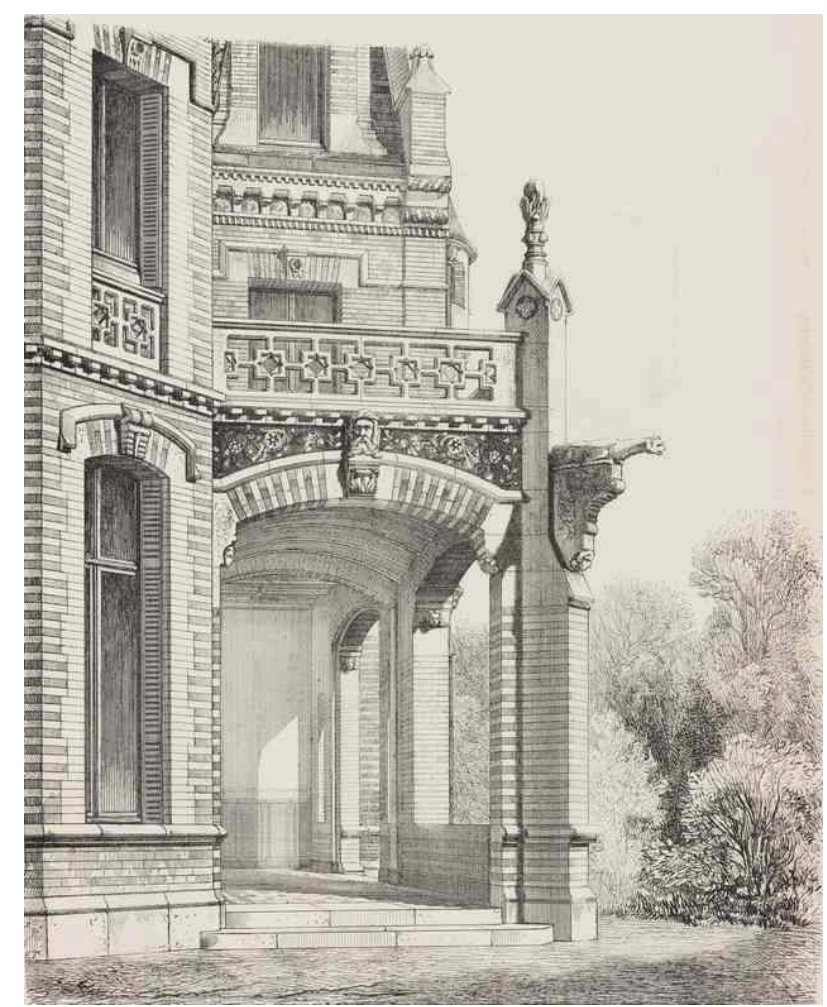

Fig. 5 : Villa Honoré à Trouville-sur-mer. Perspective du porche, Gazette des architectes et du bâtiment, vol. 7, 1869-1871, fig. 201.

Si la Monographie de la maison gréco-romaine de Laplanche formait un parcours linéaire à travers la maison pompéienne, les images de la maison Worth ne suivent pas une telle logique. L'objectif fouille l'espace selon la curiosité du promeneur [fig.4]. Nous trouvons la même liberté d'exploration dans une série de vues perspectives publiée dans la Gazette en 1869-1870 de la villa Honoré à Trouville dessinée par l'architecte Devrez $^{33}$. Cette fois, c'est le volume extérieur qui fait l'objet d'une exploration visuelle. Ce qui est particulièrement remarquable, c'est que chacune des vues est partielle, presque oblique [fig. 5]. 


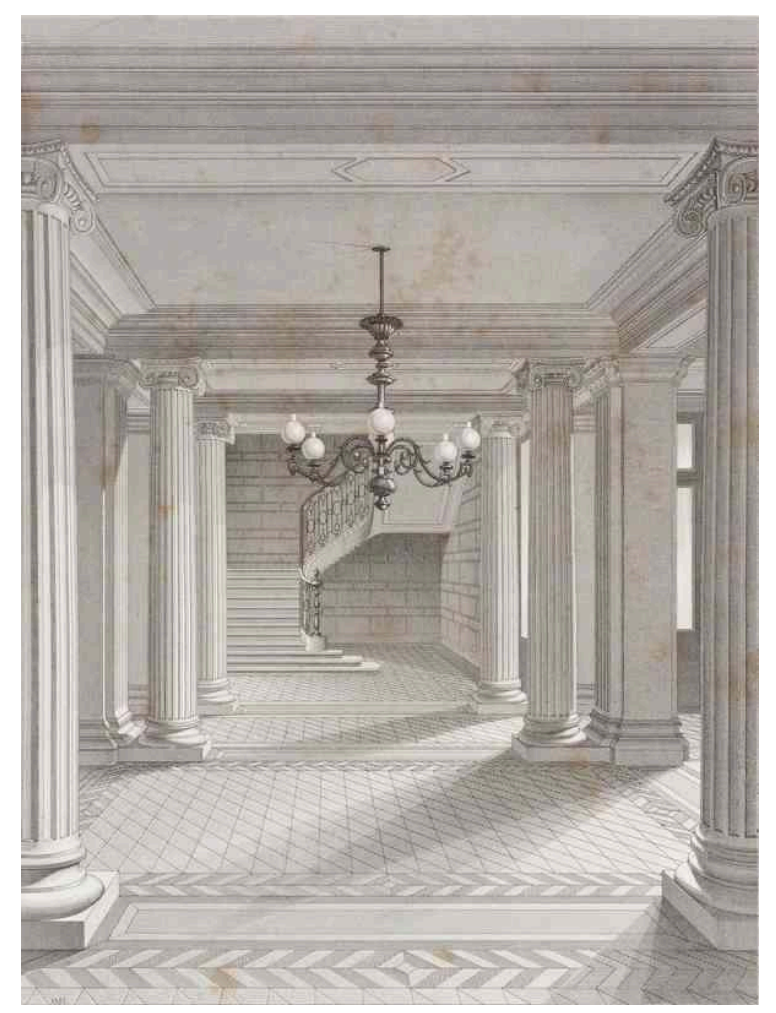

Fig. 6 : Lycée Saint-Louis. Vestibule. III, Félix Narjoux, Paris, monuments élevés par la ville, 1850-1880, vol. II, Paris, Vve. A. Morel et Cie., 1880-1883.

Nous allons conclure sur un bref retour sur la publication de Félix Narjoux avec laquelle nous avons ouvert notre communication. La variété de représentations déployées dans cet ouvrage est vraiment remarquable, dont de très nombreuses vues perspectives. Certaines sont des vues générales traditionnelles, avec un point de vue légèrement surélevé, idéalisé ${ }^{34}$. D'autres sont plus proches de l'expérience réelle, presque instantanées tout en demeurant unifiées ${ }^{35}$. Et, finalement, certaines sont plus fragmentées bien que prises du point de vue de l'observateur : point de vue oblique, morcellement des éléments architecturaux, clair-obscur accentué, etc. ${ }^{36}$ [fig. 6].

L'ensemble de ces images témoigne d'un changement d'optique, devenu plus spatial et plus atmosphérique. L'appareil photographique dissout l'unité «classique » en faveur d'une nouvelle forme de compréhension, où le mouvement dans l'espace prime sur la compréhension statique de l'objet.

Ce qui rend l'ouvrage de Narjoux encore plus intéressant que la Gazette des architectes, c'est qu'un si grand nombre de vues soit rassemblé dans un même ouvrage et, surtout, que cet ouvrage soit une publication officielle. Les quatre tomes de Narjoux peuvent donc servir pour marquer un tournant dans l'établissement d'un nouveau régime visuel après 1880. Rappelons qu'ils paraissent entre 1881 et 1883, c'est-à-dire au début de cette décennie décisive dans laquelle la photographie s'intègre de façon définitive à l'édition architecturale.

Sylvain BELLENGER et Françoise HAMON (dir.), Félix Duban, 1798-1870: Les couleurs de l'architecte, Paris, Gallimard/Electa, 1996. 


\section{BIBLIOGRAPHIE}

Béatrice BOUVIER, L'édition d'architecture à Paris au XIX siècle : les maisons Bance et Morel et la presse architecturale, Genève, Librairie Droz, 2004.

Martin BRESSANI et Peter SEALY, «The Opéra Disseminated: Charles Garnier's Le Nouvel Opéra de Paris (1875-1881) », dans Stephen BANN (dir.), Art and the Early Photographic Album, Washington, National Gallery of Art, 2011, p. 196-219.

Paul Dufournet, Claudine de VAULCHIER et Gilbert DumAS (dir.), Catalogue des collections, vol. 1, 1750-1900 : Dessins, photographes, jetons et médailles, effigies d'architectes, Paris, Académie d'Architecture, 1988.

Charles Pierre GOURLIER, Jean Marie Dieudonné BIET et al., Choix d'édifices publics projetés et construits en France depuis le commencement du XIX ${ }^{e}$ siècle, Paris, Louis Colas, 1825-1850.

Charles GARNIER, Le Nouvel Opéra de Paris, Paris, Ducher et Cie., 1880.

Marie-Noëlle LEROY, « Le monument photographique des frères Bisson », Études photographiques, $\mathrm{n}^{\circ} 2,1997$.

Neil LEVINE, Architectural Reasoning in the Age of Positivism: The Néo-Grec Idea of Henri Labrouste's Bibliothèque Sainte-Geneviève, thèse doctorale, Yale University, 1975.

Neil LEVINE, « The Template of Photography in Nineteenth-century Architectural Representation ", Journal of the Society of Architectural Historians 71, $\mathrm{n}^{\circ}$ 3, 2012.

Hélène LIPSTADT, « The Building and the Book in César Daly's Revue générale d'architecture » dans Beatriz Colomina (dir.), Architectureproduction, New York, Princeton Architectural Press, 1988, p. 24-55.

Florent MIANE, « Du document d'étude à l'image illustrative. L'usage de la photographie par des architectes ", Architectes et photographes au XIX siècle, Paris, INHA, 2016.

Félix NARJOUX, Paris, monuments élevés par la ville, 1850-1880, Paris, Vve. A. Morel et Cie., 1880-1883.

Marc SABOYA, Presse et Architecture au XIX siècle : César Daly et la Revue générale de l'architecture et des travaux publics, Paris, Picard, 1991.

Peter SEALY, «After a Photograph, Before Photography [Takes Command] », The Journal of Architecture, à paraître, 2016.

Gazette des architectes et du bâtiment, vol. 1, 1863 ; vol. 5, 1867 ; vol. 6, 1868-1869 ; vol. 7, 1869-1871.

Revue générale de l'architecture et des travaux publics, vol. 6, 1845-1846 ; vol. 16, 1858 ; vol. 19, 1861 ; vol. 29,1872 ; vol. 30,1873 ; vol. 31,1874 ; vol. $35,1878$.

\section{NOTES}

1. Charles Pierre Gourlier, Jean Marie Dieudonné Biet et al., Choix d'édifices publics projetés et construits en France depuis le commencement du XIX ${ }^{\mathrm{e}}$ siècle, Paris, Louis Colas, 1825-1850.

2. « Rapport adressé à Monsieur le Ministre de l'Intérieur, par l'Académie Royale des Beaux-Arts sur le premier volume du Choix d'édifices publics », dans Gourlier et Biet 1850, vol. 1, n. p. 
3. Félix Narjoux, Paris, monuments élevés par la ville, 1850-1880, Paris, Vve. A. Morel et Cie., 1880-1883.

4. Par exemple, voir la perspective d'Emmanuel Brune pour l'« escalier principal d'un palais d'un souverain » pour le Grand Prix de 1863 ; la perspective de Charles Garnier de l'escalier principal du nouvel opéra de Paris, "Grand escalier d'honneur, vue en perspective », dans Le Nouvel Opéra de Paris, vol. II, Paris, Ducher et Cie., 1880, pl. 8 et la perspective d'Eugène-Emmanuel Viollet-leDuc pour sa soumission au concours de ce même opéra en 1860-61.

5. Martin Bressani et Peter Sealy, «The Opéra Disseminated: Charles Garnier's Le Nouvel Opéra de Paris (1875-1881)», dans Stephen Bann (dir.), Art and the Early Photographic Album, Washington, National Gallery of Art, 2011, p. 196-219. Cette publication suit une présentation faite à la Center for Advanced Studies in the Visual Arts à Washington, D.C. en 2007.

6. Sur l'introduction du dessin en perspective dans la Revue générale, voir Hélène Lipstadt, «The Building and the Book in César Daly's Revue générale d'architecture » dans Beatriz Colomina (dir.), Architectureproduction, New York, Princeton Architectural Press, 1988, p. 24-55 ; Marc Saboya, Presse et Architecture au XIX ${ }^{\mathrm{e}}$ siècle : César Daly et la Revue générale de l'architecture et des travaux publics, Paris, Picard, 1991, p. 233-241 ; Bressani et Sealy, dans Bann 2011, p. 219, n. 64 ; et Neil Levine, "The Template of Photography in Nineteenth-century Architectural Representation ", Journal of the Society of Architectural Historians 71, $\mathrm{n}^{\circ}$ 3, 2012, p. 315-318.

7. Levine 2012, p.306-331. La photographie fait partie des collections de l'Académie d'Architecture et a été publiée dans Paul Dufournet, Claudine de Vaulchier et Gilbert Dumas (dir.), Catalogue des collections, vol. 1, 1750-1900: Dessins, photographes, jetons et médailles, effigies d'architectes, Paris, Académie d'Architecture, 1988.

8. Levine 2012 , p. 307. Le premier exemple connu d'une gravure faite d'après une photographie dans un périodique architectural date de 1845, quand la revue britannique The Builder a publié une vue du mausolée de la dynastie Orléans à Dreux (Normandie) par l'architecte Pierre-Bernard Lefranc. Le texte qui accompagne cette image confirme qu'elle a été produite d'après un daguerréotype, mais les circonstances dans lesquelles cette photographie a été prise ne sont pas connues. Voir Peter Sealy, «After a Photograph, Before Photography [Takes Command] », The Journal of Architecture (à paraître, 2016).

9. César Daly, «École Royale des Beaux-Arts de Paris. Ouverture des cours de M. Blouet, professeur de théorie et d'architecture, et de M. Constant Dufeux, professeur de perspective ", Revue générale de l'architecture et des travaux publics, vol.6, 1845-1846, col.522. Cité dans Levine 2012, n. 9, p. 317 et n. 37, p. 330.

10. Par exemple, le «Plan et vues extérieure et intérieure de l'École de Beaux-Arts » dans l'Album du duc d'Orléans offert au prince-héritier de Prusse (1837), qui fait partie des collections des Staatliche Museen zu Berlin. Cette aquarelle est reproduite dans Sylvain Bellenger et Françoise Hamon (dir.), Félix Duban, 1798-1870 : Les couleurs de l'architecte, Paris, Gallimard/Electa, 1996, fig. 33.

11. Marie-Noëlle Leroy, "Le monument photographique des frères Bisson ", Études photographiques, $\mathrm{n}^{\circ} 2,1997$, p. 92.

12. Ibid., p. 86.

13. "Esquisse de vue en perspective », bibliothèque Sainte-Geneviève, Ms. 4273(99) / recto. La bibliothèque Sainte-Geneviève date ce croquis d'«avant 1842 " tandis que Levine donne 1839 comme date approximative. Voir Neil Levine, Architectural Reasoning in the Age of Positivism: The Néo-Grec Idea of Henri Labrouste's Bibliothèque Sainte-Geneviève, thèse doctorale, Yale University, 1975, vol. IV, fig. 170.

14. Monographie de la maison gréco-romaine construite pour sa majesté impérial Mgr le prince Napoléon par M. Alfred Normand et photographié par J. Laplanche, Paris, J. Laplanche et Cie, s. d. 
15. Gustave Boulanger, La répétition du joueur de flûte et de la femme de Diomède chez le prince Napoléon dans l'atrium de sa maison pompéienne, 1860, Versailles, musée national des châteaux de Versailles et de Trianon.

16. Par exemple, la Vue du phare de Cordouan à l'embouchure de Gironde porte l'indication «D'après une photographie de M. Terpereau ». Voir la Revue générale de l'architecture et des travaux publics, vol. 35, 1878, pl.47. Sur Terpereau, voir l'essai de Florent Miane dans cette publication, «Du document d'étude à l'image illustrative. L'usage de la photographie par des architectes».

17. Selon Levine, les planches 56 et 58 de la Revue générale de l'architecture et des travaux publics, vol. 30, 1873, sont dessinées à partir d'une photographie. Levine 2012, n. 9, col. 331, n. 67. 18. Trois vues perspectives, Portique de la façade principale, Pavillon sur le jardin et Vue de la galerie, furent publiées de l'hôtel de M. Fould dans la Revue générale de l'architecture et des travaux publics, vol. 16, 1858, pl. 8, 9 et 10. La Revue générale a publié neuf vues en perspective des projets du concours pour le grand Opéra de Paris. Voir Revue générale de l'architecture et des travaux publics, vol. 19, 1861, pl. 27, 29, 30, 31, 35 et 40 .

19. Parmi de nombreux exemples, citons un pour chaque catégorie: Hôtel privé, boulevard Exelmans, à Auteil. Vue perspective, Revue générale de l'architecture et des travaux publics, vol. 30, 1873, p. 59 (pittoresque); Monument élevé à la mémoire du maréchal Moncey, Place de Clichy, à Paris, ibid., vol. 29, 1872, pl. 35 (urbaine) ; Tourelle, rue Hautefeuille, à Paris (xvi siècle), ibid., vol. 30, 1873, pl. 13 (historique).

20. Hôtel du journal le Figaro, rue Drouot, à Paris. Façade principale en perspective, Revue générale de l'architecture et des travaux publics, vol. 31, 1874, pl. 46.

21. Salle du Public, ibid., pl. 49.

22. Ministère de l'Agriculture, du Commerce et des Travaux publics, à Paris. Vue intérieure en perspective, Revue générale de l'architecture et des travaux publics, vol. 29, 1872, pl. 47.

23. Vue intérieure en perspective, ibid., pl. 48.

24. Sur la Gazette des architectes et du bâtiment, voir Béatrice Bouvier, L'édition d'architecture à Paris au XIX ${ }^{\mathrm{e}}$ siècle : les maisons Bance et Morel et la presse architecturale, Genève, Librairie Droz, 2004.

25. "Article préliminaire », Gazette des architectes et du bâtiment, vol. 1, 1863, p. 3. Cité dans Bouvier 2004, n. 29, p. 103.

26. David Sutter, « Nouveau traité de perspective », Gazette des architectes et du bâtiment, vol. 5, 1867, p. 213.

27. Bouvier 2004, n. 29, p. 103.

28. Chaire dans l'église abbaye des moines bénédictins à Fulda (province de Hesse), en Prusse, Gazette des architectes et du bâtiment, vol. 7, 1869-1871, fig. 53. Un article de Choisy intitulé «L'Économie dans la construction romaine » a paru dans ce même volume.

29. Propriété de M. W..., à Suresnes (Seine). M. Darcy, architecte, Gazette des architectes et du bâtiment, vol. 6, 1868-1869, p. 169-174, 199-200 et 249-251 ; vol. 7, 1869-1871, p. 24-26, 54-57, $127-132,156-160$ et $172-175$.

30. Vue perspective prise du jardin, Gazette des architectes et du bâtiment, vol. 6, 1868-1869, fig. 97.

31. Vue perspective des bâtiments d'habitation vers l'entrée, Salle à manger (Face vers le jardin d'hiver), Salle à manger (Tambour de l'escalier de service), Bibliothèque, Perspective, Bibliothèque, Perspective, Salon de musique, Gazette des architectes et du bâtiment, vol.6, 1868-1869, fig. 117, vol. 7, 1869-1871, fig. 43, 46, 90-91 et 116.

32. Anatole de Baudot, «Propriété de M. W..., à Suresnes (Seine). M. Darcy, architecte. Notice descriptive ", Gazette des architectes et du bâtiment, vol. 6, 1868-1869, p. 169.

33. Villa Honoré, à Trouville-sur-mer. M. Devrez, architecte. Perspective générale, Perspective de porche, Perspective de la terrasse, Perspective vers l'entrée, Vue perspective (Façade nord.) et 
Vue perpsective (Façade principale.), Gazette des architectes et du bâtiment, vol. 7, 1869-1871, fig. 105, 201, 202, 204, 209 et 211.

34. Par exemple, Palais de justice, Élévation sur le boulevard du Palais, pl. III, vol. I ; Tribunal de

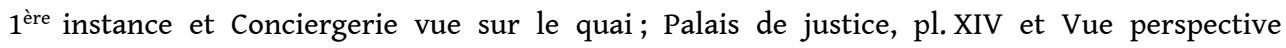
extérieure ; Marché des martyrs, pl. II, vol. II, Narjoux 1880-1883.

35. Par exemple, Fontaine de l'observatoire, Narjoux 1880-1883, vol. IV.

36. Par exemple, Vue de la salle des mariages; Mairie du IVe arr., fig. 1, Escalier d'honneur; Mairie du XIII ${ }^{e}$ arr., fig. 1, Vestibule ; Mairie du XX ${ }^{\mathrm{e}}$ arr., fig. 1, Escalier d'honneur ; Mairie du XX arr., fig. 2 et Police correctionnelle - vestibule; Palais de justice, pl. XVIII vol. I; Vue d'un des grands escaliers; Groupe scolaire, rue Curial, pl. III et Vue de la galerie couverte; Groupe scolaire, rue Curial, pl. III, vol. II ; Vue perspective intérieure ; Église Saint-Joseph, pl. III, Vue du vestibule ; Théâtre du Châtelet, fig. 1, Loge ouverte du premier étage ; Théâtre du Châtelet, fig. 2 et Vestibule ; Théatre-Lyrique, fig. 1, vol. III ; Vue perspective intérieure (côté de l'entrée) ; HotelDieu, pl. III, vol. IV, Narjoux 1880-1883.

\section{AUTEURS}

\section{MARTIN BRESSANI}

McGill University School of Architecture

\section{PETER SEALY}

Harvard University Graduate School of Arts and Sciences 


\title{
Photographes et architectes à Rome au XIX ${ }^{\mathrm{e}}$ siècle
}

\author{
Collaboration et culture partagée, le cas des légendes Chauffourier \\ Jean-Philippe Garric
}

1 Note au lecteur : À cause de la nature complexe du corpus concerné et du caractère encore récent des études sur la photographie romaine de la deuxième moitié du XIX siècle, certaines attributions actuelles sont susceptibles d'évoluer. Dans le cadre de cet article, nous avons toutefois préféré nous en tenir à celles aujourd'hui admises dans les publications récentes.

2 Autour des ruines, des édifices et des paysages de Rome, objets d'intérêt et d'études communs aux architectes et aux photographes, les indices de leurs échanges montrent comment les premiers ont sollicité les seconds pour compléter leurs documentations. Mais ils permettent aussi d'inscrire ces relations dans une tradition artistique et culturelle partagée, marquée par l'héritage des architectes peintres des générations précédentes.

3 Tommaso Cuccioni, qui jouissait déjà d'une grande notoriété comme graveur, marchand et éditeur de vues gravées de Rome, se lança dans la photographie dès le milieu des années 1850, dans un partenariat, dont on ignore les termes exacts, avec le plus important photographe romain de l'époque, Giacomo Caneva. Privilégiant la production d'épreuves de grandes et de très grandes dimensions, il publia alors deux séries, l'une de format $22 \times 32 \mathrm{~cm}$, l'autre deux fois plus grande, soit $33 \times 46 \mathrm{~cm}$, à laquelle appartient une vue verticale très classique du temple de la Sibylle à Tivoli, montrant trois personnages posant et regardant l'opérateur [fig. 1]. Ils sont trop loin pour qu'on distingue les traits de leurs visages, mais leurs tenues caractéristiques permettent cependant d'écarter l'hypothèse de simples badauds et de faire quelques conjectures. 


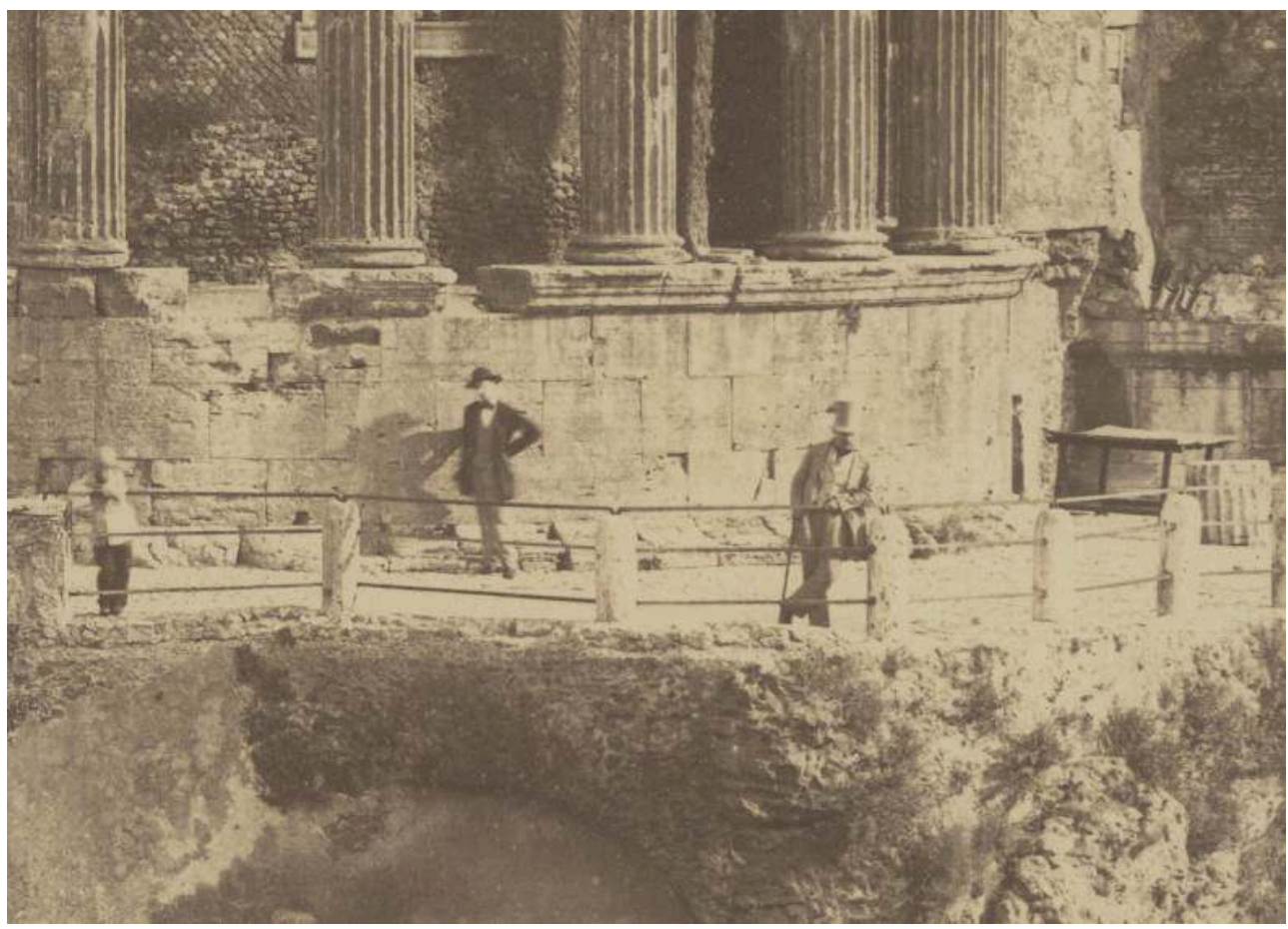

Fig. 1 : Tommaso Cuccioni, Vue verticale du temple de la Sibylle à Tivoli (détail), tirage albuminé d'après négatif verre, $46 \times 33 \mathrm{~cm}$, Paris, collection particulière.

4 Les deux premiers sont accoudés à la balustrade. Celui de gauche, dont la taille et l'apparence sont celles d'un enfant, est habillé d'une veste blanche ou d'une blouse. C'est sans doute un apprenti ou un jeune assistant du photographe, comme on en voit assez souvent sur les clichés de cette époque. Le deuxième, à droite, négligemment accoudé au garde-corps, le regard plongé vers le bas du ravin, porte un vêtement d'une élégance presque excessive, dans un contexte aussi pittoresque et surtout peu urbain : un costume clair trois pièces, un immense chapeau à cylindre assorti et une canne dans la main droite. On dirait qu'il a des moustaches. L'homme, qui apparaît sur d'autres photos contemporaines vendues par Cuccioni, est probablement le marchand luimême. L'autre se tient debout au centre, légèrement en retrait contre le soubassement du temple, équidistant des deux premiers. Chapeau mou noir à grands bords, longue veste en toile noire, nœud papillon, pantalon et gilet gris : il a toute l'apparence d'un architecte français. Alors, architecte et photographe? Peut-être. Mais travaillaient-ils de concert, ou était-ce le hasard qui les réunissait ce jour-là au pied du monument ? Audelà de telles conjectures, si une telle rencontre était confirmée, elle ferait figure d'exception dans la production photographique romaine du $\mathrm{XIX}^{\mathrm{e}}$ siècle. Parmi les milliers de vues qui nous sont parvenues, quelques-unes, peu nombreuses, montrent des archéologues à l'ouvrage, en particulier dans les albums Parker, mais les architectes sont rarissimes, voire entièrement absents. Il était pourtant fréquent que le photographe, ou ceux qui l'accompagnaient, profitent de l'occasion pour passer euxmêmes, comme ici, de l'autre côté de l'objectif.

5 Par ailleurs, architectes et photographes travaillaient souvent à cette époque sur les mêmes sites: des édifices qui constituaient de longue date pour les premiers leur principale raison d'entreprendre le voyage d'Italie et qui étaient pour les seconds des modèles adaptés à une époque de temps de pause encore trop prolongés pour saisir le mouvement. Pourtant, en dépit de cette relative lenteur, les performances de la 
photographie, disqualifiant le patient travail du dessin, dont elle n'avait pas la valeur heuristique, suscitaient une inquiétude, qui transparaît parfois sous la plume d'architectes contemporains, comme lorsqu'Aymar Verdier note en avertissement de son ouvrage sur l'architecture domestique, consacré en partie aux maisons d'Italie :

6 «[...] notre travail sera peut-être un des derniers de cette nature permis encore par la science dévouée du dessin; qu'en effet les procédés nouveaux de la photographie tendent à envahir chaque jour les prérogatives du crayon. Qu'il est vrai pourtant de dire que les lois de la perspective ne peuvent changer et qu'il sera toujours impossible à cette invention récente de reproduire la plupart des monuments que nous avons recueillis de préférence. Les auteurs en général s'applaudissent avec raison d'être les premiers entrés dans une voie nouvelle. Quant à nous, nous nous estimerions plus heureux encore si le sort nous destinait à être les derniers à suivre la vieille voie de l'estampe, pour nous servir de la caractéristique expression de nos devanciers : car malgré l'exactitude mathématique qui est la perfection des produits de la lumière, les œuvres de la main de l'homme auront toujours une expression de vie, un trait d'intelligence qui manquera constamment aux premiers ${ }^{1}$.»

7 Au-delà de cette opposition formulée dans des termes convenus, mais qui ne sont pas sans évoquer ceux qui circulent souvent aujourd'hui à propos des révolutions induites par le développement des médias numériques, la concurrence de la nouvelle technique et les inquiétudes l'accompagnant n'étaient pas de nature à interdire toute collaboration. Du reste, la mécanisation du dessin avait précédé de plusieurs décennies l'invention du nouveau procédé. S'il n'était pas encore possible, dans la première moitié du XIX ${ }^{e}$ siècle, de fixer les images directement sur le papier recouvert d'une préparation sensible, par le seul effet de la lumière, on pouvait néanmoins déjà en voir la projection, qu'il était facile de copier manuellement, notamment depuis que les chambres claires, brevetées en 1806 par le Britannique William Hyde Wollaston (1766-1828), s'étaient banalisées. Ce passage d'une lettre adressée depuis Rome par Léon Vaudoyer à son père en 1827 en donne une bonne illustration :

8 «Si maintenant je veux parler des objets nécessaires pour travailler, vous conviendrez avec moi qu'il me faut avoir un plomb, un mètre à niveau pour mesurer les saillies puis enfin la chose de première nécessité ici et que vous considérerez, je pense, comme une chose à part ; c'est une chambre claire : avec le pied et la planche cela coûte 60 francs. Elles sont bien meilleures ici qu'à Paris ${ }^{2}$.»

9 Certes, en dépit des parentés entre chambre claire et chambre noire, la première n'était qu'un outil au service des vedutistes insuffisamment familiers des lois de la perspective, tandis que la seconde permettait à tout un chacun de le concurrencer. Toutefois, l'exemple d'Alfred-Nicolas Normand ${ }^{3}$, Grand Prix de Rome qui compte parmi les pionniers de la photographie romaine, montre que les architectes ne furent pas les derniers à tester le nouveau procédé. On connaît, par ailleurs, les ensembles d'épreuves rapportées par plusieurs d'entre eux - qu'il s'agisse d'une documentation professionnelle, de collections de vues pittoresques, ou un peu des deux à la fois. La collection la plus emblématique, à cause de la personnalité de son auteur, est sans doute celle de Charles Garnier. On peut penser que s'il choisit d'acquérir auprès de James Anderson une vue de la piazza Navona immergée par le lac estival, c'était moins pour son caractère documentaire que pour la singularité de la pratique urbaine représentée et pour l'atmosphère de l'image, qui tient tout autant au sujet qu'à l'éclairage un peu irréel, caractéristique des négatifs à l'albumine ${ }^{4}$. 

voyageurs cultivés présents et actifs à Rome à la même époque, qui collectionnaient eux aussi les épreuves photographiques et se lançaient parfois dans la prise de vues. Il est d'ailleurs difficile de reconnaître dans la production d'un Normand un regard proprement "architectural», qui distinguerait ses images de la production contemporaine des autres membres du cercle du Caffè Greco.

Dans les publications architecturales de l'époque, l'expansion de la photographie ne donne pas lieu à des mutations rapides et importantes. Les essais du directeur de la Revue générale de l'architecture César Daly pour publier en 1856 deux planches photographiques, dont l'une comporte une épreuve originale sur papier salé et l'autre une reproduction en phototypie ${ }^{5}$, sont bien connus. Mais ces tentatives furent sans suite directe et, dans le dernier volume du même périodique, aussi tard qu'en 1888, on continuait de reproduire les photographies par le biais de la gravure. C'est notamment le cas d'une vue du portail du palais Zucchari, occupant la planche $1 \mathrm{du}$ volume 45 [fig. 2].

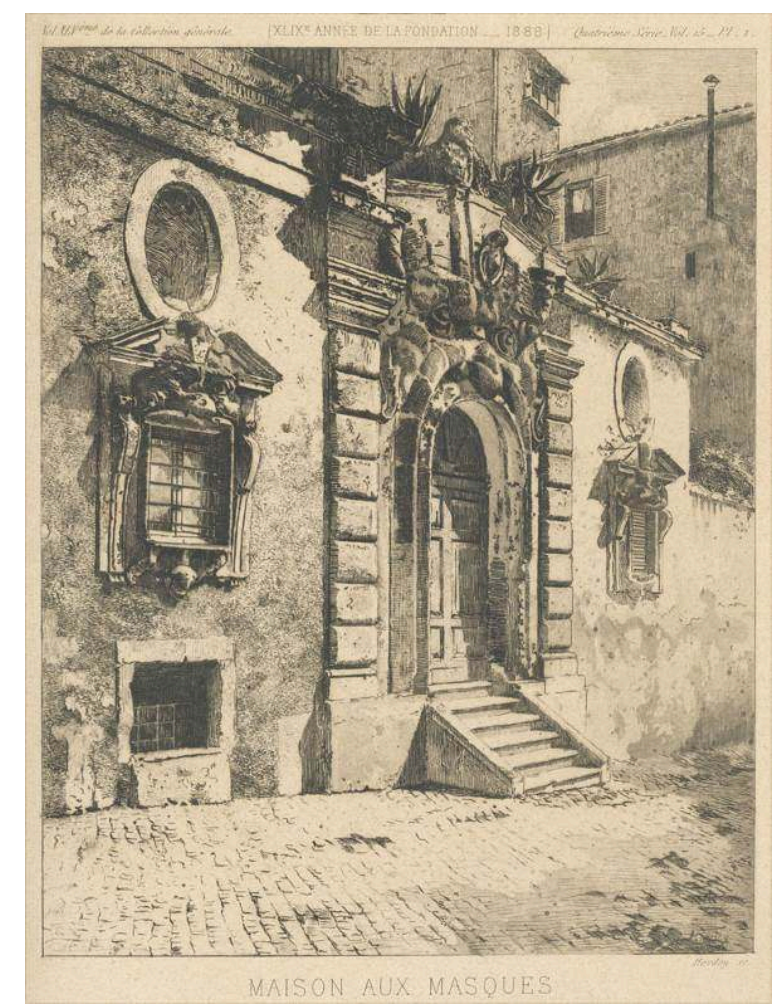

Fig. 2 : Herdey (graveur), Vue du portail du palais Zucchari à Rome, dans César Daly, Revue générale de l'architecture, vol. 45, 1888, pl. 1.

Cette vue est en tout point singulière. Par son objet d'abord, assez loin du rationalisme des premières années de la revue, qui ne regardait pas alors vers l'Italie baroque, par sa technique ensuite, conjuguant l'eau-forte et l'aquatinte, et par son auteur. À ce propos, Daly précise que : «[...] c'est d'après une photographie que la pl. 1 a été exécutée. Je la tenais, ajoute-t-il, de l'obligeance de notre éminent confrère $M$. Pascal, alors encore élève de Rome [...]». Plus loin, il note également que la signature de Herdey est le pseudonyme d'un «habile amateur» qu'il aimerait "voir plus souvent au bas des Planches de la Revue ${ }^{6} »$. On connaît donc le nom de l'architecte ayant fourni l'image et le pseudonyme du graveur, mais on ignore l'identité du photographe. 
13 Jean-Louis Pascal avait été pensionnaire à Rome de 1867 à 1870. C'est donc dans ces années-là qu'il transmit l'image à Daly ; ce dernier ayant remarqué l'étonnant édifice, comme chaque touriste curieux peut le faire, « un jour en allant à la Villa ». Par chance, une autre épreuve ayant récemment rejoint les collections photographiques du musée d'Orsay permet de l'attribuer. Elle provient du fonds Edmond Lebel, peintre, qui, sans avoir été Prix de Rome, séjourna à Rome à partir de $1861^{7}$, et elle porte le cachet d'Adriano De Bonis. Celui-ci, photographe discret dont le nom n'apparaît pas dans les énumérations des annuaires de l'époque, marquait ses épreuves d'un tampon humide, puis plus tard d'un petit cachet, aussi sibyllins l'un que l'autre ${ }^{8}$, mais qui témoignent certainement d'une conscience de sa propre valeur artistique.

14 Les autres éléments connus de son activité permettent de le situer comme un photographe s'adressant à un public plus spécialisé que la masse des visiteurs auxquels s'adressaient les principales agences romaines ayant pignon sur rue, voire comme un prestataire travaillant à la demande pour d'autres professionnels ${ }^{9}$. Loin de se limiter à la représentation des sites et des monuments les plus célèbres, il s'écarte souvent des sentiers battus, pour reprendre le titre du premier ouvrage lui ayant consacré une place importante ${ }^{10}$. Un autre ensemble de ses photographies appartient au fonds Justin Sanson $^{11}$, un sculpteur présent à Rome de 1862 à 1866, qui complète ainsi avec l'architecte Pascal et le peintre Lebel une première liste de clients artistes. On sait par ailleurs que certaines de ses épreuves étaient vendues à Paris revêtues du cachet d'Achille Quinet [fig. 3], tandis que Francis Wey fit appel à lui, ou du moins se servit de ses photographies, pour faire réaliser les gravures de son volumineux best-seller sur Rome $^{12}$. Dans ce cas non plus, son nom n'apparaît pas, contrairement à ceux des dessinateurs qui assurèrent la retranscription de ses vues dans une technique plus adaptée à l'imprimerie contemporaine. Enfin, et c'est par ce biais qu'on a pu connaître son patronyme, il fut l'un des photographes actifs pour John Henry Parker, en lien direct avec Carlo Baldassare Simelli sur lequel nous reviendrons ${ }^{13}$. 


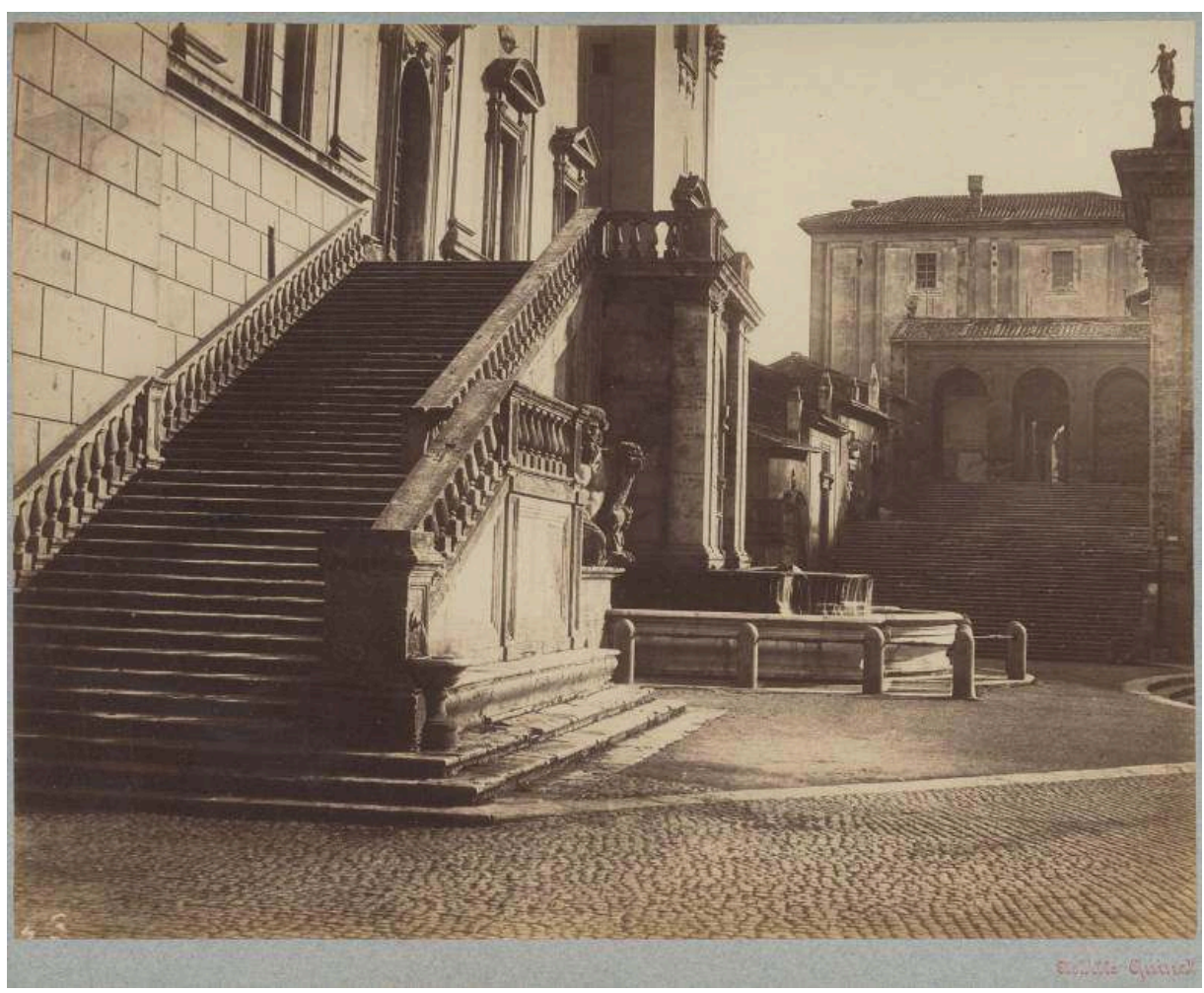

Fig. 3 : Adriano De Bonis, Vue de la place du capitole à Rome, tirage albuminé d'après négatif verre, 19,5 × 24,8 cm, monté sur un carton bleuté portant le cachet rouge d'Achille Quinet, Paris, collection particulière.

15 L'attribution à De Bonis d'une vue du jardin de la villa Médicis, appartenant au fonds Duban de l'INHA et reproduite ici même par Hélène Bocard [Hélène Bocard, «Le fonds photographique des architectes Ballu (INHA)», fig. 2], s'est également faite par recoupement avec une autre épreuve du même négatif qui porte son cachet ${ }^{14}$. Théodore Ballu avait séjourné à Rome dans les années 1840, trop tôt pour en rapporter des photographies, et, si l'on ne connaît pas la date d'achat de ce tirage, il présente une légende manuscrite ajoutée dans le négatif, correspondant à l'exploitation tardive de la plaque de verre par Gustave Chauffourier, à partir des années $1870^{15}$.

16 Or c'est justement au sein de cet ensemble d'épreuves, qui portent le même type de « légendes Chauffourier » manuscrites, que figurent de façon récurrente des travaux se distinguant des habituelles vues pittoresques ou des représentations les plus convenues des principaux monuments de Rome, liés à une approche plus spécifique et détaillée intéressant l'architecture ou l'archéologie. Ces légendes, si elles s'inscrivent toutes dans l'image de façon libre, voire négligée, employant un même type d'écriture et se composant toutes d'un numéro suivi du nom de la ville en capitale, puis du nom du sujet en minuscules, ne recouvrent pas pour autant un corpus homogène. Étudiées plus attentivement, elles révèlent au contraire son caractère composite. Si la plupart sont sombres sur fond plus clair, quelques-unes sont, au contraire, claires sur fond sombre et l'on peut distinguer, entre les légendes sombres, celles qui résultent d'une gravure opérée à la plume ou à la pointe sèche dans la couche sensible de la plaque de verre afin de la dégarnir, celles qui furent ajoutées manuellement directement sur le tirage parfois sur papier salé - et celles, enfin, qui furent contretypées avec l'image [fig. 4]. Cette variété technique dénote la constitution d'un catalogue à partir d'éléments de différentes origines, produits à des époques variées : des plaques provenant sans doute 
de l'activité de plusieurs photographes et des lots d'épreuves sans le négatif correspondant, qu'il fallait contretyper pour continuer à les maintenir au catalogue. Les deux auteurs de cet ensemble identifiés jusqu'à présent sont De Bonis et Simelli.

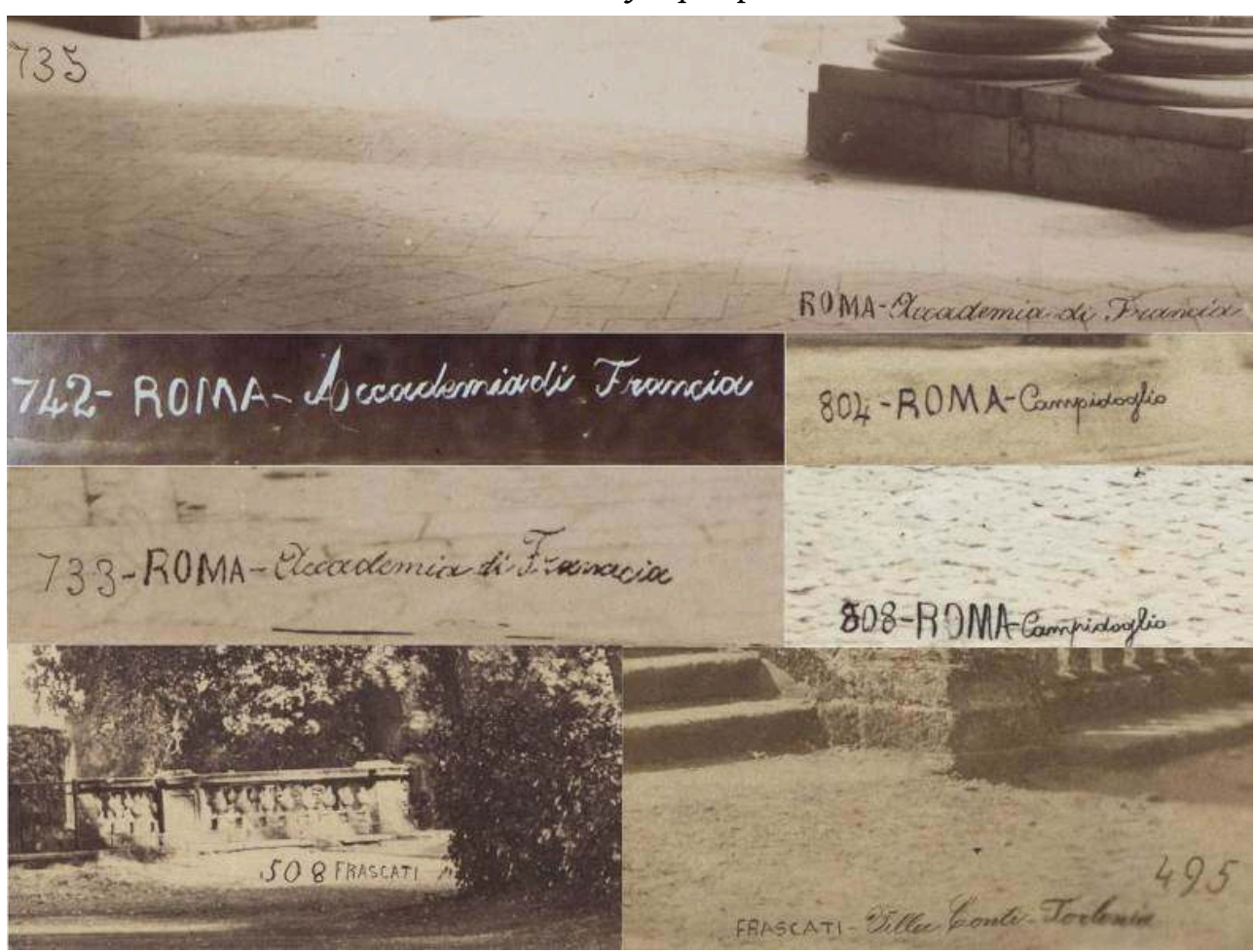

Fig. 4 : Comparaison de différentes signatures de Chauffourier en clair ou en sombre dans le négatif, qui découlent de différentes techniques : gravure dans la couche sensible du négatif, écriture en sombre sur le négatif ou contretype d'une épreuve légendée.

Si l'on ne dispose que d'indices ponctuels, comme ceux que nous venons d'évoquer, sur les commandes qui pourraient être à l'origine d'une partie de ces photographies, deux pistes permettent cependant d'explorer plus avant les relations entre ces photographes et les architectes. La première concerne l'analyse de leur production, la seconde leurs parcours personnels. Sur le premier point, certaines séries, par leur nature comme par leur développement, montrent un souci de documentation approfondie d'un édifice, qui relève d'une véritable démarche de relevé photographique. C'est le cas, en particulier, du dossier concernant le palais de la Chancellerie. Si l'on y trouve des vues d'ensemble se rattachant à une production plus courante, il contient aussi des détails spécifiques: chapiteau du niveau bas de la cour, détail de la corniche d'une porte donnant sur la cour au piano nobile ou fenêtre d'angle extérieure de l'édifice avec balcon $^{16}$ [fig. 5]. Cette dernière image, contrairement aux deux précédentes dont la composition et l'éclairage sont strictement utilitaires, se distingue d'ailleurs par la façon très ambiguë dont est traité l'élément d'architecture, l'auteur utilisant des masses d'ombre en bas et surtout en bordure gauche de la photographie pour créer un effet dramatique auquel font écho les dégradations physiques du sujet: appareil de l'arc en partie disjoint avec déplacement du claveau central; carreau brisé de la fenêtre; différence de couleur de la pierre de part et d'autre de la fenêtre en partie basse. 


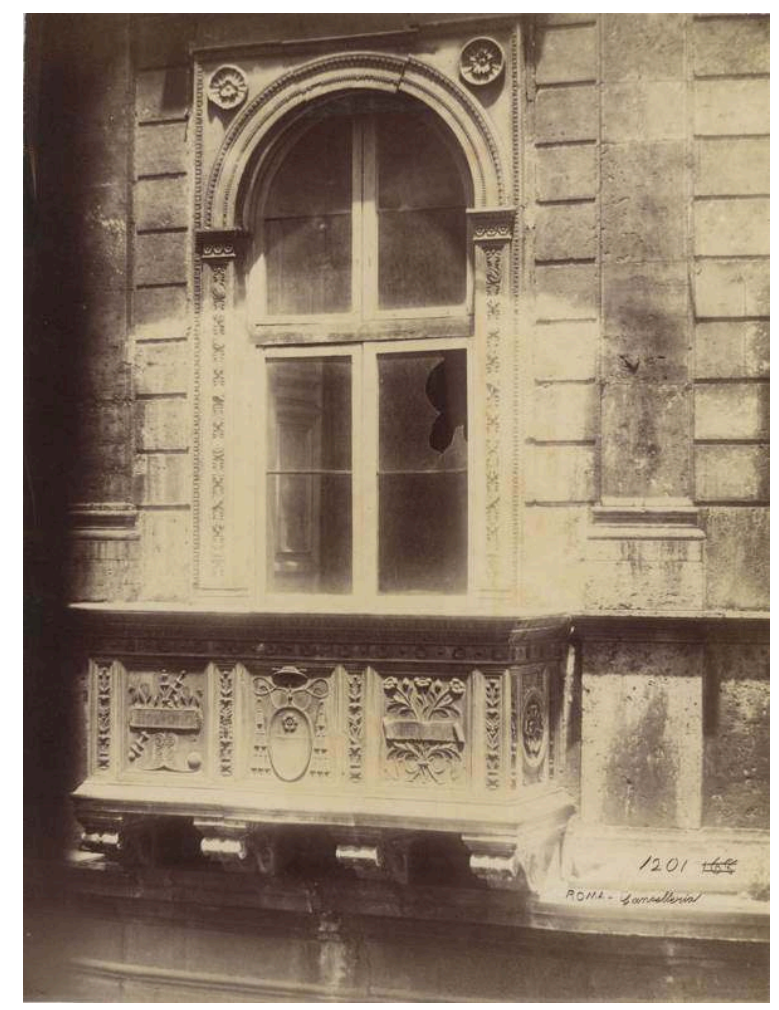

Fig. 5 : Gustave Chauffourier d'après un négatif d'Adriano De Bonis, Vue de la fenêtre d'angle du palais de la Chancellerie à Rome, tirage albuminé d'après négatif verre, $24,6 \times 18,6 \mathrm{~cm}$, Paris, collection particulière.

18 Au-delà du nombre de prises de vue et du caractère détaillé de plusieurs d'entre elles, le reportage sur le palais de la Chancellerie comprend également six photographies exposées de façon strictement frontale, lesquelles, assemblées trois à trois, permettent de reconstituer les élévations complètes de deux travées de la façade extérieure et de trois travées de l'élévation sur cour. Il existe au moins un exemplaire de ces assemblages, monté à l'époque sur carton brun, qui restitue les proportions de l'édifice aussi bien qu'un dessin d'architecture et qui le surpasse par sa précision ${ }^{17}$ [fig. 6]. Bien qu'un tel dossier ne soit peut-être pas tout à fait unique en son genre et en dépit du fait qu'on ne connaît pas l'origine de cette entreprise, son caractère exceptionnel permet d'affirmer qu'il ne relève pas d'une politique systématique de couverture des grands palais romains, pour lesquels l'équivalent n'existe pas, mais plutôt d'une démarche spécifique, sans doute liée à une commande. 

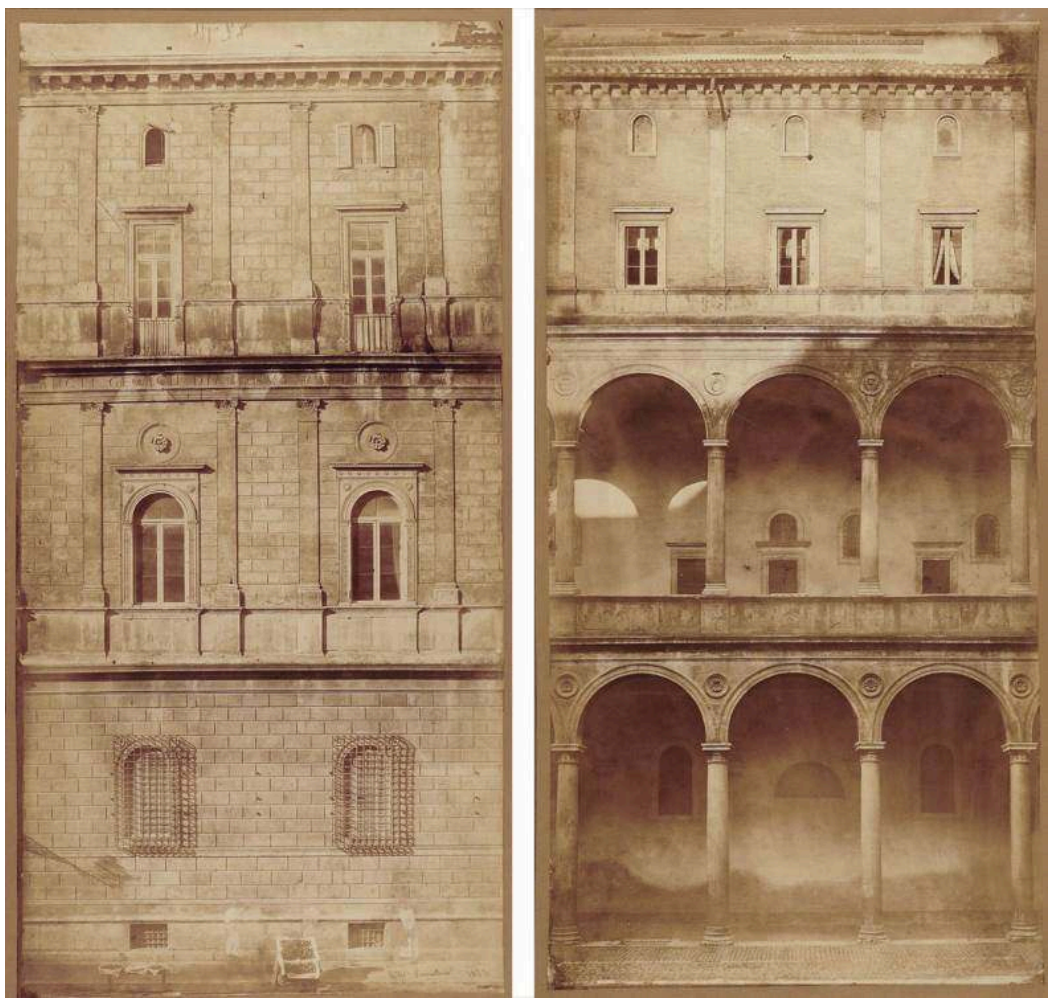

Fig. 6 : Gustave Chauffourier, Palais de la Chancellerie à Rome, montage de trois épreuves albuminées d'après négatif verre sur un même support formant une élévation complète de deux travées de la façade extérieure et montage de trois épreuves albuminées d'après négatif verre sur un même support formant une élévation complète de trois travées de la façade sur cour, New York, collection particulière.

Cet exemple atteste des liens de collaboration fonctionnelle entre architectes et photographes. Pourtant, il serait restrictif de réduire les échanges entre ces deux milieux à la production de documents photographiques spécifiquement destinés à seconder une étude architecturale, car ces relations s'inscrivent dans un contexte beaucoup plus vaste.

On ne connaît pas précisément la nature des formations qu'avaient reçues Simelli ou De Bonis. À propos du premier, Piero Becchetti parle de perspective et de gravure ${ }^{18}$, tandis que le second, avant de s'engager dans le domaine de la photographie, avait déjà fait paraître, en 1851, un petit traité des ordres d'architecture et la traduction en italien du traité de perspective de Jean Pierre Thénot, ce qui le rattachait nettement à la culture architecturale et, au moins de manière indirecte, à la tradition française des architectes peintres ${ }^{19}$. De la première carrière de Simelli, on connaît surtout une suite de quelques gravures à l'aquatinte, dans laquelle se manifeste l'influence du recueil de vues de Rome de Louis-Pierre Baltard ${ }^{20}$. De ces quelques images, nous n'en retiendrons qu'une. Elle montre à quel point cet acteur déterminant de la production photographique romaine pouvait partager avec les architectes des références issues du monde de l'architecture.

Cette perspective, intitulée Roma - Ingresso di una casa alle Quattro Fontane, représente le vestibule d'un édifice fameux dans les milieux artistiques romains : le palais Tomati, Strada Felice, dans lequel Piranèse s'était installé en 1761 [fig. 7]. Mais il est bien difficile de le reconnaître, car en réalité, l'escalier, qui est ici montré profondément plongé dans l'ombre et précédé d'une grande salle entièrement sombre, donnait sur 
une cour et était asymétrique. La configuration plutôt inventive proposée par Simelli trouvait en fait son origine non pas dans un croquis pris in situ à Rome, mais dans la consultation d'un recueil gravé publié à Paris et qui était fondamental pour la culture des architectes français élèves de l'École des beaux-arts : celui que Percier et Fontaine avaient dédié aux palais de Rome en 1798 et dont la planche 31 représentait le Vestibule d'une maison Strada Felice [fig. 8].

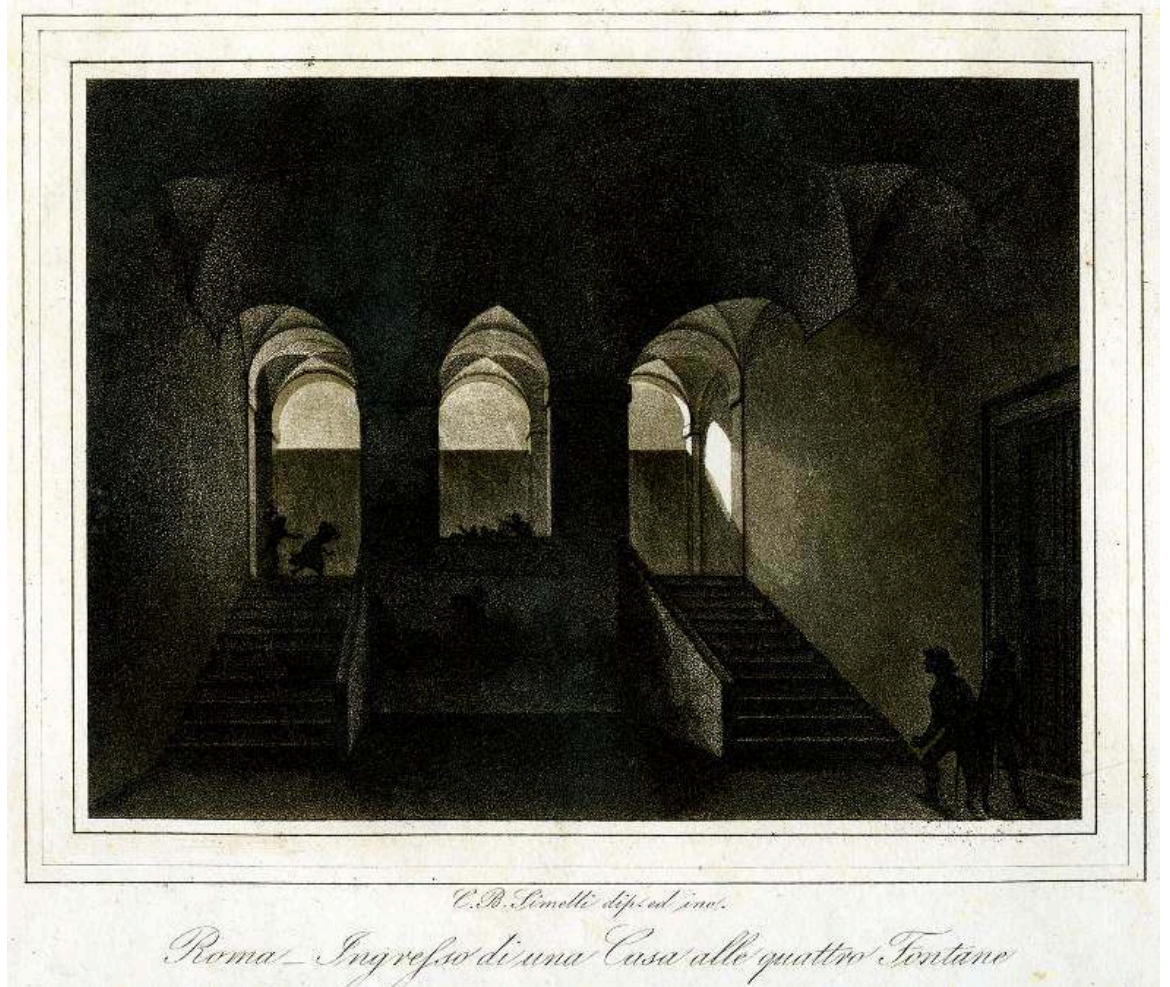

Fig. 7 : Carlo Baldassare Simelli, d'après Percier et Fontaine, Vue du départ de l'escalier du palais Tomati Strada Felice à Rome, aquatinte, Paris, collection particulière. 


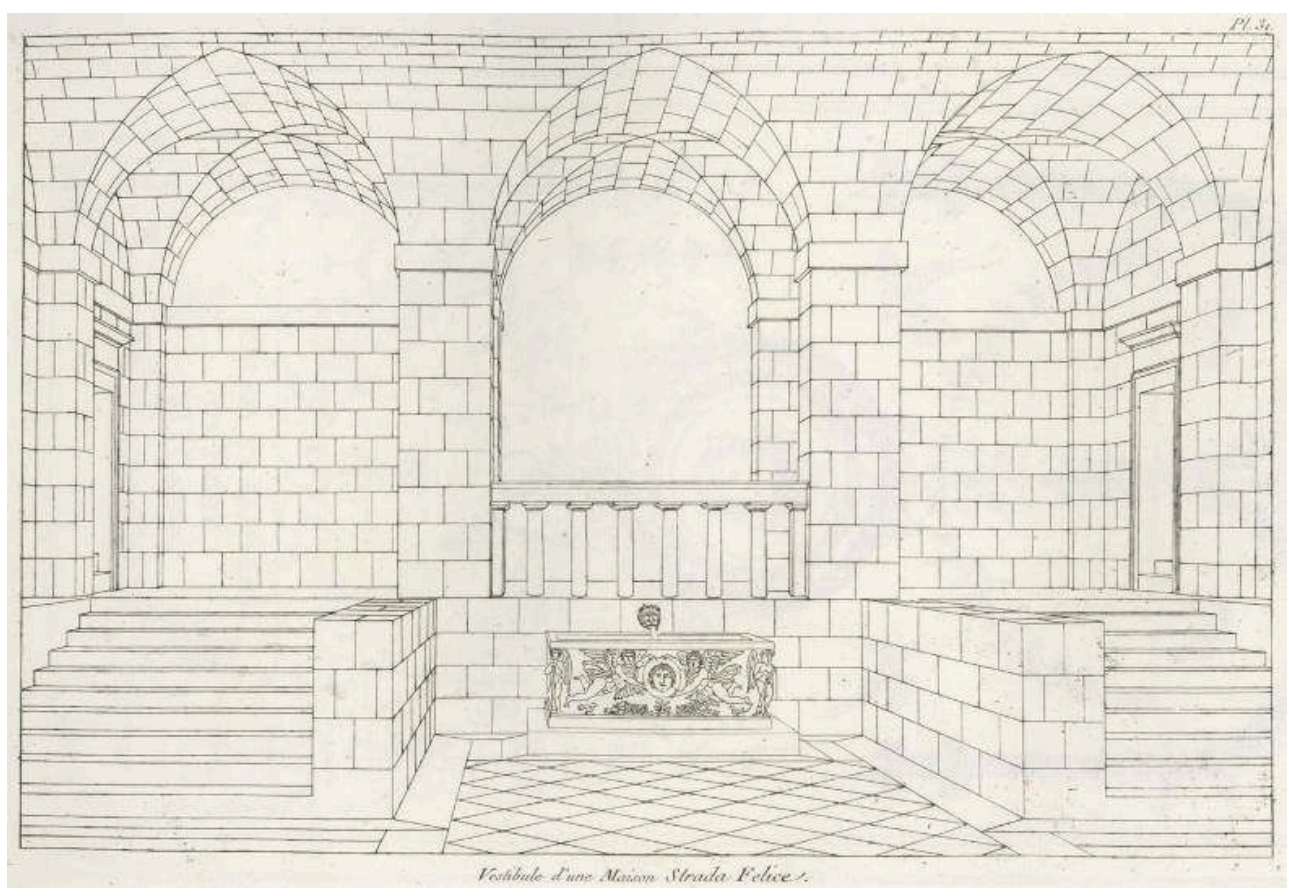

Fig. 8 : Pierre Fontaine, Vue du départ de l'escalier du palais Tomati Strada Felice à Rome, dans Charles Percier et Pierre Fontaine, Palais, maisons et autres édifices modernes dessinés à Rome, Paris, les auteurs, 1798, pl. 31, Paris, collection particulière.

On voit ainsi comment De Bonis et Simelli, s'ils ont alimenté en photographies le milieu des architectes et des artistes français gravitant autour de l'Académie de France à Rome, s'étaient eux-mêmes nourris de références empruntées à la culture de l'École des beaux-arts.

\section{NOTES}

1. Aymar Verdier et François Cattois, "Avertissement ", Architecture civile du Moyen Âge et de la Renaissance, t. 1, 1855, p. III-IV.

2. Lettre de Léon Vaudoyer à son père, Rome, 11 mars 1827, bibliothèque de l'INHA.

3. Voir le catalogue de l'exposition Normand et celui de l'exposition consacrée aux photographes du Cercle du Caffé Greco: Philippe Néagu, Dr Alfred Cayla et André Jammes, A.-N. Normand architecte, photographies de 1851-1852, Paris, Direction des Musées de France, 1978 ; Anne CartierBresson, Anita Margiotta et al., Rome 1850. Le cercle des artistes photographes du Caffé Greco, Milan, Electa, 2003.

4. Voir Bruno Girveau (dir.), Charles Garnier. Un architecte pour un empire, cat. exp., Paris, École nationale supérieure des beaux-arts, Paris, 2010, Paris, Beaux-Arts de Paris, 2010, p. 195.

5. César Daly, Revue générale de l'architecture, vol. 14, 1856, pl. 1 (tirage photographique sur papier salé représentant l'escalier du château de Blois) et pl. 41 (photolithographie Poitevin des Halles de Paris en construction).

6. César Daly, op. cit., vol. 45, 1888, col. 24 et suiv. et pl. 1. 
7. Épreuve acquise en décembre 2012 auprès de la librairie Plantureux et conservée au musée d'Orsay sous le numéro d'inventaire PHO 200623129.

8. D'abord un tampon AdB, puis un petit monogramme DB.

9. Peut-être est-ce ainsi à la suite de la demande de Daly relayée par Pascal que l'image fut réalisée.

10. La principale publication accordant une importance significative à De Bonis reprend cette idée dans son titre. Voir Bruce Lundberg, Maria Francesca Bonetti et al., Steps of the Beaten Path. Nineteenth-Century Photographs of Rome and its Environs, Milano, Edizioni Charta, 2007.

11. Ces photographies sont actuellement conservées au musée-château de Nemours et elles apparaissent, sans attribution, sur le portail Arago. Il s'agit notamment des épreuves portant les numéros d'inventaire 110,112, 113, 114, 116, 117, 119, 120, 121, 122, 123, 124, 125 et 126.

12. Francis Wey, Rome, description et souvenirs. Ouvrage contenant 346 gravures sur bois dessinées par nos plus célèbres artistes et un plan de Rome, Paris, Librairie Hachette, 1872. L'ouvrage, qui avait d'abord paru en feuilleton dans Le Tour du Monde entre 1868 et 1870, a ensuite connu plusieurs éditions et une traduction en langue anglaise.

13. Voir: Lucia Cavazzi, Anita Margiotta et Simonetta Tozzi (dir.), An Englishman in Rome. 1864-1877. The Parker Collection in The Municipal Photographic Archives, Roma, Regione Lazio, 1990 ; Peter J. Holliday, The Fascination with the Past. John Henry Parker's Phootgraphs of Rome, San Bernardino, California State University, 1991.

14. Étude Binoche et Giquello, vente aux enchères du 17 avril 2013, Hôtel Drouot, salle 4, lot 63 [consulté le 11 novembre 2013].

15. Voir à ce propos Piero Becchetti, La Fotografia a Roma dalle origini al 1915, Rome, Colombo, 1983, p. 290 et Bruce Lundberg, «A Note about the Photographic Techniques and Identification of the Photographs ", dans Lundberg Bonetti 2007, p. 200 et suiv.

16. Ces trois clichés portent respectivement les numéros 1212,1219 et 1201. S'il ne nous a pas été possible de dresser l'inventaire complet des photographies de la série concernant le palais de la Chancellerie, les épreuves repérées portent des numéros qui varient entre 1195 et 1219, soit 25 prises de vue différentes.

17. Aujourd'hui dans la collection Bruce et Delaney Lundberg que je remercie de me l'avoir signalé.

18. Piero Becchetti, La Fotografia a Roma dalle origini al 1915, Rome, Colombo, 1983, p. 347.

19. Adriano De Bonis, Regola dei cinque ordini di architettura di Giacomo Barozzi da Vignola, Florence, A spese degli editori, 1851 ; Adriano De Bonis, Trattato di prospettiva pratica per disegnare dal vero adattato al intelligenza di tutti, Florence, A spese degli editori. Thénot, lui-même élève de JeanThomas Thibault, auquel il avait succédé comme responsable du cours de perspective de l'École des beaux-arts de Paris s'inscrivait ainsi dans la filiation directe des architectes artistes qui gravitaient notamment dans l'entourage de Percier et Fontaine.

20. Voir à ce propos Jean-Philippe Garric, « De Percier et Fontaine à De Bonis : vues de la cour de l'ancien palais Mattei à Rome ", dans Mélanges offerts à Pierre Pinon, Paris, Picard, 2014. 
INDEX

Index géographique : Italie, Rome

Mots-clés : Académie de France à Rome, architecture, photographie, Villa Médicis

Index chronologique : XIXe siècle 


\title{
Le fonds photographique des architectes Ballu (INHA)
}

\author{
Hélène Bocard
}

1 Le fonds des architectes Théodore Ballu (1817-1885) et Albert Ballu (1849-1939), acquis en 2007 par l'INHA ${ }^{1}$, comprend des archives écrites (notes de voyages), des dessins, des plans, des cartes postales et 545 photographies datables entre 1869 et 1912. Pour une grande partie, ces photographies ont été prises ou collectées par les Ballu à l'occasion de voyages en France et dans différents pays d'Afrique et d'Europe; d'autres sont à mettre en rapport avec des chantiers de restauration d'Albert Ballu, notamment en Charente, où il exerça comme architecte en chef des Monuments historiques. Par commodité, on peut les classer en deux grandes catégories: les photographies «de voyage " et les photographies «de travail». Dans les deux cas, on trouve des photographies professionnelles émanant de maisons de renommée internationale (Alinari, Anderson, Brogi, Laurent, Sebah et Joaillier) ou de photographes moins connus établis en région (Graziani à Bastia, Léon Rosse à Saint-Malo ou Didier Fellot à Angoulême); des photographies réalisées par les Ballu eux-mêmes (plus probablement Albert) ou leur entourage; d'autres enfin sont anonymes, parmi lesquelles certaines ont pu être récemment attribuées.

2 Cet ensemble, constitué par les architectes eux-mêmes, illustre leur intérêt pour la photographie et l'usage qu'ils en faisaient aux côtés de pratiques plus traditionnelles comme le dessin, pour lequel Théodore Ballu avait une véritable passion: "Ses rares loisirs, Th. Ballu les occupait à des aquarelles et surtout à des dessins d'après nature qui sont des merveilles d'exactitude et de rendu. Les beautés du paysage le passionnaient en même temps que les monuments du passé l'attiraient. Aussi quelle moisson de croquis et de dessins au retour de ses voyages, de ses séjours au loin, de ses moindres excursions hors de Paris! Il laisse des cartons pleins de souvenirs qui évoquent pour les siens des moments heureux en même temps qu'ils sont des cuvres d'art qui auraient suffi à la réputation de tout autre artiste $e^{2}$. " Les nombreux dessins présents dans le fonds de l'INHA en témoignent ${ }^{3}$. 


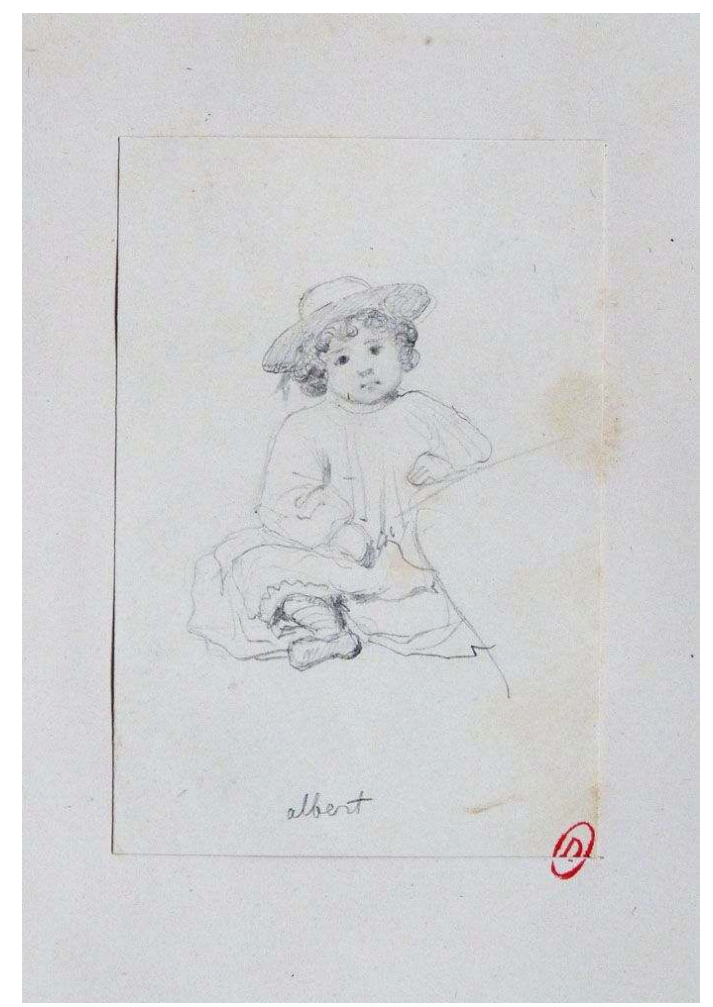

Fig. 1 : Théodore Ballu, Portrait d'Albert Ballu enfant, dessin au crayon.

3 Hormis la qualité intrinsèque de certaines épreuves, beaucoup de photographies permettent de documenter les vies des architectes, leurs parcours professionnels, leurs relations : photographies de la Villa Médicis où Théodore Ballu fut pensionnaire de 1841 à $1844^{4}$; photographies du domaine de Servanes à Mouriès où résidait l'architecte Henry Révoil (1822-1900) ${ }^{5}$; ou encore les photographies liées à des chantiers de restauration $^{6}$. La majeure partie d'entre elles a pour sujet des monuments (vues d'ensemble, détails, décors); mais on trouve aussi quelques portraits (portraits orientalisants de l'atelier Garrigues, à Tunis; portraits d'Arlésiennes), des scènes de genre (petits métiers ou scènes de rue au Caire) et des paysages. On ajoutera quelques unica comme des personnages au bord de la mer ou un boulevard parisien sous la neige.

L'intérêt de l'ensemble réside également dans son organisation, qui semble relever d'une véritable entreprise familiale. Si le classement géographique des photographies par département et par pays est vraisemblablement l'œuvre d'Albert, la présentation des photographies, collées sur des feuilles de papier fin (groupées par deux pour les petits formats), légendées au crayon, parfois datées et signées d'un monogramme ( $A B$ ou TB), fut pensée par les deux architectes. On trouve, dans les années 1870, des documents signés et datés de l'un ou de l'autre. La présence de leurs initiales semble indiquer qu'ils sont alors les auteurs de la photographie figurant sur la feuille. Les photographies professionnelles ont parfois subi le même traitement et dans certains cas la légende ou les éléments (cachet, numéro de série) qui auraient pu permettre leur identification ont disparu, la photographie ayant été découpée. Les photographies « de travail » sont parfois associées à des plans, à des dessins ou à des cartes postales collées sur une même feuille. 


\section{Photographies de voyages}

5 Une grande partie des photographies décrites ici a été prise ou collectée à l'occasion de voyages en France, en Afrique ou en Europe, sur lesquels les archives nous apportent quelques informations. Pour Albert Ballu, on recense des voyages en Italie (1874, 1878, 1890), aux Pays-Bas (1873), en Angleterre (1874), en Belgique (1876), en Espagne et au Maghreb (1879), en Égypte (1881-1882), à Malte (1882), en Grèce et en Turquie (1890), en Roumanie (1885), que l'on peut mettre en relation avec des archives écrites, des dessins, des photographies. La douzaine de photographies de l'abbaye de Westminster ${ }^{7}$ a sans doute été prise lors d'un voyage à Londres en juillet 1874, au cours duquel Albert Ballu visita de nombreux monuments et musées ${ }^{8}$. La même année, en septembre et octobre 1874, la famille Ballu partit en Italie pendant deux mois, peut-être pour fêter le mariage récent d'Albert avec Marguerite Houël. Le peintre Jean-Jules Antoine Lecomte du Noüy (1842-1923), ancien élève de Gérôme, les accompagnait ${ }^{9}$. Ils visitèrent notamment Turin, Milan, Pavie, Brescia, Vérone, Vicence, Venise, Padoue, Bologne, Ravenne, Florence, Naples, Pompéi, la Sicile. Le journal de voyage, tenu par Théodore Ballu (document de 13 pages), relate les détails de leur séjour et maintes anecdotes: visites de musées et de monuments (musée égyptien de Turin, mosaïques de Ravenne, fresques de Gozzoli), repas, trajets. Il mentionne parfois l'exécution de croquis, mais jamais de photographies. Pourtant, la forêt de pins de Ravenne, évoquée dans le journal, fait l'objet de plusieurs épreuves; certaines photographies furent sans doute achetées sur place comme la vue par Brogi de la cathédrale de Florence couverte d'échafaudages.

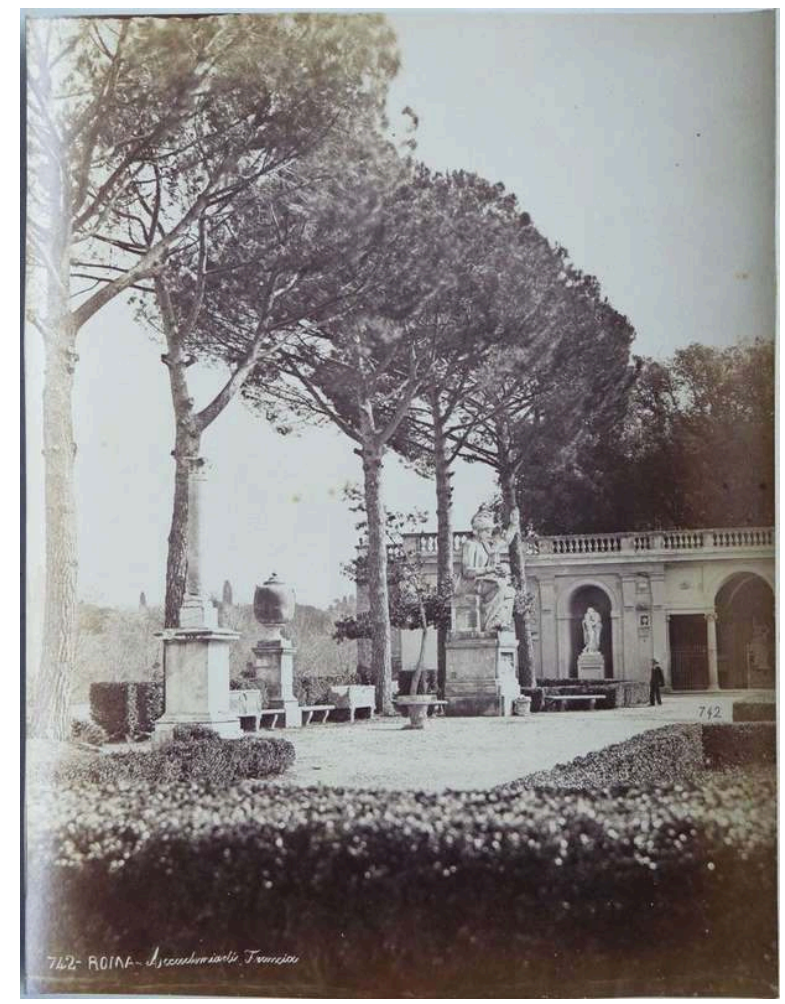

Fig. 2 : Adriano De Bonis (attribué), Vue de la Villa Médicis, tirage sur papier albuminé.

6 D'autres photographies d'Italie, rapportées d'un voyage en 1878, montrent la région de Gênes, le lac majeur et le lac de Côme (photographe Nessi). 
7 À l'automne 1879, Albert Ballu se rendit en Espagne puis au Maghreb. Sur la cinquantaine de photographies d'Espagne, on relève une belle série de douze épreuves de Casiano Alguacil (1832-1914), photographe de Tolède, montrant les rues étroites de la vieille ville, à la perspective accentuée, incluant souvent des personnages ou une charrette dans le champ de vision.

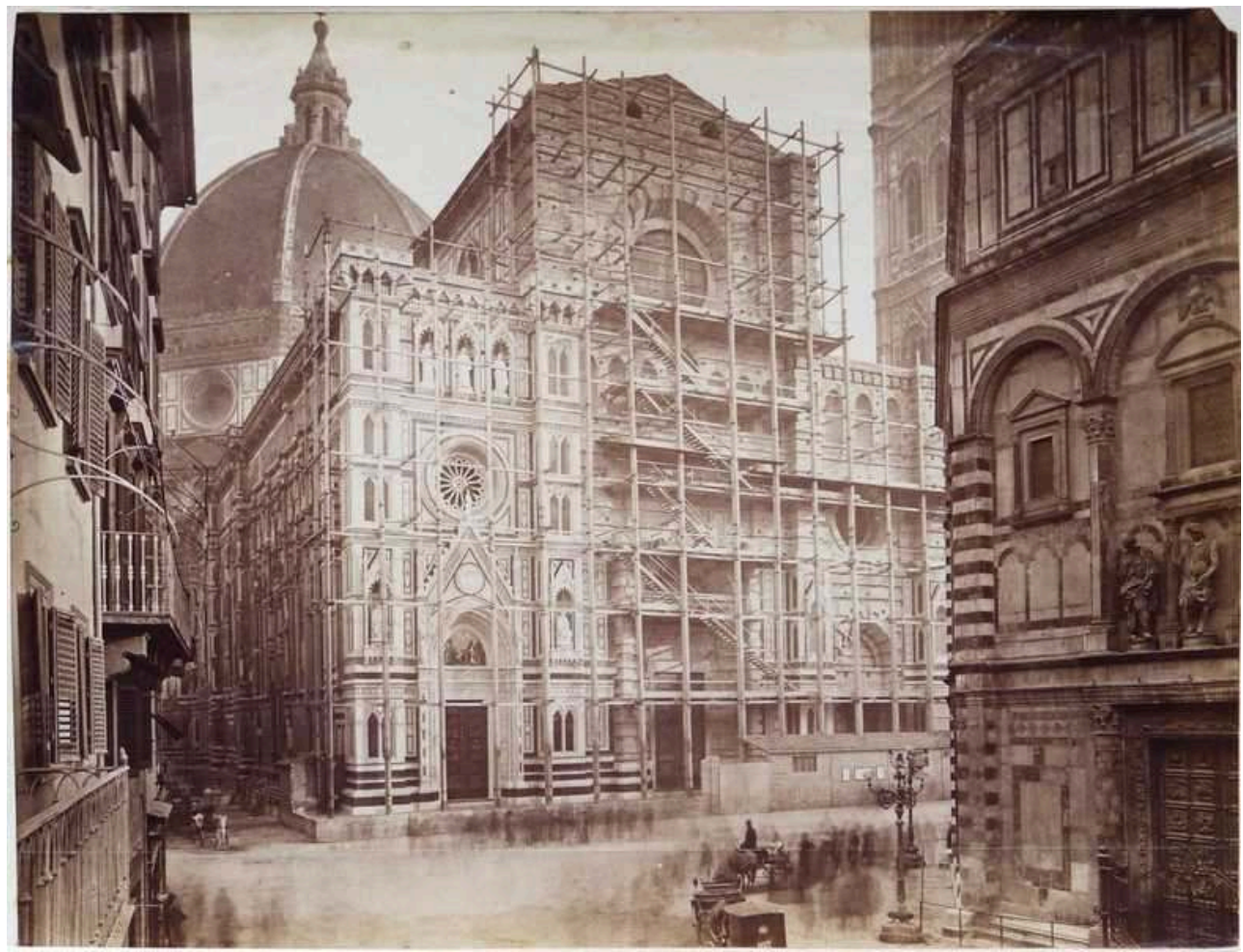

Fig. 3 : Carlo Brogi, La cathédrale de Florence échafaudée, tirage sur papier albuminé

8 Ces photographies ne sont pas sans rappeler celles d'Adolphe Terris dans le vieux Marseille. On en trouve également une dizaine d'autres prises par la maison Laurent (monuments, façades et intérieurs d'églises ou de palais de Madrid, Huelva, Jerez, Granada, Burgos...). Certaines d'entre elles, diffusées par des éditeurs français comme Giraudon, rue Bonaparte, ou J. Kuhn, rue de Rivoli, ont pu être achetées à Paris. On trouve aussi le cachet sec «A. C. Champagne, rue de Rivoli » sur des vues par Neurdein (ND) ou Léon et Lévy (LL) : intérieurs d'églises ou de couvents de Cordoue, Saragosse, Séville, Valence. Quelques vues de Gibraltar évoquent le passage entre l'Espagne et la côte africaine.

9 La Tunisie est représentée par 24 photographies : vues de Kairouan et de Tunis (rues, mosquées), portraits de l'atelier Garrigues, l'un des premiers installés à Tunis. Une vue de Béja, publiée en 1888 dans la revue le Tour du monde sous la forme d'un dessin, a pu être attribuée également à Garrigues. 


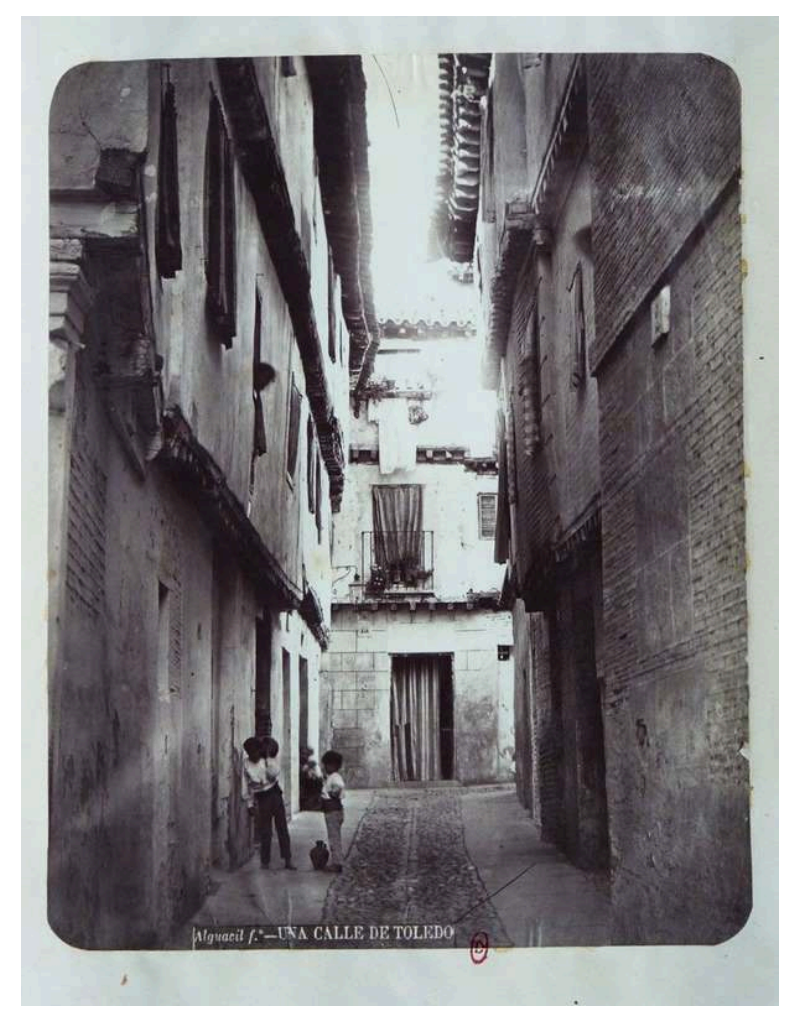

Fig. 4 : Casiano Alguacil, Une rue de Tolède, tirage sur papier albuminé.

10 Le Maroc est présent avec sept beaux tirages anonymes, peut-être d'Albert Ballu luimême (vues de Tanger). Si le fonds de l'INHA comprend des dessins d'Algérie ${ }^{10}$, on ne trouve aucune photographie de ce pays, où Albert Ballu exerça pourtant comme architecte diocésain puis comme architecte en chef des Monuments historiques à partir de 1889 avant de prendre, en 1903, la tête du nouveau service d'architecture chargé de l'étude et de la direction des travaux des édifices publics d'Algérie ${ }^{11}$.

11 L'ensemble concernant l'Égypte est l'un des plus importants, avec 88 épreuves: architecture d'époque pharaonique, architecture islamique, scènes de genre ou petits métiers. Beaucoup sont anonymes, d'autres sont signées ou attribuables à des photographes professionnels largement diffusés dans le bassin méditerranéen et audelà, comme Hippolyte Arnoux ou Sebah et Joaillier. Quelques-unes, plus rares, ont pu être attribuées à Benjamin Fachinelli (actif de 1877 à 1893), photographe italien qui s'installa au Caire au début des années 1880 ; il travailla notamment pour le comité de conservation des monuments de l'art arabe afin de fixer les rues, les édifices et monuments appelés à disparaître ${ }^{12}$. 


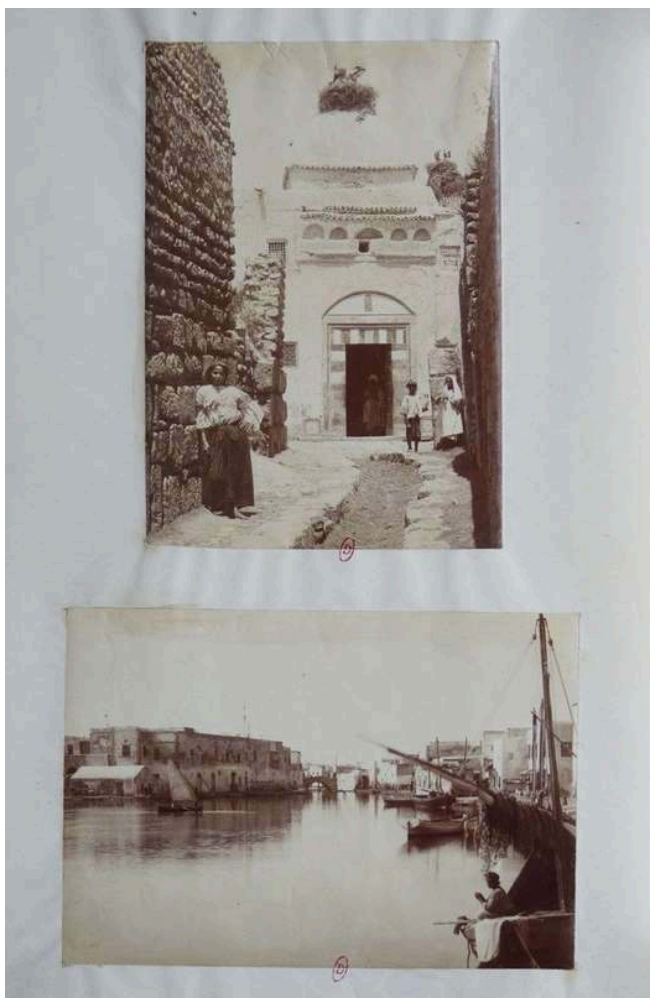

12 Fig. 5 : J. Garrigues, Deux vues de Béja en Tunisie, tirages sur papier albuminé.

On ne sait comment Albert Ballu, présent au Caire à la fin de l'année 1881, se les procura $^{13}$.

Un autre ensemble conséquent concerne la Turquie (une dizaine d'épreuves sur Brousse, une soixantaine sur Constantinople), où Albert Ballu séjourna en avril 1890. Pour l'essentiel, il s'agit de tirages de la maison Sebah et Joaillier, premier atelier de la capitale ottomane fondé par Pascal Sebah, auquel succéda son fils Jean, associé en 1888 à Polycarpe Joaillier. Elles montrent des monuments, des intérieurs de mosquées, des cimetières, des tombeaux, des décors en faïence et des vues de la ville.

Enfin, on signalera un ensemble significatif de dessins et de photographies concernant la Belgique, où Albert Ballu construisit le palais de justice de Charleroi (1875-1881). Ceux-ci proviennent de plusieurs voyages entre 1873 et 1878 : monuments et paysages d'Audenarde, Bruges, Charleroi (Boulevard central), Gand (maison des bateliers), Liège, Namur, Mons, Tournai.

Parmi les pays représentés en plus petit nombre, on notera quatre tirages sur le Portugal, signés de la maison Léon et Lévy (LL) (monuments de Braga, Lisbonne, Porto); quelques photographies d'églises et de monastères roumains sans doute prises par Albert Ballu lui-même ${ }^{14}$; une vue de Malte (1882) par le photographe Agius ou encore quatre vues de Dresde (Léon et Lévy et anonymes) ${ }^{15}$.

\section{Photographies de travail}

17 Les photographies que nous évoquerons maintenant couvrent une période plus large, de 1869 à 1909 environ (dates inscrites) et concernent des travaux menés par Albert Ballu sur des édifices français. Elles sont pour la plupart associées à d'autres documents 
(plans, calques, croquis, cartes postales) qui attestent à chaque fois d'un travail de l'architecte sur le monument.

Dès 1869 , on trouve plusieurs dessins et photographies d'un même édifice, qui figurent toutefois en tant que documents dissociés (l'église Saint-Leu de Taverny). En 1873, la basilique Notre-Dame d'Espérance à Mézières, dans les Ardennes ( $\mathrm{XVI}^{\mathrm{e}}$ et $\mathrm{XVII}^{\mathrm{e}}$ siècles), est représentée par une photographie qui la montre très endommagée (une grande partie des toitures fut détruite pendant le siège de 1870) et par un dessin signé « $A B$ ", daté d'août 1873, qui en propose une restitution. De la même année date une feuille composée par Albert Ballu sur Château-Thierry à partir de trois documents : un dessin de la tour Balhan, un dessin de l'intérieur d'une salle gothique dite " casemate » et une petite photographie prise d'un point élevé montrant une vue d'ensemble de la ville avec la tour Balhan. Ce type de composition qui associe divers documents sur un même support se retrouve en maints exemples dans le fonds. Parfois, les documents associés ne concernent pas tous le même édifice, comme pour Châlons-sur-Marne où sont associés deux photographies de l'intérieur de la cathédrale et un dessin d'un bâtiment au bord d'une rivière (1873). De la même époque, une feuille comprend au centre une photographie de la façade de la cathédrale Notre-Dame de Senlis, et, disposés autour, des dessins de détails d'architecture (ornement, culot, baie) relevés sur des monuments de Creil ou de Senlis ainsi qu'une partie en ruines du château voisin de Montépilloy. L'hôtel de ville de Dreux est représenté par une photographie (extérieur) et par des dessins de l'escalier; le mont Saint-Michel par une vue d'ensemble du site avec un personnage posant et un détail d'une baie gothique. À Taverny, trois photographies donnent à voir l'ensemble ainsi que des détails de l'église Saint-Leu.

Le plus souvent, la photographie montre l'ensemble de l'édifice, et le dessin, par nature plus analytique, reproduit des détails (église de Jouy-le-Moustier, 1873). À Dinan, des dessins reproduisent l'ensemble et des détails (appareils, culs de lampe) de la porte du Jersual. Une photographie signée Léon Rosse en montre également une vue d'ensemble. La ville de Dinan est représentée par plusieurs photographies de Rosse, dont un panorama et une vue des remparts ; deux petites photographies plus tardives, du début $\mathrm{du} \mathrm{Xx}^{\mathrm{e}}$ siècle, ainsi que des plans et des dessins témoignent du travail de Ballu comme architecte des Monuments historiques dans la ville bretonne.

Parmi les épreuves que l'on peut attribuer à Albert Ballu, certaines témoignent de réelles qualités esthétiques comme deux photographies prises à Cluny conservées avec des dessins datés de 1873. 


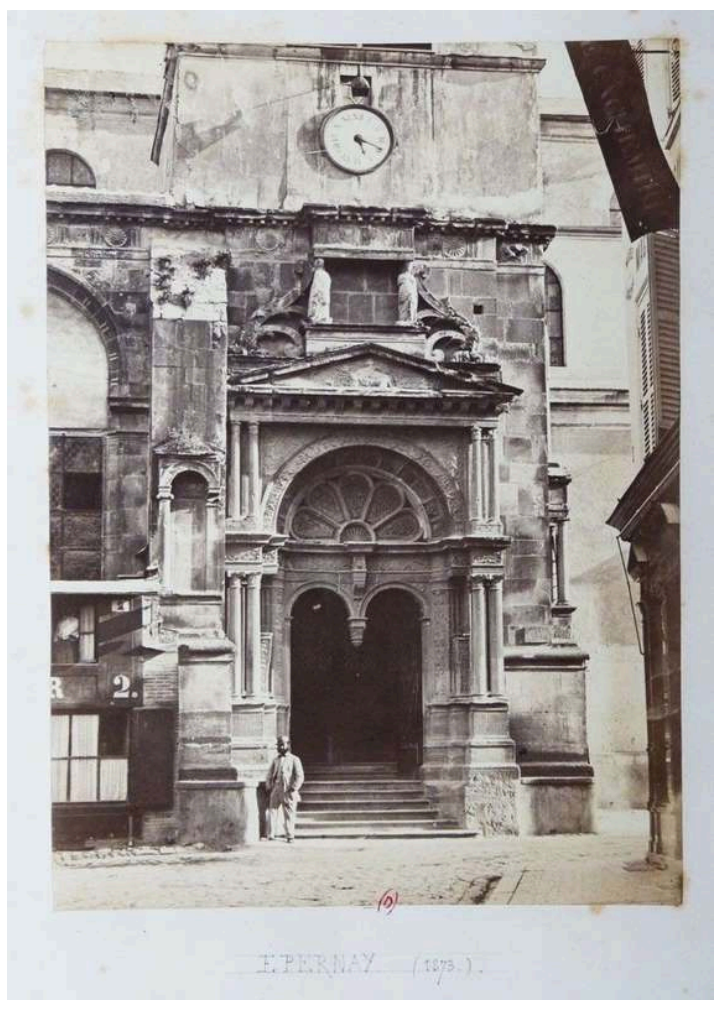

21 Fig. 6: Anonyme (Théodore Ballu ?), Portail de l'église Notre-Dame d'Épernay, tirage sur papier albuminé.

22 Souvent, des personnages posent devant le bâtiment : à l'hôtel Marisy à Troyes (1885) ou en 1873 à Épernay, devant le portail Renaissance de l'ancienne église abbatiale Notre-Dame, seul élément alors conservé de l'édifice, reconstruit en 1825 par Édouard Deperthes ${ }^{16}$.

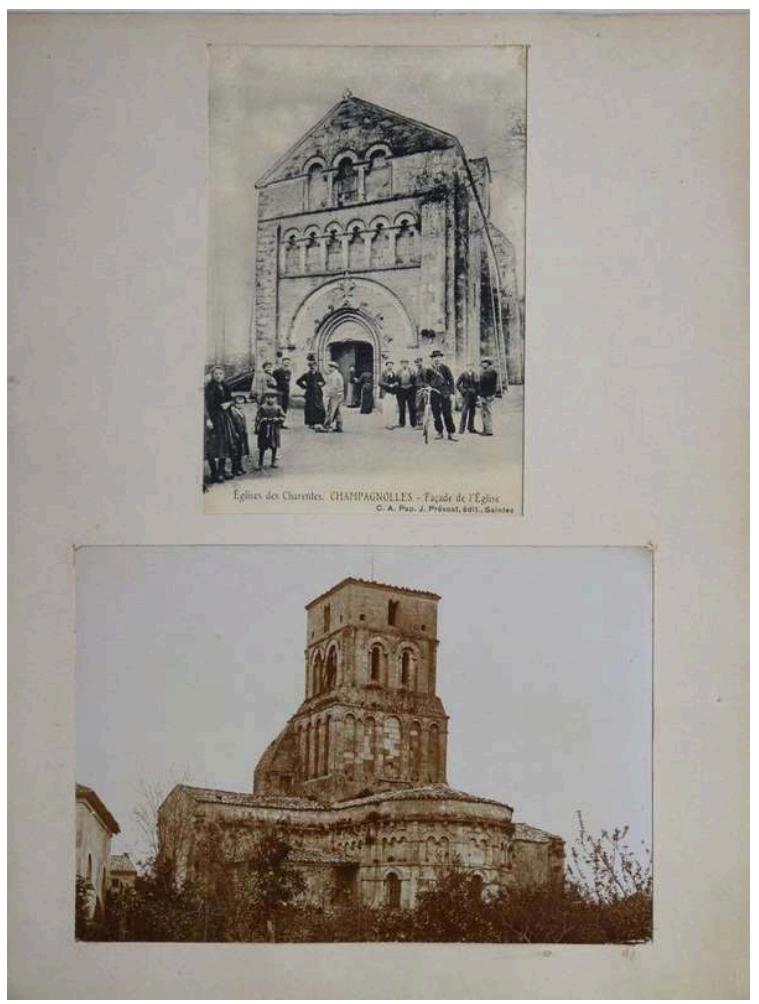


Fig. 7 : Anonyme (Albert Ballu ?), Église de Champagnolles (Charente), carte postale et photographie, tirage sur papier albuminé.

Un même dossier peut mêler photographies professionnelles et photographies d'amateurs. C'est le cas pour le château de Pierrefonds, qui contient des dessins réalisés par Albert Ballu datés de 1868, des photographies d'amateurs et deux autres de format ovale portant le cachet d'Albert Dupré, photographe à Compiègne. Des photographies de paysages enrichissent parfois les dossiers : vue de rivière à Dreux, personnages au bord de la mer (peut-être la famille de l'architecte) à Dinard. Cette pratique qui consiste à associer dessins et photographies existe aussi chez Théodore Ballu (on trouve, par exemple, ses initiales et la date de 1877 sur un ensemble de sept photographies des Baux de Provence).

Les plus tardives (1901-1909) concernent l'activité d'Albert Ballu comme architecte en chef des Monuments historiques dans le département de la Charente, où il fut chargé de dresser un inventaire des monuments à restaurer, de juger de la qualité des restaurations en cours et de l'état de leur avancement ${ }^{17}$. Si la plupart sont des églises, on trouve aussi les fortifications de Brouage ou le théâtre gallo-romain des Bouchauds (photographies datées de 1901 accompagnées d'éléments de correspondance portant sur la restauration du théâtre). On y découvre des photographies, des plans et des relevés d'édifices exécutés par Albert Ballu lui-même mais aussi par des architectes locaux en charge de ces restaurations. Si quelques églises du département étaient déjà protégées au titre des Monuments historiques, comme celle de Montmoreau, photographiée en 1851 par Gustave Le Gray pour la Mission héliographique, beaucoup ne le furent qu'au début du $\mathrm{xx}^{\mathrm{e}}$ siècle, à l'époque où Albert Ballu était en fonction, ou un peu après. Il restaura notamment les églises de Bourg-Charente (classée en 1913), Champagnolles (classée en 1910), Chadurie (inscrite en 1925), Geay (classée en 1907), Lichères (classée en 1903), Montmoreau (classée en 1846), Touvre, Trois-Palis (classée en 1886), Ventouse (inscrite en 1925).

Les notes conservées dans le fonds révèlent de nombreux déplacements à travers le département, en fonction des urgences. Albert Ballu a procédé à un classement de sa documentation par ordre alphabétique, sans doute plus pratique pour lui, auquel il pouvait ajouter des documents s'il retournait sur un lieu. Les photographies prises par l'architecte au cours de ces missions sont des petits tirages brillants de format carré, sans doute réalisées avec un appareil Kodak de petit format (premier modèle commercialisé en 1888 par George Eastman). D'autres furent prises par le photographe Didier Fellot, établi à Angoulême (églises de Chalais ou de Montmoreau).

On trouve souvent, dans cet ensemble, associés aux photographies, plusieurs documents sur un même support : cartes postales, plans, dessins. Un même édifice peut être illustré par plusieurs photographies, comme l'église de Lichères, représentée par une vue de l'intérieur, très détérioré, et trois vues de l'extérieur. Parfois, une même feuille comprend des vues de deux édifices différents : sont ainsi réunies les églises de Trois-Palis et de Ventouse; ou celles de Villognon (portail roman) et de Voulgezac (chevet).

Photographies et cartes postales sont associées dans certains dossiers comme celui de Brouage : une photographie petit format montre un détail des fortifications et une carte postale donne à voir les fortifications en ruines (avec fléchage à l'encre rouge pour indiquer le sujet de la photographie précédente); ou l'église de Champagnolles: 
une carte postale éditée à Saintes montre la façade de l'église avec des personnages posant et une photographie montre l'arrière de l'église, vue de trois quarts ${ }^{18}$.

D'autres fois, il s'agit d'un plan et d'une photographie associés sur un même support: l'église de Geay, illustrée par un plan et une photographie de la façade latérale; ou l'église de Chadurie, représentée par un plan et une photographie de la façade.

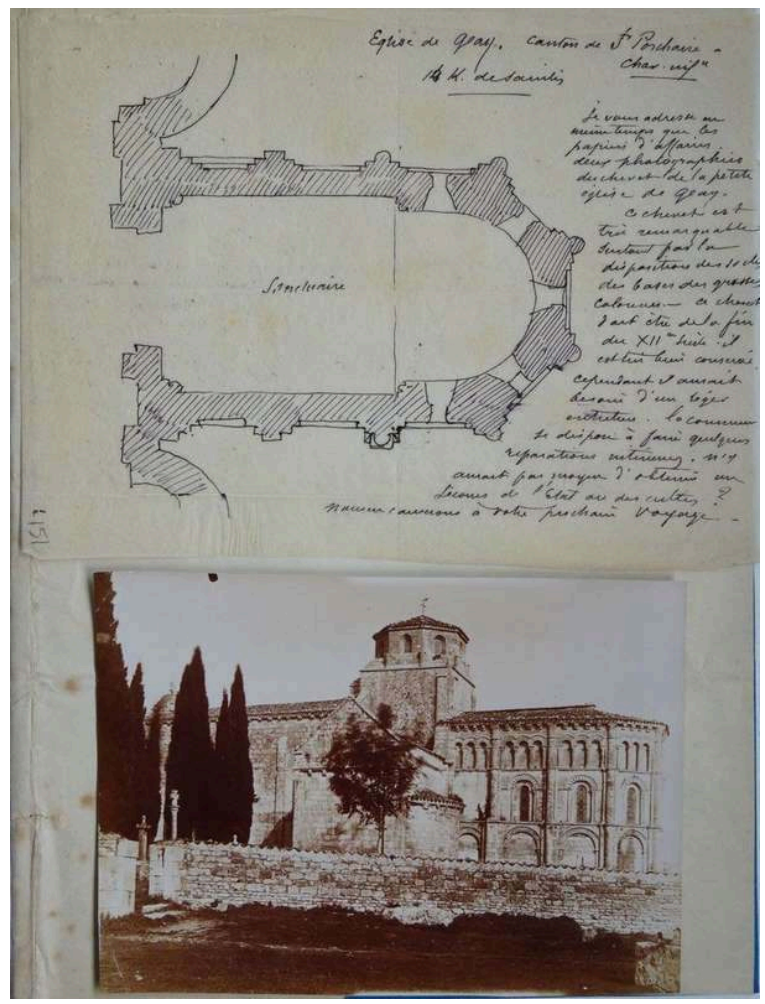

Fig. 8: Albert Ballu, Plan de l'église de Geay (Charente), dessin et anonyme (Albert Ballu ?), Église de Geay, tirage sur papier albuminé.

Les documents ainsi associés sont souvent complémentaires : la carte postale montre une vue d'ensemble ou une vue de l'extérieur et la photographie montre un détail ou une vue de l'intérieur. La couverture photographique des édifices se veut souvent systématique (extérieur, intérieur, ensemble, détails). Comme celle de Lichères, l'église de Chaix est représentée par trois photographies de l'extérieur et deux de l'intérieur ; ou l'église de Chermignac, illustrée par une carte postale et quatre photographies, le tout complété par une brochure, publiée en 1909.

Aux documents iconographiques s'ajoutent parfois des notes écrites comme pour l'église de Chadurie (notes rédigées en août 1902). Le plus souvent, les notes manuscrites, à l'encre, apposées sur les documents eux-mêmes, concernent des travaux en cours comme pour l'église d'Écoyeux où les photographies montrent les échafaudages (dossier contenant tous types de documents : bleu d'architecte, calques, photos, croquis, carte postale); ou pour l'église de Bourg-Charente (quatre photographies montrant le monument en ruines avec des notes sur les travaux en cours).

33 Les photographies sortent parfois du genre strict de la photographie d'architecture, incluant des personnages ou des festivités (l'église de Colombiers, photographiée un jour de fête, ou l'abbaye de la Couronne en ruines avec deux femmes posant). Des paysages apportent parfois une note bucolique, comme à Lichères, où l'on trouve, en 
plus de deux vues de l'église, deux paysages de rivière avec un homme dans une barque (septembre 1909).

En Charente, Albert Ballu pouvait satisfaire son goût pour l'art roman, qu'il partageait avec son père ${ }^{19}$. Dans la préface de l'ouvrage d'Emile Proust, Ce qu'il faut voir dans la Charente-Inférieure ${ }^{20}$, il notait : "Le public connait trop peu ce bel art saintongeais du $\mathrm{XII}^{\mathrm{e}}$ siècle ; le style roman, personne ne l'ignore, dérive du byzantin. » L'art byzantin, quant à lui, fait une large place à la mosaïque, ce dont témoigne là encore le fonds de photographies des Ballu, qui comprend des reproductions de mosaïques de Daphni ou de la basilique de Parenzo en Istrie, ainsi que de Ravenne, Rome, Venise, ou encore de Sicile ou de Turquie. Ce goût pour la mosaïque et pour le décor mural coloré (aquarelles reproduisant des décors de l'Alhambra) peut être mis en relation avec certaines réalisations d'Albert Ballu en Algérie, de style néo byzantin ou néo mauresque (cathédrale et gare d'Oran, casino de Biskra).

Le fonds Ballu conservé à l'INHA et les photographies qu'il contient reflètent l'activité mais aussi le goût et les sources d'inspiration des deux architectes pour leurs propres réalisations. En plus de l'intérêt iconographique, la présentation et le classement méthodique de l'ensemble attestent de la volonté des deux architectes d'organiser leur documentation, de conserver la trace de leurs voyages et de leurs travaux; il restitue aussi, en filigrane, l'esprit d'une famille.

\section{NOTES}

1. L'ensemble fut acquis en vente publique le 21 mai 2007 à Chinon. Il est conservé sous la cote Archives 112 (11 cartons). Un classement et un pré-inventaire ont été effectués en 2008par Flavie Seznec de Montgolfier, auteur d'un mémoire de maîtrise sur L'architecture des églises de la SainteTrinité et de Saint-Ambroise par Théodore Ballu (Paris IV, 2002, dir. Bruno Foucart et Simon Texier). En 2011, une expertise a été menée sur les photographies concernant l'Égypte par Ola Seif, responsable des collections photographiques de l'université américaine du Caire, en séjour à l'INHA (InVisu). Enfin, en 2012, Noémie Latte, étudiante à l'École du Louvre, a saisi les notices des photographies du carton $\mathrm{n}^{\circ} 11$, riche en photographies de divers pays. Les notices sont consultables sur la base Agorha de l'INHA. Le classement à l'intérieur des cartons est géographique, par département ou par pays.

2. Paul Sédille, "Théodore Ballu architecte », Bulletin mensuel de la Société centrale des architectes, 1886 (supplément), p. 394-407 (nécrologie). En 1846, de retour à Paris après avoir séjourné à la Villa Médicis (il reçut le prix de Rome en 1840) puis à l'École française d'Athènes, Théodore Ballu exposa une série d'aquarelles au Salon de peinture (vues de l'intérieur de Saint-Pierre de Rome et de monuments antiques d'Athènes).

3. Environ 370 dessins par Théodore, réalisés entre 1841 et 1877, et 240 par Albert, réalisés entre 1868 et 1890 (architecture, paysages, portraits).

4. Ces photographies, prises dans les années 1860 , se retrouvent dans la collection de Sir John Henry Parker conservée à l'INHA ; à noter cependant que les tirages du fonds Ballu, sur carton épais, sont postérieurs. 
5. Ces deux photographies d'objets antiques conservés dans le domaine de Servanes, collées sur une feuille signée «TB » datée de 1877, ainsi que deux dessins signés «TB sept. 1879 ", attestent de liens amicaux entre les deux familles. Plus tard, Albert Ballu réalisa le buste d'Henry Révoil pour le monument commémoratif qui fut érigé dans le jardin de la Fontaine de Nîmes, ville où Révoil vécut une partie de sa vie.

6. Pour Théodore Ballu, dont la carrière s'est déroulée presque entièrement à Paris (en 1860 il est nommé dans le nouveau service d'architecture de la Ville de Paris dirigé par Victor Baltard et en 1871 il est inspecteur des édifices de la Ville de Paris), seules les photographies de la Villa Médicis peuvent être mises en rapport avec son parcours professionnel. Les monuments qu'il a contribué à construire ou à restaurer (Sainte-Clotilde, Tour Saint-Jacques, la Trinité, Saint-Ambroise, l'Hôtel de Ville) ne sont pas représentés dans le fonds, à l'exception d'une vue de la Tour SaintJacques qui semble plutôt provenir d'archives d'Albert Ballu.

7. Sans cachet ni signature, avec une légende et un numéro inscrits au dos des feuilles sur lesquelles elles sont collées, elles présentent des défauts et maladresses et furent sans doute prises par Albert Ballu lui-même.

8. Le dossier contient des notes écrites au crayon avec le détail du séjour, du $1^{\mathrm{er}}$ au 8 juillet 1874 , et un dessin signé « $\mathrm{AB} 1874$ ».

9. On rappellera brièvement la composition de la famille Ballu. Théodore Ballu épousa en 1847 Claire Orsel (1824-1914) avec laquelle il eut trois enfants : Albert (1849-1939), marié en mai 1874 à Marguerite Houël (1849-1915); Roger (1852-1908), qui fit une carrière politique (en 1875, il intègrele cabinet du préfet de la Seine, puis devient, en 1883, inspecteur général des Beaux-arts ; il fut ensuite maire de Gournay-sur-Marne, conseiller général puis député de Seine-et-Oise de 1902 à 1906) et Cécile (1860-1922), mariée en 1884 au sculpteur Jean Idrac (1849-1884), décédé prématurément.

10. Dessins signés $A B$ et datés d'octobre et novembre 1879 montrant des paysages d'Alger, Constantine, Oran ainsi que des personnages (juif marocain).

11. Il conduisit les fouilles de Timgad, Djemila, Théveste au cours desquelles il prit de nombreuses photographies dans les années 1890; voir l'ensemble important conservé à la médiathèque de l'Architecture et du Patrimoine (base Mémoire du ministère de la Culture). En tant qu'architecte, il acheva la cathédrale d'Alger, construisit le casino de Biskra, la gare d'Oran, la cathédrale de Constantine. Il construisit aussi les pavillons de l'Algérie à l'Exposition universelle de 1889 et à l'Exposition coloniale de Marseille en 1906. Le projet de la cathédrale d'Oran (1908-1912) témoigne d'une utilisation précoce du béton avec le concours des frères Perret. Sur la carrière d'AlbertBallu, voir les notices dansFrançois Pouillon, Dictionnaire des orientalistes de langue française, Paris, Karthala éditions, 2008, p. 43-44; et Nabila Oulebsir, Les usages du patrimoine. Monuments, musées et politique coloniale en Algérie (1830-1930), Paris, Maison des Sciences de l'homme, 2004, p. 322.

12. Des photographies de Facchinelli sont également conservées dans la photothèque Jacques Doucet, à la bibliothèque de l'INHA ; un travail est en cours sur Facchinelli par Maryse Bideault (CNRS, laboratoire InVisu) et Ola Seif, du Caire.

13. Dans une lettre datée du 7 octobre 1881, adressée par Christophe-Edouard Mauss, architecte du ministère des Affaires étrangères, au consulat général de France en Égypte au sujet de la venue d'Arthur Ali Rhoné, attaché à la mission scientifique du Caire dirigée par Gaston Maspero, il est question d'Albert Ballu, qui doit se rendre en Égypte avec sa femme, pour raison de santé ; l'auteur de la lettre ajoute que «M. Ballu est allié de M. Rhoné. » (Archives du ministère des Affaires étrangères, carton 491: Le Caire, particuliers et autorités diverses) ; document communiqué par Maryse Bideault, que nous remercions.

14. Albert Ballu est à l'origine du palais de justice de Bucarest (1886-1895), achevé par l'architecte roumain Ion Mincu, pour lequel il obtint le prix Duc en 1888. 
15. L'une porte le cachet humide de «Albert Hautecoeur (maison Martinet), 12 bd des Capucines » et deux autres le cachet humide « Kunsthandlung S. Jacops 16 passage KÖLN ».

16. Le portail, seule partie conservée de l'ancienne église démolie en 1909, a été remonté dans un square à l'emplacement de l'ancienne abbaye dont il constitue aujourd'hui le seul vestige connu sous le nom de "portail Saint-Martin» (la Charité de saint Martin figurait dans la niche rectangulaire du sommet). Il constitue sans doute le monument le plus célèbre de la ville, plusieurs fois gravé et photographié au XIX ${ }^{\mathrm{e}}$ siècle. La photographie du fonds Ballu montre les cariatides de la niche du haut encore très lisibles, alors que la pierre a complètement fondu de nos jours. Édouard Deperthes (dont Théodore Ballu fut le collaborateur sur les chantiers de l'église de la Trinité à Paris puis de l'hôtel de ville) s'est inspiré de ce portail pour l'entrée principale de la basilique de Sainte-Anne d'Auray. Nous remercions vivement Bernard Ducouret, conservateur du patrimoine au service régional de l'Inventaire en Champagne-Ardenne, pour ces précisions.

17. On ne connaît pas la date de son début d'activité en Charente, mais en 1898 il travaille déjà sur l'église de Montbron (informations communiquées par Jean-Charles Forgeret, de la médiathèque de l'Architecture et du patrimoine, que nous remercions).

18. Sur l'utilisation des cartes postales par les architectes, voir l'ouvrage récent de Luis Burriel Bielza, Le Corbusier : la passion des cartes, Bruxelles, Mardaga, 2013.

19. En 1897, Albert Ballu publia un ouvrage sur Le monastère byzantin de Tébessa (éditions Ernest Leroux). On rappellera, parmi les réalisations de Théodore Ballu, l'église Saint-Denis d'Argenteuil ou Saint-Ambroise à Paris, qui s'inspirent du style roman.

20. Ouvrage publié à La Rochelle (impr. N. Texier) en 1905, écrit à partir des notes de Charles Dangibeaud (1851-1935), conservateur des musées de Saintes.

\section{AUTEUR}

\section{HÉLÈNE BOCARD}

Institut national d'histoire de l'art 


\title{
Louis Émile Durandelle (1839-1917) : un photographe au service des architectes
}

\author{
Charlotte Leblanc
}

«Photographe du nouvel opéra et des élèves de l'École des Beaux-arts », comme il se désignait lui-même dans les annonces publicitaires, Louis Émile Durandelle collabora entre 1860 et 1890 avec de nombreux architectes comme Charles Garnier, Édouard Corroyer, Ambroise Baudry, Paul Abadie, Edmond Guillaume, Juste Lisch ou Jean Louis Pascal. Il produit ainsi une documentation photographique des chantiers parisiens de construction et de restauration. On lui doit entre autres un important corpus photographique fournissant de précieuses informations au sujet des travaux de l'opéra Garnier, du Sacré-Cœur de Montmartre, du Mont-Saint-Michel, du Comptoir national d'escompte de la ville de Paris, de la gare Saint-Lazare, de l'Hôtel-Dieu, de la tour Eiffel, du palais du Louvre ou encore du musée des beaux-arts d'Amiens. Les collections françaises sont particulièrement riches en tirages sur papier albuminé produits par la Maison Durandelle (Bibliothèque nationale de France, bibliothèque des Arts décoratifs, Médiathèque de l'architecture et du patrimoine, bibliothèque historique de la ville de Paris, École nationale supérieure des beaux-arts, etc.). Pourtant, Louis Émile Durandelle reste mal connu. Ainsi, ses photographies illustrant les étapes de la construction de l'opéra Garnier ou de la tour Eiffel sont très présentes dans l'imaginaire collectif de l'histoire urbaine de Paris, mais rares sont ceux qui en connaissent l'auteur. 


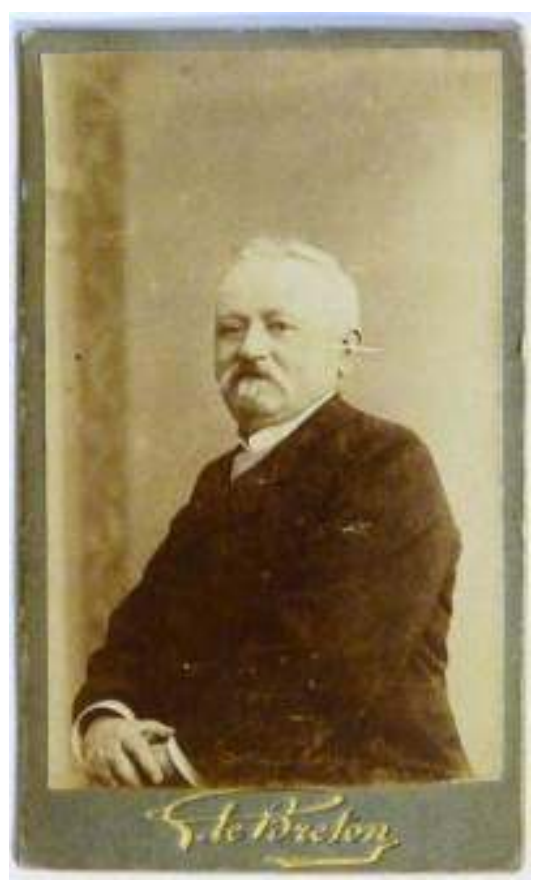

Le Breton, Portrait photographique de Louis Émile Durandelle, s. d., collection particulière.

2 L'intérêt suscité pour le travail de Louis Émile Durandelle est assez récent. C'est en juillet 1984 que parut le premier article important sur ce photographe sous la plume d'Elvire Perego: «Delmaet et Durandelle, ou la rectitude des lignes. Un atelier du $\mathrm{XIX}^{\mathrm{e}}$ siècle $^{1} »$. Suivirent l'article d'Ulrich Keller ${ }^{2}$ sur les photographies de l'opéra de Paris en 1988 et la thèse de Carlo Benini ${ }^{3}$ abordant également l'œuvre de Durandelle pour Charles Garnier. Enfin, un important travail de recherche fut effectué par l'historien et photographe Claude Baillargeon dans une thèse soutenue en 2002 : «Religious Fervor and Photographic Propaganda: Durandelle's Anatomical Studies of the Sacré-Cœur de Montmartre $^{4} »$. Elle permit de mieux connaître le parcours du photographe et d'éclairer plus particulièrement son rôle dans le financement de l'édification du Sacré-Cœur de Montmartre $^{5}$. Nos présentes recherches s'inscrivent dans cet approfondissement progressif de la connaissance sur le photographe, sous la forme d'une thèse commencée en 2011 à l'École pratique des hautes études sous la direction de Jean-Michel Leniaud, portant plus particulièrement sur les rapports entretenus par Louis Émile Durandelle avec l'architecte Édouard Corroyer ${ }^{6}$.

3 Afin de replacer Louis Émile Durandelle dans le contexte de la photographie d'architecture au milieu du XIX $x^{e}$ siècle, nous souhaitons nous appuyer sur un important article de Barry Bergdoll, écrit en 1994 à l'occasion de l'exposition Édouard Baldus?7. Il permet de comprendre combien la commande des photographies de chantier par les architectes et l'administration était devenue une pratique courante à cette période. Louis Émile Durandelle n'était donc pas novateur en ce sens. Cependant, il semble avoir reçu les commandes photographiques de nombreux chantiers majeurs de cette période. En effet, certains architectes ont fait appel à lui à plusieurs reprises dans leur carrière. Nous nous interrogerons ici sur les raisons de ce succès auprès des architectes, notamment des architectes parisiens. Était-il réputé pour sa technique photographique? Quel usage les architectes faisaient-ils de ses photographies?

4 À ce stade de la recherche, il est seulement possible d'émettre des hypothèses sur le statut et le rôle de Louis Émile Durandelle auprès des architectes. Ces suggestions 
s'appuieront plus particulièrement sur le rapport qu'il a pu entretenir avec Édouard Corroyer (1835-1904) $)^{8}$, architecte qui faisait un usage relativement varié de la photographie et qui, à ce titre, rend particulièrement intéressante la confrontation entre ces deux corps de métier: elle lui permit aussi bien de mémoriser un état historique d'un édifice que d'enregistrer les étapes de construction de ses bâtiments ou de promouvoir sa carrière et son œuvre.

\section{Fixer, mémoriser la ruine}

5 Louis Émile Durandelle est né à Verdun le 14 février 1839, de parents fabricants de cordes. On ne sait rien de sa jeunesse, ni de son éducation ou de son apprentissage de la photographie. A-t-il eu des maîtres? A-t-il commencé par peindre comme certains photographes de cette époque? A-t-il vu les photographies d'Édouard Baldus? On repère seulement la trace de Louis Émile Durandelle comme peintre en décoration à Paris en $1859^{\circ}$. Puis il apparaît comme photographe en 1860, à l'occasion de la destruction du château de Bercy (voir plus loin). Il a alors 21 ans. L'année suivante, il s'associe avec Hyacinthe César Delmaet pour fonder un atelier de photographie ${ }^{10}$. Il poursuit son activité de photographe industriel et commercial jusqu'en 1890. Puis, à 51 ans, il décide de se consacrer à des activités politiques et sociales. D'abord conseiller municipal des villes de Colombes puis de Bois-Colombes de 1892 à $1900^{11}$, il s'engage ensuite dans le développement de la Société philotechnique de Bois-Colombes, une association philanthrope destinée à former bénévolement des adultes à de multiples cours élémentaires. Membre du conseil d'administration dès 1891, il en devient le président en 1903 puis président honoraire en 1909 et obtient à ce titre les palmes académiques ${ }^{12}$.

6 À ses débuts, la Maison Delmaet et Durandelle ne semble pas s'être spécialisée dans un domaine particulier; elle propose dans ses premiers prospectus publicitaires un large choix de photographies, incluant des genres aussi divers que le portrait, la reproduction artistique ou la photographie juridique :

«Photographes du Nouvel opéra, des élèves de l'École des beaux-arts, comme par le passé, nous continuerons à nous occuper de la spécialité qui a contribué à nous faire connaître, c'est-à-dire la reproduction artistique, industrielle et commerciale tels que Bronzes, Tableaux, Dessins, Cristaux, etc. [...] reproduire soit un mur mitoyen en mauvais état, qui étant une cause de procès entre propriétaires, ralentit ou suspend les travaux en cours d'exécution; ces photographies sont pour la partie intéressée un relevé de l'état des travaux qui lui permet de faire valoir ses droits sans être obligé de faire faire une expertise [...] Nous nous transportons également en province pour reproduire les maisons de campagne, les usines, les châteaux dont on veut faciliter la vente par la publicité ou en faire des albums pour orner la table d'un salon. Portraits / Nous nous chargeons aussi d'exécuter les groupes soit à domicile, soit sur notre terrasse, dont la bonne disposition et l'étendue nous permettent d'opérer facilement et rapidement par tous les temps. Spécialité de portraits dits photo-émail, en carte de visite ordinaire depuis $10 \mathrm{fr}$. la douzaine, portraits de toute grandeur, portrait après décès ${ }^{13}$. »

7 Une spécialisation dans la photographie d'architecture semble surtout s'affirmer au fil des annonces publicitaires et des nouvelles commandes. Après le succès que lui vaut la campagne photographique du Nouvel Opéra, les commandes pour de nouveaux chantiers affluent et nous pouvons alors penser que Durandelle s'est progressivement spécialisé dans la photographie d'architecture par opportunisme professionnel. 


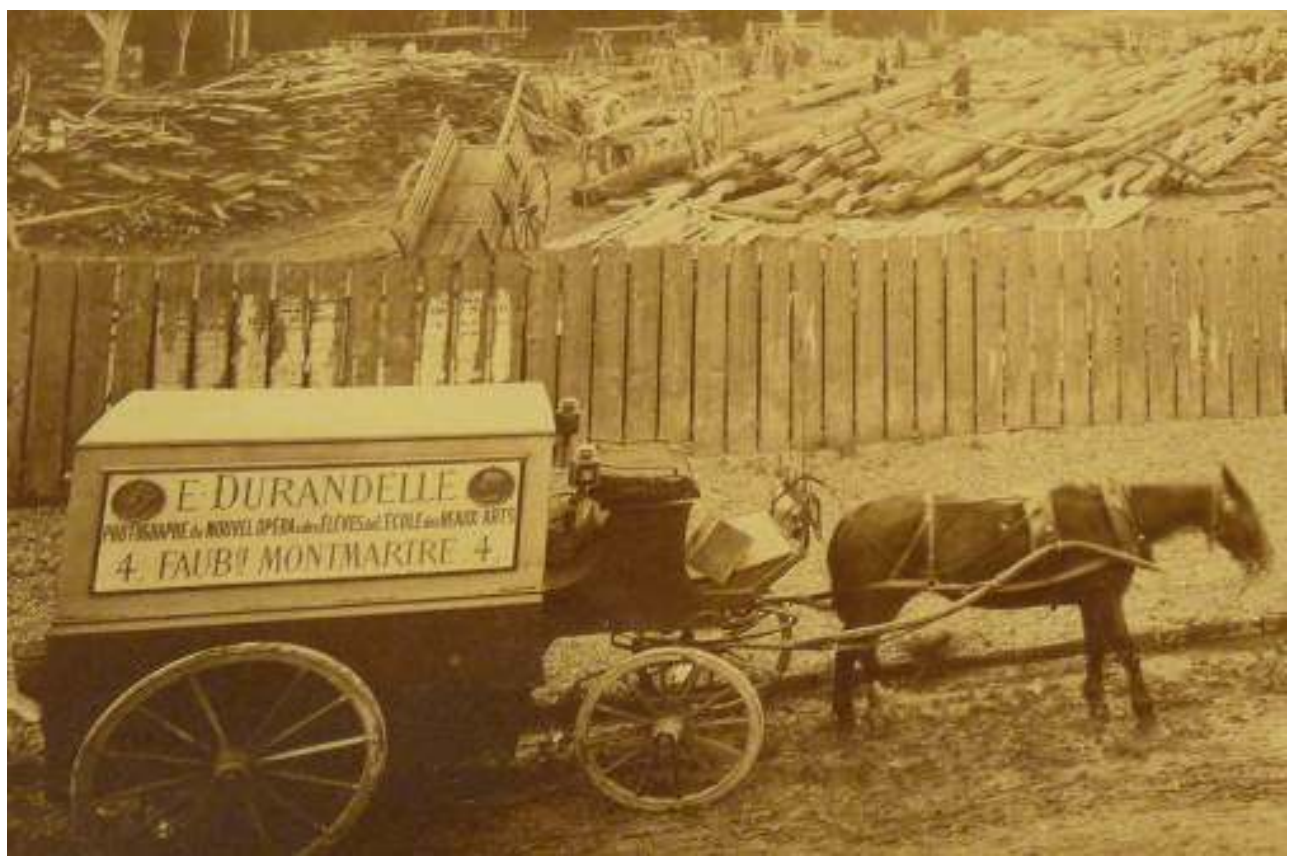

Louis Émile Durandelle, Attelage avec publicité de la Maison Durandelle (détail), s. d., tirage sur papier albuminé, bibliothèque des Arts décoratifs, $\mathrm{PHO} 4.1$

8 Le premier travail connu de Durandelle date donc de 1860. En raison de la construction du chemin de fer entre Paris et Lyon et de l'agrandissement des entrepôts de vins favorisé par l'annexion de la commune de Bercy, on décida de détruire le château de Bercy. Les intérieurs furent démantelés et vendus et on fit appel à des mouleurs, des peintres et des photographes pour conserver le souvenir du château. C'est peut-être le propriétaire du château de Bercy lui-même, le marquis de Nicolaï, qui commanda à Durandelle des photographies des boiseries provenant du grand salon et des plâtres de détails ornementaux. On ne sait quel usage exact fut fait de ces photographies destinées à enregistrer un élément du patrimoine sur le point de disparaître ${ }^{14}$.

9 Louis Émile Durandelle fut de nouveau sollicité pour documenter un édifice historique à l'occasion des restaurations du Mont-Saint-Michel, menées de 1872 à 1888 par Édouard Corroyer. Dès 1872, Viollet-le-Duc recommanda Corroyer sur ce chantier afin qu'il étudiât et signalât les éléments les plus intéressants de l'édifice dans le but d'obtenir une aide financière de la Commission des Monuments historiques pour la restauration de la Merveille. Édouard Corroyer exécuta ainsi une importante étude : travaux archéologiques, relevés et photographies ${ }^{15}$. La plupart des photographies de Durandelle présentent des vues générales du Mont-Saint-Michel ainsi que des détails architecturaux tels que la porte du roi, le cloître et les remparts. Certaines photographies montrent également l'installation des échafaudages et les débuts de la restauration entreprise dès le mois d'août 1875. Les photographies de Durandelle furent un outil de travail pour Corroyer comme en témoignent les annotations portées directement sur les tirages conservés aux archives départementales de la Manche ${ }^{16}$. Certaines d'entre elles furent retenues pour illustrer les ouvrages de Corroyer sur le Mont-Saint-Michel et sur l'histoire de l'architecture romane et gothique ${ }^{17}$. Il en réutilisa systématiquement les cadrages et les lignes de construction pour produire des gravures ensuite éditées dans ses ouvrages ${ }^{18}$. 


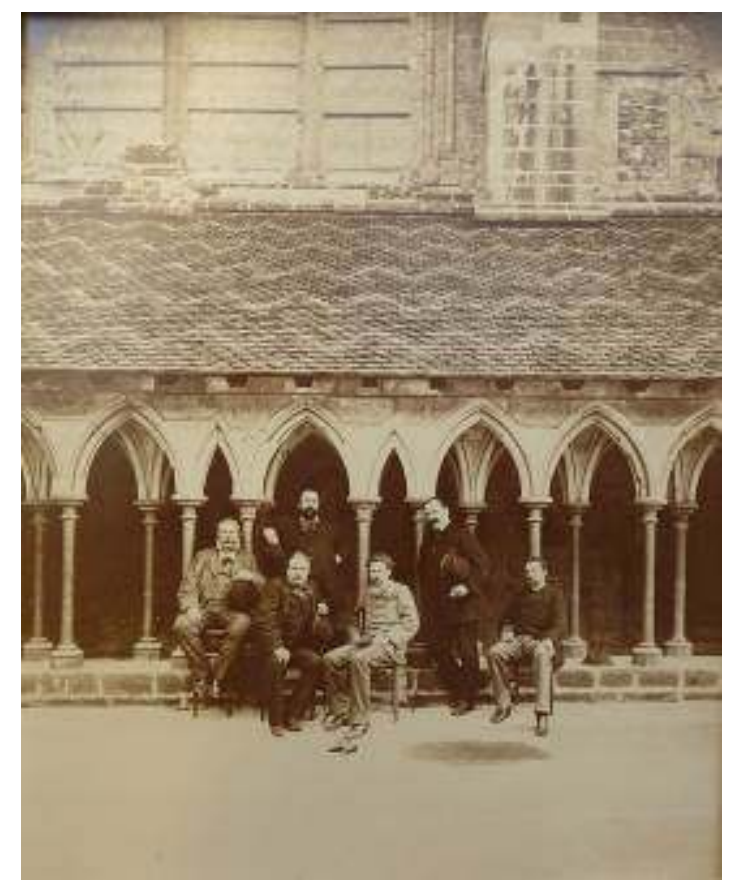

Louis Émile Durandelle, Équipe de restauration du Mont-Saint-Michel dans le cloitre (avec Édouard Corroyer), s. d., tirage sur papier albuminé, Bibliothèque nationale de France, Eo98 fol (1 ${ }^{\mathrm{e}}$ partie).

\section{Enregistrer les étapes de la construction}

10 À l'instar d'Édouard Baldus qui photographie à partir de 1855 la destruction puis la reconstruction de la grande galerie du Louvre de Lefuel, Louis Émile Durandelle a enregistré les étapes de construction de nombreux édifices, depuis leurs fondations jusqu'à l'achèvement complet du bâtiment. Il semble même que cette activité représente la partie la plus importante de sa carrière. Nous pouvons ainsi citer les photographies des chantiers de l'opéra de Charles Garnier, de l'Hôtel-Dieu, du théâtre de Vaudeville, de la Bibliothèque Nationale, de l'Exposition universelle de 1878, du théâtre de Monte-Carlo, du Comptoir national de l'escompte, de la gare Saint-Lazare, de la tour Eiffel, du palais des Machines, de la basilique du Sacré-Cœur et d'autres encore. 


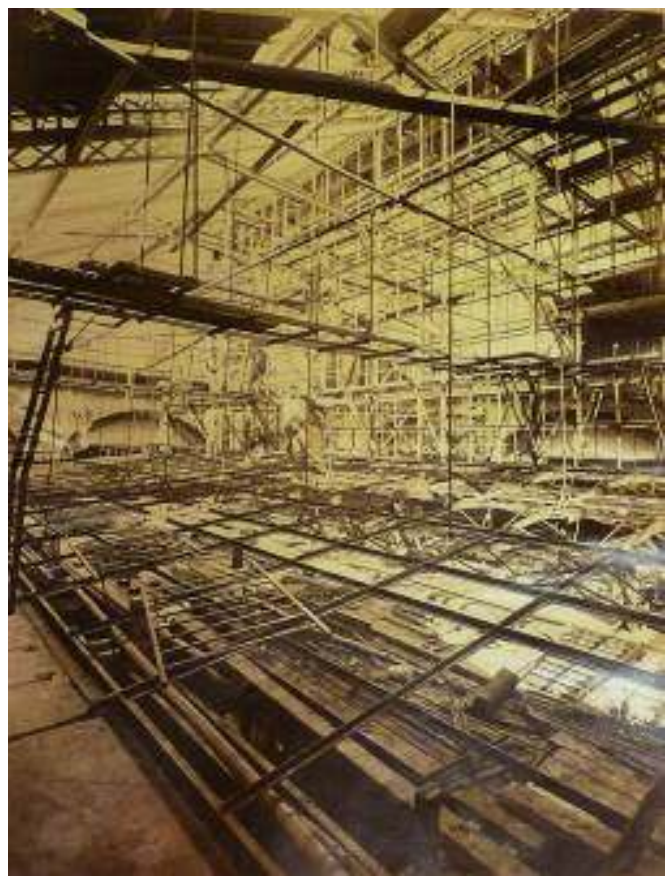

Louis Émile Durandelle, Vue des travaux de la verrière du Comptoir d'escompte de la ville de Paris, s. d., tirage sur papier albuminé, bibliothèque des Arts décoratifs, PHO 30.24.

11 Pour quelles raisons les architectes souhaitaient-ils enregistrer les étapes de la construction de leur édifice? Quel usage ont-ils fait de ces photographies? Cela varie sans doute en fonction du contexte de la commande. D'une part, ces photographies permettaient un suivi plus rigoureux du chantier pour les administrations qui finançaient les travaux. Elles ont aussi pu servir aux architectes eux-mêmes pour maitriser des chantiers longs et volumineux depuis leur bureau. Parfois, elles ont eu des usages plus spécifiques, comme celles du Sacré-Cœur de Montmartre, qui ont servi à des appels de dons afin de financer la construction de la basilique tout au long du chantier ${ }^{19}$.

12 Le succès de Durandelle auprès des architectes, pour enregistrer les étapes de construction de leurs édifices, s'explique en partie par sa grande maîtrise du rendu de la géométrie architecturale et sa capacité à limiter le problème des distorsions de perspective. Dans le rapport du jury de l'Exposition universelle de 1878 à Paris, le viceprésident de la Société française de photographie, Alphonse Davanne, insistait sur la difficulté majeure de la photographie d'architecture : «Pour obtenir une reproduction non déformée, il est certaines règles absolues auxquelles il faut se conformer ». Il recommandait l'usage de lentilles « capables d'embrasser un angle suffisant pour qu'on puisse les excentrer de manière à obtenir les parties hautes du monument sans inclinaison de la chambre noire » et félicitait ainsi Durandelle pour son travail : « Parmi les épreuves les mieux réussies en ce genre, nous citerons les reproductions de l'HôtelDieu de Paris et celui [sic] du mont Saint-Michel par M. Durandelle ${ }^{20}$.»

13 Corroyer semble également fidèle à Durandelle dans ses diverses commandes photographiques d'édifices tout au long de sa carrière pour ces mêmes raisons techniques: «M. Durandelle est déjà généralement connu des architectes pour la beauté des épreuves qui sortent de ses ateliers, aussi le recommandons-nous d'une manière toute spéciale aux lecteurs du Panthéon de l'Industrie ${ }^{21} »$. 


\section{Promouvoir une œuvre ou une carrière}

14 Durandelle semble n'avoir jamais publié lui-même ses photographies. En revanche, celles-ci étaient mises au service des architectes afin de faire connaître leur travail. Ses photographies d'architecture eurent donc parfois une postérité au-delà d'un simple usage à caractère administratif et constructif.

15 Ainsi furent-elles souvent utilisées par les architectes afin de présenter leurs derniers travaux à des confrères ou à des personnalités importantes. Il était en effet d'usage d'envoyer une photographie de son projet ou de son édifice construit accompagné d'une dédicace. Il s'agit parfois également d'envois de travaux des élèves de l'École des beaux-arts où Durandelle semble s'être fait une réputation. On trouve ainsi régulièrement dans des collections privées d'architectes et de sculpteurs des photographies de projets et travaux réalisés portant le timbre à sec de la Maison Durandelle. Il arrive également que ces tirages soient aujourd'hui conservés dans les collections nationales.

16 Les architectes pouvaient également promouvoir leur travail et leur carrière lors des différents salons organisés à Paris ou lors des expositions internationales et universelles. Traditionnellement, les architectes y présentaient des dessins et relevés de leurs édifices dans la lignée de l'enseignement de l'École des beaux-arts ou de l'Académie d'architecture. Ils pouvaient quelques fois ajouter des maquettes à leur présentation. Peu à peu, la photographie va également devenir un médium de promotion des architectes lors de ces salons. Édouard Corroyer notamment est précurseur dans cette pratique qu'il défendit âprement jusqu'à ce qu'elle soit admise au salon :

« Nous persistons à croire que cette manière [la photographie] de présenter les travaux d'architecture est la seule bonne et pratique, parce que le cliché et l'épreuve photographique deviennent la sanction de l'œuvre et permettent de la juger sous toutes ses formes, sans qu'elle soit traduite par des dessins ou des lavis trop complaisants. Nous espérons que la photographie sera admise désormais aux expositions annuelles ou triennales non plus seulement à titre de renseignement complémentaire, mais qu'elle aura conquis le droit de cité que lui ont valu les services qu'elle a rendus et qu'elle est appelée à rendre ${ }^{22}$. »

17 Les architectes ont également pu souhaiter diffuser leurs œuvres à un plus large public. Les photographies ont alors été utilisées dans le cadre de publications. Celles du chantier de l'Opéra de Paris par Durandelle ont ainsi été l'outil d'une véritable politique d'autopromotion de la part de l'architecte Charles Garnier s'attachant à défendre son opéra dans une monographie faite de textes, de gravures et de photographies (115 épreuves au total réparties dans quatre albums) ${ }^{23}$. Outre les publications d'Édouard Corroyer utilisant les photographies du Mont-Saint-Michel déjà citées, les photographies de Louis Émile Durandelle ont également été publiées sous forme de gravures par Hubert Rohault de Fleury (1828-1910), secrétaire général du " comité de l'œuvre du Vœu national du Sacré-Cœur de Jésus », alors chargé de la promotion de l'édification du Sacré-Cœur de Montmartre afin de récolter des fonds. Comme Claude Baillargeon l'a montré dans sa thèse, les photographies de Durandelle étaient reprises pour la publication du Bulletin de l'œuvre du Vœu national au Sacré-Cœur de Jésus destiné à une large diffusion. Il était alors davantage question de la promotion de l'édifice et du programme politique et religieux que de la carrière même de l'architecte Paul Abadie. 
Présent sur la plupart des grands chantiers parisiens du dernier tiers du xix siècle, Louis Émile Durandelle a donc côtoyé un nombre important d'architectes sans que son nom ne soit pour autant cité par ces derniers dans leurs publications ou qu'il figure dans leurs archives. L'enregistrement des étapes du chantier par la photographie étant perçu comme un processus mécanique, le statut de Louis Émile Durandelle restait ambivalent. Si la qualité de ses photographies était reconnue par tous, son identité et son rôle n'étaient pas mis en avant. Ce statut pose un problème pour la recherche car, ainsi, les archives d'architectes ne délivrent pas d'informations permettant d'identifier les relations entretenues avec le photographe. Par ailleurs, les archives de la Maison Durandelle n'ayant pas été retrouvées, il faut se tourner du côté des archives publiques pour tenter de comprendre son rôle sur les chantiers de construction. Or, il semble que son rapport au monde de l'architecture soit passé plus directement par la commande privée que par celle des institutions publiques de construction et de restauration.

L'approfondissement des recherches sur sa carrière dans le cadre de notre thèse de doctorat permettra, nous l'espérons, de palier en partie ce manque de sources et d'éclaircir davantage la nature de ses rapports avec les architectes contemporains.

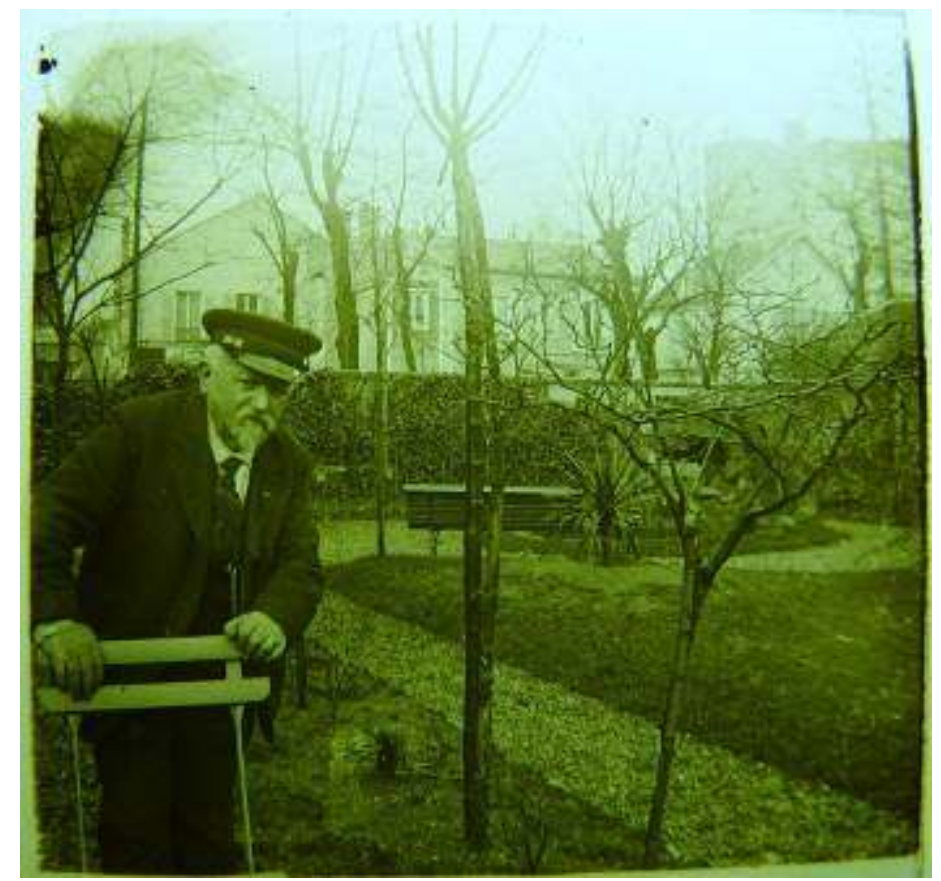

Anonyme, Portrait photographique de Louis Émile Durandelle, s. d., plaque de verre stéréoscopique, collection particulière.

\section{NOTES}

1. Elvire Perego, «Delmaet et Durandelle, ou la rectitude des lignes. Un atelier du XIX siècle », Photographies, $\mathrm{n}^{\circ}$ 5, juillet 1984, p. 54-83. 
2. Ulrich Keller, "Durandelle, the Paris Opera, and the aesthetic of creativity ", Gazette des Beauxarts, Janvier-février 1988, p. 109-118.

3. Carlo Benini, Anatomia di un edificio Louis Emile Durandelle fotografo e la costruzione dell'opera di Charles Garnier, 1864-1872, Bologne, Universita degli studi di bologna, facolta di lettere e filosofia, sous la direction de Mario Lupano, 2001.

4. Claude Baillargeon, Religious fervor and photographic propaganda: Durandelle's anatomical studies of the Sacré-Cour de Montmartre, thèse de doctorat de philosophie et d'histoire de l'art, Santa Barbara, University of California, sous la direction de Ulrich Keller, 2002.

5. On pourra également lire: Claude Baillargeon, "L'apport de la photographie à la construction", dans Jacques Benoist, Le Sacré-Cœur de Montmartre, un vœu national, Paris, Délégation à l'Action artistique de la Ville de Paris, 1995, p. 146-155 ; Claude Baillargeon, « Au service de la propagande du Sacré-Cœur: l'album de travail de Rohault de Fleury ", L'album photographique: histoire et conservation d'un objet, actes de colloque, Section française de l'Institut international de la conservation, Paris, 1998, p. 77-94; Claude Baillargeon, "Construction photography and the rhetoric of fundraising: the Maison Durandelle Sacré-Cœur Commission ", Visual Ressources, vol. 27, $\mathrm{n}^{\circ}$ 2, juin 2011, p. 113-128.

6. Charlotte Leblanc, La photographie au service de l'architecture : Louis-Émile Durandelle (1839-1917) et Édouard Corroyer (1835-1904), thèse de doctorat, Paris, École pratique des hautes études, sous la direction de Jean-Michel Leniaud. Ce travail a bénéficié d'une allocation de formation et de recherche de trois mois de la Direction générale des Patrimoines, ministère de la Culture et de la Communication.

7. Barry Bergdoll, «Une question de temps: architectes et photographes pendant le Second Empire ", dans Édouard Baldus, Photographe, cat. expo., New York, The Metropolitan Museum of Art, 1994 / Montréal, Centre Canadien d'architecture, 1995 / Paris, musée des Monuments français, 1995, Paris, RMN, 1994, p. 99-119.

8. Sur l'architecte Édouard Corroyer, voir Marie Gloc, Construire, restaurer, écrire. E. J. Corroyer (1835-1904), l'architecture dans tous ses états, thèse, Paris, École pratique des hautes études, 2003.

9. Conseil de révision: procès-verbal de délibérations, 1859, archives départementales de la Meuse, 1R 144. Nous remercions Monique Hussenot de nous avoir fourni ces précieuses indications.

10. Baillargeon 2002.

11. Délibérations du conseil municipal de Bois-Colombes, de 1892 à 1896, archives municipales de Colombes, 1D1/12 à 16; Politique communale de Bois-Colombes, archives départementales des Hauts-de-Seine, DO 9/57.

12. Centenaire de l'association philotechnique, archives privées, Société philotechnique de BoisColombes.

13. Prospectus publicitaire de la maison Delmaet Durandelle: «Changement de domicile. Agrandissement des Ateliers de photographie de la maison Delmaet Durandelle », vers 1872, archives Chevojon.

14. Certaines photographies de Louis Émile Durandelle pour le château de Bercy sont aujourd'hui conservées à la Bibliothèque nationale de France (série topographique) sous la cote VA-94-Folio $t$. 5. Deux albums de grande qualité sont conservés à la bibliothèque des Arts décoratifs (N $116 ; \mathrm{XC}$ 33). Une série est également conservée à la bibliothèque historique de la ville de Paris. Une photographie isolée se trouve, quant à elle, au musée d'Orsay sous la cote PHO 1983-35. Nous remercions Sylvie Aubenas de nous avoir fourni ces précieuses indications.

15. Marie Gloc, op. cit.

16. Archives départementales de la Manche, 28 Fi 1-240, Collection Édouard Corroyer. 1863-1888.

17. Édouard Corroyer, Description de l'Abbaye du Mont-Saint-Michel et de ses abords, Paris, Dumoulin, 1877 ; Édouard Corroyer, Guide descriptif du Mont-Saint-Michel, Paris, Ducher, 1883 ; Édouard Corroyer, L'architecture romane, Paris, Maison Quantin, compagnie générale d'impression et 
d'édition, 1888 ; Édouard Corroyer, L'architecture gothique, Paris, Librairies-imprimeries réunies, 1891.

18. Béatrice Krikorian et Charlotte Leblanc, Louis-Émile Durandelle : la mémoire du Mont Saint-Michel, cat. expo., Paris, bibliothèque des Arts décoratifs, 26 février-30 avril 2014, version numérique disponible sur le site internet des Arts décoratifs.

19. Baillargeon 2002.

20. Alphonse Davanne, Exposition universelle internationale de 1878 à Paris. Groupe II, Classe 12, Rapport sur les épreuves et les appareils de photographie, Paris, Imprimerie Nationale, 1880, p. 45.

21. Édouard Corroyer, « Photographie artistique et industrielle », Le Panthéon de l'Industrie, 16 mai 1880, no 164, p. 6.

22. Édouard Corroyer, "L'architecture à l'exposition nationale des Beaux-arts de 1883 ", Revue générale de l'architecture et des travaux publics, 1883, col. 266.

23. Charles Garnier, Le Nouvel Opéra de Paris, Paris, Ducher et Cie, 1878. Voir à ce sujet: Martin Bressani et Peter Sealy, "The Opera disseminated: Charles Garnier's Le Nouvel Opera de Paris (1875-1881) ", dans Stephen Bann (dir.), Art and the early photographic album, Washington, National Gallery of Art, New Heaven/London, Yale University Press, 2011, p. 197-219.

\section{AUTEUR}

\section{CHARLOTTE LEBLANC}

Archives nationales de France 


\title{
Architecture et photographie d'architecture au XIX ${ }^{\mathrm{e}}$ siècle au Portugal
}

\author{
Alexandra Trevisan et Maria Helena Maia
}

1 Au Portugal, l'histoire de la photographie est une discipline relativement récente. Aussi ses relations avec l'histoire de l'architecture doivent-elles être exposées avec prudence, les études sur le sujet étant encore peu nombreuses.

2 À l'exception d'Augusto da Silva Carvalho, qui a publié, en 1940, la première étude sur la photographie portugaise ${ }^{1}$, restée d'ailleurs la seule pendant quatre décennies, jusqu'en 1980, la recherche dans ce domaine se résume à quelques éléments ${ }^{2}$. Dans les années 1980 commence à se manifester un intérêt croissant pour le sujet, comme en témoignent quelques articles et études monographiques consacrés à des photographes, comme Frederick Flower, Carlos Relvas ou Joshua Benoliel ${ }^{3}$, notamment à travers l'activité de l'association ETHER, dont António Sena fut le principal promoteur ${ }^{4}$. Celle-ci organisa la première exposition rétrospective sur la photographie au Portugal de 1839 à 1992, dans la galerie ETHER, en 1992.

3 En 1991, António Sena publia la première histoire de la photographie au Portugal ${ }^{5}$, suivie, en 1998, d'une Histoire de l'image photographique au Portugal', qui comprend la publication simultanée d'un grand nombre d'images reproduites à partir d'originaux, permettant une vision d'ensemble jusqu'alors inédite. Récemment, Maria do Carmo Serén a réalisé une nouvelle synthèse ${ }^{7}$. Au-delà de ces travaux de référence, l'histoire de la photographie intéresse un nombre croissant de chercheurs, comme cela se manifeste aussi bien à travers des travaux universitaires que sur Internet ${ }^{8}$.

4 Malgré l'existence des archives photographiques nationales, institution à l'origine de l'actuel centre portugais de la photographie, la valorisation tardive de la photographie dans les milieux académiques et la reconnaissance tout aussi tardive de sa valeur patrimoniale contribuent à expliquer ce relatif silence historiographique autour de la photographie. À cela, il faut ajouter l'état de conservation souvent mauvais des photographies, faute de traitement convenable et de moyens adaptés. 
Quand la photographie est arrivée au Portugal, le pays sortait d'une période de guerre civile. Après la fin de la guerre civile avec la victoire libérale de 1834 sur la faction absolutiste, elle entra dans une période de relative stabilité.

L'invention de la photographie suscita au Portugal un enthousiasme presque immédiat, comme en témoignent plusieurs articles. Sa diffusion fut rapide et, dès 1840 , les premiers daguerréotypes furent réalisés. On connait une gravure du palais d'Ajuda, réalisée en 1840 à partir d'un daguerréotype.

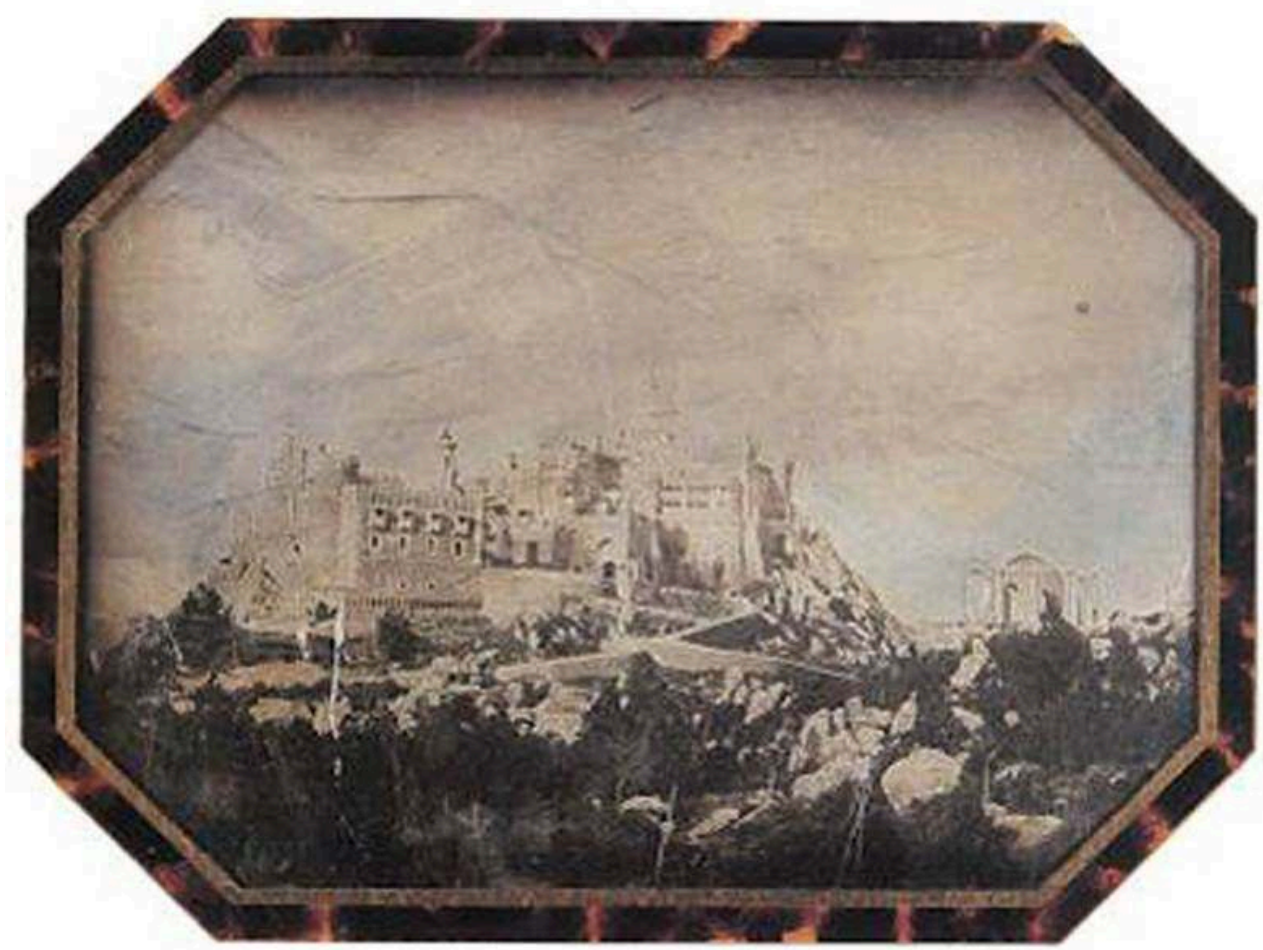

Fig. 1 : Vensceslaw Cifka, Palais de la Pena, Sintra, Portugal, 1846, daguerréotype.

7 Dès les origines, la photographie au Portugal est aussi bien le fait de photographes portugais que d'étrangers en voyage, qui séjournaient pour de longues périodes ou même s'installaient dans le pays. Nous distinguerons, parmi beaucoup d'autres noms, ceux de Wenceslau Cifka, Frederik Flower et le baron de Forrester, qui ont photographié l'architecture tout au long $\mathrm{du} \mathrm{XIX}^{\mathrm{e}}$ siècle. Ces photographes, majoritairement étrangers, ont parcouru le pays et se sont établis surtout dans les grandes villes, comme Porto, Lisbonne et Coimbra. Les premiers témoignages sont dus, pour l'essentiel, aux photographes ambulants et aux amateurs'.

8 L'évolution des procédés photographiques a entraîné une diversification des thèmes. Pendant les années 1860 , le daguerréotype restait le procédé préféré des photographes de studio, qui se consacraient essentiellement au portrait et travaillaient pour des clients qui cherchaient la précision et un raffinement de détails. D'un autre côté, le négatif sur papier devint dès 1850 le procédé préféré des photographes qui travaillaient en extérieur. Moins précis que le daguerréotype, mais plus léger et plus pratique à manipuler, il fut surtout utilisé par les photographes amateurs, qui avaient à cœur d'affirmer la valeur artistique du medium ${ }^{10}$.

Le collotype, utilisé par exemple par Frederick Flower, et le collodion humide, adopté par Forrester, ont amené ces photographes à pratiquer en extérieur. Les paysages, le 
patrimoine naturel et architectural, l'enregistrement des mœurs et coutumes ont pris de plus en plus de place dans leurs travaux. Si on ne connaît pas d'architecte photographe avant Possidónio da Silva, dont nous reparlerons plus tard, l'architecture a toujours eu sa place dans la photographie portugaise, qu'il s'agisse de nouveaux bâtiments, ou, plus fréquemment, du désir de documenter un patrimoine que l'on commence à mettre en valeur et à diffuser, notamment à partir de 1860, à travers des éditions facilitées par l'évolution des techniques d'impression et de reproduction (photogravure et zincographie).

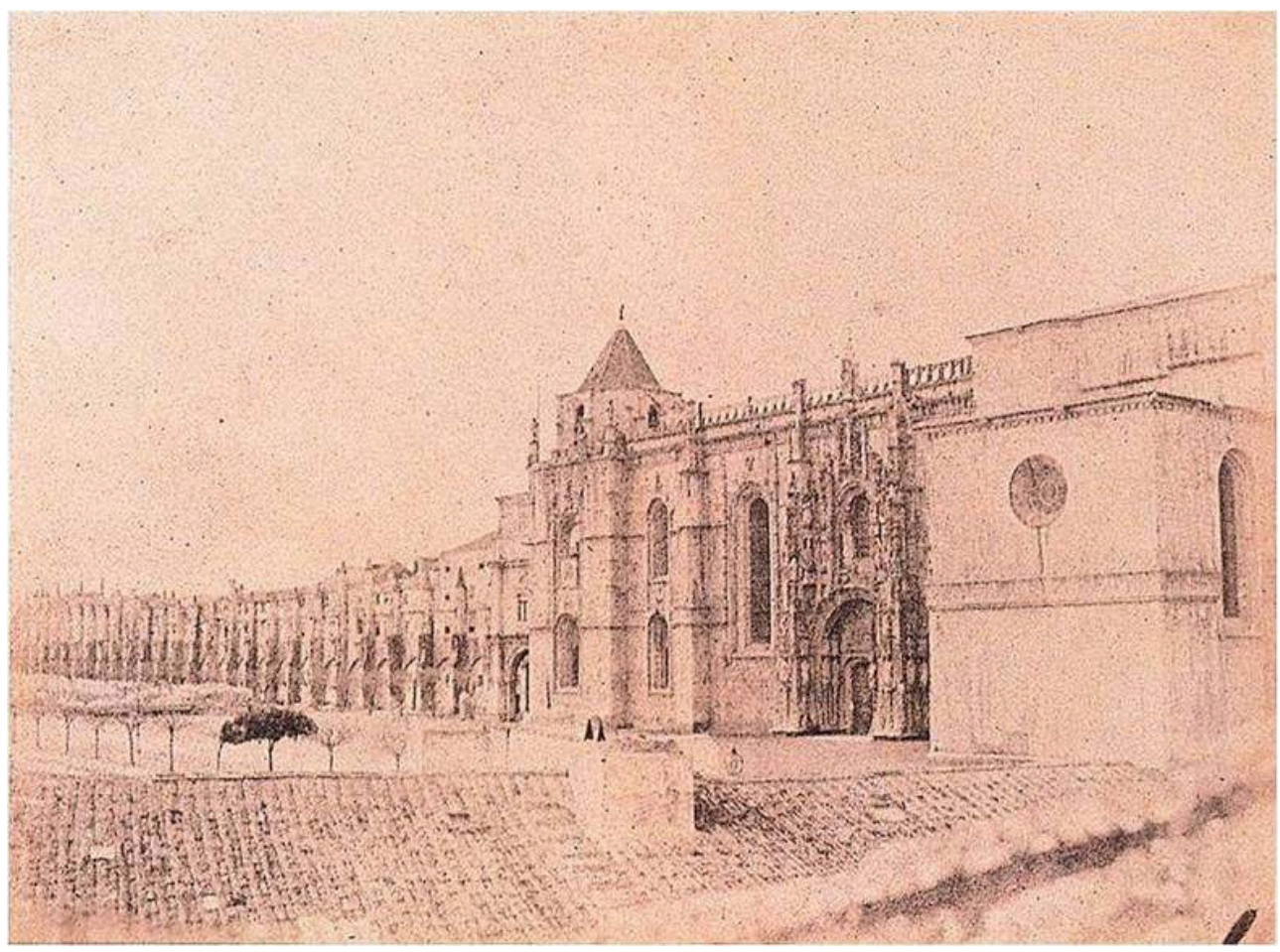

Fig. 2 : Possidónio da Silva, Monastère des hiéronymites à Belém, Lisbonne, vers 1862, papier salé, 19,7 x $26,8 \mathrm{~cm}$. Publié dans Revista Pittoresca e Descriptiva de Portugal com vistas photográphicas.

À remarquer que cet intérêt est directement lié à l'émergence de la notion de patrimoine dans les années 1840 et, parallèlement, à l'essor d'une presse périodique dont le lectorat ne cesse de s'accroître.

La première publication connue avec des vues panoramiques et des vues de bâtiments est l'album Le Portugal pittoresque et architectural dessiné d'après nature, édité par William Barclay vers 1841. Il ne parait pas avoir connu une large diffusion. En revanche, la Revista pittoresca e descriptiva de Portugal com Vistas Fotográficas [Revue pittoresque et descriptive du Portugal avec des vues photographiques], publiée entre 1861 et 1863 par Joaquim Possidónio da Silva a connu un grand succès dans les milieux culturels et artistiques portugais ${ }^{11}$.

12 S'agissant des architectes photographes, le premier fut Joaquim Possidónio da Silva (1806-1896) ; élève à Paris dans l'atelier de Percier et Fontaine, il fut le fondateur de l'Association des Architectes civils portugais (1863), puis de la Société royale des Architectes civils et des Archéologues portugais. Il a voué une grande partie de sa vie à l'archéologie et à la protection du patrimoine national, utilisant la photographie comme témoignage et comme moyen de diffusion dans les publications auxquelles il a contribué. 

qui fut exposée à Porto ${ }^{12}$. En 1848, quand il était encore un jeune architecte de la Casa Real, Possidónio da Silva commença à s'intéresser au patrimoine. Il pensa alors publier un album de dessins du Monastère des hiéronymites, "pour rendre le monument universellement connu », mais il rencontra des difficultés et abandonna le projet ${ }^{13}$. C'est surtout dans les années 1850 , après une incursion dans l'enseignement ${ }^{14}$ et sans doute insatisfait de sa carrière d'architecte, qu'il se consacra plus fréquemment au patrimoine et à l'archéologie ${ }^{15}$. Et à partir de 1858, il choisit la photographie pour s'aider dans son inventaire monumental. Bénéficiant d'une autorisation accordée par le roi Pierre V, qui soutenait son idée de mesurer et dessiner les monuments anciens les plus importants du Portugal, " en les classant suivant leur genre et en les comparant avec d'autres analogues ${ }^{16}$ ", il effectua ce qui paraît constituer le premier inventaire, sinon de tout le patrimoine architectural national, du moins d'une série d'édifices considérés comme significatifs à cette époque.

Bien que n'ayant pas connaissance du matériel graphique lié à ce travail, nous savons qu'au cours de l'année 1859 il a visité le couvent de Jésus à Setubal et qu'il dessina le plan de la cathédrale de Braga $^{17}$. Sont également documentées certaines visites effectuées les années suivantes, notamment en 1861 à Coimbra, où il prit « une vue photographique» du portail de la Sé Velha [ancienne cathédrale]et du monastère d'Alcobaça, dont il releva les plans, ainsi que celle qui l'amena à Porto en 1862, où il dessine le plan de l'église de Cedofeita ${ }^{18}$. Cela montre qu'il commençait à utiliser la photographie comme une alternative au dessin pour la représentation de l'architecture.

En effet, Possidónio da Silva publia à cette époque, avec l'appui de la Casa Real, dans le périodique Revista Pitoresca e Descriptiva de Portugal ${ }^{19}$, un luxueux album où vingt-quatre monuments de Porto, Lisbonne, Santarém et Sintra font l'objet d'analyses historiques et artistiques, accompagnées de photographies d'une ou deux façades de chaque bâtiment (épreuves sur papier albuminé).

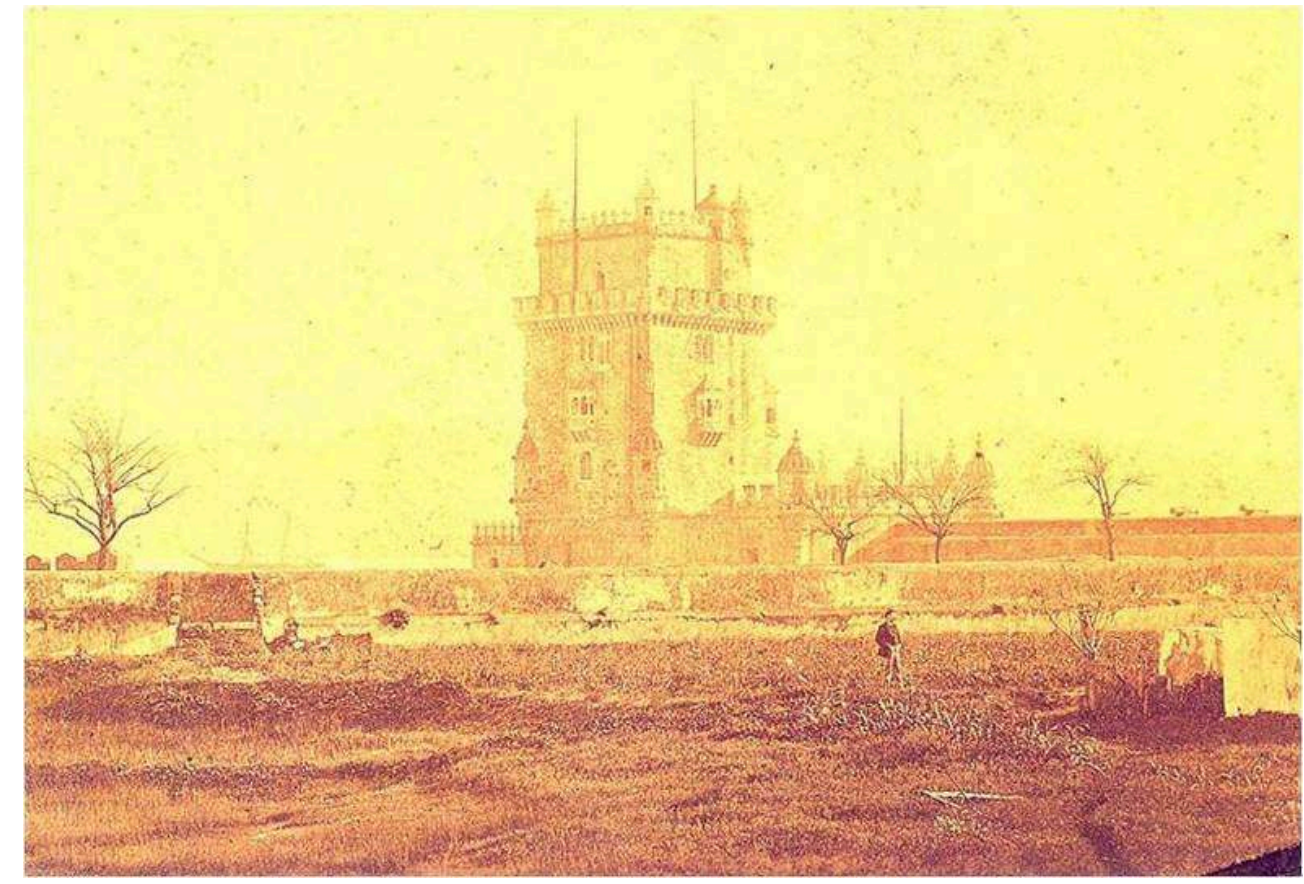

Fig. 3 : Henrique Nunes, La tour de Sao Vicente de Belém, Lisbonne, 1865, 12,8 x 18,3 cm. 
publication, qui bénéficia d'un soutien officiel ${ }^{20}$, fut pour l'architecte une consécration de ses efforts ${ }^{21}$ et lui permit de divulguer une partie de son travail lors de sa nomination en $1858^{22}$.

Possidónio da Silva souhaitait sans doute rentabiliser son travail des années précédentes, il est néanmoins curieux qu'il abandonne l'idée de publier ses dessins, pour faire le choix de la photographie. Certains auteurs attribuent ces photographies qui présentent des petites variantes dans les différents exemplaires du magazine - à Possidónio da Silva, tandis que Paulo Baptista ${ }^{23}$, sans éliminer cette hypothèse, attire l'attention sur le fait qu'une telle pratique n'était pas habituelle chez l'architecte, généralement accompagné d'un photographe, comme ce fut le cas à Caminha. Le même auteur suggère le nom d'Henrique Nunes, qui réalisa plus tard le travail photographique du Boletim da Associação dos Arquitectos Civis Portugueses [Bulletin de l'Association des Architectes civils portugais], dirigé par Possidónio da Silva.

À ce propos, il faut noter que le magazine de l'Association des Architectes (puis des Archéologues) portugais, l'Archive de l'Architecture civile, utilisa la photographie comme moyen d'illustration dès sa première édition (1865-1867). Effectivement, des photographies de bâtiments neufs, de maquettes et de vues de monuments furent publiées en nombre croissant, mais, autant que nous avons pu le constater, il s'agit surtout d'une fonction documentaire, limitée à l'illustration.

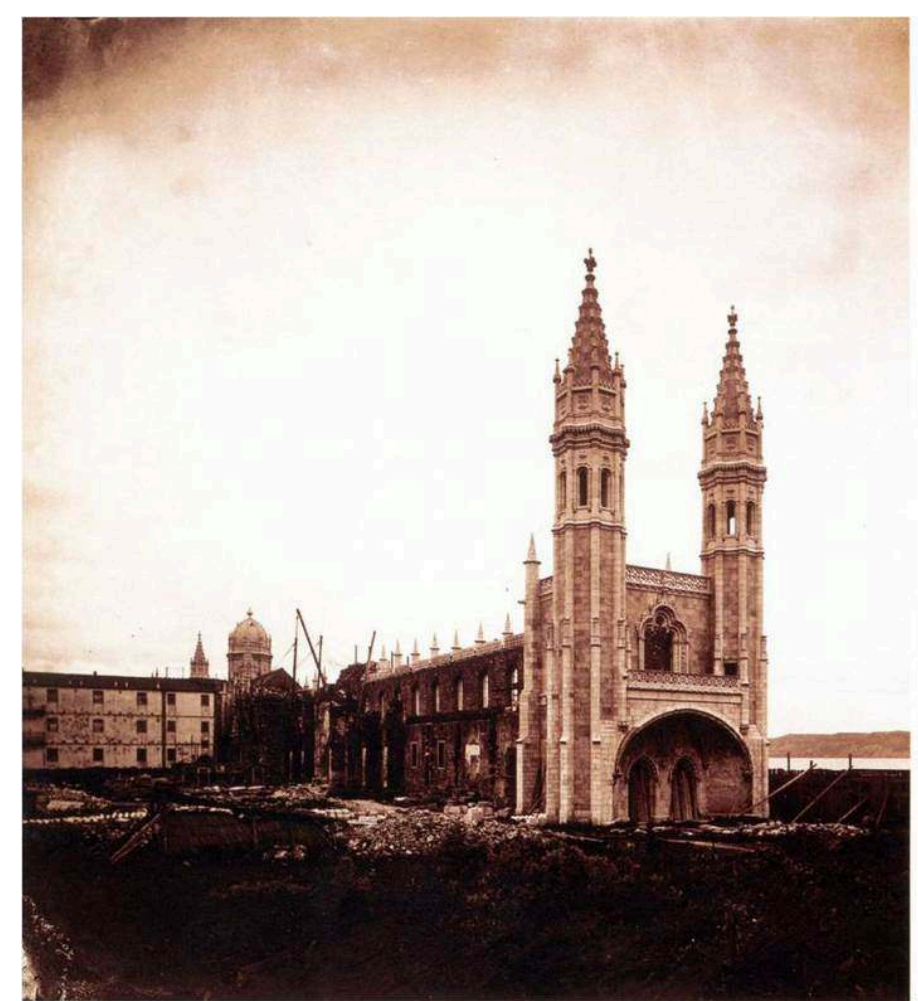

Fig. 4 : Carlos Relvas, Monastère des hiéronymites à Belém, Lisbonne, 1868-70, collodion, tirage contemporain.

19 À partir des années 1860, la présence de la photographie dans la presse périodique est plus importante, soit sur le modèle de la Revista Pittoresca, de Possidónio da Silva, soit par le biais de gravures réalisées à partir de photographies, ce qui permettait une certaine précision. C'est le cas du Panorâma Fotográfico de Portugal (1869-1974), où les textes, avec une nouvelle rigueur, étaient accompagnés d'épreuves de plusieurs 
photographes, parmi lesquels Carlos Relvas, ou du magazine 0 Ocidente (1878-1914) qui, dans les années 1870, publia fréquemment des gravures à partir des photographies du même auteur.

Cependant, il faut être prudent avec les gravures publiées, qui sont parfois des reproductions de reproductions, devenues des «images peu fidèles ou même différentes du modèle ${ }^{24} »$, donc d'une valeur documentaire douteuse.

D'autres photographies publiées sont plus crédibles, comme celles qui montrent le chantier de restauration du monastère des hiéronymites ou la construction du palais de Cristal de Porto. Construit en 1861 pour accueillir la première Exposition internationale dans la péninsule ibérique, il fut conçu par l'architecte Thomas Dillen Jones et par l'ingénieur W. Shields, s'inspirant du palais de Cristal de Londres de Joseph Paxton, érigé en 1851. L'importance de son édification pour la ville a incité des photographes professionnels, recrutés par une élite consciente du progrès technique représenté par la photographie, à documenter le chantier de construction par des épreuves sur papier albuminé et par des vues stéréoscopiques ${ }^{25}$. Dans l'Exposition internationale de 1865, il y avait une section consacrée à la photographie. Quelques années plus tard, en 1886, fut organisée dans le même bâtiment l'« Exposition de photographie de Porto ", signe de l'importance qu'avait désormais la photographie au plan national comme au plan international.

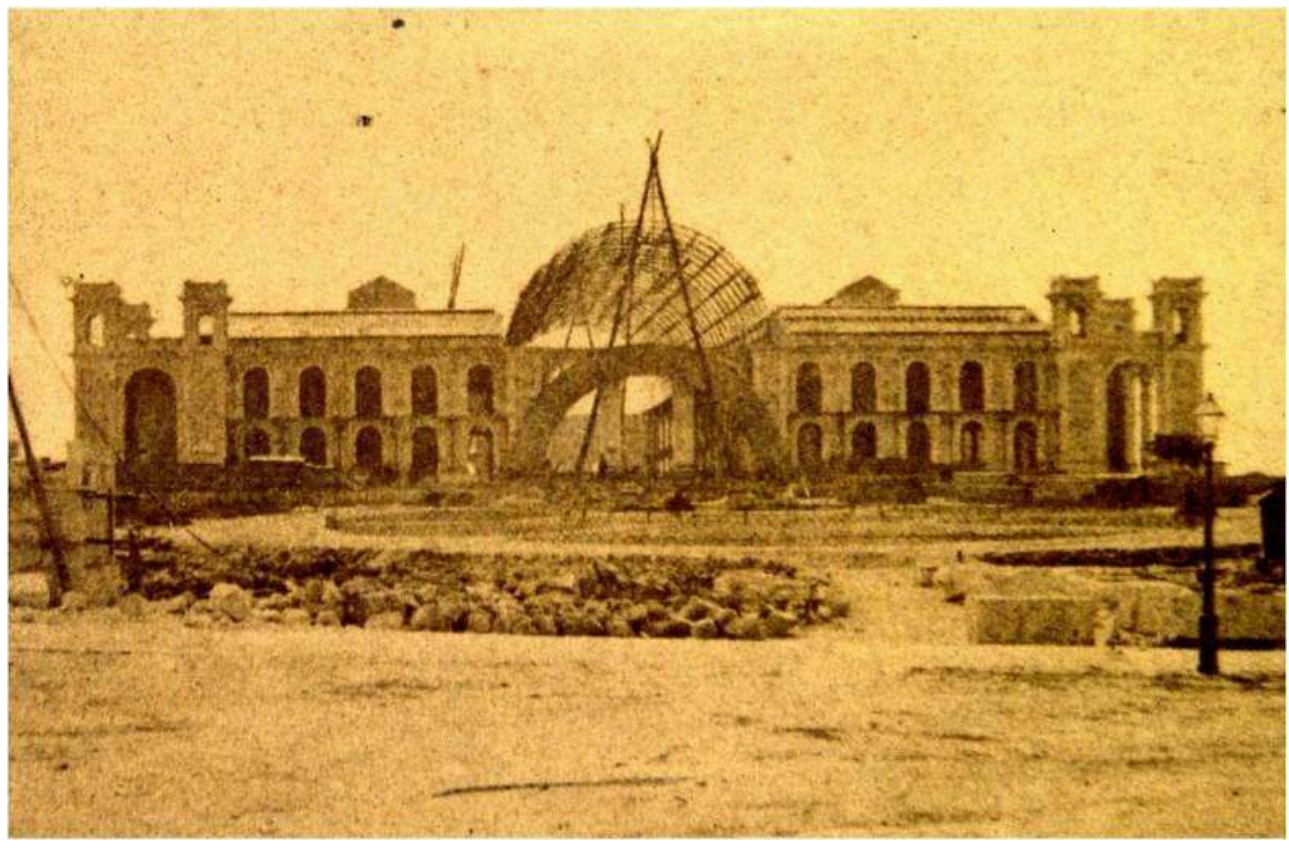

Fig. 5 : Construction du Palais de Cristal de Porto, Arquivo Histórico Municipal do Porto.

Le grand promoteur de cette exposition était le magazine de Porto Fotografia Moderna, lancé par un groupe de photographes amateurs conduit par Ildefonso Correia et soutenu par Carlos Relvas. Ce magazine, publié entre 1884 et 1885, a préparé le public à une meilleure considération de la photographie et à l'idée de son acceptation comme art. L'implication de Carlos Relvas dans ce projet est très compréhensible. Photographe amateur, il était une figure majeure de la photographie portugaise à qui l'on doit plusieurs reportages photographiques et la valeur de son travail était déjà largement reconnue en 1880 lorsqu'il fut désigné par le comité afin de « recueillir des pièces d'art portugais pour les envoyer à l'exposition du South Kensington Museum ${ }^{26}$ ", qui avait 
lieu cette année-là et, en même temps, pour rédiger un rapport sur la valeur patrimoniale des « immeubles et des objets d'art des couvents ", qu'on voulait vendre, en vue de leur sauvegarde ${ }^{27}$.

Le comité était composé d'un écrivain, Rangel de Lima, d'un architecte, Alfredo de Andrade et d'un photographe, Carlos Relvas, ce qui traduit clairement l'importance que l'on accordait à l'enregistrement photographique dans cette entreprise.

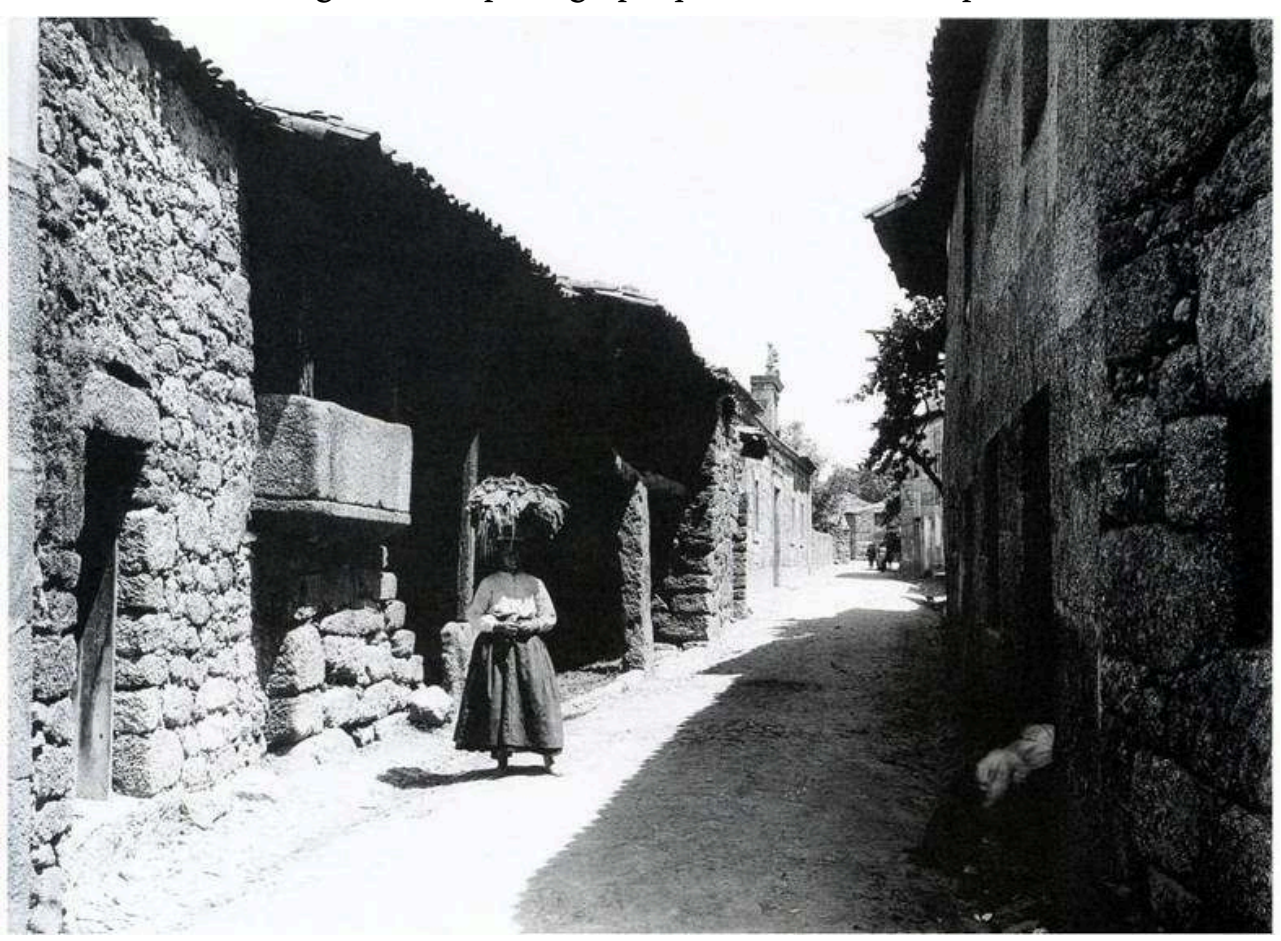

Fig. 6 : Carlos Relvas, Village, Portugal, 1880-90, négatif au gélatino-bromure d'argent, tirage contemporain.

En plus de ses épreuves, Carlos Relvas nous a laissé l'atelier de photographie qu'il avait construit lui-même en 1876, témoignage unique au monde ${ }^{28}$. Construit dans les jardins de sa maison d'Outeiro, avec pour fonction exclusive celle de studio photographique, ce bâtiment remplaçait son premier atelier, où il avait commencé à utiliser de grandes surfaces vitrées qui, combinées avec des rideaux, lui permettaient de contrôler la lumière. Ce bâtiment est l'heureux résultat d'un dialogue entre Carlos Relvas et l'architecte Henrique Carlos Afonso. Sa construction fut même commentée dans la presse de l'époque, ayant provoqué un intérêt et une curiosité autant pour le bâtiment que pour sa fonction ${ }^{29}$. 


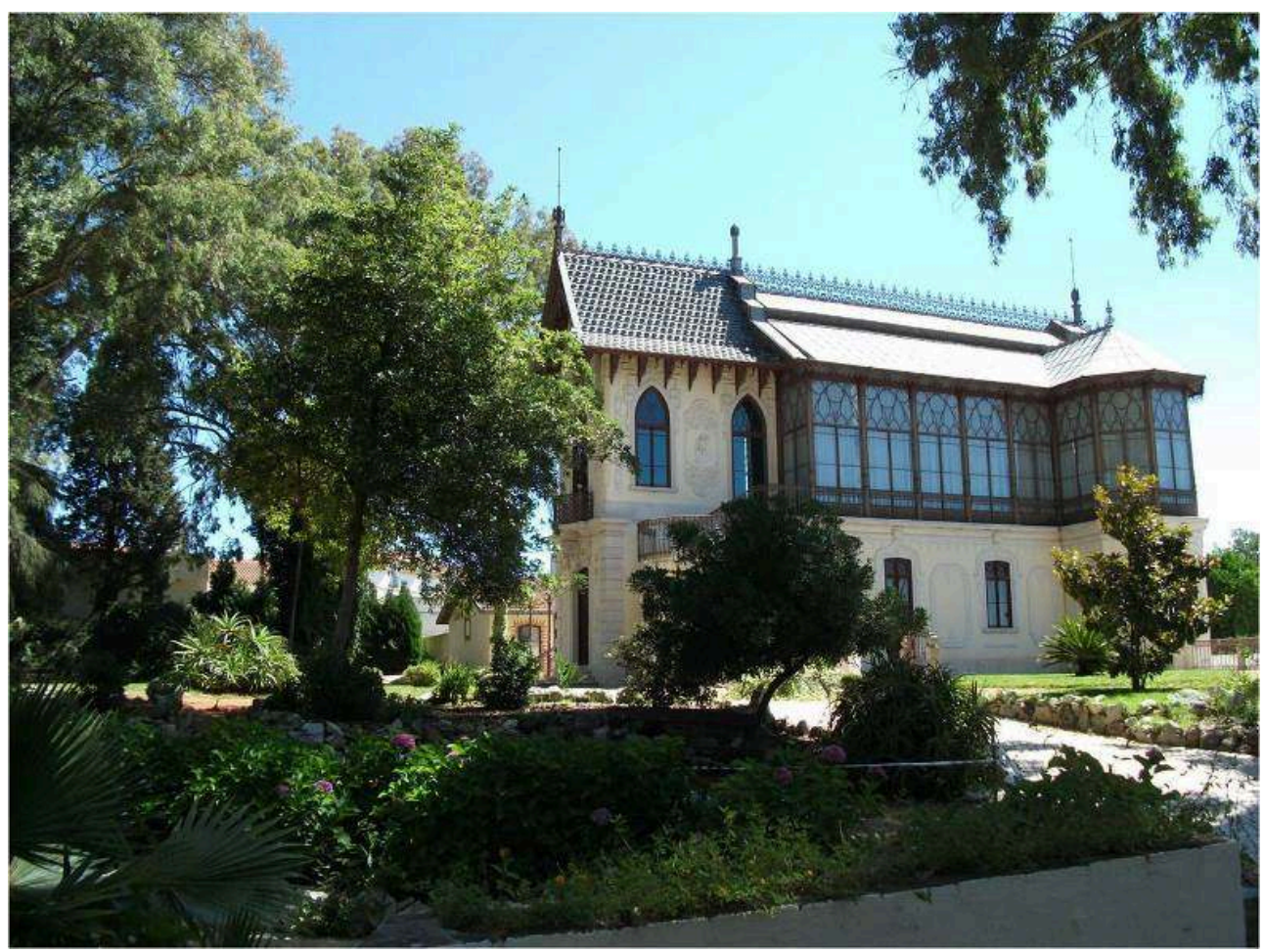

Fig. 7 : Alexandra Cardoso, Studio photographique de Carlos Relvas, projet de Henrique Carlos Afonso, Outeiro, Portugal, 1870.

Même si ce n'est pas le sujet de cet article, nous ne pouvons oublier de noter la grande qualité de la photographie d'architecture produite à partir des années 1930; la photographie intéresse de plus en plus les architectes, comme clients aussi bien que comme auteurs.

L'un des exemples les plus remarquables est celui du travail du photographe Teófilo Rego (1914-1993) produit pour l'architecte João Andresen (1920-1967) en 1955, que Miguel Moreira Pinto vient d'étudier pour le projet «Fotografia, Arquitectura Moderna e a "Escola do Porto": Interpretações em torno do arquivo Teófilo Rego $^{30}$ »[Photographie, architecture moderne et «École de Porto»: interprétations autour des archives Teófilo Rego]. 


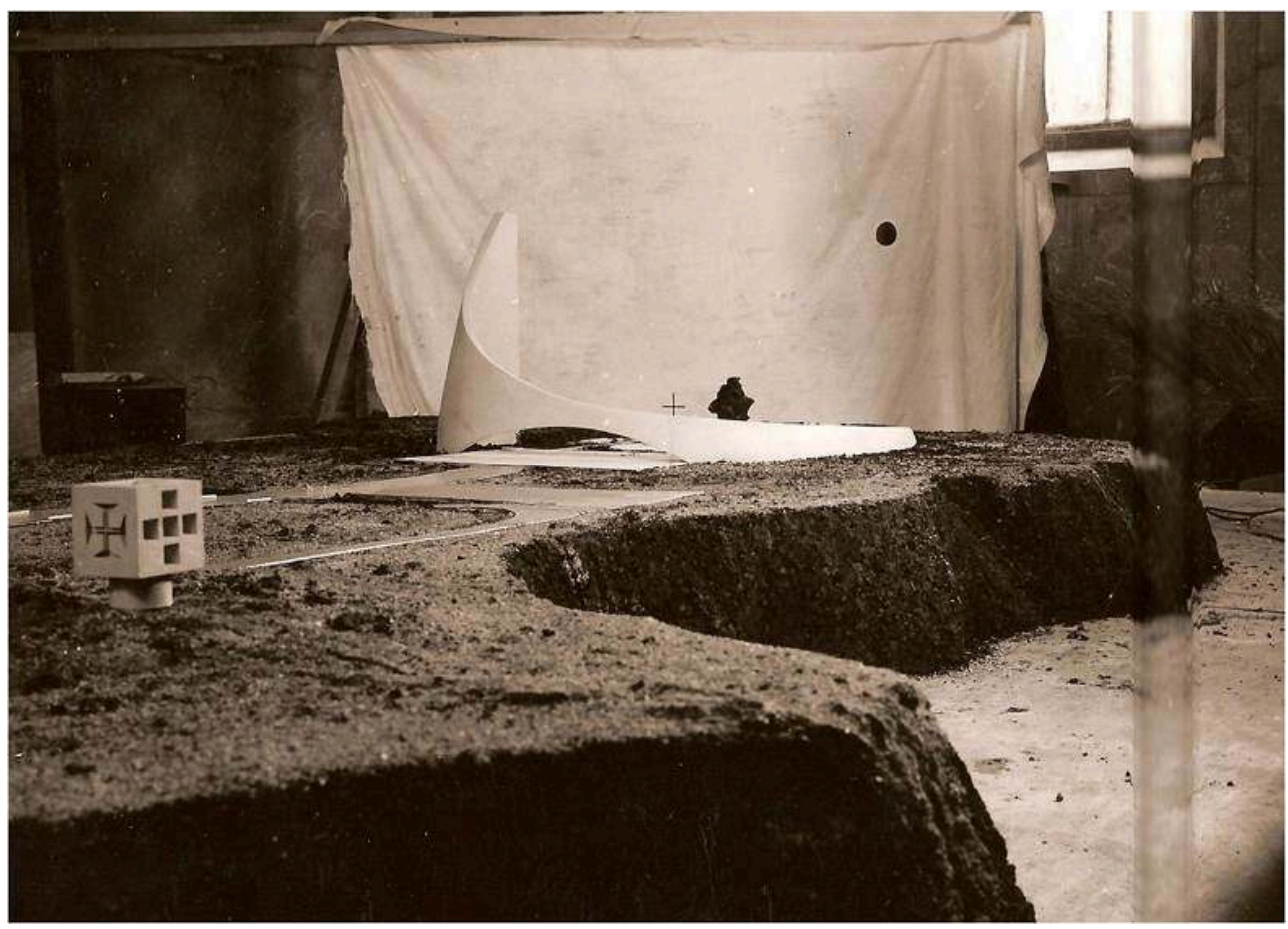

Fig. 8 : Theófilo Rego, Projet de l'architecte João Andresen, Mar Novo, 1955, tirage argentique.

27 En effet, «la surprise et l'admiration que le projet Mar Novo [Nouvelle Mer] a provoquées à son époque ${ }^{31}$ " (un projet non réalisé), n'auraient jamais pu être comprises sans «l'enregistrement photographique de la maquette (et la manipulation des images), réalisée par Teófilo Rego ", et les motivations de l'architecture moderne portugaise des années $1950^{32}$.

Pour conclure, nous aimerions encore noter que l'inventaire de l'architecture populaire portugaise ${ }^{33}$, réalisé au milieu des années 1950 par les «architectes modernes » et qui se caractérise par la place importante de la photographie, a lui aussi joué un rôle décisif dans l'évolution de l'architecture portugaise dans la deuxième moitié du $\mathrm{Xx}^{\mathrm{e}}$ siècle.

Ce travail a été financé par des fonds nationaux, par la voie de la Fundação para a Ciência e Tecnologia (FCT) dans le cadre du projet stratégique PEst-OE/EAT/ UI4041/2011. Il a aussi été financé par PIDDAC, le Fonds européen de développement régional (FEDER) et par COMPETE - Programme opérationnel pour les facteurs de compétitivité (POFC) via le projet «Photographie, architecture moderne et "École de Porto" : Interprétations autour des archives Teófilo Rego " (PTDC/ATP-AQI/4805/2012 ; FCOMP-01-0124-FEDER-028054).

\section{NOTES}

1. Augusto da Silva Carvalho, «Comemorações do Centenário da Fotografia », dans Memórias da Academia das Ciências de Lisboa, t. III, Lisbonne, 1940.

2. Voir António Sena, Uma História da Fotografia. Portugal 1839 a 1991,Lisbonne, Comissariado para a Europália 91/Imprensa Nacional Casa da Moeda, 1991, p. 8. 
3. Joaquim Viera, «Frederick W. Flower, um fotógrafo calotipista em Portugal », Colóquio Artes, n ${ }^{\circ}$ 51, déc. 1981 ; António Pedro Vicente, Carlos Relvas. Contribuição para a História da Fotografia em Portugal no séc. XIX,Lisbonne, Imprensa Nacional Casa da Moeda, 1984 ; José Pedro de Aboim Borges, Joshua Benoliel: o rei dos fotógrafos, dissertação de mestrado, Lisbonne, Nouvelle université de Lisbonne, 1984.

4. Paulo Artur Ribeiro, A Casa Biel e as suas edições fotográficas no Portugal de Oitocentos, Lisbonne, Edições Colibri/Universidade Nova de Lisboa, 2010, p. 18-22.

5. Sena 1991, p.6. Avec cet ouvrage, l'auteur cherche à esquisser un corpus en proposant une synthèse, malgré tout provisoire, de références jusque-là éparses.

6. Idem, p. 7.

7. Maria do Carmo Serén, «A Fotografia em Portugal », dans Arte Portuguesa da Pré-História ao Século XX, s. 1., Porto, Fubu Editores, 2009.

8. On peut ajouter aux travaux déjà cités de Maria do Carmo Serén ceux de Teresa Siza. Parmi les travaux universitaires, on pense notamment aux thèses réalisées en histoire de l'art contemporain à la faculté des sciences sociales et humaines de la Nouvelle université de Lisbonne: José Pedro de Aboim Borges, Joshua o rei dos fotógrafos (1984); Paulo Artur Ribeiro Baptista, A Casa Biel e as suas edições fotográficas no Portugal de Oitocentos (1994) ; Filipe Figueiredo, Nacionalismo e Picturialismo na Fotografia Portuguesa na Primeira Metade do Século xx: o caso exemplar de Domingos Alvão (2000) ; Maria Emília Tavares, João Martins (1898-1972): Imagens de um tempo "desolador descritivo" (2000). En ce qui concerne les sites internet, voir notamment le blog Arte photographica, de Sérgio B. Gomes, ou le blog d'Alexandre Pomar.

9. Serén 2009, p. 11.

10. Voir sur ce sujet, Michael Gray (dir.), Frederick William Flower. Um pioneiro da Fotografia Portuguesa, cat. exp., Lisbonne, musée du Chiado, 1994, Lisbonne, Electa/Museu do Chiado, 1994.

11. Voir à ce sujet Baptista 2010.

12. Costa Goodolphim, Biografia do sócio fundador, architecto Joaquim Possidónio Narciso da Silva lida em sessão solene de 17 de Junho de 1894, Lisbonne, Typ. Universal, 1894, p. 23.

13. José Augusto França, A Arte em Portugal no sec. XIX. vol. I, Lisbonne, Livraria Bertrand, 1967, p. 383 ; Boletim da Associação dos Architectos Civis e Archeologos Portugueses, série 2, t. I, 1875, p. 60.

14. Fondé en 1846, le Grémio Literário est « une sorte d'Académie des Beaux-Arts, alternative à l'officielle, avec la Nouvelle École d'Architecture », créée par Possidónio da Silva. Ses œuvres y étaient exposées : celles produites lors de son séjour à Paris, comme les modèles de bâtiments et d'antiquités recueillies au cours de son séjour en Italie. Bien que le magazine Revista Universal Lisbonense ait prédit le succès de cette école, cela ne s'est pas réalisé. Voir Raquel Henriques da Silva, Lisboa Romântica. Urbanismo e Arquitectura, 1777-1874, vol. I, dissertação de doutoramento, Lisbonne, Nouvelle université de Lisbonne, 1997, p. 360.

15. Selon França 1967, p. 320.

16. Júlio de Castilho, Elogio Histórico do architecto Joaquim Possidónio Narciso da Silva, proferido em sessão solene da Real Associação dos Architectos Civis e Archeologos Portugueses em 28 de Março de 1897,Lisbonne, Typ. da Real Academia das Sciencias, 1897, p. 26.

17. Possidónio da Silva, Relatório da Commissão dos Monumentos Nacionaes Apresentado ao Illustrissimo e Excelentissimo Senhor Ministro das Obras Publicas, Commercio e Industria pelo Presidente da Referida Commissão em 1884,Lisbonne, Imprensa Nacional, 1894,p. 20 et 26.

18. Ibid., p. 10, 16 et 11.

19. Le matériel ici publié circulait déjà sous la forme d'un prospectus de 1861. Voir Baptista 2010, p. 92.

20. Ibid.

21. Jorge Custodio, «Revista Pittoresca e Descriptiva de Portugal » dans Dar Futuro ao Passado. Catálogo das obras expostas, Lisbonne, Instituto Português do Património Arquitectónico e Arqueológico, 1993. 
22. Baptista 2010.

23. Ibid.

24. Lúcia Cardoso Rosas, Monumentos Pátrios. A arquitectura religiosa medieval - património e restauro (1835-1928), dissertação de doutoramento, Porto, Université de Porto, 1995, vol. I, p. 90.

25. Comme par exemple Miguel Novais, Henrique Nunes et Manuel José de Sousa Ferreira. Voir Serén 2009, p.17.

26. Comité d'abord composé du peintre Ferreira Chaves et de Rangel de Lima. C'est le premier qui aurait eu l'idée d'inviter le photographe Carlos Relvas à rejoindre l'équipe. Le nom d'Alfredo Andrade était apparu suite à l'impossibilité pour Chaves Ferreira de faire les trajets nécessaires à cette tâche. Voir Lucília Verdelho da cosTA, Alfredo de Andrade (1839-1915) Da Pintura à Invenção do Património,Lisbonne, Vega, 1997, p. 237, n. 1.

27. Ruy de Andrade, Vida de um artista português no século XIX em Itália,Lisbonne, Escolas Profissionais Salesianas - Of. S. José, 1996, p. 125.

28. Voir http://www.casarelvas.com/site/pt/index.php.

29. À titre de curiosité, il faut remarquer que les laboratoires se situaient au rez-de-chaussée, où il y avait aussi une réception. Au premier étage se situait le studio.

30. Projet actuellement en cours dirigé par Miguel da Silva Graça au CEAAda ESAP e Casa da Imagem da Fundação Manuel Leão, Porto/Portugal, qui a reçu le financement de la FCT (PTDC/ ATP-AQI/4805/2012) pour la période 2013-2015.

31. Inês Azevedo, Alexandra Trevisan et al., «Fotografia e Arquitectura Moderna: a visão de Teófilo Rego e a Nova Monumentalidade de João Andresen », Scopio International Photography Magazine, $\mathrm{n}^{\circ}$ 2, Matosinhos, Cityscopio, 2013.

32. Texte d'application du projet «Photographie, architecture moderne et "École de Porto": interprétations autour des archives Teófilo Rego »(PTDC/ATP-AQI/4805/2012), présenté à la FCT en mai 2012.

33. Arquitectura Popular em Portugal (Lisbonne, Sindicato Nacional dos Arquitectos, 1961), $4^{\mathrm{e}}$ éd. : Lisbonne, Centro Editor Livreiro da Ordem dos Arquitectos, 2004, 2 vol. Cette enquête a fait l'objet d'un projet de recherche du CEAA : «A "Arquitectura Popular em Portugal". Uma Leitura Crítica» (FCT : PTDC/AUR-AQI/099063/2008 - COMPETE : FCOMP-01-0124-FEDER-008832), entre 2010 et 2013.

\section{AUTEURS}

\section{ALEXANDRA TREVISAN}

Escola Superior Artística do Porto - Centro de Estudos Arnaldo Araújo - FCT uID 4041

\section{MARIA HELENA MAIA}

Escola Superior Artística do Porto - Centro de Estudos Arnaldo Araújo - FCT uID 4041 
Les rêveries photographiques d'un architecte. La collection Alfred Armand au département des Estampes et de la photographie de la Bibliothèque nationale de France

\author{
Thomas Cazentre
}

Des diverses collections thématiques constituées par des particuliers et conservées au département des Estampes et de la photographie ${ }^{1}$, celle que légua en 1888 l'architecte Alfred Armand est quantitativement l'une des plus considérables. C'est aussi l'une des plus méconnues. Cette « collection de photographies formant une histoire générale de l'art ", selon les termes mêmes du testament d'Alfred Armand, entreprise considérable et pionnière en son temps, a en effet souffert du désintérêt qui a frappé toutes les collections de ce genre dans la seconde moitié du $\mathrm{xx}^{\mathrm{e}}$ siècle, lorsque la profusion de reproductions et de publications artistiques a réduit à presque rien la visée documentaire selon laquelle elles avaient été constituées. Depuis une vingtaine d'années toutefois, grâce au travail conjoint des conservateurs et des chercheurs, elle a fait l'objet d'une redécouverte, mais dans une perspective nouvelle. Car, si cette collection demeure aujourd'hui encore un ensemble passionnant, c'est désormais moins par rapport à l'histoire de l'art que par rapport à celle de la photographie, en particulier comme moyen de représentation de l'architecture. Sa richesse et son originalité se fondent en effet sur la qualité propre des images rassemblées, mais aussi sur les partis pris et les inclinations qui ont guidé ce rassemblement, non sans une certaine dimension énigmatique ; énigme où la personnalité curieuse du collectionneur a aussi sa part. 


\section{Alfred Armand (1805-1888), architecte à succès et esthète contrarié}

2 Toute collection est, en quelque manière, un portrait intime du collectionneur. À cet égard, le contraste entre la carrière publique d'Armand et ce que révèlent ses choix de collectionneur tient presque du cas d'école pour illustrer la dissociation proustienne du moi social et du moi profond.

3 Alfred Armand ${ }^{2}$ naît à Paris le 8 octobre 1805. En 1827 il entre à l'École des beaux-arts en section Architecture. Sa scolarité et ses débuts restent obscurs et il faut attendre 1835 pour que sa carrière prenne un essor décisif, dans un domaine apparemment peu prestigieux mais qui va faire sa fortune: les chemins de fer. Armand est en effet désigné architecte principal de la Compagnie du chemin de fer de Paris à SaintGermain-en-Laye, fondée par Émile Péreire, et pour laquelle il construit des embarcadères et des ouvrages d'art. À partir de ce moment, Armand devient l'architecte des frères Péreire, au service desquels il va travailler de manière quasi exclusive. Pionnier de l'architecture ferroviaire, il conçoit ainsi la première grande gare parisienne, Saint-Lazare (1841-1842), et les gares du chemin de fer du Nord (1845).

4 En 1852, toujours à l'initiative des Péreire, il se réoriente vers un nouveau type de construction : les grands hôtels. Armand importe ainsi en France le modèle américain de l'hôtel à plusieurs centaines de chambres, avec des espaces publics monumentaux. Ce fut d'abord l'Hôtel du Louvre, puis le Grand Hôtel du boulevard des Capucines, qui tous deux firent sensation. Au cours de cette même décennie, il réalise pour les Péreire l'aménagement intérieur de l'hôtel du Faubourg Saint-Honoré et le château d'Armainvilliers.

Or, en 1863, en pleine réussite, Armand met fin à sa carrière d'architecte pour se consacrer exclusivement à l'art, comme collectionneur et érudit. Cette brusque reconversion n'est pas moins intrigante par la direction qu'elle prend: en effet, alors que sa carrière d'architecte avait été nettement placée sous le signe de la modernité technique, de l'utilitarisme voire de la spéculation, sa seconde vie de collectionneur est dédiée à l'art ancien, sous ses formes les plus modestes; comme si derrière le succès professionnel s'était caché un esthète contrarié. Il entreprend ainsi une collection de dessins sous l'impulsion de son ami le graveur Henriquel-Dupont ; mais c'est à un autre objet de collection, et surtout d'étude, qu'il dédie les quinze dernières années de sa vie : les médailles italiennes de la Renaissance, auxquelles il consacre un ouvrage de référence, Les Médailleurs italiens des XVe et XVI siècles (1879-1887).

Le peu d'informations que l'on a sur sa vie privée donne toutefois une dernière dimension particulière à sa vocation de collectionneur : il avait épousé en 1843 une jeune fille qui mourut dix-huit mois plus tard, sans qu'ils aient eu d'enfant; il ne se remaria jamais. L'essentiel de son œuvre architecturale a disparu, à l'exception du Grand Hôtel ; et il avait eu peu avant sa mort la douleur de voir détruire ou dénaturer ses deux réalisations les plus emblématiques : la gare Saint-Lazare, démolie pour faire place au bâtiment actuel [fig. 1], et l'Hôtel du Louvre, remanié de fond en comble pour être transformé en magasin (c'est l'actuel Louvre des antiquaires). Son seul héritage est ainsi constitué par ses collections, dont il prit soin à la fin de sa vie d'assurer la pérennité. Il transmit ses dessins à son ami Prosper Valton, qui, à sa propre mort en 1907, les légua à l'École des beaux-arts ${ }^{3}$; les médailles, par l'intermédiaire du même 
Valton, aboutirent au Cabinet des médailles de la Bibliothèque Nationale; quant aux photographies, elles furent léguées dès 1888 au Cabinet des estampes.

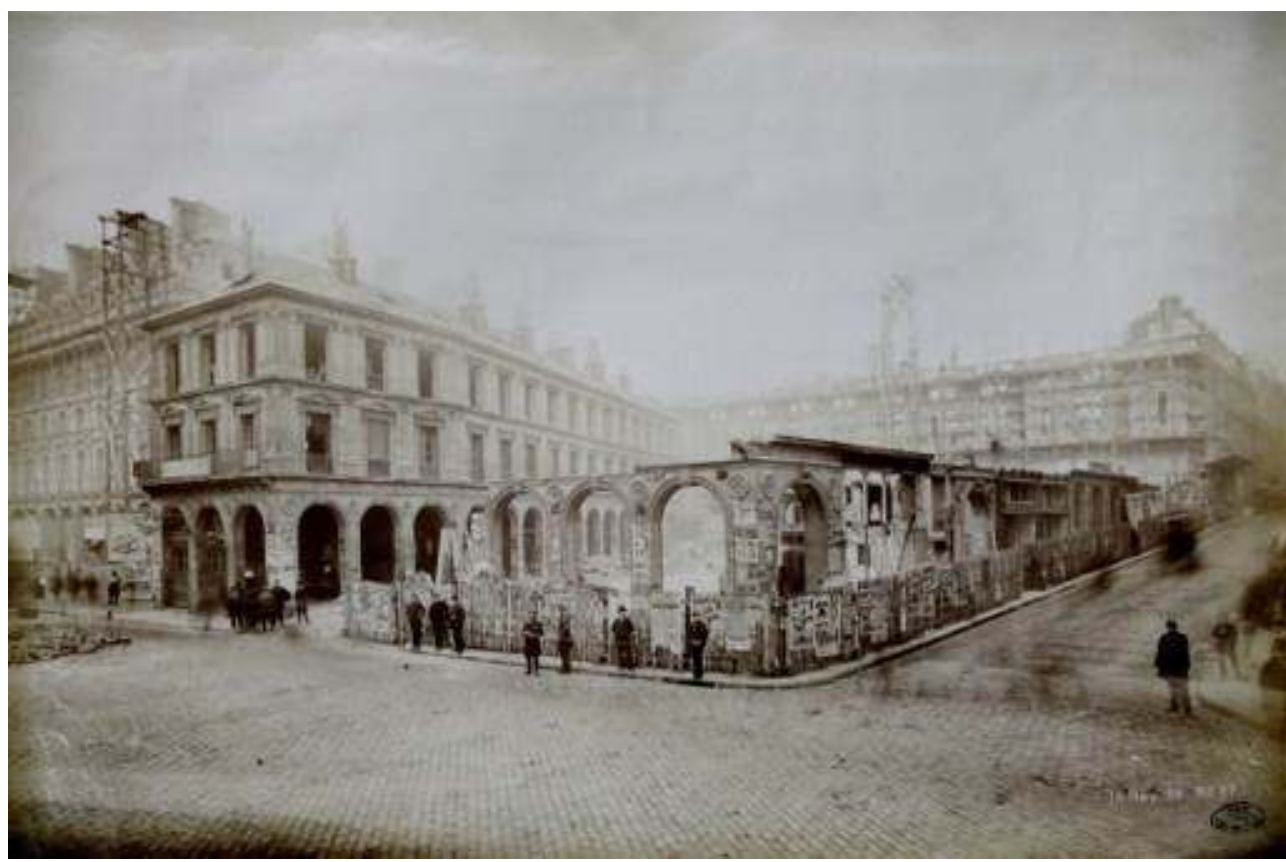

Fig. 1 : Anonyme, Démolition de l'ancienne gare Saint-Lazare à Paris, 16 novembre 1888, tirage sur papier albuminé, Paris, Bibliothèque nationale de France, département des Estampes et de la photographie, VA-282 (A, 1)-FOL.

\section{La collection de photographies : composition et constitution}

7 Cette collection iconographique sur l'histoire générale des arts, de l'Antiquité au XIX $X^{e}$ siècle, comptait initialement 19410 pièces réparties en quelque 200 portefeuilles. À la suite du legs, l'intégralité de la collection a été montée en à peu près autant de volumes reliés (230 au total) ${ }^{4}$, en respectant scrupuleusement le classement et l'ordre d'origine. Les documents furent contrecollés selon la méthode alors en usage, mais en reportant systématiquement les indications manuscrites d'Armand au verso. Un inventaire pièce à pièce fut établi par le bibliothécaire François Bourgoin ${ }^{5}$. Dans la préface de cet inventaire, le conservateur Georges Duplessis atteste l'intérêt exceptionnel de cette collection et explique le traitement particulièrement respectueux dont elle a fait l'objet, à l'instar d'autres collections léguées au Cabinet des estampes à la même époque (collection Hennin sur l'histoire de France, collection Fleury sur le département de l'Aisne...).

Les tomes 1 à 36 sont consacrés à l'Antiquité (les tomes 1 et 2 rassemblent l'Orient ancien, l'Égypte et l'Asie mineure ; Grèce et Italie occupent les 34 autres). Les tomes 37 à 158 portent sur l'histoire de la peinture (t. 37-114 : école italienne; t. 115-118 : école espagnole; t. 119-136 : école française; t. 137-158 : écoles du nord) ; les tomes 159 à 219, sur l'architecture et la sculpture (t. 159-160: domaines extra-européens : Mexique, Asie, Égypte et Turquie ; t. 161-194: Italie ; t. 195-211 : France ; t. 212-213 : Europe centrale et septentrionale; t. 214-219: péninsule ibérique). Enfin les onze derniers tomes 
rassemblent une iconographie diverse (t. 220-221: vues d'Italie et de France; t. 222-223 : gravure; t. 224-230 : objets d'art).

9 Cette brève présentation suffit à faire apparaître la dominante très nette, dans les domaines traités, de l'Italie, de l'Antiquité classique et de l'art français, en dépit d'un réel souci d'universalisme. Historiquement, la place prépondérante va à l'art du Moyen Âge et de la Renaissance ; les $\mathrm{XVII}^{\mathrm{e}}$, XVIII ${ }^{\mathrm{e}}$ et $\mathrm{XIX}^{\mathrm{e}}$ siècles sont traités de manière beaucoup plus expéditive ; ainsi, dans la série sur l'architecture et la sculpture italiennes, un seul et dernier tome sur 34 couvre l'intégralité de la période 1640-1880...

10 La collection est explicitement désignée comme " photographique » dans le testament d'Armand, même si la photographie est loin d'en être le support unique. L'étude de la répartition entre photographies et estampes dans la représentation des monuments justifierait une étude en soi. Il semble bien qu'Armand ait eu pour principe de privilégier la photographie, les autres techniques n'intervenant qu'en appoint ou en complément : soit en l'absence de documents photographiques, soit pour des types de représentation spécifiques (plans, coupes, détails, restitutions) de certains sites et monuments pour lesquels Armand a souhaité constituer une couverture documentaire plus exhaustive et académique (Pompéi, Acropole d'Athènes). Cette prédilection massive et précoce pour la photographie suffirait en elle-même à marquer l'originalité de la collection.

11 Pour les domaines non occidentaux, la présence ou l'absence de certains pays paraît ainsi s'expliquer assez clairement par l'existence ou non d'une documentation photographique, ou plus exactement par la possibilité ou non de se procurer des photographies en France : ainsi le Mexique figure-t-il grâce à Désiré Charnay, l'Inde grâce à Alexis de Lagrange et Louis Rousselet; a contrario, ni la Chine, ni l'Afrique du Nord, ni même la Russie ne sont représentées. Il est moins facile d'expliquer la part très minoritaire des photographies dans la représentation de l'architecture française, surtout en comparaison avec l'Italie. Une hypothèse plausible serait qu'Armand a accumulé son iconographie sur la France à une époque où la couverture photographique du territoire était encore très incomplète, à quelques notables exceptions près qui apparaissent d'ailleurs dans la collection (Baldus, Bisson, Marville, Roman, Lancelot...) ; et qu'à partir des années 1860 il s'est surtout intéressé à l'Italie, négligeant les gros corpus français de photographies d'architecture qui se constituaient alors. Mais il n'est pas absurde de penser que ces répartitions divergentes trahissent aussi une différence de regard et de rapport intime d'Armand envers les arts français et italiens; plus professionnel et archéologique dans le premier cas, plus contemplatif dans le second.

12 Faute d'informations de première main, on doit se livrer à de semblables conjectures pour établir la manière dont Armand a constitué cette collection. D’un point de vue chronologique, si l'on en croit Georges Duplessis, c'est essentiellement dans la seconde moitié de sa vie, après 1862, qu'Armand aurait rassemblé ces documents, et ce jusqu'à sa mort; mais on trouve dans la collection de nombreuses photographies datant des années 1850 , et même quelques incunables de l'édition photographique française, telles les vues d'Inde par Lagrange, d'Égypte par Maxime Du Camp et de Belgique par Eugène Desplanques, tirées par Blanquart-Evrard respectivement en 1851, 1852 et 1854. On pourrait encore citer d'autres noms marquants de la photographie des années 1850 dont Armand n'a pu acquérir les tirages que sur le moment, comme Gabriel de Rumine ou Eugène Piot ; c'est encore plus vrai d'un Édouard de Campigneulles, dont les vues 
d'Orient n'ont été exposées qu'une seule fois, en 1859, et ont dû attendre 2005 pour être identifiées ${ }^{6}$.

Nulle trace en revanche de photographes qui ont massivement produit des vues d'architecture touristiques à partir des années 1870 : Neurdein ou Mieusement pour la France, James Valentine ou George Washington Wilson pour la Grande-Bretagne, Bonfils pour le Proche-Orient... Les ateliers comparables en Italie (Alinari, Brogi, Naya) et en Espagne (Laurent) sont, eux, massivement présents dans la collection, ce qui tendrait à confirmer l'hypothèse d'une constitution en deux temps de la collection selon les pays représentés : les photographies ont été acquises dès le début des années 1850 et jusqu'à la fin des années 1860, à l'exception de l'Espagne et de l'Italie, pour lesquelles de nombreuses images sont acquises plus tardivement. On peut d'ailleurs noter que, pour la France, l'histoire de l'architecture prend fin, dans la collection, avec le début de la IIIe République (destructions de 1871, projets de reconstruction de l'hôtel de ville de Paris et plans d'Abadie pour le Sacré-Cœur).

Les lieux de fourniture d'Armand, en dehors de France, peuvent être déterminés dans une certaine mesure. Il est plus que probable qu'il ait acquis la plupart de ses photographies d'Italie lors de ses nombreux voyages, surtout dans la seconde partie de sa vie. Si certains "gros" producteurs d'images comme Alinari étaient diffusés en France, la présence de photographes beaucoup plus locaux comme Rossetti et Limono à Brescia, Pozzi à Milan ou Paganori et Philpot à Florence, plaide pour des achats sur place. Il en est vraisemblablement de même pour l'Espagne.

Le cas est moins net pour les autres destinations européennes (Allemagne, Angleterre, Autriche) : le nombre restreint de photographies (même s'il s'explique peut-être par le moindre intérêt d'Armand pour l'architecture de ces pays) et le fait qu'on ne relève pour chaque pays qu'un ou deux noms de photographes (Jägermayer, Miethke et Wawra pour l'Autriche, Wiedmayer pour la Bavière, Thurston Thompson pour l'Angleterre) peut laisser supposer une acquisition en France, auprès d'un revendeur comme Giraudon. Enfin, en ce qui concerne l'Orient (Grèce, Turquie, Syrie, Liban, Palestine, Égypte), le caractère hétérogène et parcellaire des photographies rassemblées trahit là aussi de probables acquisitions en France au fil du temps et des occasions - ce qui implique qu'Armand ne s'est probablement jamais rendu dans ces pays, en dépit de la fascination qu'ils exerçaient sur lui. Soit il s'agit de photographies de voyageurs français qui n'ont été publiées qu'à leurs retours (Du Camp, Beaucorps, Campigneulles), soit de photographes locaux (Constantin, Robertson et Beato) dont on sait qu'ils ont été exposés à Paris ; les vues de Palestine de Frank Mason Good portent quant à elles le timbre sec d'un diffuseur parisien (Ziegler). C'est d'ailleurs dans cet ensemble «orientaliste» qu'Armand se révèle le plus nettement comme un collectionneur de photographies, plus que comme un collecteur d'iconographie.

\section{Collection d'architecte ou collection d'esthète?}

16 Si le mode de constitution de la collection Armand ne peut être cerné qu'au moyen de recoupements et de probabilités, le sens même de cette collection relève, quant à lui, de la pure interprétation, pour ne pas dire de l'extrapolation, Armand n'ayant laissé aucun témoignage, aucune explication sur ce qui l'a conduit à accumuler ces images d'art. préparatoire à un projet de livre. La vocation d'historien d'art a été très tardive chez 
Armand ; elle n'a été pour ainsi dire qu'une conséquence inattendue de son activité de collectionneur (faute d'ouvrage de référence sur les médailles italiennes de la Renaissance, il s'est employé à en produire un lui-même) et n'a porté que sur un domaine bien précis. Le soin scrupuleux et le goût de l'exhaustivité qu'il a manifestés dans cette entreprise paraissent d'ailleurs franchement incompatibles avec un projet d'histoire générale et universelle des arts.

On ne peut non plus avancer qu'Armand ait voulu, avec cette collection, rassembler une documentation pour sa propre activité d'architecte. La première raison, qui suffirait seule à démentir cette hypothèse, est le décalage chronologique entre ces deux moments de sa vie, même si nous avons vu qu'il n'était pas absolu : la grande majorité des photographies ont été acquises alors qu'Armand avait déjà mis fin à sa carrière d'architecte. Mais plus significatif, sinon plus probant, est le décalage entre l'architecture documentée dans la collection et l'œuvre d'Armand; d'un côté une œuvre inscrite dans la modernité commerciale et industrielle, de l'autre une prédilection pour l'architecture ancienne et sacrée que l'on pourrait presque qualifier de préraphaélite.

On pourrait certes pointer, dans ce que l'on peut savoir de l'œuvre architecturale d'Armand, un vocabulaire "néo-renaissant » qui pourrait éventuellement résonner avec le tropisme antiquisant et italianisant à l'œuvre dans la collection; mais il ne s'agit en fait que d'un style très commun et impersonnel de l'époque, et surtout il ne concerne que l'enveloppe esthétique de bâtiments dont la fonction et l'originalité essentielles résidaient dans leur structure interne. Il faut en outre rappeler que cette collection est conçue comme une "histoire générale de l'art ", dont l'architecture n'est qu'une composante, minoritaire et non isolée, à côté d'autres arts qu'Armand n'a jamais pratiqués; c'est donc moins la collection d'un architecte que celle d'un collectionneur, et plus encore d'un esthète, puisque l'ampleur de la matière représentée dépasse largement ses intérêts de collectionneur.

Indéniable en revanche est l'impact, dans sa démarche de collectionneur, de sa formation d'architecte aux Beaux-arts. Elle se trahit aussi bien dans les périodes et les édifices privilégiés que dans la manière de constituer, sur certains d'entre eux, des dossiers iconographiques complets mêlant plans, dessins, reconstitutions et photographies. Mais le fait même qu'Armand ait continué à rassembler de tels ensembles iconographiques, hors de toute contrainte scolaire ou professionnelle, ne manque pas d'intriguer. Surtout, trois exemples au moins témoignent du caractère profondément anti-utilitaire de ces « dossiers » : parmi les ensembles monumentaux les plus exhaustivement couverts dans la collection, on trouve en effet Pompéi (cas tout à fait exceptionnel puisque, avec les peintures d'Herculanum, la ville exhumée occupe treize tomes entiers, soit une collection propre à l'intérieur de la collection), l'Alhambra de Grenade (presque un tome complet) et, dans une moindre mesure, la Cité de Carcassonne, ou plus exactement sa restauration par Viollet-le-Duc (43 images, plus que pour aucun autre monument français). Autrement dit, tout ce qui, dans l'espace européen, s'éloigne le plus, dans le temps et dans l'esprit, de l'architecture fonctionnelle et bourgeoise à laquelle Armand a consacré l'essentiel de son activité ; trois lieux appartenant à un passé ou à un ailleurs absolus, et dont la préservation ou la réinvention tiennent de l'exception voire du miracle; miracle que les images, et les photographies en particulier, viennent en quelque sorte fixer, attester et prolonger. 
21 Tout tend ainsi à prouver qu'Armand n'a pas accumulé cette iconographie à des fins utilitaires, mais bien pour constituer une évocation visuelle aussi complète de monuments qui le fascinent; ce ne sont pas pour lui des documents de travail, mais bien des supports de rêverie, de délectation. L'architecture de la collection photographique apparaît comme l'antithèse voire l'échappatoire, la consolation d'une certaine trivialité architecturale à laquelle il a dû se soumettre dans sa carrière; antithèse qu'il ne faut toutefois pas pousser trop loin, car il faut noter qu'Armand a inclus, modestement mais à leur place, ses propres réalisations dans cette histoire, comme pour une réconciliation des deux moitiés de sa vie.

\section{Avènement du regard photographique sur l'art et le monde}

C'est à l'aune du caractère fondamentalement esthétisant de cette collection qu'il faut reconsidérer le choix délibéré de la photographie comme support privilégié, alors qu'Armand appartenait à une génération qui avait été formée à la représentation architecturale avant l'invention de la photographie.

Remarquons d'abord, même si cela va de soi aujourd'hui, que le statut de la photographie n'est pas le même lorsqu'il s'agit de représenter la peinture d'une part et l'architecture d'autre part (sculptures et objets d'art constituant, du fait de leur tridimensionnalité, des objets photographiques hybrides, sur lesquels notre regard a radicalement évolué depuis le XIx siècle). Dans le premier cas, c'est l'exactitude de la reproduction, supérieure à n'importe quelle copie dessinée ou gravée, qu'Armand a recherchée. Dans le second, on constate au contraire qu'Armand a retenu, même minoritairement, des photographies dont la valeur documentaire est faible (du fait de la distance au monument, de l'angle choisi, de l'environnement qui fait écran ou distraction...), mais qui valent par leur puissance d'évocation.

Certaines photographies ont manifestement été choisies pour l'information qu'elles donnent non sur l'architecture elle-même, mais sur le contexte, l'insertion du monument dans son environnement. Ainsi, dans le tome consacré aux monuments grecs de Sicile, trois photographies successives (sans doute de Paul Berthier) « introduisent » le temple de Ségeste en un effet de zoom qui mime aussi la progression du voyageur [fig. 2 et 3 ]: d'abord une vue d'ensemble de la vallée sauvage où s'élève aujourd'hui le temple, et où il n'est qu'un point minuscule; puis un cadre plus rapproché où le temple apparaît, central dans sa splendide solitude; enfin une vue frontale de la façade, qui précède des gravures du temple reconstitué. 


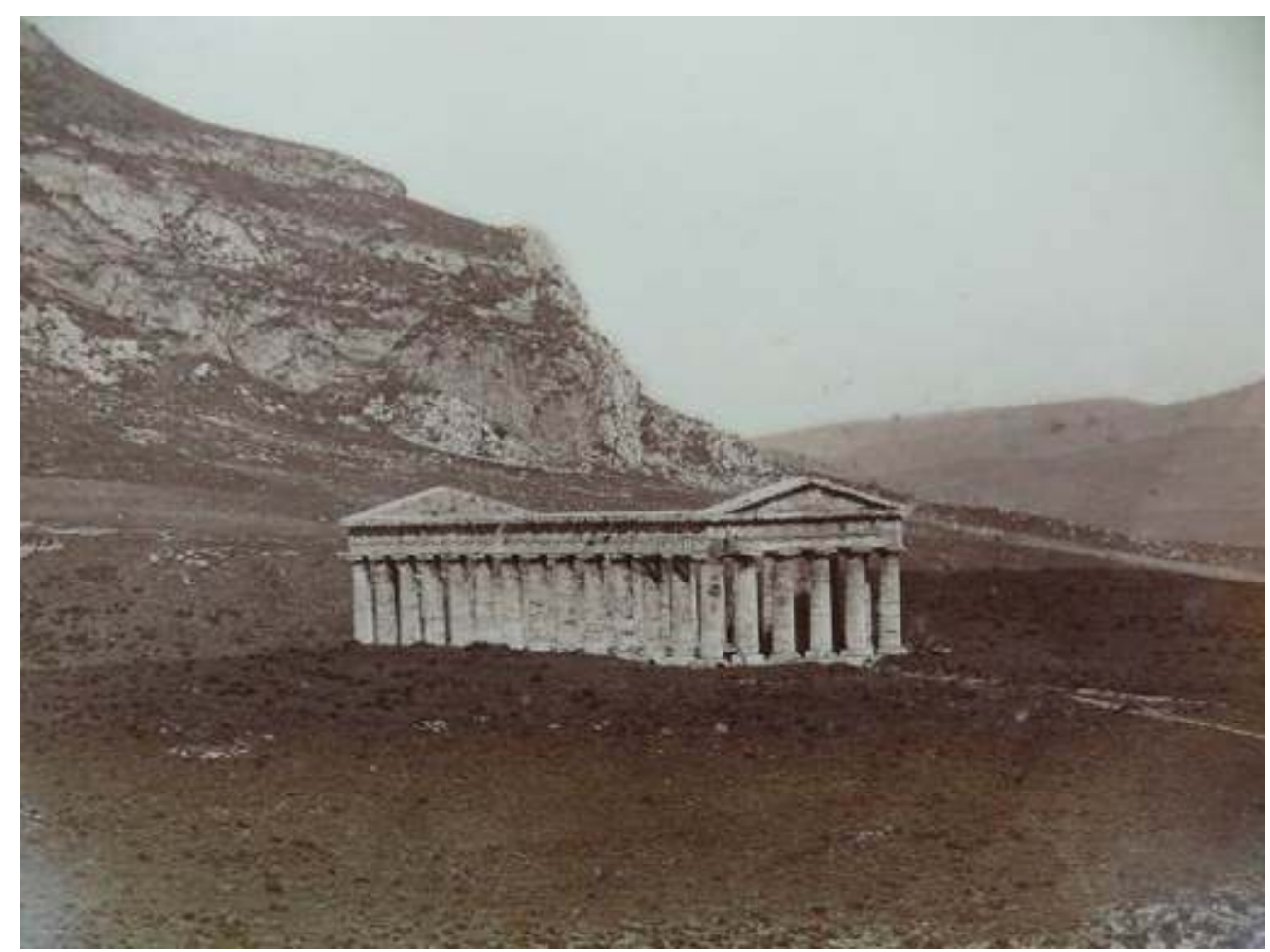

Fig. 2 : Paul Berthier [attr.], Temple de Ségeste, Sicile, vue distante, vers 1865, tirage sur papier albuminé, Paris, Bibliothèque nationale de France, département des Estampes et de la photographie, collection A. Armand, AD-34 (A, 7)-FOL.

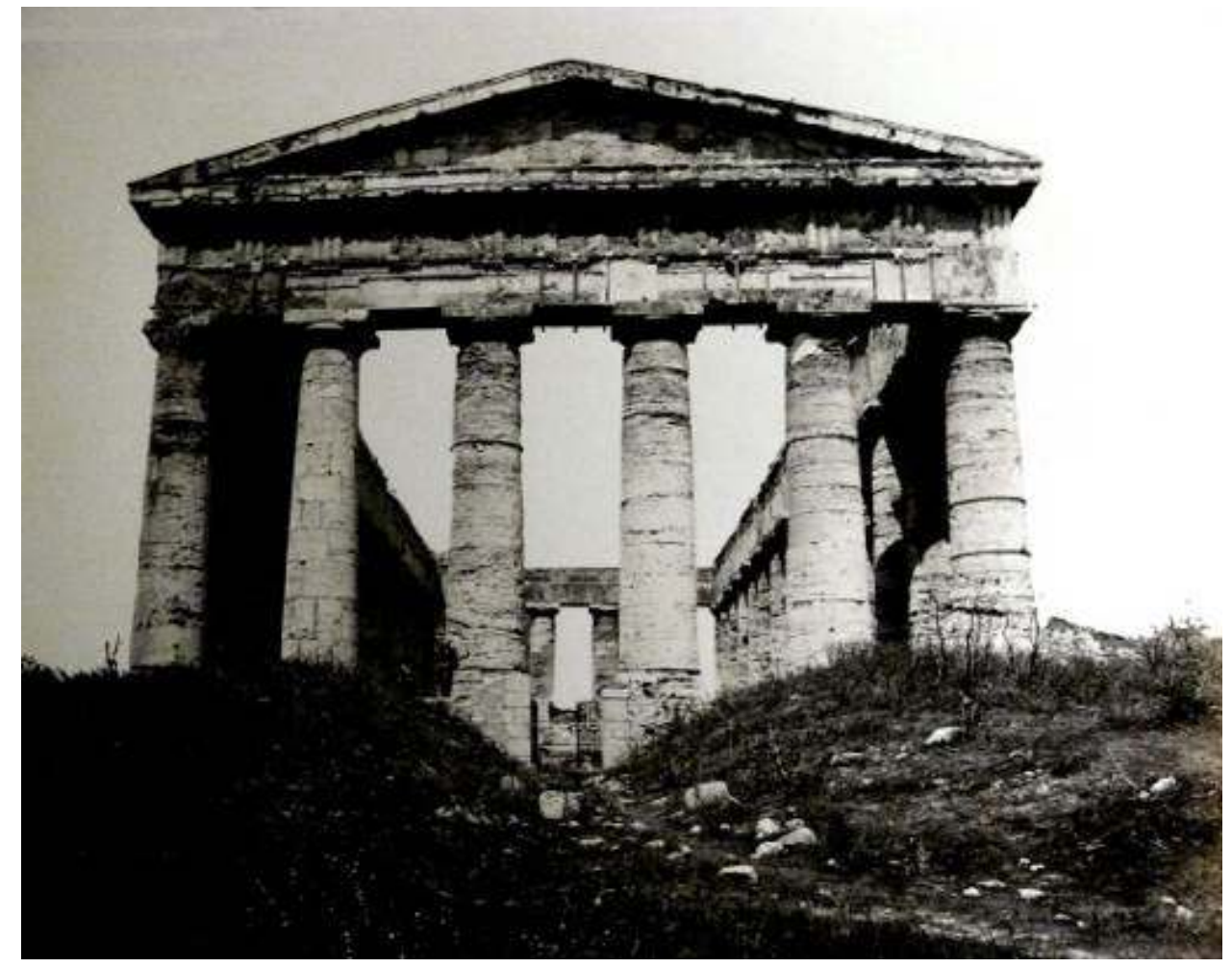

Fig. 3 : Giorgio Sommer [attr.], Tempio di Segesta, vers 1870, tirage sur papier albuminé, Paris, Bibliothèque nationale de France, département des Estampes et de la photographie, collection $\mathrm{A}$. Armand, AD-34 (A, 7)-FOL. 
Mais dans d'autres cas on ne peut alléguer cet alibi "contextualisant " et cette répartition fonctionnelle entre les diverses techniques de représentation, tant le monument se fond dans la matière même de l'image : dans telle vue générale d'Avignon par Baldus ou de Berne par Bisson, il n'y a pour ainsi dire plus d'architecture; les monuments se décentrent et se dissolvent dans le paysage urbain, deviennent de simples éléments de la composition - jusqu'au paysage pur, sans plus aucun édifice (jardin romain par Caneva, oasis algérienne par Beaucorps). L'intention artistique, affirmée par le photographe et sans nul doute validée par le collectionneur, est encore plus nette dans les admirables séries de vues de Constantinople par Robertson et Beato [fig. 4], ou d'Espagne par Clifford [fig. 5], qui constituent des fleurons de la collection. Leur nombre même prouve qu'Armand les a délibérément retenues pour leur qualité esthétique propre. C'est bien l'image photographique en tant que telle qui est ici recherchée et valorisée.

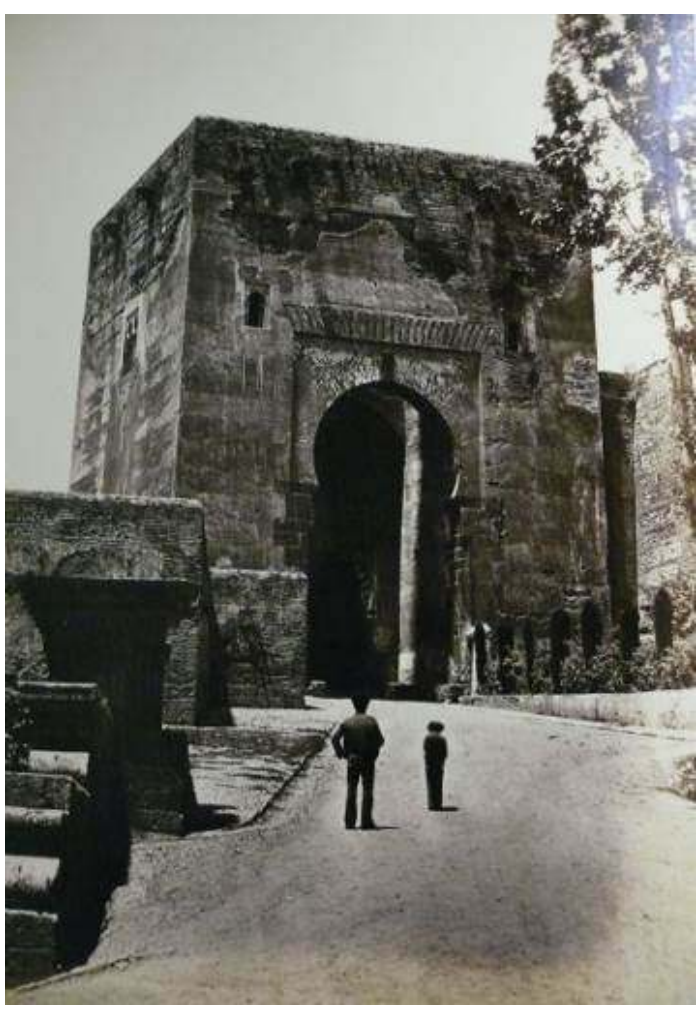

Fig. 4 : Charles Clifford, Porte du Jugement, entrée de l'Alhambra de Grenade, vers 1862, tirage sur papier albuminé, Paris, Bibliothèque nationale de France, département des Estampes et de la photographie, collection A. Armand, AD-34 (A, 215)-FOL. 


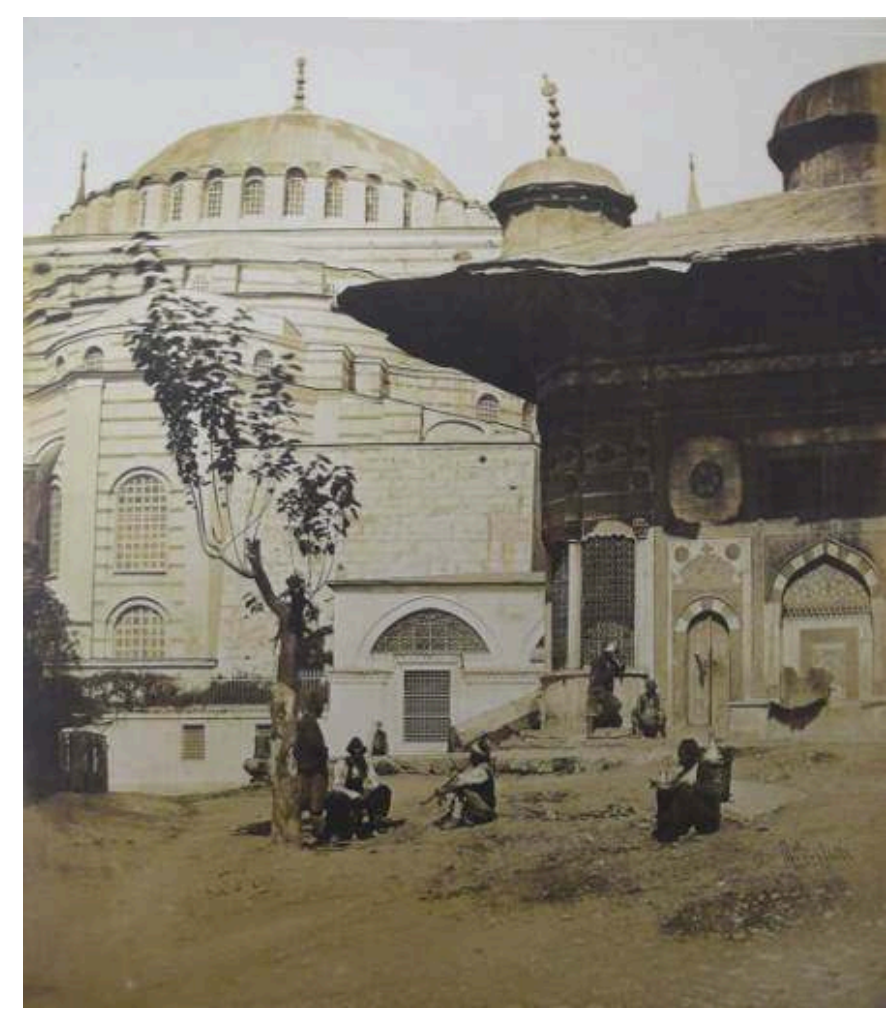

Fig. 5 : James Robertson, Fontaine d'Ahmet III et abside de Sainte-Sophie à Istanbul, vers 1855, tirage sur papier albuminé, Paris, Bibliothèque nationale de France, départements des Estampes et de la photographie, collection A. Armand, AD-34 (A, 160)-FOL.

En effet, comme nous l'avons vu, la collection témoigne qu'Armand s'est intéressé précocement à la photographie, dès le début des années 1850 , pendant une décennie où celle-ci semblait gagner sa reconnaissance en tant qu'art. Il a dû fréquenter les salons, les expositions, peut-être les photographes eux-mêmes, et surtout acquis des photographies qui ne représentaient pas des œuvres d'art ou des monuments, mais avaient été saluées comme des réussites exceptionnelles de l'art photographique naissant : en premier lieu les marines et les vues du camp de Châlons par Gustave Le Gray $^{7}$. Autrement dit, il ne s'est pas seulement intéressé précocement à la valeur documentaire de la photographie, mais a aussi été très tôt sensibilisé à son esthétique propre.

En ce sens, s'il serait anachronique de voir en Armand un des premiers collectionneurs de photographies au sens où nous l'entendons aujourd'hui, sa collection constitue une des toutes premières histoires photographiques de l'art. Certes il existait des entreprises éditoriales antérieures : la mission héliographique bien sûr, les albums de Blanquart-Évrard, les séries architecturales de Piot ou des frères Bisson, pour ne parler que de la France. Mais avec Armand, on se place du côté de la réception, par quelqu'un qui n'est pas photographe mais architecte, qui plus est architecte de la génération d'avant la photographie, formé à d'autres types de représentations. Par l'ampleur et la qualité globale de l'iconographie rassemblée, la collection Armand marque donc la reconnaissance et l'avènement de la photographie comme médium, mais aussi, inconsciemment ou subrepticement, comme art, ou en tout cas comme proposition visuelle spécifique et originale, donnant à voir, de l'architecture et au-delà, quelque chose qui lui appartient en propre. 


\section{BIBLIOGRAPHIE}

Sylvie AUBENAS et Paul-Louis ROUBERT (dir.), Primitifs de la photographie. Le calotype en France, 1843-1860, Paris, Gallimard / Bibliothèque nationale de France, 2010, p. 267.

Laure BEAUMONT-MAILLET, « Les collectionneurs au Cabinet des estampes », Nouvelles de l'estampe, nº 132, décembre 1993.

Emmanuelle BRUGEROLLES (dir.), De Michel-Ange à Géricault : dessins de la donation Armand-Valton, [cat. expo. Paris, École nationale supérieure des beaux-arts, 19 mai-12 juillet 1981], Paris, École nationale supérieure des beaux-arts, 1981 .

Emmanuelle BRUGEROLLES, Les Dessins de la collection Armand-Valton : la donation d'un grand collectionneur du XIX siècle à l'École des Beaux-arts, Paris, École nationale supérieure des beaux-arts, 1984.

François COURBOIN, Inventaire des dessins, photographies et gravures relatifs à l'histoire générale de l'art, légués au Département des estampes de la Bibliothèque nationale par M. A. Armand, Lille, L. Danel, 1895. Georges DUPLESSIS, Notice sur M. Alfred Armand, architecte, Paris, Plon, Nourrit et Cie, 1888.

Tiphaine ZIRMI, Alfred Armand (1805-1888), un architecte collectionneur, thèse d'archiviste paléographe sous la direction de Sylvie Aubenas et Jean-Michel Leniaud, École nationale des Chartes, 2003.

\section{NOTES}

1. Voir Laure BEAUMONT-MAILlET, «Les collectionneurs au Cabinet des estampes ", Nouvelles de l'estampe, $\mathrm{n}^{\circ}$ 132, décembre 1993.

2. Sur Alfred Armand, la source principale est la nécrologie écrite par Georges DUPLESSIS (Notice sur M. Alfred Armand, architecte, Paris, Plon, Nourrit et Cie, 1888). Sa biographie, son œuvre d'architecte et sa carrière de collectionneur ont fait l'objet en 2003 d'une remarquable thèse d'archiviste paléographe par Tiphaine ZIRMI, (Alfred Armand (1805-1888), un architecte collectionneur, sous la direction de Sylvie Aubenas et Jean-Michel Leniaud, École nationale des Chartes).

3. Voir Emmanuelle BRUGEROLLES (dir.), De Michel-Ange à Géricault : dessins de la donation ArmandValton, [cat. expo. Paris, École nationale supérieure des beaux-arts, 19 mai-12 juillet 1981], Paris, École nationale supérieure des beaux-arts, 1981 et Emmanuelle BRUGEROLLES, Les Dessins de la collection Armand-Valton: la donation d'un grand collectionneur du XIX siècle à l'École des Beaux-arts, Paris, École nationale supérieure des beaux-arts, 1984.

4. L'ensemble de la collection est conservé sous la cote AD-34 (A)-FOL.

5. Inventaire des dessins, photographies et gravures relatifs à l'histoire générale de l'art, légués au Département des estampes de la Bibliothèque nationale par M. A. Armand, Lille, L. Danel, 1895.

6. Voir Sylvie AUBENAS et Paul-Louis ROUBERT (dir.), Primitifs de la photographie. Le calotype en France, 1843-1860, Paris, Gallimard / Bibliothèque nationale de France, 2010, p. 267.

7. La collection contenait en tout 47 photographies de Le Gray, dont quelques vues de Paris et reproductions de tableaux ; elles ont depuis été transférées dans l'œuvre de Gustave Le Gray (cote RESERVE EO-13) dans le cadre d'une reconstitution et d'un rassemblement de cette œuvre jusque-là dispersée dans les collections. 


\section{AUTEUR}

\section{THOMAS CAZENTRE}

Bibliothèque nationale de France, département des Estampes et de la photographie 


\title{
L'album du Santa Lucía
}

\author{
Photographier la perspective urbaine
}

\section{Amari Peliowski}

1 Jusqu'au milieu du XIxe siècle, plus de $80 \%$ de la population du Chili habitait en milieu rural. Santiago, sa capitale, était encore à cette époque une agglomération villageoise, vivant dans l'héritage de trois siècles de colonialisme. La formation de la République à partir de 1810, l'essor de l'industrie minière et l'arrivée de colonies étrangères dans la deuxième moitié du siècle ont stimulé la formation d'une oligarchie qui, pour sa part, a poussé le pays à la libéralisation économique et politique; ce phénomène a eu pour conséquence un fort développement de l'émigration de la campagne vers les villes, et notamment vers Santiago. En 1875, la ville couvrait 1500 hectares et comptait 120000 habitants (en 1778, on recensait 25000 habitants). Santiago dut ainsi entamer un processus de transformation afin de loger une population croissante: le réseau d'éclairage au gaz existant depuis 1848 fut remplacé par l'électricité à partir de 1883; quelques rues furent pavées; la gare ferroviaire principale fut rénovée en 1897 selon un projet d'Eiffel pour la compagnie française Schneider du Creusot, tandis que deux autres grandes gares furent construites seulement au début $\mathrm{du} \mathrm{xx}^{\mathrm{e}}$ siècle. Contrastant avec les constructions basses en adobe (pisé) de l'époque coloniale, la deuxième moitié $\mathrm{du}$ siècle vit la construction de palais aristocratiques dans le centre-ville, inaugurant une ségrégation sociale par quartier, qui s'est maintenue jusqu'à aujourd'hui. Bien qu'une nouvelle classe moyenne se soit formée et que le pays ait connu un essor économique - grâce surtout à l'exploitation du salpêtre à partir des années 1870 -, des quartiers périphériques misérables se sont formés à l'extrême nord et à l'extrême sud de la capitale, avec pour conséquences des problèmes d'hygiène et de santé, devenus un vrai fléau social vers la fin du siècle ${ }^{1}$.

2 Benjamín Vicuña Mackenna (1831-1886)², homme politique, historien et écrivain chilien, devenu en 1872 gouverneur de Santiago - poste qu'il occupa jusqu'en 1875 -, est aujourd'hui reconnu pour ses projets de modernisation urbaine de la capitale ; il est même considéré comme le premier urbaniste chilien pour son adhésion à des principes d'urbanisme européen et par l'inscription de ses projets dans un discours progressiste, phénomènes l'un et l'autre inédits à cette époque au Chili. Grâce à la prospérité économique du pays, il put proposer aux autorités gouvernementales un vaste plan de 
renouvellement de Santiago, incluant la construction de nouveaux parcs, l'installation de sculptures et de fontaines dans la ville, la construction d'un boulevard de ceinture, la canalisation du fleuve Mapocho, la création d'un grand parc urbain, la construction de nouvelles cités pour les plus démunis, l'installation de locaux de détente destinés à restreindre l'activité des chinganas - tavernes rustiques situées dans la périphérie -, la construction de halles, d'écoles et d'un nouvel abattoir. Son projet phare - et une des seules œuvres qu'il a réussi à concrétiser - est le réaménagement du mont Santa Lucía en promenade et parc de loisirs. Ses projets urbanistiques étaient notamment motivés par son admiration pour Paris, ville qu'il avait visitée pendant son exil entre 1852 et 1856, en échappant à une condamnation à mort suite à son opposition au gouvernement du président Manuel Montt. Dans la capitale française, il avait particulièrement apprécié les travaux du baron Haussmann, les principes hygiénistes appliqués par ce dernier et par le préfet Rambuteau, ainsi que les aménagements paysagers de Jean-Charles Alphand, notamment le parc des Buttes-Chaumont ${ }^{3}$.

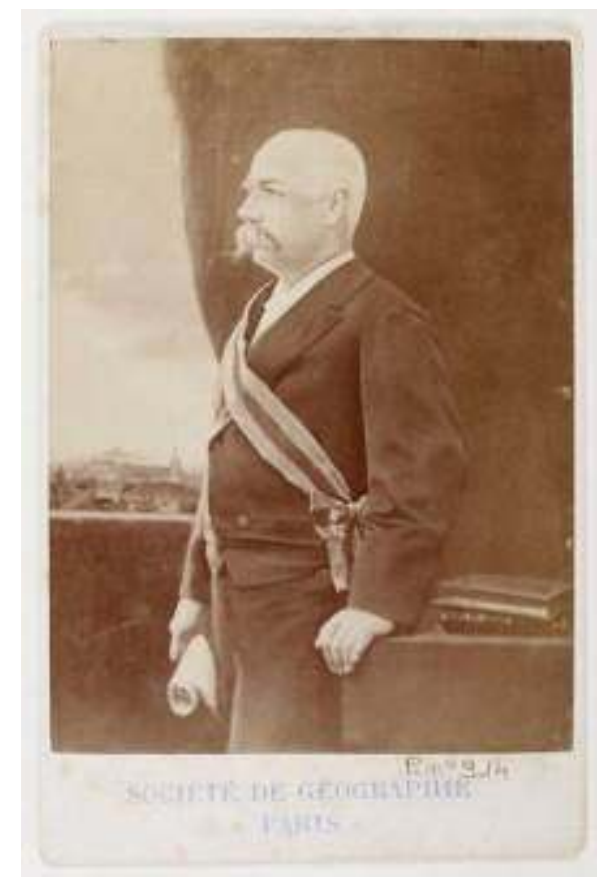

Fig. 1 : Leblanc y Adaro, Benjamín Vicuña Mackenna, 1883, Paris, Bibliothèque nationale de France.

3 En accord avec le mouvement francophile qui s'est développé au Chili et dans la majorité des pays latino-américains pendant la deuxième moitié $\mathrm{du}$ xix ${ }^{\mathrm{e}}$ siècle et les premières décennies $\mathrm{du} \mathrm{xx}^{\mathrm{e}}-\mathrm{la}$ Révolution française constituant un modèle idéologique pour les apôtres de l'indépendance de l'empire espagnol-, Vicuña Mackenna songeait à faire de Santiago un petit Paris latino-américain.

4 En 1874, Vicuña Mackenna a inauguré la promenade du mont Santa Lucía, un parc aménagé sur les versants du mont le plus important de la ville. En 1541, Pedro de Valdivia avait fondé Santiago aux pieds de ce mont, à côté du fleuve Mapocho. Le mont, qui revêtait une dimension sacrée pour les habitants originaires du lieu - les Mapuche, qui appelaient le mont Huelén-, était resté pendant la période coloniale une proéminence rocheuse inhabitée. À part quelques fortifications, l'installation de petites carrières pour le pavage de la ville et l'établissement en 1856 d'un observatoire astronomique, le mont n'avait pas été utilisé comme espace de loisirs pour les santiaguinos et n'avait pas non plus été absorbé par la croissance urbaine. Ainsi, à part 
les fortifications et les carrières éparses, le mont a abrité un cimetière de bandits et a été utilisé comme dépotoir : des fonctions qui montrent le caractère marginal de cet espace dans la ville. La promenade de 1874 , inspirée du paysagisme romantique français, fut conçue par Vicuña Mackenna comme l'exploitation d'un espace dont Santiago n'avait pas encore profité ; pour lui, cette refondation était destinée à devenir le symbole d'une ville moderne.

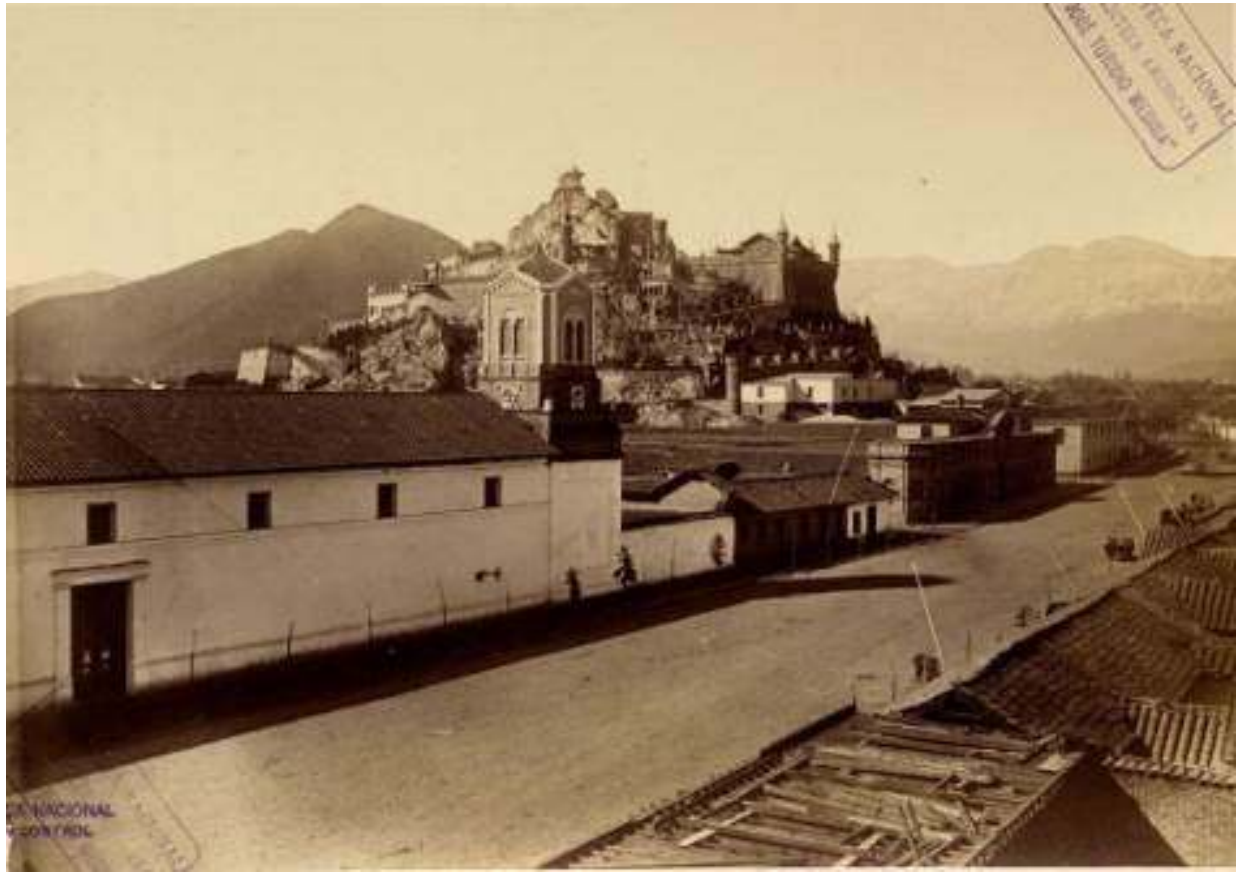

Fig. 2 : P.-E. Garreaud, Deuxième vue générale [Segunda Vista General], planche II, Álbum del Santa Lucía, 1874, Santiago, Archivo Visual de Santiago.

5 Le réaménagement du mont comportait la création de plusieurs voies pour piétons et carrosses, d'escaliers, de terrasses et de belvédères, d'un ermitage, et aussi d'un restaurant, d'un musée d'art précolombien et d'une bibliothèque, le tout décoré de sculptures en pierre et en bronze. La construction de la promenade, une expression du triomphe du culturel sur le naturel ${ }^{4}$, était bien un symptôme des temps : on peut dire que l'architecture devenait, au cours du xix siècle, le véhicule essentiel du désir de modernisation de la nouvelle République. La rénovation architecturale de Santiago s'accompagna de la création du titre d'architecte du gouvernement - chargé de diriger les chantiers d'architecture publique - pendant les années 1840. L'architecture fut aussi stimulée par la fondation, en 1849, du premier cours dédié aux futurs architectes, dirigé par le français Claude Brunet-Debaines à l'université du Chili. Pour Vicuña Mackenna, « l'anthropisation» des espaces naturels, l'inclusion d'œuvres d'art et l'aménagement d'espaces de détente constituaient une manière d'assainir la ville, en opposition à la force invasive, rustique et barbare des chimbas (quartiers misérables périphériques), de s'éloigner des marques impériales encore visibles dans la ville et d'imposer les idéaux de progrès qui provenaient de France et d'Angleterre. Cependant, les idées les plus marquantes pour Vicuña Mackenna avaient probablement été celles de son contemporain l'argentin Domingo Faustino Sarmiento, qui prônait l'idéal de la civilisation - issu de l'imaginaire colonialiste $d u$ xvIII $^{e}$ siècle - par opposition à la barbarie "exécrable» des indigènes ${ }^{5}$. Le projet du Santa Lucía était pour Vicuña Mackenna une manière d'établir un modèle urbain pour la nation en développement. 
Selon ses propres mots, la promenade devait être appréciée comme une expression du " sens moderne du mot promenade, qui signifie loisir et art, santé et hygiène ${ }^{6}$ ".

Pour faire de la propagande et surtout pour se défendre des critiques (il était appelé «le fou du Santa Lucía»), Vicuña Mackenna publia en 1874 l'Álbum del Santa Lucía, composé de ses propres écrits ${ }^{7}$ et d'une série de quarante-huit photographies prises par Pierre Émile Garreaud (1835-1875) ${ }^{8}$. Ce photographe français, né à Metz, est arrivé au Pérou en 1855 avec son compatriote le photographe Amic Gazan. Installés à Lima, ils établirent ensemble un atelier de photographie qui fonctionna jusqu'en 1858. Garreaud, qui continua à travailler seul après le départ d'Amic en 1858 (appelant dorénavant son atelier E. Garreaud et Cia.), devint rapidement le principal promoteur du collodion humide, en remplacement du daguerréotype. Vers 1865, encouragé probablement par son succès au Pérou, il déménagea au Chili, où il ouvrit des ateliers de photographie dans plusieurs villes : Copiapó, La Serena, Santiago, Valparaíso, Talca et Concepción.

7 À son arrivée, des photographes étaient déjà installés au Chili depuis quelques années : le premier atelier de daguerréotypie avait été inauguré à Valparaíso en 1843. Garreaud fut sans doute l'un des plus importants photographes et promoteurs de la photographie pendant les dernières décennies $\mathrm{du} \mathrm{XIX}^{\mathrm{e}}$ siècle au Chili, travaillant sur de multiples formats et techniques dans ses boutiques et publiant des photographies dans les principaux journaux de Santiago et de Valparaíso. En 1869, il publia son premier album chilien de photographies de paysages, intitulé Vues de la Patagonie, du Détroit [de Magallanes] et de la Terre du Feu ${ }^{10}$.

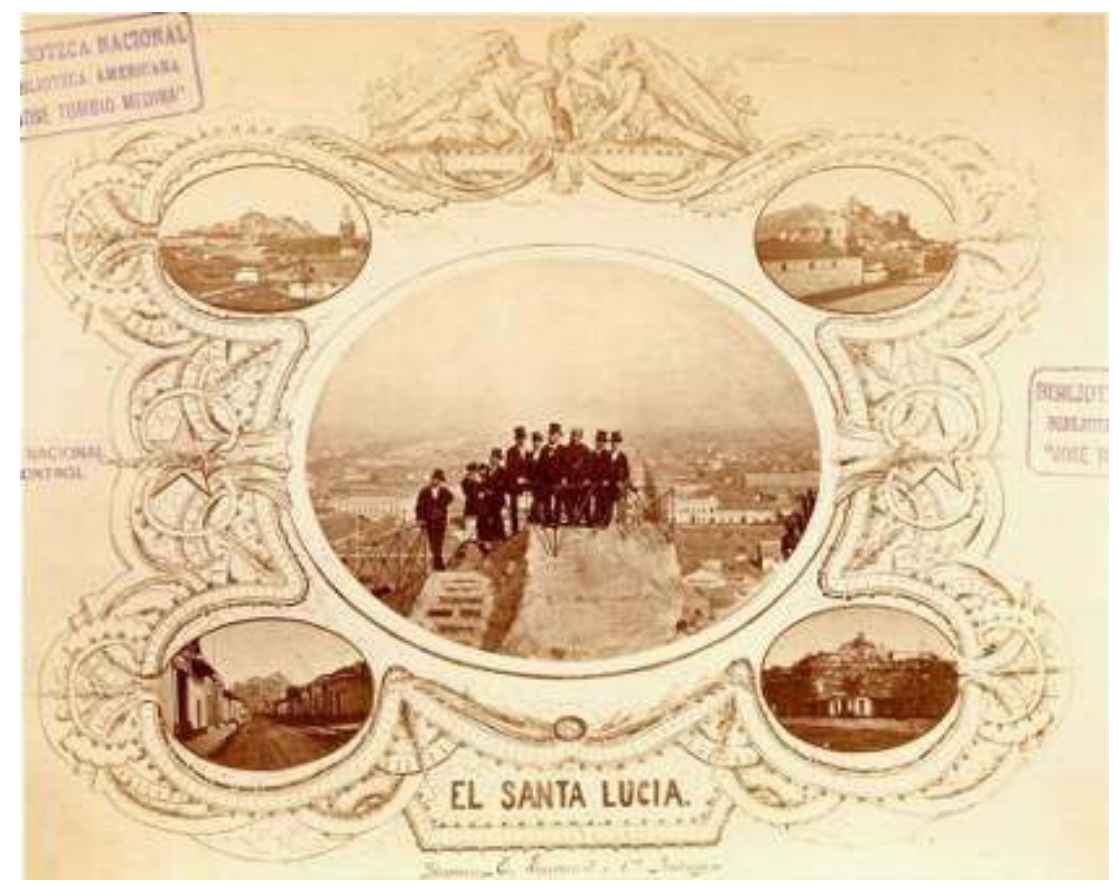

Fig. 3 : P.-E. Garreaud, couverture de l'Álbum del Santa Lucía, 1874, Santiago, Archivo Visual de Santiago.

La publication de l'album du Santa Lucía fut une première au Chili pour plusieurs raisons : bien qu'il s'inscrive dans une tradition - celle de promouvoir les bienfaits du pays par des œuvres figurées ${ }^{11}-$, l'album du Santa Lucía était le premier à faire la promotion par l'image d'une œuvre d'urbanisme. Il fut suivi par des albums représentant notamment Santiago et Valparaíso : Album : Vues de Valparaíso (188?), du Français Félix Leblanc; Chili à jour: Album graphique de vues du Chili (1915-20), de Hume 
\& Walker; Album de Santiago et vues du Chili (1915), de Jorge Walton; et Valparaíso Panoramique (1924), de Roberto Hernández. Autre caractéristique inédite : l'album du Santa Lucía était destiné aux habitants de Santiago et aux autorités d'autres villes chiliennes, afin qu'elles s'en inspirent pour des projets semblables, et non aux étrangers d'Europe ou d'Amérique du Nord où se publiaient au XIX et au début du $\mathrm{xx}^{\mathrm{e}}$ siècle des recueils écrits, dessinés et photographiés destinés à encourager le tourisme et l'investissement au Chili.

Parmi les quarante-huit vues de l'album, on trouve une douzaine d'images formant un panorama circulaire de la ville autour du mont; dans les écrits de Vicuña Mackenna, il est question, sans doute de façon erronée, d'un diorama (au lieu de panorama) : «Cette sixième perspective complète la précédente et sert à développer l'étonnante formation circulaire du Santa Lucia dans toute sa circonférence accidentée [...] C'est pour cela que l'artiste a procédé correctement, en contournant le mont avec l'objectif de son appareil, dans le but de le montrer dans cet album comme un véritable diorama sur une toile sans fin $^{12}$ ». Cette attention pour le panorama se traduit aussi dans le soin avec lequel les terrasses du mont ont été placées, offrant une vue de Santiago à 360 degrés ${ }^{13}$. Vicuña Mackenna et Garreaud ont suivi, avec l'inclusion de ces vues panoramiques, la tendance à escalader le mont et faire des portraits urbains, tendance qui a commencé en 1821 quand le capitaine anglais William Waldegrave a dessiné un panorama de la ville depuis le Santa Lucía, poursuivi après par quelques photographes, graveurs et peintres étrangers et locaux ${ }^{14}$.

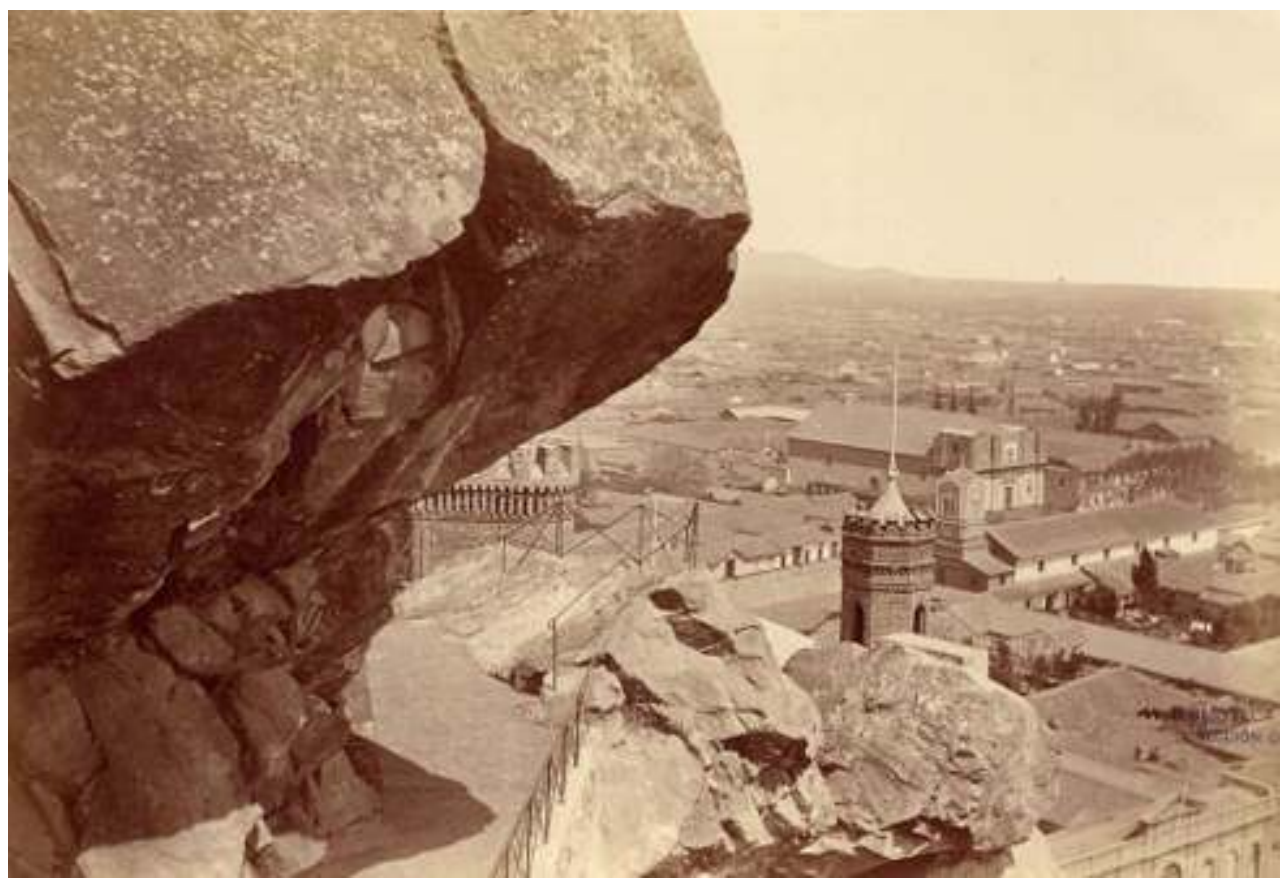

Fig. 4 : P.-E. Garreaud, Vue de la plaine du Maipo [Vista del Ilano de Maipo], planche VIII, Álbum del Santa Lucía, 1874, Santiago, Archivo Visual de Santiago. 


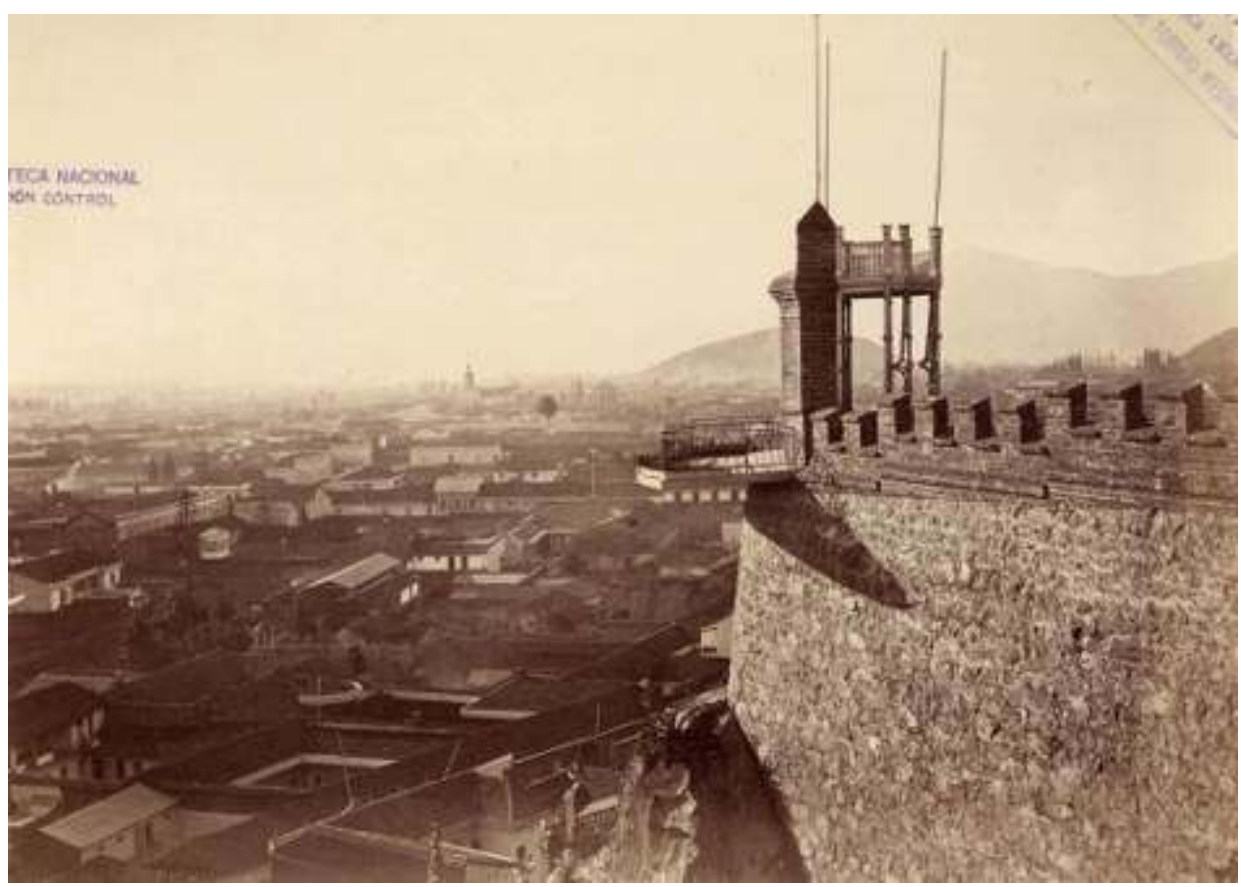

Fig. 5 : P.-E. Garreaud, Le balcon volant [El balcon volado], planche XXVI, Álbum del Santa Lucía, 1874, Santiago, Archivo Visual de Santiago.

Avec l'inauguration de cette promenade, les citoyens communs (plutôt les aristocrates) et non plus seulement les cartographes, les artistes, les carriers ou les sujets marginaux qui fréquentaient le mont auparavant, accédèrent pour la première fois au belvédère de leur ville, à une vue totale seulement perceptible jusqu'alors sur les cartes. Parcourir le mont et ses différents sites se transformait ainsi en une double démarche qui se voulait moderne : l'acte presque médical de respirer l'air frais et de se promener parmi des sculptures, des musées et des restaurants ; mais aussi un acte de possession visuelle et même, pourrait-on dire, de contrôle panoptique de la ville. En fait, la notion de surveillance visuelle de la ville s'inspirait de ce que Vicuña Mackenna avait observé dans les villes européennes. La distance depuis le belvédère permettait d'observer l'organisation de la ville, telle qu'il l'avait vu dans le Paris de Haussmann et telle qu'il souhaitait le renforcer à Santiago avec la construction d'un boulevard de ceinture qui, selon les idéaux ségrégationnistes de l'Argentin Sarmiento, séparait la ville civilisée (le centre) de la ville barbare (la périphérie).

D'autre part, comme le démontrent les premiers panoramas de Robert Barker en 1787 et les représentations urbaines de Schinkel au début du XIX siècle, la ville s'est transformée, au tournant du siècle, en paysage et en spectacle. Comme Walter Benjamin l'a noté, la ville du xix $x^{e}$ siècle, et notamment Paris, s'est caractérisée par l'exaltation du désir visuel d'objets et de marchandises. Ainsi, des phénomènes comme les flâneries ou la mise en scène des vitrines des passages parisiens et, plus subtilement, l'invention de la photographie et du cinéma, et la construction des perspectives urbaines exploitées dans les tracés du baron Haussmann, peuvent se comprendre à travers l'idée de séparation moderne entre l'observateur - aussi spectateur ou acheteur - et l'objet regardé15.

12 La vue d'en haut a été aussi symptomatique de la rapide croissance démographique des villes et des progrès techniques. En premier lieu, le regard distancié depuis le belvédère renvoie au désir de séparation et de distinction d'une foule croissante et pressante, 
celle qu'Edgar Allan Poe a bien décrite en $1840^{16}$. En deuxième lieu, regarder la ville d'en haut ou depuis le lointain était un acte qui est devenu possible et attirant grâce à l'aérostation, l'aviation et la construction en hauteur: du premier daguerréotype de Daguerre sur un toit du boulevard du Temple jusqu'aux tableaux vertigineux de Paris par Caillebotte, des photographies de perspectives tordues de Rodtchenko jusqu'aux photographies pseudo-aériennes de Moholy-Nagy, la vue aérienne semble bien avoir influencé l'art de la seconde moitié du XIX ${ }^{e}$ siècle et les premières décennies du $\mathrm{Xxe}^{17}$. Nous pouvons ainsi inscrire l'album du Santa Lucía dans cette trajectoire d'ascension verticale - ou de cartographisation du regard - des représentations picturales et photographiques des villes ${ }^{18}$.

Si la photographie au XIX ${ }^{\mathrm{e}}$ siècle a été avant tout considérée pour sa valeur documentaire, comme l'établit la fameuse maxime de Barthes, "ça a été ${ }^{19}$ ", ou si pendant les dernières cinquante années la photographie a été plutôt considérée comme un moyen d'expression subjectif de la réalité (qu'on peut caractériser avec la phrase «ça a été, mais ce n'était pas ça " proposée par Jean-Claude Lemagny) ${ }^{20}$, dans le cas des photographies de l'album du Santa Lucía on peut parler aussi d'un enregistrement du désir de ce qui arrivera, un "ça sera » symbolisé par la vue d'en haut, vers le lointain : une nouvelle perspective de la ville moderne, depuis la ville.

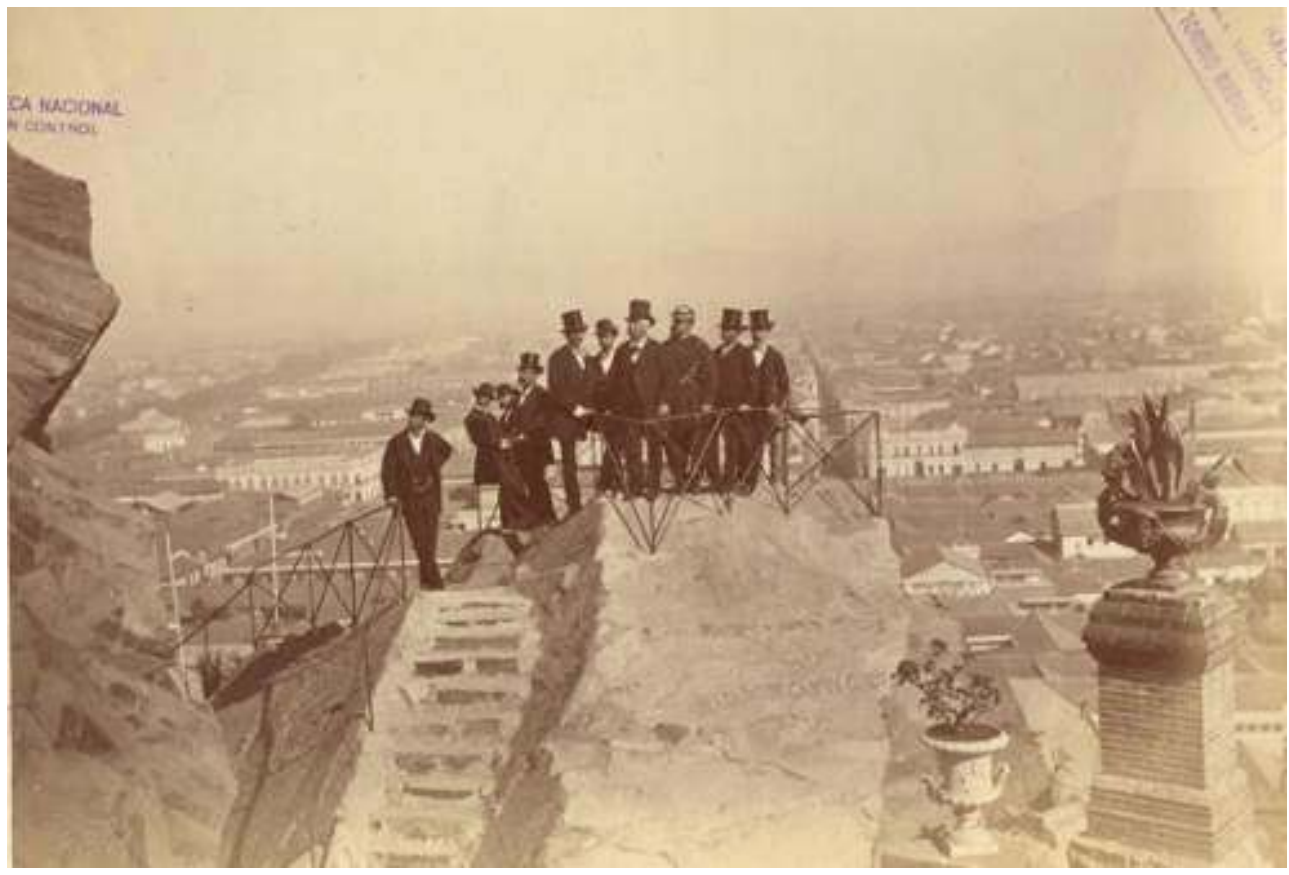

Fig. 6 : P.-E. Garreaud, La roche Tarpéienne [La roca Tarpeya], planche XXVIII, Álbum del Santa Lucía, 1874, Santiago, Archivo Visual de Santiago.

C'est dans ce sens qu'on peut analyser le cliché La roche Tarpéienne, qui apparaît aussi sur la couverture. On peut y voir une tentative par la photographie de fixer les idéaux que Vicuña Mackenna cherchait à diffuser. On y voit l'un des endroits du mont aménagé où passé et futur s'imbriquent : un rocher " architecturé » par une balustrade et un escalier - dont le nom fait probablement référence à l'ancienne fonction du mont $^{21}$ - et une place baptisée, comme on le voit sur le côté du rocher, "place des Champs-Élysées ", emblème de la ville récemment modernisée par Haussmann. On voit sur la photographie un panorama de Santiago, située au loin, et dix personnages juchés sur ce rocher, dont les quatre premiers de droite à gauche (le quatrième est Benjamín 
Vicuña Mackenna) expriment une attitude solennelle en regardant vers le mont, comme s'ils contemplaient un futur prometteur symbolisé par les œuvres du Santa Lucía. Les six autres personnages ont une attitude plus ambiguë, presque détendue : quelques-uns regardent directement vers l'appareil photographique, un peu distraits ; les autres s'appuient confortablement sur la balustrade et regardent le mont ou la ville lointaine. Ils semblent, on pourrait le dire, moins convaincus par cette promesse de modernité, censée améliorer les conditions de vie précaires dans la ville.

\section{BIBLIOGRAPHIE}

Roland BARTHES, La chambre claire. Note sur la photographie, Paris, Gallimard, 1980.

Walter BENJAMIN, Illuminations, New York, Schoken Books, 1969.

Walter Benjamin, Le livre des passages (1935), Paris, Éditions du Cerf, 1997.

Walter BENJAMIN, L'œuvre d'art à l'époque de sa reproductibilité mécanisée (1936), Paris, Éditions Allia, 2003.

Soledad CHAVEZ, « De resemantizaciones, remedos y reminiscencias. El cerro Santa Lucía y su intervención », dans Artelogie, n 3, été 2012.

Guy DEBORD, La société du spectacle, Paris, Buchet/Chastel, 1967.

Armando De RAMON, Santiago de Chile. Historia de una sociedad urbana (Santiago, Editorial Sudamericana, 2000), Santiago, Catalonia, 2015.

Germán HIDALGO, « Panoramic view and national identity. Two of Santiago de Chile's public spaces in the second half of the nineteenth century ", Planning perspectives, vol. 24, 3 juillet 2009, p. 319-347.

Angela LAMPE (dir.), Vues d'en haut, cat. exp., Metz, Centre Pompidou-Metz, 2013, Metz, Centre Pompidou-Metz Éditions, 2013.

Jean-Claude LEMAGNY, L'ombre et le temps. Essais sur la photographie comme art, Paris, Nathan, 1992.

Amari PELIOWSKI et Catalina VALDÉs, « Escalas de Visión. Lecturas para la colección virtual de imágenes archivovisual », Mouseion, $\mathrm{n}^{\circ}$ 13, 2012, p. 51-74.

Edgar Allan PoE, « L'homme des foules », dans Nouvelles histoires extraordinaires (1840), Paris, M. Lévy frères, 1875.

Hernán RODRIGUEZ, Historia de la fotografía. Fotógrafos en Chile durante el siglo XIX, Santiago, Centro Nacional del Patrimonio Fotográfico, 2001.

Benjamín VICUÑA MACKENNA, La transformación de Santiago: notas e indicaciones respestuosamente sometidas a la Ilustre Municipalidad, al Supremo Gobierno y al Congreso Nacional. Por el Intendente de Santiago, Santiago, Imprenta de la Librería del Mercurio, 1872. 
Benjamín VICUÑA MACKENNA, Álbum del Santa Lucía. Colección de las principales vistas, monumentos, jardines, estatuas $i$ obras de este paseo, dedicado a la Municipalidad de Santiago por su actual presidente, B. Vicuña Mackenna, Santiago, Imprenta de la Librería del Mercurio, 1874.

Leslie WeHNER, Benjamín Vicuña Mackenna: génesis de la transformación de Santiago, thèse de doctorat, Santiago, université pontificale catholique du Chili, 2000.

\section{NOTES}

1. Pour une description des problèmes de ségrégation et d'hygiène à Santiago dans la deuxième moitié du XIX ${ }^{\mathrm{e}}$ siècle, voir Armando De Ramón, Santiago de Chile. Historia de una sociedad urbana (Santiago, Editorial Sudamericana, 2000), Santiago, Catalonia, 2015, p. 188-193.

2. Pour une biographie détaillée de Vicuña Mackenna, voir Leslie Wehner, Benjamín Vicuña Mackenna: génesis de la transformación de Santiago, thèse de doctorat, Santiago, université pontificale catholique du Chili, 2000.

3. Pour plus d'informations sur les plans de Vicuña Mackenna pour Santiago, voir Benjamín Vicuña Mackenna, La transformación de Santiago: notas e indicaciones respestuosamente sometidas a la Ilustre Municipalidad, al Supremo Gobierno y al Congreso Nacional. Por el Intendente de Santiago, Santiago, Imprenta de la Librería del Mercurio, 1872.

4. Pour une analyse détaillée de l'aménagement du mont Santa Lucía comme instrumentalisation de la nature au service de l'urbanisme, voir Soledad Chàvez, « De resemantizaciones, remedos y reminiscencias. El cerro Santa Lucía y su intervención », dans Artelogie, nº 3, 2012 [consulté le 28 janvier 2016].

5. Sarmiento a exposé ces idées notamment dans son roman Facundo, écrit au cours de son deuxième exil au Chili (titre original : Civilización i barbarie: Vida de Juan Facundo Quiroga. Aspecto físico, costumbres i hábitos de la República Argentina, Santiago, Imprenta del Progreso, 1845).

6. « [El cerro Santa Lucía] constituye un verdadero paseo, en el sentido moderno de la palabra, que significa recreo i arte, salud e hijiene [sic]». Benjamín Vicuña Mackenna, Álbum del Santa Lucía. Colección de las principales vistas, monumentos, jardines, estatuas i obras de este paseo, dedicado a la Municipalidad de Santiago por su actual presidente, B. Vicuña Mackenna, Santiago, Imprenta de la Librería del Mercurio, 1874, p. 6.

7. Les écrits décrivent en détail, entre autres aspects, les attractions de la promenade, les dépenses et l'ingénierie de l'œuvre, et les noms des collaborateurs.

8. L'album est consultable en ligne [consulté le 28 janvier 2016].

9. Pour plus d'informations sur l'activité photographique au Chili au XIX siècle, consulter Hernán Rodriguez, Historia de la fotografía. Fotógrafos en Chile durante el siglo XIX, Santiago, Centro Nacional del Patrimonio Fotográfico, 2001.

10. Vistas de la Patagonia, del Estrecho y de la Tierra del Fuego, Valparaíso, Imprenta del Mercurio de Valparaíso, 1869. Un autre album de photographies «territoriales » est Andenken an Chile, du Français Eugène Maunoury, réalisé entre 1860 et 1870.

11. Les exemples les plus importants d'albums sur le Chili qui ont précédé celui du Santa Lucía furent l'Atlas de l'histoire physique et politique du Chili (Atlas de la historia física y política de Chile, Paris, E. Thunot, 1854), dans lequel son auteur Claude Gay a inclus des centaines de gravures de paysages, de personnages typiques, de plantes, d'animaux et de minéraux du territoire chilien; l'album Vues de la Patagonie, du Détroit [de Magallanes] et de la Terre du Feu, du photographe Pierre Émile Garreaud (voir n. 8) ; et le livre Chile ilustrado : guía descriptiva del territorio de Chile, de las capitales de Provincia, de los puertos principales (Valparaíso, Librerías y ajencias del Mercurio, 1872), Paris, Impr. Hispano-Americana de Rouge Dunan i Fresne, 1872. 
12. «Esta sesta perspectiva completa la precedente i sirve para desarrollar la admirable formación circular del Santa Lucía en toda su accidentada circunferencia [...] Por esto el artista ha procedido acertadamente al circumbalar el cerro con el foco de su máquina, a fin de exhibirlo en este Álbum como un verdadero diorama en una tela sin fin [sic] ». Mackenna 1874, pl. VI.

13. Un article très intéressant sur la vue panoramique comme modèle de visualisation pour penser l'espace public à Santiago pendant le xix ${ }^{\mathrm{e}}$ siècle est celui de l'architecte Germán Hidalgo, «Panoramic view and national identity. Two of Santiago de Chile's public spaces in the second half of the nineteenth century ", Planning perspectives, vol. 24, $\mathrm{n}^{\circ} 3$, juillet 2009, p. 319-347.

14. Nous parlons du petit nombre (pas plus de quinze) de peintures, gravures et photographies représentant Santiago depuis le haut du Santa Lucía et réalisées avant 1874 ; par exemple, Claudio Gay et Johann Moritz Rugendas (1854), James Melville Gillis (1855), T. R. Harvey (c. 1860), Giovatto Molinelli (1861), le Français Pedro (Pierre) Dejean (1867) et les photographies du Français Eugène Manoury prises entre 1860 et 1870, entre autres.

15. Benjamin développe ces idées notamment dans Le livre des passages (1935), Paris, Éditions du Cerf, 1997 et dans L'œuvre d'art à l'époque de sa reproductibilité mécanisée (1936), Paris, éditions Allia, 2003. Guy Debord, pour sa part, exploite l'idée de spectacularisation de la société moderne dans La société du spectacle, Paris, Buchet/Chastel, 1967.

16. Edgar Allan Poe, "L'homme des foules », dans Nouvelles histoires extraordinaires (1840), Paris, M. Lévy frères, 1875.

17. L'exposition « Vues d'en haut » a abordé la thématique de l'influence de la vue aérienne dans l'art moderne et contemporain. Angela Lampe (dir.), Vues d'en haut, cat. exp., Metz, Centre Pompidou-Metz, 2013, Metz, Centre Pompidou-Metz Éditions, 2013.

18. Pour une analyse plus approfondie de ce phénomène de cartographisation du regard dans la représentation de Santiago pendant le $\mathrm{xIX}^{\mathrm{e}}$ siècle, voir Amari Peliowski et Catalina Valdés, «Escalas de Visión. Lecturas para la colección virtual de imágenes archivovisual.cl », Mouseion, $\mathrm{n}^{\circ}$ 13, 2012, p. 51-74.

19. Roland Barthes, La chambre claire. Note sur la photographie, Paris, Gallimard, 1980.

20. À partir des années 1960 , plusieurs artistes ont utilisé la photographie pour remettre en question l'efficacité mimétique de la photographie par rapport à la représentation de la réalité. Par rapport à la représentation de l'espace architectonique et urbain, quelques artistes comme Jan Dibbets, Gordon Matta-Clark, Michael Heizer et Robert Smithson ont utilisé pendant les années 60 et 70 la photographie pour montrer l'artifice et la subjectivité de l'espace photographique perspectif. Voir Gilles Tiberghien, Land Art, Paris, Éditions Carré, 1993.

21. Bien que l'analogie est évidente, Vicuña Mackenna ne fait pas mention du réfèrent de la roche Tarpéienne, lieu d'exécution de criminels à Rome jusqu'à la fin de la République romaine. Voir Benjamín Vicuña Mackenna, Álbum del Santa Lucía. Colección de las principales vistas, monumentos, jardines, estatuas i obras de este paseo, dedicado a la Municipalidad de Santiago por su actual presidente, B. Vicuña Mackenna, Santiago, Imprenta de la Librería del Mercurio, 1874, p. 14.

\section{AUTEUR}

\section{AMARI PELIOWSKI}

École des hautes études en sciences sociales 


\section{La restauration du Gros-Horloge de Rouen (1889-1893) par Louis Charles Sauvageot}

Un chantier à l'épreuve de la photographie

Sophie Nasi

« Le Gros-Horloge est l'un des monuments rouennais les plus curieux et à de multiples points de vue », écrivait Jules Adeline en 1894, résumant ainsi la difficulté de définir un ensemble stylistiquement hétérogène et pourtant constitué d'éléments étroitement liés historiquement et matériellement ${ }^{1}$. Situés au cœur du vieux Rouen, un arc et un pavillon Renaissance construits entre le $\mathrm{xIV}^{\mathrm{e}}$ et le $\mathrm{xVIII}^{\mathrm{e}}$ siècles, constituent le GrosHorloge proprement dit, auxquelles se sont ajoutées une loggia à étages et une fontaine d'angle, le tout groupé autour du beffroi municipal proche de l'ancien hôtel de ville. 


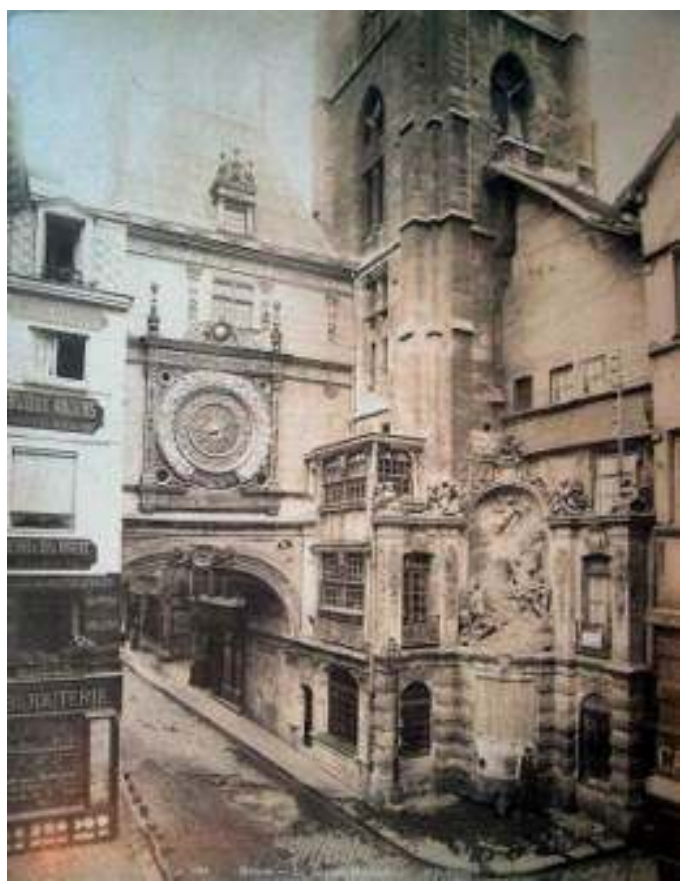

Fig. 1 : Vue du Gros-Horloge après restauration (face ouest), photographie, Charenton-le-Pont, Médiathèque de l'architecture et du patrimoine, album Durand

2 Dans les années 1820, la réputation du Gros-Horloge dépassait les frontières de la France. Tandis que la ville était livrée aux « démolisseurs » désireux de faire disparaittre les rues tortueuses et les vieux logis en pans de bois, les voyageurs et les artistes se pressaient autour de cet ensemble dissymétrique, patiné par le temps et si pittoresque. Un dessin de Bonington exécuté en 1824 et immédiatement reproduit et diffusé par la gravure d'Engelmann en reste le témoignage le plus précoce et le plus célèbre. Sous la Monarchie de Juillet, la Renaissance est remise au goût du jour ; aussi les architectes et les archéologues ne manquèrent-ils pas de s'intéresser à l'arc sculpté de Rouen ${ }^{2}$. En 1862 le pavillon et sa boutique étaient classés sur la liste des monuments historiques. Puis, en 1889, vint le tour de la fontaine datée du XVIII ${ }^{e}$ siècle.

Dans l'esprit des Rouennais, le monument occupait une place toute particulière : il était d'autant plus précieux à leurs yeux qu'il était étroitement lié à l'histoire politique de la ville et à ses libertés municipales. Lorsque la mairie entreprit sa restauration en 1889 , les enjeux étaient de taille et divers car, si les élus locaux voyaient dans cette opération un acte politique destiné à valoriser l'emblème des libertés communales, il s'agissait pour la commission des Monuments historiques de rendre son éclat à l'un des monuments majeurs de la Renaissance. De ce point de vue, le choix du maître d'œuvre est révélateur puisqu'à cette date, Louis Sauvageot (1842-1908) ${ }^{3}$ était considéré comme l'un des spécialistes de la Renaissance en Normandie ${ }^{4}$ et comme l'un des meilleurs praticiens parmi ceux attachés au service des Monuments historiques. De fait, celui-ci s'efforça de mener à bien ce chantier suivant une rigueur toute scientifique.

\section{Au cœur de l'histoire communale}

4 Le site du Gros-Horloge s'organise autour du beffroi situé à l'angle de la rue des Vergetiers et de la rue du Gros-Horloge, face à l'ancien hôtel de ville abandonné à la Révolution ${ }^{5}$. Ce bâtiment inachevé, projeté au xvII siècle par Jacques II Gabriel, 
remplaçait déjà un édifice municipal plus ancien. Il était relié à la tour du beffroi par un corps de passage construit au-dessus de la rue pendant la première moitié du $\mathrm{xVI}^{\mathrm{e}}$ siècle, constitué d'une arche richement ornée et surmontée d'un pavillon en pans de bois. C'est là que prenaient place les cadrans de l'horloge. Au pied du beffroi, une fontaine était venue occuper l'angle rentrant formé par la boutique et la tour.

5 Le beffroi avait été au Moyen Âge l'emblème de la puissance de la Commune dans les villes où les bourgeois se gouvernaient eux-mêmes. Rouen se flattait d'avoir obtenu en premier ce privilège et les historiens locaux prétendaient que, dès le $\mathrm{xII}^{\mathrm{e}}$ siècle, un premier édifice s'élevait, signe matériel de son affranchissement. Ses cloches rivalisaient avec celles des églises : la Cache-Ribaud sonnait le couvre-feu tandis que la Rouvel appelait les Rouennais aux assemblées et signalait les dangers. À la suite de la révolte de 1382 dite de la Harelle, le roi Charles VI abolit la Commune et fit démolir ce beffroi primitif. Vers 1390, l'architecte Jehan Ier de Bayeux édifia l'actuelle tour à l'emplacement même de la précédente pour célébrer l'indépendance retrouvée de la ville. C'est là que furent installés les mécanismes de l'horloge. On adossa les premiers cadrans aux murailles de la Porte Massacre. Moins d'un siècle plus tard, celle-ci fut démolie et elle fit place à l'actuelle arche en anse de panier construite entre 1525 et 1527. Sur l'intrados de la voûte, un décor devenu célèbre fut sculpté, figurant l'agneau des armes de Rouen dans une représentation du Bon Pasteur ${ }^{6}$.

6 La disparition de la porte Massacre avait mis à nu la façade nord du beffroi située entre la nouvelle arche et la fontaine du Massacre établie en 1457 pour distribuer les eaux de la source Gaalor. Pour combler cet espace vide, on construisit une étroite tourelle dont l'escalier donnait accès au premier étage de l'arche où avaient été placés les mécanismes de l'horloge. Une petite boutique lui fut accolée à laquelle l'ajout de deux étages supplémentaires en 1623 valut le nom de loggia. Enfin, l'architecte Defrance édifia en 1732 la fontaine dite d'Alphée et d'Aréthuse (selon Les Métamorphoses d'Ovide) pour remplacer celle du $\mathrm{Xv}^{\mathrm{e}}$ siècle ${ }^{7}$.

7 Telle est, brièvement évoquée, l'histoire complexe des éléments qui forment le GrosHorloge, une histoire hautement symbolique aux yeux des Rouennais et que n'ont pas manqué de souligner les historiens locaux successifs ${ }^{8}$. Ce qui unit cet ensemble de constructions hétérogènes, c'est la commande par la municipalité rouennaise. Dans une ville où le siège de l'administration a plusieurs fois été déplacé, le site illustre la pérennité de l'histoire urbaine et constitue une expression forte et rassurante de la stabilité des institutions communales. Bien que les éléments aient tous été construits après la perte de l'autonomie municipale de 1382, ils sont situés en lieu et place d'édifices plus anciens dont ils apparaissent comme les héritiers directs. Ils constituent donc un témoignage du glorieux passé rouennais. On comprend alors pourquoi tandis que les archéologues et les architectes des années 1880-1900 s'intéressaient plus spécifiquement au pavillon Renaissance et à son arche, les érudits locaux se consacraient d'une part à l'étude du beffroi et de ses cloches et d'autre part, à celle de l'horloge et des cadrans'.

\section{Les enjeux d'une restauration}

8 La disposition générale du Gros-Horloge à la fin du XIX ${ }^{e}$ siècle correspondait à peu près à celle qui est reproduite par Aubin-Louis Millin dans l'article XXX des Antiquités Nationales $^{10}$. Mais le monument avait perdu une grande partie de son lustre et dès 1864 , 
deux ans après le classement, Eustache de La Quérière faisait appel à la sollicitude de la commune $^{11}$. Il fallut pourtant attendre la fin des années 1870 pour que les élus manifestent l'intention de restaurer le Gros-Horloge. Mais tout au plus s'agissait-il alors de rendre sa fonction technique à l'édifice par la remise en marche du système d'horloge des cadrans. Le conseiller municipal Ernest Fauquet attira sans résultat l'attention de ses collègues du conseil municipal sur l'état de l'édifice en mai 1877, puis à nouveau en 1888. Suite au vœu émis en novembre 1886 par la commission départementale des Antiquités de voir les cadrans retrouver leur usage, l'horloger municipal, Robert Louis Hainaut, fut chargé de rédiger un rapport sur la question ${ }^{12}$. À partir des observations formulées par celui-ci, l'architecte de la ville Jules Touzet dressa un projet de restauration des dits cadrans en mars $1887^{13}$. La Société des Amis des monuments rouennais, consultée pour donner son avis sur cette question, suggéra la première d'élargir le projet à la restauration de l'ensemble du monument ${ }^{14}$. Les études furent alors poursuivies en ce sens, d'autant qu'en octobre 1888, Touzet signalait l'état de dégradation inquiétant de la voûte et des façades du pavillon qu'on avait pu constater lors de l'estampage des sculptures destinées aux collections du musée du Trocadéro.

9 Le conseil municipal prit alors les choses en main et au début de l'année 1889 une commission consultative chargée d'étudier plus précisément la question fut nommée ${ }^{15}$. Le rapport en date du 2 février 1889 concluait à la nécessité d'effectuer immédiatement les travaux indispensables à la conservation du monument et proposait également de rendre au Gros-Horloge son caractère primitif, en reconstituant certaines parties disparues offrant un intérêt archéologique et artistique. Afin d'accélérer le processus, Louis Ricard intervint personnellement auprès de la direction des Beaux-arts, demandant un secours en vue "d'assurer la conservation d'un des plus anciens et des plus remarquables monuments de la Ville de Rouen: le monument de nos libertés communales, Le Gros-Horloge $»^{16}$. Usant de son influence auprès des divers acteurs du ministère tout au long de l'année 1889, cet ancien maire de Rouen, entré à la Chambre des députés aux côtés de la gauche républicaine progressiste en 1886, obtint rapidement l'assurance d'une subvention ministérielle ${ }^{17}$.

Le 25 février 1889, l'architecte Louis Sauvageot était désigné comme maître d'œuvre du chantier, un choix propre à satisfaire les partis en présence. Aux yeux de la commission des Monuments historiques sa nomination était une garantie de réussite dans une entreprise de restauration qui s'avérait délicate. En outre, en un temps où le recrutement d'architectes locaux avait la faveur de l'opinion publique, le choix de Sauvageot fit l'unanimité au sein du conseil municipal. En effet, l'homme avait dirigé, entre 1871 et 1882, le bureau d'architecture de Rouen. Son nom restait attaché à la politique de grands travaux qui avait largement contribué à modifier l'aspect de la ville conformément aux nécessités de la vie moderne ${ }^{18}$. Il évoquait également un certain nombre de constructions nouvelles comme l'église Saint-Hilaire (1874-1878), le muséebibliothèque (1876-1891) et le théâtre des Arts (1876-1882). Nommé Architecte du gouvernement en 1882, Sauvageot consacra dès lors l'essentiel de sa carrière à la restauration, gravissant un à un les échelons du service des Monuments historiques et du service des Édifices diocésains ${ }^{19}$. Parfaitement inséré dans les réseaux locaux, il mit à profit les liens tissés durant ses années rouennaises. Ainsi, c'est sur la proposition de son prédécesseur Desmarest que la direction des Cultes lui avait confié le diocèse de Rouen en 1881. Fort de son expérience, il était en 1889 une personnalité respectée dans 
le domaine : d'où sa présence au sein de la commission consultative nommée par le conseil municipal pour étudier l'état du Gros-Horloge.

\section{Une restauration symbolique et consensuelle}

11 Louis Sauvageot remit son rapport à la commission des Monuments historiques dès le 15 novembre 1889. Celui-ci fut adopté dans son ensemble le 7 février 1890. Fin avril, la dépense évaluée à plus de 58000 francs était répartie entre les intervenants ${ }^{20}$. Les négociations se déroulèrent dans un climat harmonieux et le projet ne donna lieu à aucun débat particulier. De même, le choix des entreprises qui allaient collaborer à la restauration du Gros-Horloge, rassurait le ministère attaché à la compétence des entrepreneurs et le conseil municipal soucieux de fournir du travail aux artisans locaux. En août 1890, les adjudications des principaux lots eurent lieu ${ }^{21}$. Le Rouennais Henri Gosselin, proche collaborateur de Sauvageot, fut nommé inspecteur des travaux $^{22}$.

12 Suivant la vision convenue $\mathrm{du} \mathrm{XIX}^{\mathrm{e}}$ siècle, l'intérêt des édifices Renaissance résidait avant tout dans le traitement des détails d'ornementation tandis que l'on voyait souvent des défauts dans la composition générale des bâtiments. Sauvageot n'échappait pas à la règle :

«Par l'originalité et la liberté de la composition, le beau caractère accentué et la richesse exubérante de la sculpture, le Gros Horloge constitue incontestablement un splendide exemple de l'architecture décorative de l'époque de la Renaissance. Il montre, en outre, avec ses deux énormes cadrans, le plus important spécimen, croyons-nous, de plomberie d'art du Xvi ${ }^{e}$ siècle conservé dans notre pays ${ }^{23}$. "

Mais l'architecte respecta la dissymétrie de l'édifice et opta pour une reprise générale du bâtiment qui enjambe la rue, de la boutique et de sa loggia ${ }^{24}$. Il avait prévu également les réparations nécessaires à la tour du beffroi contenant l'horloge et la sonnerie, bien que celle-ci ne fût pas classée, ce dont personne ne s'étonna. En revanche, la fontaine $\mathrm{du} \mathrm{xvIII}^{\mathrm{e}}$ siècle dont Sauvageot reconnaissait certes le mérite artistique, fut laissée en état ${ }^{25}$. Le gardien du beffroi et sa famille logeaient dans le pavillon même de l'horloge qui avait subi diverses modifications dénaturant l'intérieur de l'édifice. Plusieurs fenêtres informes percées dans la façade furent bouchées et un plancher intermédiaire installé à mi-hauteur du cadran fut supprimé.

En dehors de ces travaux confortatifs, l'architecte proposa également toute une série de restitutions. Elles étaient censées, selon l'inspecteur général des Monuments historiques Selmersheim, remettre l'édifice dans son « état primitif ${ }^{26}$ ». Pour le conseil municipal, elles devaient lui rendre "sa première splendeur ${ }^{27}$ ». Autant de nuances de vocabulaire qui rappellent les formules ambiguës utilisées par Viollet-le-Duc dans son projet pour Notre-Dame de Paris. Les découvertes archéologiques allaient apporter leur caution à ces travaux que l'urgence ne pouvait justifier. Sauvageot obtint de la commission l'autorisation d'établir des échafaudages sur le monument afin d'étudier la structure, dont on ignorait tout, ainsi que les restes de la décoration peinte et sculptée du bâtiment de l'horloge. Il put constater que le pavillon au-dessus de l'arche en pierre était en fait composé de pans de bois dont une partie était recouverte par la plomberie des cadrans et le surplus par du plâtre décoré de pilastres, de frises et de corniches, moulés et appliqués sur cet enduit. Il releva également de nombreuses traces de peinture et de dorure sur les diverses parties de l'édifice. 
15 Le caractère exceptionnel de l'intervention était dès lors justifié. Sauvageot allait s'employer à faire du Gros-Horloge un spécimen de l'architecture de la Renaissance selon la conception du XIX ${ }^{\mathrm{e}}$ siècle. L'organisation générale de la décoration en plâtre du pavillon était encore perceptible et s'organisait autour de deux étages de pilastres et de contre-pilastres, ornés de balustres et d'arabesques et reliés par des entablements ornés de frises. Les détails subsistaient en assez grand nombre et une étude méticuleuse des édifices civils rouennais datant de la Renaissance, qui offrait le même type d'ordonnance, allait permettre à l'architecte de recréer ce décor ${ }^{28}$. Les contre-pilastres en pierre ornés de balustres de l'hôtel de Romé, contemporain du Gros-Horloge, étaient pratiquement similaires à ceux retrouvés à l'étage supérieur du pavillon ${ }^{29}$. Ils servirent de modèle pour compléter les parties disparues et ce, malgré la différence de matériau $^{30}$. Les parties en plomb de l'horloge furent également restaurées et le maître d'œuvre reprit la disposition d'ensemble grâce aux vestiges de la façade est en meilleur état de conservation que celle de l'ouest. Dès lors, il ne restait plus qu'à rendre au pavillon ses couleurs primitives. Les restes étaient suffisamment nombreux pour éviter toute interprétation dans la restauration : «Les cadrans étaient d'azur avec chiffres dorés, les nuages d'un blanc grisâtre semé d'étoiles d'or; les fonds des frises et des pilastres d'un ton vert, sauf les disques et moutons, se détachaient sur du vermillon; les moulures de l'entablement, les ornements et feuillages étaient dorés ${ }^{31}$.» Enfin, Sauvageot fit appliquer une patine pour atténuer l'éclat et la brillance des nouvelles couleurs et simuler déjà un certain vieillissement ${ }^{32}$.

16 La restauration devait également permettre d'apporter quelques améliorations au mécanisme de l'horloge, selon le vœu de la municipalité rouennaise. Le devis prévoyait le rétablissement $\mathrm{du}$ dispositif $\mathrm{du}$ cadran astronomique comprenant les phases lunaires, l'indication de l'heure et ce que l'on appelait alors les roues du zodiaque. Après s'être assuré que l'ensemble était encore en état de marche, cette opération aurait dû se réduire à une affaire d'horloger. Or, une fois encore, des découvertes archéologiques vinrent modifier l'état des connaissances et justifier de nouveaux travaux. En effet, le petit orifice ménagé dans la partie basse de chaque cadran s'ouvrait sur un disque supportant des bas-reliefs dont on pensait à tort qu'ils représentaient les mois de l'année. Le mouvement était bloqué. Mais lorsque les roues furent dégagées, l'architecte comprit que les bas-reliefs se référaient en fait aux jours de la semaine, figurés sous la forme de représentations des dieux ${ }^{33}$. Malgré les altérations subies par les deux roues, Sauvageot parvint à reconstituer cet ensemble en plomb doré et ciselé.

17 Les campagnes de restauration des édifices du Moyen Âge et de la Renaissance dans la seconde moitié du $\mathrm{XIX}^{\mathrm{e}}$ siècle faisaient "une large part au décor des toits ${ }^{34}$ »; elles contribuèrent à la diffusion d'un goût pour la décoration métallique dans la construction contemporaine. La mise en œuvre d'une nouvelle toiture sur le GrosHorloge s'inscrit dans ce contexte. Certains travaux effectués en 1819 avaient modifié l'aspect du monument. La crête et les épis de faîtage notamment avaient été déposés puis remplacés par deux boules. Sauvageot décida de revenir à l'état précédent à partir des modèles fournis par la gravure de Millin et la description faite par Eustache de La Quérière qui affirmait avoir vu la toiture avant sa destruction. L'ardoise du comble du pavillon fut remplacée par une couverture en plomb et les décors des deux petites lucarnes furent rétablis. Pour autant, les sources précédemment évoquées ne constituèrent pas une contrainte pour l'architecte qui imagina un couronnement de style Renaissance, mais remis au goût du jour. Selon Eustache de La Quérière, «le 
comble de l'arcade du Gros-Horloge était décoré d'une crête accompagnée de deux épis au sommet desquels on voyait la représentation du soleil et de la lune. Au centre de la crête, il existait un troisième épi portant les armes de la ville ${ }^{35}$ ». L'œuvre de Sauvageot reprenait ce principe de composition mais offrait un aspect plus grandiose que l'original: les deux épis latéraux mesuraient $3,50 \mathrm{~m}$ de hauteur. Elle matérialisait également le souvenir des décors en plomb dont Rouen était riche au début du siècle et qui avaient pratiquement tous disparu. L'exécution de cet ensemble fut confiée aux ateliers Monduit, alors considérés dans ce domaine comme le «leader incontestée ${ }^{36}$ ».

\section{L'album Durand}

Dès le premier devis remis en novembre 1889, une somme de près de 950 francs était prévue par l'architecte pour financer la campagne photographique qui devait accompagner la restauration des bas-reliefs en plomb doré et ciselé des cadrans astronomiques ${ }^{37}$. Au vu de l'état de dégradation de ces décors, Sauvageot souhaitait faire photographier les différentes scènes et confronter les vestiges encore en place sur chacun des cadrans, afin de recréer les parties manquantes dans leurs formes et couleurs $^{38}$. Il précise d'ailleurs dans son devis qu'il a besoin de vues «à une assez grande échelle pour pouvoir y noter toutes les indications actuelles de la décoration peinte et dorées $(\mathrm{sic})^{39} \%$. Or, la campagne photographique prit une ampleur bien différente avec l'avancement du chantier, ce que confirme le décompte des travaux déposé à la fin de l'année 1892. En effet, c'est finalement un ensemble de clichés beaucoup plus vaste qui fut réalisé, non seulement des scènes des deux roues du zodiaque, mais plus globalement, des vues illustrant l'état avant et après la restauration et permettant de suivre le chantier dans ses différentes phases.

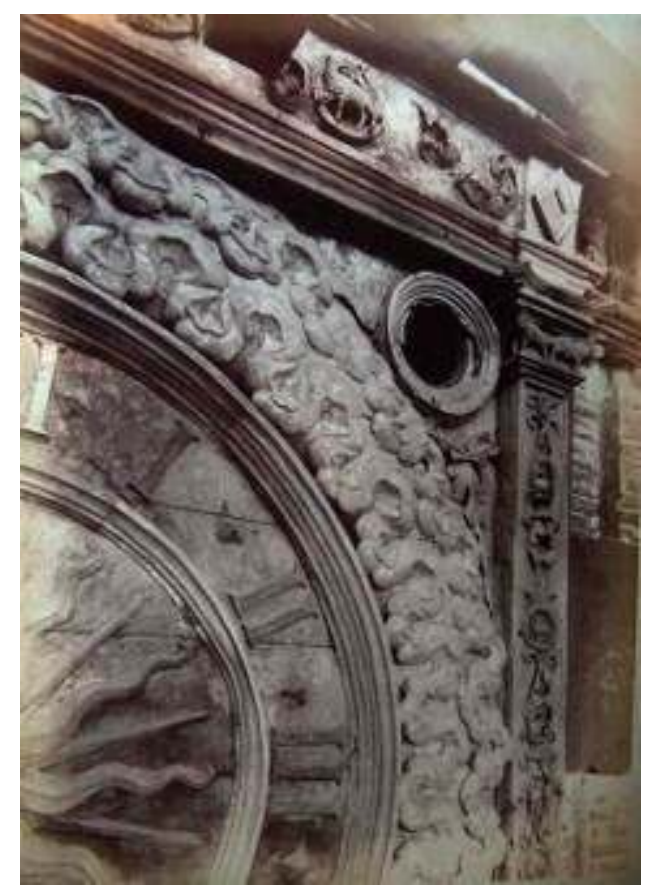

Fig. 2 : Le cadran de l'horloge avant restauration (détail), photographie, Charenton-le-Pont, Médiathèque de l'architecture et du patrimoine, album Durand. 


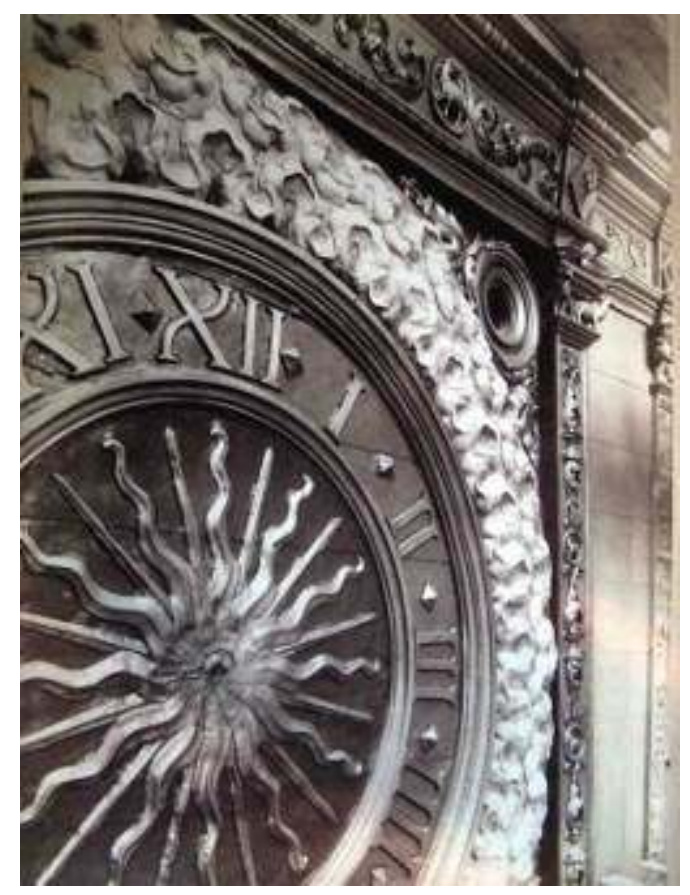

Fig. 3 : Le cadran de l'horloge après restauration (détail), photographie, Charenton-le-Pont, Médiathèque de l'architecture et du patrimoine, album Durand.

Les circonstances ayant amené Sauvageot à modifier son dessein premier, simplement utilitaire, et à utiliser la photographie comme outil documentaire, témoin de son travail, restent floues.

L'album photographique (appelé «Album Durand» du nom de son auteur), déposé au service des Monuments historiques, se compose de 28 tirages mesurant $30 \mathrm{~cm}$ sur $40 \mathrm{~cm}$, ayant servi aux études générales de la restauration du monument ${ }^{40}$. Il est complété par un second jeu d'épreuves montrant les scènes des roues du zodiaque et utilisé pour la notation des traces des anciennes colorations. L'ensemble est l'œuvre de Jean Eugène Durand (1845-1929), sous-chef de bureau aux Monuments historiques et photographe attaché au même service, qui couvrit l'ensemble du territoire entre 1876 et 1917 pour enrichir le fonds photographique du ministère. Il existe également un second album conservé par les descendants de l'architecte, qui regroupe les mêmes vues à taille plus réduite, ainsi que quelques épreuves montrant Sauvageot ou encore le maître-ferronnier Ferdinand Marrou, prenant la pose devant les nouveaux décors en plomb du cadran. Autant d'images plus intimes qui témoignent de l'humeur d'un chantier et lui donnent vie.

21 À défaut de pouvoir préciser le calendrier des prises de vue, il est certain que le photographe s'est rendu à plusieurs reprises à Rouen, deux voire peut-être trois fois : avant le début des travaux pour prendre des photographies d'ensemble et de détails des cadrans (couronnement, moulures en plomb et en plâtre, bas-reliefs en plomb), puis au moment de la pose des nouveaux décors. 


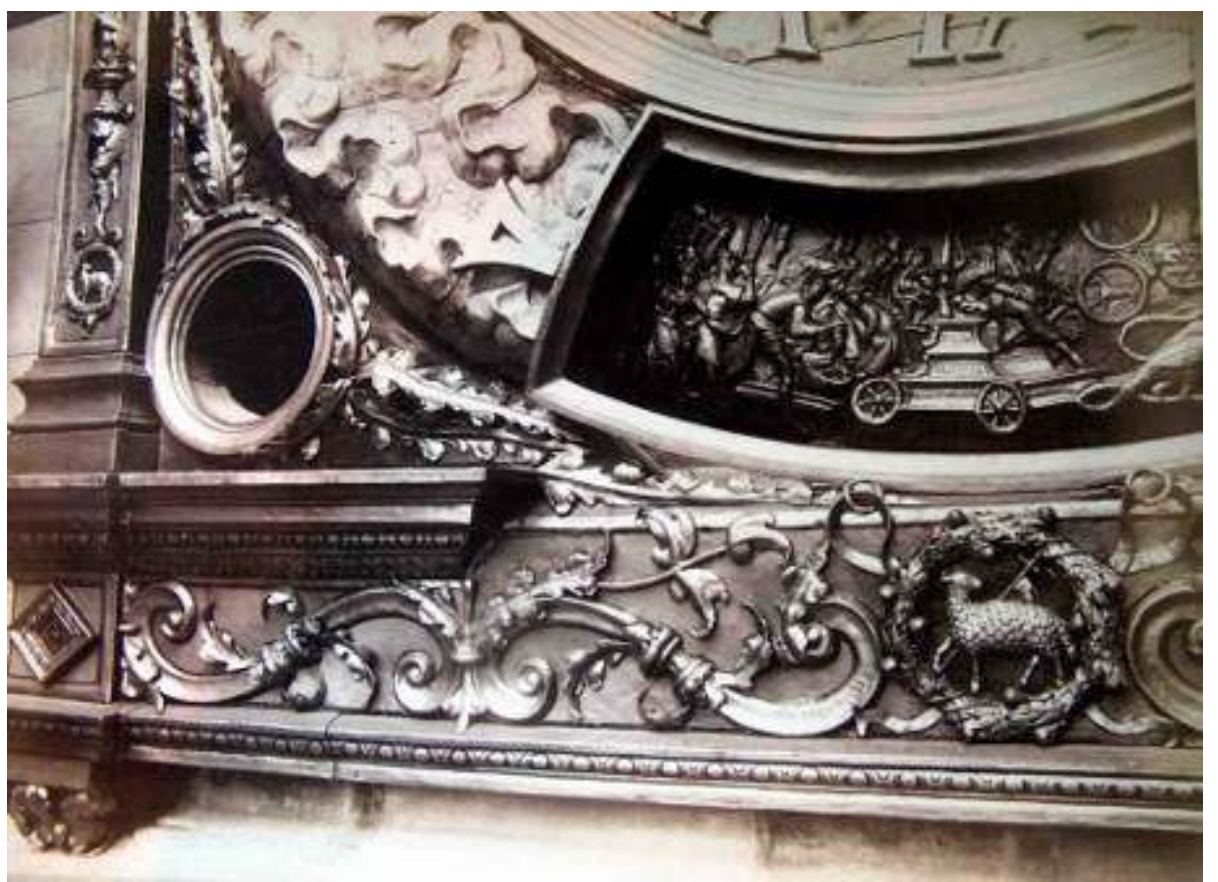

Fig. 4 : Le cadran de l'horloge après restauration (détail de la partie basse), photographie, Charenton-lePont, Médiathèque de l'architecture et du patrimoine, album Durand.

22 Le chantier ayant débuté en mai 1890 par le gros œuvre, il se poursuivit avec la restauration des anciens plombs et autres ornements de l'horloge sur place à partir de l'été 1891. La reprise des cadrans se prolongea ensuite jusqu'à la fin de l'été 1892 et celle des bas-reliefs jusqu'en juin 1893, ce qui laisse à penser que Durand a pu revenir une ou deux fois durant cette période. On voit notamment sur les images après restauration que les clichés ont été pris après la dorure des décors mais avant les ouvrages de peinture décorative.

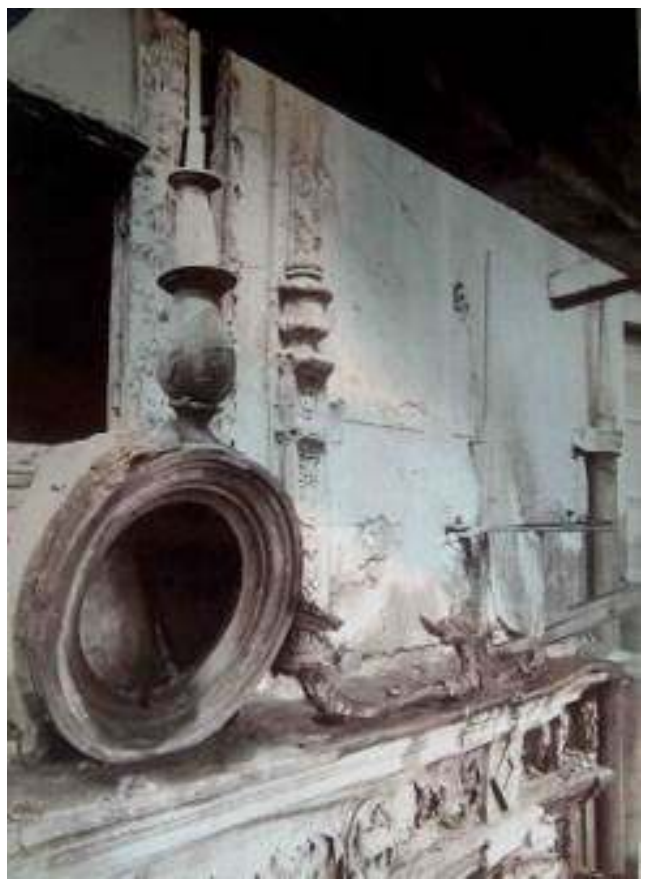

Fig. 5 : Le couronnement du cadran de l'horloge avant restauration, photographie, Charenton-le-Pont, Médiathèque de l'architecture et du patrimoine, album Durand. 


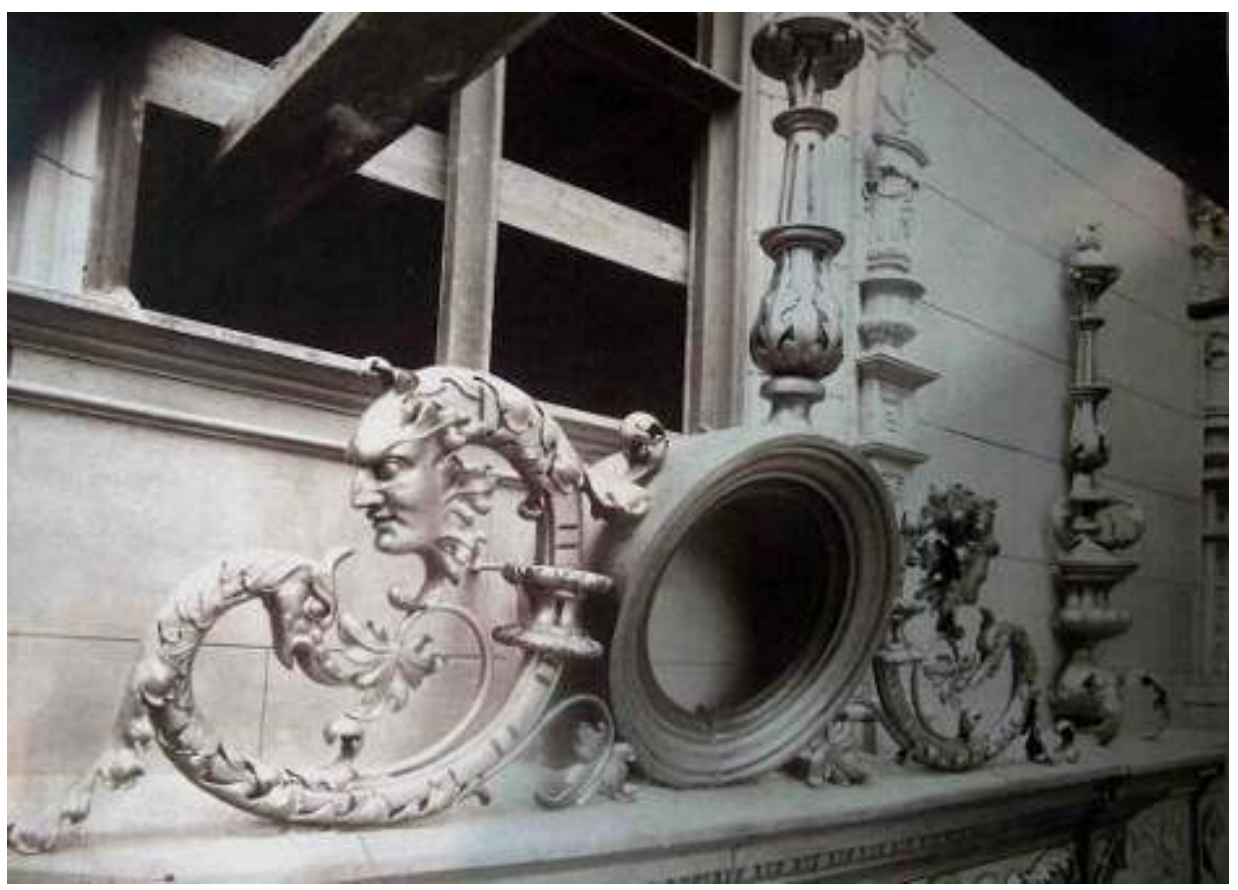

Fig. 6 : Le couronnement du cadran de l'horloge après restauration, photographie, Charenton-le-Pont, Médiathèque de l'architecture et du patrimoine, album Durand.

L'album réalisé par Durand témoigne de la démarche et des objectifs poursuivis par l'architecte évoqués précédemment, c'est-à-dire de la reprise et de la mise en valeur des détails d'ornementation. De fait, c'est bien sûr la partie décorative et les cadrans que se concentra la campagne photographique. Sur l'ensemble des clichés, quatre seulement nous montrent des vues d'ensemble du monument avant et après restauration. Celles-ci sont prises de chaque côté (depuis la rue ou bien en étage dans un immeuble), d'angle ou de face. Concernant les opérations de reprise générale du bâtiment à cheval sur la rue, de la boutique et de sa loggia, aucune étape n'est visuellement documentée, bien que d'importants aménagements aient été effectués. Il est sans doute plus surprenant de constater qu'aucune photographie n'illustre la réfection de l'imposante toiture. Il s'agit pourtant bien d'un choix puisque celle-ci était achevée et en place au moment où Durand est venu à Rouen, car elle apparaît dans les vues générales après restauration. Ce sont donc les ornements restitués, ceux des cadrans en plomb et les diverses parties de la décoration en plâtre recouvrant le pan de bois des deux façades du pavillon du Gros-Horloge, qui figurent dans l'album.

Les photographies illustrant cette partie du chantier ont plusieurs fonctions. Elles ont bien évidemment une valeur documentaire, car elles permettent de suivre l'état avant et après l'intervention à travers des vues d'ensemble et de détails, prises de face ou de trois-quarts, choix très probablement dictés par Sauvageot lui-même. En dehors de ces directives globales, Durand a de toute évidence bénéficié d'une grande liberté pour la réalisation des clichés : les choix des cadrages, le travail sur la lumière qui joue des reflets et permet d'accentuer certains volumes, attestent d'un parti esthétique qui fait de cet album un ensemble de grande qualité et d'une grande force visuelle. Ces photographies permettent aussi de justifier des choix de restauration proposés par l'architecte qui évoluent au fil des observations et des découvertes archéologiques menées in situ. Elles forment ainsi une sorte de colonne vertébrale à partir de laquelle Sauvageot élabore, nourrit et justifie son projet. De ce point de vue, elles attestent de 
son approche respectueuse puisqu'il aborde le Gros-Horloge comme un «lieu de mémoire " sans que cela soit une contrainte, nous l'avons vu. Ainsi, il modifia certains détails comme les rinceaux de la frise au-dessus de la corniche des cadrans, dont le sens fut curieusement modifié. À l'inverse, les vues nous montrent que l'intervention a été particulièrement respectueuse en certains points : il apparaît par exemple, que les basreliefs des roues n'ont pas été entièrement refaits et qu'une partie seulement des arêtes et des ciselures a été reprise pour redonner de la vigueur au trait. Les imperfections subsistantes en sont la preuve. Certains morceaux ont cependant été moins habilement repris, notamment celui consacré à Vénus (Vendredi) qui montre des différences notables dans les détails des personnages et dans le crucifix sur le char.

L'usage de la photographie dans le cas de la restauration du Gros-Horloge de Rouen menée sous la direction de Sauvageot à la fin du XIx siècle, est d'une grande modernité et sans précédent dans sa carrière. Certes, celui-ci a très tôt utilisé de manière systématique la photographie pour illustrer ses projets de restauration ${ }^{41}$. Mais, tout au plus s'agissait-il d'intégrer une ou plusieurs images montrant l'état de dégradation de l'édifice. Ici, l'ambition est autre et le fait que l'intervention d'un photographe soit programmée dès le premier devis des travaux pour documenter la campagne - au moins pour les parties jugées les plus intéressantes - atteste de la démarche scientifique et respectueuse revendiquée par l'architecte. En effet, les enjeux sont d'autant plus importants que l'intervention porte sur l'un des monuments emblématiques de la Renaissance française et qu'il s'agit pour lui d'asseoir un peu plus sa réputation au sein du service des Monuments historiques.

\section{NOTES}

1. «Le Gros-Horloge », La Normandie monumentale et pittoresque, Le Havre, Lemale et Cie, 1894, p. 25.

2. Voir Françoise Boudon, «Le regard du XIX siècle sur le $\mathrm{XVI}^{\mathrm{e}}$ siècle français : ce qu'ont vu les revues d'architecture ", Revue de l'Art, n 89, 1990, p. 39-56.

3. Voir Sophie Nasi, Louis Sauvageot (1842-1908), architecte et restaurateur à Rouen, Rennes / Rouen, PUR / PURH, 2010. Il s'agit de la publication sous une forme remaniée d'une thèse intitulée : Un architecte et une ville à la fin du XIX ${ }^{e}$ siècle: Louis Charles Sauvageot (1842-1908) à Rouen. Constructions. Restaurations, thèse de doctorat d'État sous la direction de Françoise Hamon, université de Paris IV, 2007. Concernant le chantier de restauration du Gros-Horloge, voir également du même auteur: «Une restauration emblématique : le Gros-Horloge de Rouen (1889-1893) », Histoire de l'Art, no 56, avril 2005, p. 53-64.

4. Il avait restauré notamment l'église Saint-Jacques de Dieppe (1875), l'église d'Eu (1876) et la flèche de Caudebec-en-caux (1878). À Rouen, il était intervenu sur l'édicule de la Fierte de SaintRomain et sur les principales églises de la ville (Saint-Maclou, Saint-Vincent, la cathédrale NotreDame...).

5. Voir Alfred Cerné, Les anciens hôtels de ville, leurs beffrois et la grosse horloge, Rouen, Lestringant, 1934, p. 43-54. 
6. Voir L.-R. Delsalle, «Le décor de l'arcade du Gros-Horloge, Contribution à l'interprétation ", Bulletin des Amis des monuments rouennais, octobre 2002-septembre 2003, p. 85-91.

7. Parmi les nombreux travaux rouennais de l'architecte et sculpteur Jean-Pierre Defrance (1694-1768), on peut citer les chœurs des églises de Saint-Maclou (1727) et de Saint-Vincent (1735). Architecte de l'abbaye de Saint-Ouen, on lui doit le plan général des bâtiments conventuels du XVIII ${ }^{\mathrm{e}}$ siècle complété ensuite par Le Brument.

8. Voir Eustache de La Quérière, Notice historique et descriptive sur l'ancien hôtel-de-ville, le Beffroi et la Grosse-Horloge de Rouen, Paris, Aubry, Morel et Cie, Rouen, Herpin, 1864 ; Jules Adeline, op. cit. ; Georges Dubosc, Rouen monumental au XVII et XVIII siècle, ..., Rouen, Cagniard, 1897 ; A. Cerné, op. cit.

9. Le beffroi ne fut classé qu'en 1930 ce qui montre le peu d'intérêt que lui accordait la commission des Monuments historiques, contrairement aux autres éléments du Gros-Horloge.

10. Aubin-Louis Millin, Antiquités nationales ou recueil des monuments..., t. 3, Paris, M. Drocehin, 1791.

11. Négociant et juge consulaire, Eustache de La Quérière (1783-1867) voua sa vie à l'archéologie et à la défense du Vieux Rouen. On lui doit une soixantaine d'études sur la ville. Son ouvrage le plus connu, Description historique des maisons de Rouen les plus remarquables par leur décoration extérieure et par leur ancienneté, illustré par E.-H. Langlois, parut en 1821. Il fut membre de l'Académie de Rouen, de la société des antiquaires de France, de la commission départementale des Antiquités et de bien d'autres sociétés savantes.

12. Robert-Louis Hainaut, Notice historique sur la Grosse-Horloge de Rouen. Son antiquité et sa remarquable conservation, Rouen, Cagniard, 1887.

13. Jules Touzet : architecte municipal de Rouen de 1882 à 1889 . On lui doit notamment quelques écoles, l'abattoir et le marché aux bestiaux.

14. Inspirée de la société des Amis des monuments parisiens de Charles Normand, la société rouennaise fut fondée le 8 juillet 1886 dans le but de défendre le vieux Rouen. Particulièrement influente, elle réunit les élites culturelles de la ville. On lui doit le sauvetage de nombreux édifices comme le «vieux logis" de la rue Saint-Romain à la fin du xix siècle. Voir «Églises, hôtels, vieilles maisons de Rouen», Bulletin des Amis des monuments rouennais (numéro spécial du Centenaire), 1986.

15. Elle réunissait l'adjoint au maire Achille Lefort, les conseillers municipaux Garreta et Garnier, l'horloger Hénault, des érudits (Alfred Darcel, Charles de Beaurepaire, Gaston Le Breton, Maillet du Boullay, Georges Dubosc) et des architectes (Henri Gosselin, Louis Sauvageot, Lucien Lefort, Eugène Fauquet et Jules Touzet), pour la plupart membres de la commission départementale des Antiquités.

16. Lettre au directeur des Beaux-arts le 23 février 1890 (Charenton-le-Pont, Médiathèque de l'architecture et du patrimoine, 81/76/283/149).

17. Louis Ricard (1839-1921) est sans doute la personnalité la plus marquante du Rouen républicain (maire de 1881 à 1886) puisqu'il fut l'un des rares représentants de la région à atteindre une envergure nationale, en devenant deux fois ministres. Sur le plan local, on retient surtout son sectarisme anticlérical. Voir Jean-Pierre Chaline et Anne-Marie Sohn, Dictionnaire des parlementaires de Haute-Normandie 1871-1940, Rouen, Publications de l'Université de Rouen, 2000.

18. Entre autres travaux, nous retiendrons la poursuite de l'assainissement du quartier populaire dit de Martainville, la construction d'une dizaine d'écoles, l'agrandissement du Lycée et de plusieurs bâtiments d'enseignement supérieur.

19. Entré au service des Monuments historiques en 1868, il fut nommé Architecte en chef en 1872. Attaché à la commission l'année suivante, il en devint membre en 1887. Il démissionna lors de l'interdiction de cumul des fonctions en 1892. En 1897, il fut nommé Architecte en chef chargé de l'Ain, de la Saône-et-Loire, de la Loire, du Rhône et du Jura et des arrondissements de Beaune et de Compiègne. Entré au service des Édifices Diocésains en 1880, il fut chargé du diocèse de 
Rouen en 1880, puis de Nantes (1884) et enfin de Beauvais (1886). De 1896 à 1902, il occupa la fonction d'Inspecteur général adjoint avant d'être nommé en 1904, Inspecteur général des Édifices Diocésains en remplacement de Corroyer. La région sud-ouest lui fut confiée.

20. La dépense autorisée s'élevait à 58 226,25 F (réduites à $49483,13 \mathrm{~F}$ ), ainsi répartie : $50 \%$ sur les fonds des Monuments historiques (arrêté du 26/02/1890) ; $16 \%$ sur les fonds du département (délibération du Conseil général du 15/04/1890) et $34 \%$ sur les fonds de la commune (délibération du Conseil municipal du 25/04/1890).

21. Entreprises chargées des travaux : Requier, Rouen (maçonnerie et charpente); Monduit fils, Paris (plomberie); Bonet, Rouen (sculpture et moulages); Lucien Lavigne, Paris (peinture décorative) ; Albert Legrip, Rouen (dorure) ; Château père et fils (horlogerie) ; Petit aîné, Rouen (peinture ordinaire); Boulanger, Rouen (vitrerie d'art).

22. Nommé Inspecteur des travaux des Édifices diocésains de Rouen le 25 février 1882 sous la direction de Sauvageot, Henri Auguste Gosselin (1844-1902) fut également Inspecteur des travaux de restauration du Palais de justice et de la Fierte de Saint-Romain. Entres autres travaux personnels, on lui doit la restauration du chœur de l'église Saint-Nicaise à Rouen, la construction des sacristies des églises d'Harfleur et de Charleval, celle du clocher de l'église du Mont-auxMalades de Rouen. Enfin, il restaura en 1891 l'hôtel du Bourgtheroulde, d'après le projet de Sauvageot.

23. Restauration du Gros Horloge à Rouen, rapport de l'architecte Louis Sauvageot, 15 novembre 1889 (Charenton-le-Pont, Médiathèque de l'architecture et du patrimoine, 81/76/283/149).

24. Sur la voûte, Sauvageot se contenta de procéder à un rejointoiement général pour assurer sa pérennité. Bien que des traces de peinture et de dorure aient été identifiées, l'architecte n'évoqua jamais la possibilité de recréer le décor sur cette partie.

25. Perçue comme un monument charmant mais sans réelle valeur, la fontaine était pourtant très altérée par l'écoulement des eaux pluviales du beffroi. En 1894, une chute de pierres endommagea la cuisse de la figure féminine : Sauvageot limita l'intervention et la partie atteinte fut nettoyée puis enduite de cire chaude et colorée. La fontaine, victime des pluies acides, continua de se dégrader. Dans les années 1930, les parties les plus atteintes furent déposées à l'abri et remplacés par des copies (Charenton-le-Pont, Médiathèque de l'architecture et du patrimoine, 81/76/283/149).

26. Rapport dressé par l'Inspecteur général Selmersheim, séance de la commission du 7 février 1890 (Charenton-le-Pont, Médiathèque de l'architecture et du patrimoine, 81/76/283/149).

27. Lettre d'Achille Lefort, adjoint au Maire de Rouen au Ministre de l'Instruction publique et des Beaux-arts, le 23 avril 1889 (Charenton-le-Pont, Médiathèque de l'architecture et du patrimoine, 81/76/283/149).

28. «Deux des pilastres de l'étage supérieur sont presque complets; la saillie de l'un des pilastres de l'étage intermédiaire est encore apparente sur la façade à l'est. Du même côté, il existe, à la gauche du haut du cadran, toute une partie d'entablement [...]; les amorces du même entablement se retrouvent à la droite du cadran. Le tracé d'appareil sur les fonds unis existe encore sur une assez grande surface." (Restauration du Gros-Horloge à Rouen, rapport de l'architecte Louis Sauvageot, 15 novembre 1889, Charenton-le-Pont, Médiathèque de l'architecture et du patrimoine, 81/76/283/149).

29. Cet hôtel situé rue des Carmes, bâti pour Nicolas Romé, portait la date de 1525. Racheté en 1589 par la Cour des comptes, il fut agrandi aux XVII ${ }^{\mathrm{e}}$ et XVIII ${ }^{\mathrm{e}}$ siècles. Presque entièrement détruit lors des bombardements de juin 1944, il ne subsiste de cet hôtel que des vestiges de la façade conservés sur place. Voir I. Letteron, «Les hôtels de Rouen à la Renaissance », L'architecture de la Renaissance en Normandie, t. II, Condé-sur-Noireau / Caen, Éditions Charles Corlet / Presses universitaires de Caen, 2003, p. 115-119.

30. Sauvageot, dans son rapport du 15 novembre 1889, souligne la particularité du décor du pavillon: «Le fait d'une décoration importante extérieure exécutée en plâtre au XVI ${ }^{\mathrm{e}}$ siècle, est, 
croyons-nous, très rare. On connaît de nombreux exemples de cet emploi du plâtre à la Renaissance dans les décorations intérieures mais non au dehors. » (Charenton-le-Pont, Médiathèque de l'architecture et du patrimoine, 81/76/283/149).

31. La question des finitions souleva un débat au sein de la commission des Monuments historiques : fallait-il laisser les nouvelles peintures et dorures en l'état ou atténuer leur aspect neuf et trop brillant par le recours à un artifice ? Le choix fut laissé à Sauvageot (P. V. de la visite de la commission des Monuments historiques à Rouen le 13 novembre 1891, Charenton-le-Pont, Médiathèque de l'architecture et du patrimoine, 80/15/10).

32. Ibid., séance du 22 février 1890 (Charenton-le-Pont, Médiathèque de l'architecture et du patrimoine, 80/15/10).

33. Rapport dressé par Sauvageot le 4 octobre 1892 portant d'une part sur les travaux d'horlogerie et d'autre part, sur la description des sujets représentés sur les deux grandes roues mobiles (Charenton-le-Pont, Médiathèque de l'architecture et du patrimoine, 81/76/283/149).

34. Jannie Mayer, «Les épis de faitage et les plombs de couverture », Bulletin de l'ICOMOS, $\mathrm{n}^{\circ}$ 40-41, 1997, p. 22-27.

35. Eustache de La Quérière, Essai sur les girouettes, épis, crêtes et autres décorations des anciens combles et pignons, ..., Paris, Derache, 1846, p. 7-8.

36. Colette di Matteo, "Les ateliers Monduit ", Le Mont-Saint-Michel, l'archange, la flèche, Paris, Caisse nationale des Monuments historiques, 1987, p. 129.

37. Gros-Horloge de Rouen par Durand, s. d., 28 planches photographiques en noir et blanc, $44 \mathrm{~cm} \mathrm{x}$ $31 \mathrm{~cm}$ (Charenton-le-Pont, Médiathèque de l'architecture et du patrimoine, Fo E 244).

38. Charenton-le-Pont, Médiathèque de l'architecture et du patrimoine, 1996/089: relevés de peintures murales, Gros-Horloge, décoration des zodiaques, Sauvageot, 1891.

39. Restauration du Gros-Horloge à Rouen, rapport de l'architecte Louis Sauvageot, 15 novembre 1889 (Charenton-le-Pont, Médiathèque de l'architecture et du patrimoine, 81/76/283/149).

40. Les clichés ont également été déposés au service des Monuments historiques et rangés à part.

41. Dès la fin des années 1860 , la présentation des rapports de restauration de Sauvageot devient systématique, avec des vues photographiques jointes ou collées sur la première page, en haut à gauche.

\section{AUTEUR}

\section{SOPHIE NASI}

Institut Catholique de Paris 


\title{
Du document d'étude à l'image illustrative
}

\author{
L'usage de la photographie par des architectes
}

\section{Florent Miane}

À partir des années 1850, la photographie d'architecture apparait dans de nombreuses archives publiques ou privées. Elle est en grande partie créée par les services municipaux et les constructeurs (architectes, ingénieurs, entrepreneurs...). Si cette pratique est au départ la marque d'une certaine culture, celle des architectures historicistes ${ }^{1}$ et des ingénieurs de l'École des ponts et chaussées ${ }^{2}$, elle se généralise rapidement et devient omniprésente sur les chantiers de construction dans les années 1880.

Afin d'aborder l'usage de l'imagerie photographique produite par les architectes, nous proposons de l'étudier par rapport à la transformation de la ville de Bordeaux au xIX siècle (1860-1890). C'est-à-dire de la resituer, dans une vision globale de la gestion de la ville, afin de saisir son apparition, ses usages et ses lectures. La production d'images par les architectes dépend, en effet, tout autant des méthodes de travail que des relations qu'ils entretiennent avec les décideurs (commanditaires, conseil municipal...) et les exécutants (entrepreneurs, services administratifs...). Dans ce contexte, le croisement des archives écrites et figurées renseigne sur le moment où ces images apparaissent, les discours dont elles sont l'illustration, leurs réseaux de diffusion et l'influence progressive d'autres imageries telles que celles produites par les ingénieurs.

Les archives de la ville de Bordeaux permettent d'étudier précisément ce phénomène. Elles conservent plus d'un millier de photographies d'architecture en ce qui concerne le $\mathrm{XIX}^{\mathrm{e}}$ siècle. La plupart de ces images peuvent être rattachées à la production des constructeurs c'est-à-dire à la production de documents administratifs. Il s'agit donc d'une mémoire partielle et partiale qui rend compte non pas de la production des photographes mais des relations entre les différents acteurs de la ville. Ainsi, à Bordeaux, la photographie d'architecture est foisonnante alors que le portrait est peu présent, bien qu'il soit la principale source de revenus des photographes à cette époque. 
Les usages de la photographie par les architectes peuvent être répartis dans deux grandes périodes qui seront étudiées successivement. La première est celle de l'apparition de cette imagerie qui se fait dans le contexte de l'architecture historiciste de Paul Abadie lors des travaux de restauration qu'il entreprend à la fin des années 1850. La seconde, liée à l'architecture éclectique, est marquée par la diversification des usages visible à l'occasion de la construction des facultés par les architectes Jean-Louis Pascal et Charles Durand dans les années 1880.

Il est à noter que, si Bordeaux participe comme d'autres villes aux habituelles transformations urbaines de la deuxième moitié du XIXe siècle, cette ville présente une particularité. Si à Lille, Lyon, Marseille ou Paris, les commandes sont principalement réalisées par des photographes nés dans les années 1810-1820 (Alphonse Le Blondel né en 1814, Louis Froissart né en 1815, Adolphe Terris né en 1820, Charles Marville né en 1813 et Auguste Collard né en 1812), à Bordeaux, cette imagerie est principalement réalisée par un photographe de la génération suivante, Alphonse Terpereau, né en 1839 , qui a su faire de cette ville des images novatrices d'une grande qualité visuelle ${ }^{3}$.

\section{La restauration de l'église Sainte-Croix}

Paul Abadie est le premier architecte qui ait fait usage de photographies à Bordeaux et dont les commandes ont été conservées. Ces images se trouvent aujourd'hui dans les archives de la ville et dans ses archives personnelles déposées à la Bibliothèque Nationale. Elles ont été réalisées au cours de deux chantiers de restauration : celui de l'église Sainte-Croix et celui de la tour Saint-Michel et répondent à deux usages, scientifique et illustratif. Cette utilisation dépend d'une certaine tradition, l'architecture historiciste, mise en place par Duban lors de la restauration du château de Blois puis par Lassus et Viollet-le-Duc sur le chantier de la cathédrale Notre-Dame à Paris où Abadie fut nommé inspecteur des travaux. À son arrivée à Bordeaux en tant qu'architecte diocésain, il utilise donc une technique graphique qui est à la fois un outil de travail et la marque d'une appartenance à un mouvemment architectural. De son côté, le conseil municipal va créer tout au long des travaux des documents administratifs parmi lesquels figurent des photographies.

Celles réalisées lors de la restauration de l'église Sainte-Croix permettent de saisir les principaux usages qu'en fait Abadie. Les travaux, commandés par la fabrique, consistent à construire un nouveau clocher afin de remédier au désagrément que cause l'unique clocher sud contigu au dortoir d'un hospice. Pour le conseil municipal, qui participe au financement des travaux, il s'agit d'aligner l'édifice sur la voirie, de créer une façade symétrique et de combler un vide sans cesse rempli d'ordures. La réalisation du projet est confiée à Abadie. Il comprend la construction du clocher et la consolidation de la façade ${ }^{4}$.

Les premières photographies à être produites dans ce contexte ont un usage administratif. Il s'agit pour le conseil municipal de pouvoir contrôler le travail de l'architecte. La restauration d'un édifice classé Monument historique dès 1840 suscite en effet des craintes de la part de certains conseillers municipaux ${ }^{5}$. Le maire décide donc de créer une commission spéciale venant épauler la Commission des monuments historiques et de faire des photographies avant travaux qui puissent valider la pertinence du projet et garantir l'intégrité de l'édifice. Ces images sont mentionnées à 
deux reprises dans les délibérations du conseil municipal et lors de la rédaction du devis :

«Art. 3 - Pour assurer la rigoureuse conservation du style de l'église, il sera fait, avant toute œuvre, une photographie de la façade actuelle et une commission spéciale sera nommée pour surveiller l'exécution des travaux ${ }^{6}$.»

« Dépenses relatives aux renseignements artistiques commandés par l'architecte et reconnus par lui indispensables. [...] Compte Poirier - photographies de l'état ancien - une vue d'ensemble - deux photographies de détails - en tout trois clichés $240 \mathrm{~F}^{7}$.»

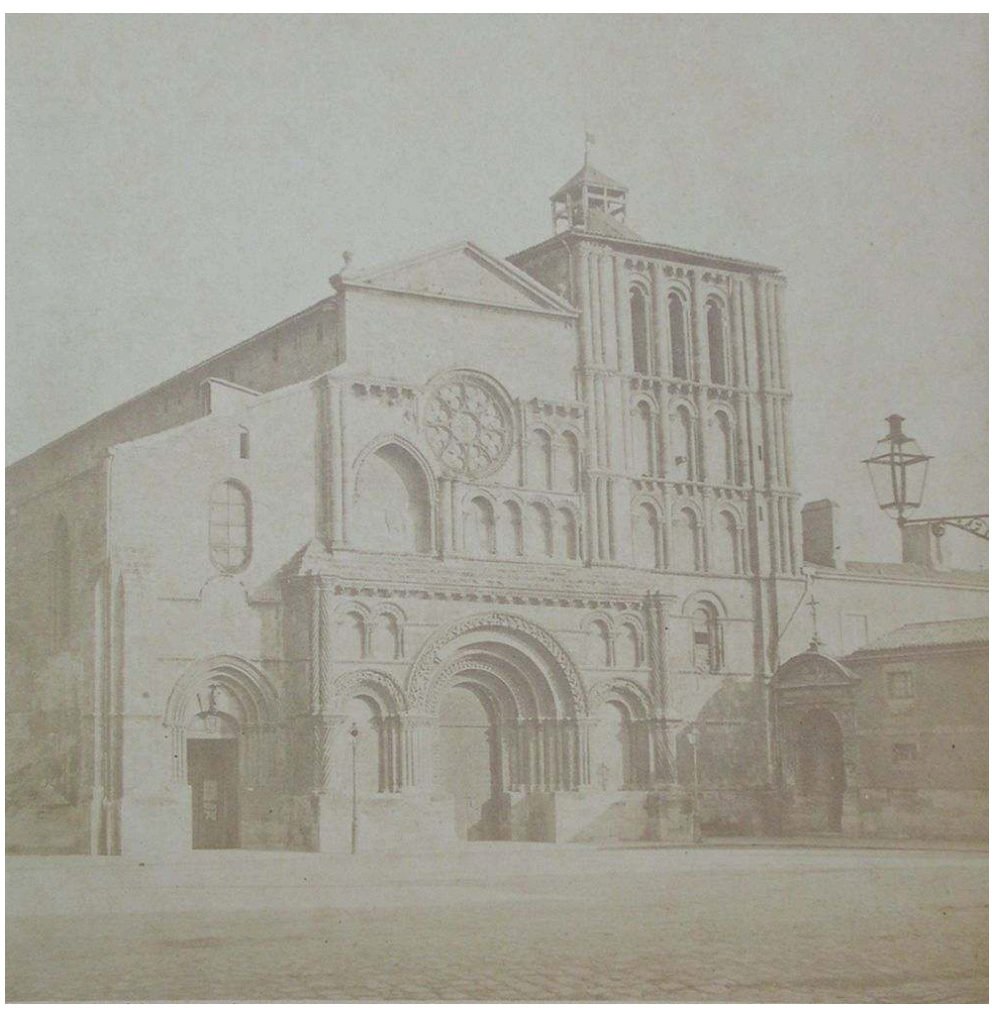

Fig. 1 : Alphonse Terpereau, Église Sainte-Croix avant restauration, vers 1860, tirage sur papier albuminé, $19,9 \times 19,8 \mathrm{~cm}$, Bordeaux, archives municipales, 4 I 89.

La photographie est utilisée, dans ce cas, pour apaiser les inquiétudes de certains conseillers municipaux et permettre le vote en faveur des travaux. Il ne reste aujourd'hui de cette commande qu'une image (fig.1) conservée dans le fonds iconographique des archives municipales ${ }^{8}$. Le $1^{\text {er }}$ mai 1862 , le nouveau clocher s'élève à sept mètres au-dessus du sol'. À cette occasion, l'entrepreneur Danjou fait faire quarante photographies ${ }^{10}$.

« Restauration de la façade de l'église $\mathrm{S}^{\text {te }}$ Croix. / Récapitulation des dépenses. / [...]

/ De Parada 40 photographies 50,50 F / Exercice 1862. »

Ces images ont aujourd'hui disparu. Elles permettaient de juger du travail de l'architecte, mais aussi de démontrer le savoir-faire de l'entrepreneur auprès des différents financiers et des futurs commanditaires.

Les observations faites par Abadie lors de la consolidation de la façade vont lui permettre de réaliser un deuxième projet qui va modifier la lecture de la vue avant travaux. Ce nouveau projet consiste à restituer le décor roman en grande partie disparu. Souhaitant l'imposer, il imagine un édifice idéal débarrassé des modifications gothiques et classiques ${ }^{11}$. Il parvient à convaincre la commission de surveillance du 
bien-fondé de ses observations et de ses restitutions ${ }^{12}$. La commission convainc à son tour le maire, qui voit dans la réalisation d'un monument ambitieux la possibilité de récupérer les voix d'un quartier populaire. Dès lors, pour la majorité du conseil municipal, la photographie n'est plus l'image précieuse d'un état ancien. Elle n'est que le reflet d'un édifice aux apparences trompeuses dont l'architecte a fait tomber le masque ${ }^{13}$.

Les nouveaux travaux vont conduire Abadie à utiliser la photographie. Ils consistent à compléter les arcatures romanes, créer un tympan sculpté (le Christ en gloire, deux anges, le tétramorphe), construire un lanternon poitevin, déplacer l'arc ogival, supprimer la rose gothique et détruire le fronton classique, placer des hauts-reliefs dans les arcades (la Vierge, les quatre évangélistes, les apôtres) et installer des sculptures dans l'arc (un cavalier, un dragon, une dame). Ce vaste programme implique la mise à disposition de nombreux modèles pour réaliser la statuaire. En effet, le 10 août 1864, Abadie propose la candidature du sculpteur Pascal, qu'il présente comme le plus capable d'exécuter la statuaire $\mathrm{du} \mathrm{xII}^{\mathrm{e}}$ siècle ${ }^{14}$. Cependant, la nouveauté des références médiévales qui ne font plus intervenir les modèles classiques nécessite un apprentissage particulier. Abadie, qui ne croit pas à la capacité des sculpteurs à se former par le dessin, exige que les modèles soient observés in situ ou reproduits par des techniques d'empreintes ${ }^{15}$. Il a recours au voyage et à la production de moulages et de photographies :

« Dépenses relatives aux renseignements artistiques commandés par l'architecte et reconnus par lui indispensables. / Compte Poirier - photographies de l'état ancien une vue d'ensemble - deux photographies de détails - en tout trois clichés $240 \mathrm{~F}$. / Compte Marville - photographies du St Georges de Châteauneuf et de la façade de la cathédrale d'Angoulême - Trois clichés - et voyages 500 F. / Estampage du cavalier de l'île $S^{t}$ Georges - près Bordeaux (compte Gaty) 86,50 F. / Estampage à Féniaux (compte Vidiani) $152 \mathrm{~F}^{16}$.»

«M. Abadie a fait un voyage à Moissac (Tarn-et-Garonne) à Autun et à Sens. / M. Pascal a visité - d'après l'ordre de M. Abadie, Ruffec, Civray, Parthenay, Châteauneuf, Cognac, Moissac et Toulouse. / Moi-même [Lambert, directeur des travaux], je suis allé à Saintes, Féniaux, Aulnay, Surgères et Poitiers ${ }^{17}$. "

La photographie est dans ce cas une image fidèle dont le but est l'acquisition d'un savoir-faire. L'utilisation des reproductions mécaniques est déterminée par la volonté de substituer l'inspiration du sculpteur au modèle choisi par l'architecte. Ces photographies ne sont plus des documents administratifs (contrôle) mais des documents qui trasmettent un savoir (modèle). La nouveauté des méthodes de travail utilisées par l'architecte implique un aménagement du circuit financier. Alors que le modèle est habituellement considéré comme faisant partie de l'œuvre du sculpteur, les reproductions automatiques font partie des techniques de l'architecte et sont payées par ce dernier ${ }^{18}$. 


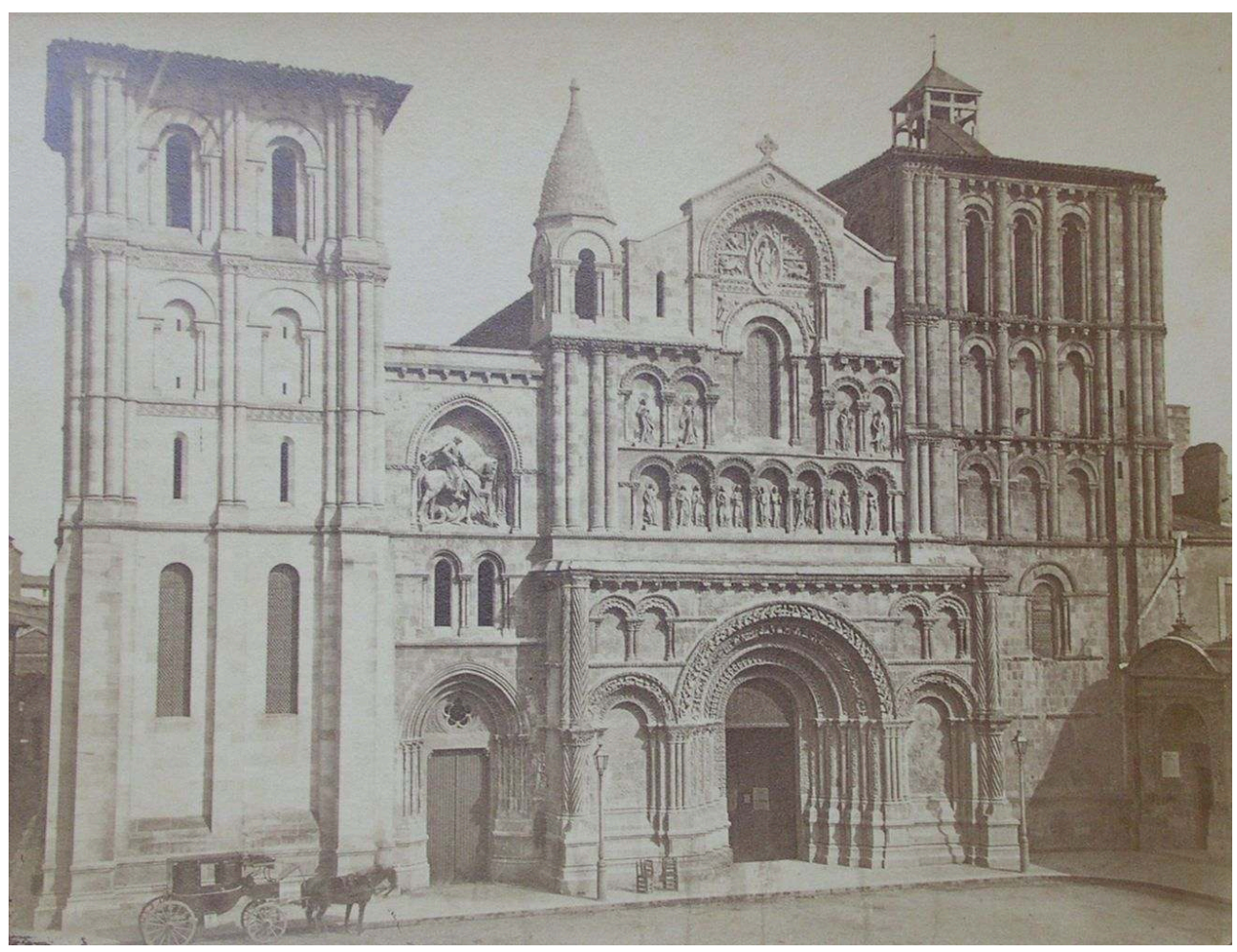

Fig. 2 : Alphonse Terpereau, Église Sainte-Croix après restauration, vers 1865, tirage sur papier albuminé, 21,8 × 28,9 cm, Bordeaux, archives municipales, 4 I 10 .

Une vue de l'édifice après restauration est réalisée par le photographe Alphonse Terpereau (fig. 2). Destinée au grand public, elle se retrouve aux archives municipales ${ }^{19}$ et dans le dépôt légal du photographe à la Bibliothèque Nationale ${ }^{20}$. Cette photographie est collée sur bristol et encadrée d'une zone plus foncée imitant la cuvette de la gravure sur cuivre dont elle reprend les conventions pour la signature ("Terpereau Phot.» signifiant photographe) ${ }^{21}$. Rangée dans un protefeuille ou encadrée sur un mur, elle devient peu à peu un objet de décoration qui participe aux stratégies d'ameublement des intérieurs bourgeois ${ }^{22}$.

Elle se retrouve par ailleurs dans l'album photographique qu'Abadie fait assembler lors de sa réception à l'Académie française ${ }^{23}$ et qui montre ses principales réalisations. Les restaurations sont montrées selon le même principe : la vue avant travaux, anonyme, proche du dessin technique (plein cadre, frontalité) précède les vues de l'édifice achevé mis en scène par des photographes renommés. Cette réthorique sert alors à valoriser le travail de l'architecte. La photographie remplit ici un rôle démonstratif.

\section{La reconstruction de la tour Saint-Michel}

La restauration de la flèche Saint-Michel, réalisée par Abadie parallèlement à celle de l'église Sainte-Croix, permet d'étudier les autres usages de la photographie dans le contexte de son apparition à Bordeaux. Si l'on retrouve, sur ce chantier, certains usages évoqués à Sainte-Croix, d'autres s'imposent en raison du gigantisme des travaux. La tour Saint-Michel sera en effet après sa restauration l'une des plus hautes tours de style gothique (cent quatorze mètres). 
Les travaux consistent, dans un premier temps, à reconstruire la flèche détruite en 1768 lors d'une tempête et replacée depuis par un télégraphe. Il s'agit pour la fabrique de redonner à l'édifice sa fonction première et pour le conseil municipal de doter la ville d'un monument prestigieux qui la symbolise et qui ne manquera pas d'attirer le regard des voyageurs et marquer l'imagination des touristes ${ }^{24}$.

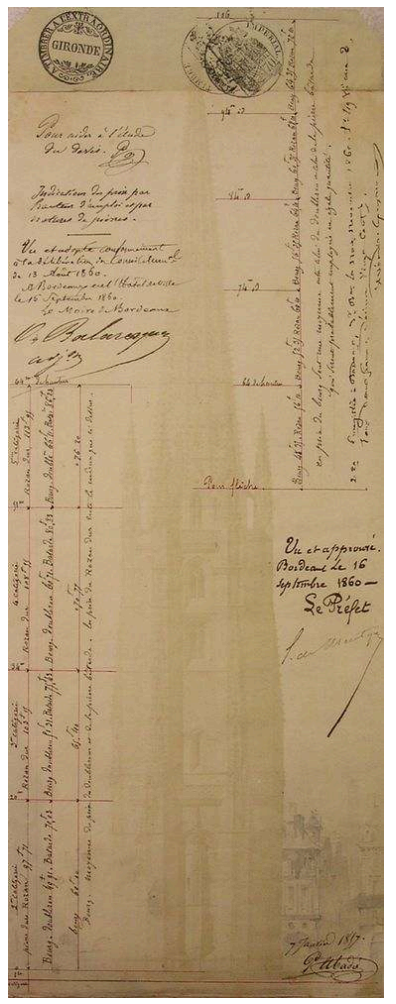

Fig. 3 : Anonyme, Projet de restauration de la tour Saint-Michel, 1857, tirage sur papier albuminé, 36,8 x $13,7 \mathrm{~cm}$, Bordeaux, archives municipales, 3040 M 1

La première photographie réalisée dans ce contexte est anonyme (fig. 3). Elle reproduit un dessin, celui du projet de la tour. Il en existe deux tirages : le premier est intégré au dossier de restauration constitué par l'architecte, comprenant l'histoire de l'édifice, les partis pris de la restauration et le coût des travaux. L'image est datée par l'architecte du 7 novembre $1857^{25}$. L'exactitude et l'automatisme du processus lui donnent une valeur symbolique: elle reçoit les signatures officielles du maire, du préfet et de l'architecte. Elle est aujourd'hui la plus ancienne photographie d'architecture identifiée dans les fonds d'archives publiques de Bordeaux. Ne faisant pas partie du fonds iconographique, elle ne porte pas de cote lui permettant d'être reconnue en tant qu'image $^{26}$. Une autre épreuve, issue du même négatif, toujours anonyme, se trouve dans le même dossier ${ }^{27}$. Isolée et collée sur un bristol blanc, elle est aisément consultable et permet la diffusion du projet parmi les membres du conseil municipal. L'usage de la photographie, dans ce cas, libère l'atelier de l'architecte des travaux de reproduction, le rendant disponible pour d'autres tâches ${ }^{28}$.

Contrairement à Sainte-Croix, la restauration de la tour n'entraîne pas de débat. Une ancienne gravure représentant l'édifice permet à l'architecte de restituer aisément son aspect originel ${ }^{29}$. Malgré quelques modifications apportées par l'architecte, le projet est accepté à l'unanimité (la flèche de quarante mètres est bien plus haute que la précédente ; le décor d'oculus polylobés remplace les flammèches gothiques). 
Avant les travaux, le conseil municipal commande une vue de l'édifice. Les archives de la ville conservent cinq tirages anonymes dans le fonds iconographique ${ }^{30}$. Aucune image ne porte de date, aucun devis n'y fait référence. Cependant, la multiplication des tirages et le lieu de conservation semblent montrer un usage administratif identique à celui de Sainte-Croix. Par ailleurs, le point de vue et le cadrage font référence au dessin technique. La vue est prise du sol mais suffisamment loin de l'édifice pour limiter la contre-plongée, elle cadre étroitement l'édifice laissant à peine deviner le contexte urbain. Cette image permet de contrôler l'exactitude du relevé et de s'assurer du respect des parties anciennes pendant les travaux ${ }^{31}$.

Lors de la construction de la flèche, des fissures apparaissent et se développent dans la maçonnerie de la base. En 1863, le conseil municipal, constatant l'augmentation inquiétante des lézardes, arrête les travaux et nomme une commission de surveillance pour trouver une solution ${ }^{32}$. Cette commission élabore un nouveau projet s'appuyant sur des observations de l'architecte. Il s'agit de renforcer les contreforts extérieurs, de détruire les murs aveugles de la base et de les étrésillonner par une succession d'arcatures ouvertes. Cette solution change radicalement l'apparence de l'édifice mais correspond, comme pour l'église Sainte-Croix, à des observations faites par l'architecte concernant d'anciennes arcatures médiévales dissimulées dans les murs de la base ${ }^{33}$.

Les archives départementales de la Gironde conservent une photographie de Charles Marville reproduisant le second projet de l'architecte ${ }^{34}$. Cette image fait écho à deux lettres émanant du conseil municipal et destinées au préfet. La première mentionne que le projet a été transmis au préfet ${ }^{35}$, l'autre précise qu'un accord de la Commission des monuments historiques permettrait de faciliter le vote en urgence de nouveaux crédits $^{36}$. Cette photographie est un instrument de communication entre le maire et le préfet visant à accélérer et faciliter les démarches administratives.

Pendant les travaux, Abadie n'a pas manqué de demander à Marville des photographies de l'échafaudage recouvrant la tour. Il reste aujourd'hui dans les archives publiques trois de ces vues. Chacune d'elles n'est connue qu'à un seul exemplaire. L'une représentant la tour est conservée aux archives municipales (acquisition par un archiviste ou versement du conseil municipal). La deuxième qui représente la flèche est conservée aux archives départementales (fonds de la Commission des monuments historiques). La troisième, qui montre la base, est conservée à la Bibliothèque Nationale (fonds de l'architecte). La rareté et la répartition de ces photographies semblent montrer qu'elles ont été peu diffusées et qu'elles sont liées à des usages administratif ou privé.

Ces images, reprenant toujours le même principe, sont parfaitement composées. Le photographe a su se placer en hauteur et cadrer de façon à ce que la tour se détache du contexte urbain qui lui sert de fond. Les images se structurent en deux registres superposés d'égale dimension: la ville et l'église, le ciel et la flèche. Dans chacun des registres, les différents éléments sont parfaitement lisibles. 


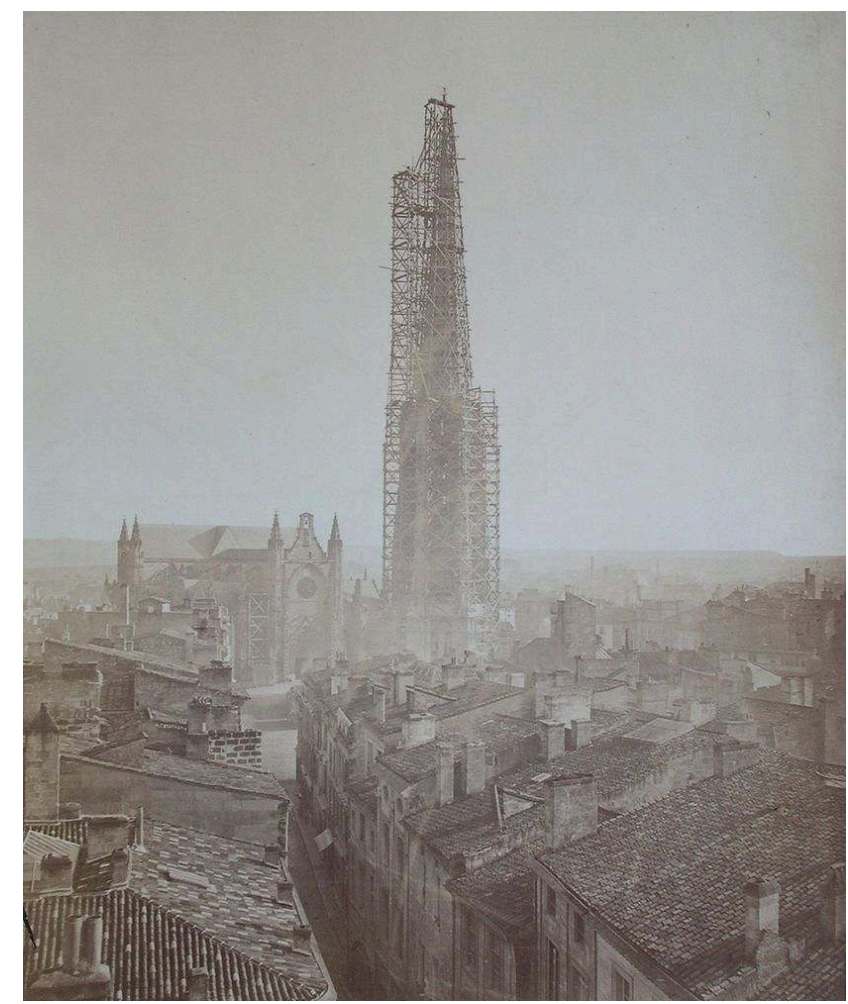

Fig. 4 : Alphonse Terpereau, Échafaudage de la tour Saint-Michel, 1865, tirage sur papier albuminé, 46,6 x $37,9 \mathrm{~cm}$, Bordeaux, bibliothèque municipale, XVI 51-4.

Parallèlement à cette production réduite, les ingénieurs de la ville vont faire de l'échafaudage de la tour le symbole du savoir-faire des services municipaux qui est, cette fois-ci, largement diffusé. Ainsi, en 1865, Louis Lancelin, le directeur du Service des travaux publics de Bordeaux commande à Terpereau une photographie de la tour avant le démontage des échafaudages (fig. 4$)^{37}$. Une lettre indique que l'ingénieur a luimême choisi le cadrage et le point de vue. Ce qu'il obtient correspond à ce qu'il désire : une vue de la partie haute de la flèche recouverte de l'échafaudage se détachant sur un ciel vide. Coupé du contexte urbain, l'échafaudage apparaît ici comme une icône représentant la volonté et la puissance du conseil municipal.

Lancelin commande quarante tirages de cette vue qu'il conserve dans le portefeuille des Travaux publics et qu'il distribue au conseil municipal, à l'architecte, aux entrepreneurs ainsi qu'à la bibliothèque municipale, aux archives municipales et à la Commission des monuments historiques. Les ingénieurs établissent ainsi leur propre culture, s'imposent aux yeux de leurs collaborateurs et produisent les éléments de leur histoire. La répartition actuelle de ces images dans plusieurs fonds d'archives (treize photographies conservées aux archives municipales ${ }^{38}$, à la bibliothèque municipale ${ }^{39} \mathrm{et}$ aux archives départementales ${ }^{40}$ ) reflète encore sa diffusion. Les devis ont été conservés ${ }^{41}$.

Terpereau a cependant outrepassé cette commande en proposant une autre vue de l'échafaudage qu'il destine au grand public (fig. 4$)^{42}$ et qui sera récupérée par l'architecte ${ }^{43}$. Cette image montre la totalité de la tour recouverte par les échafaudages et insérée dans le tissu urbain dont elle est le prolongement. La composition repose sur la fusion des éléments et l'équilibre des masses. Cette vue, qui réintègre la tour dans son contexte, est une création du photographe. Il réalise peu après, selon le même point de vue et le même cadrage, une vue de la tour achevée ${ }^{44}$, qui accompagne une 
photographie de Marville dans l'album de l'Institut de France, selon la même réthorique observée pour l'église Sainte-Croix, afin de valoriser le travail de l'architecte (vue avant travaux frontale et anonyme; vues après travaux mises en scène et signées $)^{45}$.

Le chantier de la tour Saint-Michel montre que l'usage des photographies d'architecture se diversifie en fonction des différents acteurs des travaux. Chacune de ces cultures les utilise selon une tradition qui lui est propre (architectes ou ingénieurs). L'étude de la période suivante pemet de saisir l'évolution et l'interraction de ces différentes imageries ainsi que leurs enjeux.

\section{La construction des facultés de médecine et des sciences}

La période suivante, celle des années 1880-1890, voit l'arrivée d'une autre génération d'architectes qui va répondre à de nouvelles commandes. Parmi celles-ci se trouvent les facultés de médecine et de sciences, conçues respectivement par Jean-Louis Pascal et Charles Durand ${ }^{46}$. Ces constructions, qui sont les plus importantes de cette décennie à Bordeaux et qui prennent le relais des grandes campagnes de restauration de la période pécédente, vont livrer une quantité imporatnte de photographies qui permettent de comprendre l'évolution des usages de ces images. L'intérêt d'étudier ces deux chantiers, très proches dans leur organisation, est de montrer que si la production d'images est assez semblable (thèmes, points de vue...), leurs usages diffèrent en raison du statut des architectes : privé pour Pascal et municipal pour Durand.

La construction des facultés de Bordeaux entre 1880 et 1886 témoigne de la réforme de l'enseignement supérieur entreprise sous la Troisième République. Son organisation résulte d'un nouveau modèle pédagogique prenant modèle sur celui de l'université allemande qui apparaît, après la défaite de 1870, comme le plus moderne et le plus performant. Il se caractérise notamment par l'importance de l'expérimentation et des travaux pratiques visibles par la multiplication des laboratoires et des collections. Ces nouveaux espaces, qui vont profondément modifier les formes des facultés, sont ceux qu'enregistre la photographie.

Lors de la construction de la faculté de médecine par Pascal, trois campagnes photographiques ont été réalisées. Toutes les trois sont commandées par l'architecte. Les deux premières lui permettent de visualiser chaque phase des travaux depuis son atelier situé à Paris. La troisième reproduit les modèles de sculptures en plâtre qu'il

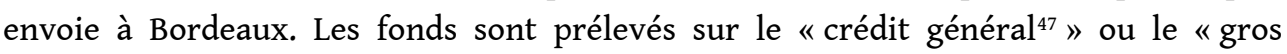
œuvre ${ }^{48} »$ de la faculté.

Les deux premières campagnes sont réalisées par Terpereau. Il reste aujourd'hui vingtquatre photographies conservées aux archives municipales qui correspondent pour moitié à l'achèvement des principales phases des travaux (des fondations aux différents étages) et pour moitié à la phase finale des travaux (intérieur et extérieur). Il ne reste de cette production que les doubles conservés dans les archives de la ville. Ces images comportent une légende avec un titre ${ }^{49}$ et la date précise de la prise de vue ${ }^{50}$. Elles accompagnent les feuilles de métré que l'inspecteur Victor Mialhe destine à l'architecte et ne font que confirmer les descriptions de l'inspecteur qui rendent compte 
précisément de l'avancement du chantier. Lorsqu'elles apparaissent dans la correspondance écrite, ce n'est que pour évoquer des questions de détail :

«M. Terpereau m'a envoyé directement une photographie qui, malheureusement, est bien noire, - probablement parce qu'on ne pouvait pas faire mieux dans un intérieur. J'avais vu sur les marches dans cette épreuve encore des planches et je trouvais que c'était vraiment bien prolonger ce provisoire: votre renseignement, qui m'annonce qu'elles ont été retirées, me fait plaisir ; mais vous me réjouirez tout à fait quand vous me direz que le musée est fini ${ }^{51}$ !! ! !»

Les vues des modèles de sculptures en plâtre ont été réalisées à la demande de l'architecte au cas où l'un d'entre eux, envoyés depuis Paris, subirait des dommages :

«Les modèles des sculptures de la faculté de médecine étant en partie arrivés, Monsieur Pascal me charge de vous demander l'autorisation de les faire photographier, au compte de la ville, ce travail n'ayant pu être fait à Paris, par suite de l'exiguïté du local dans lequel ils ont été exécutés. Monsieur Pascal estime qu'il serait prudent et même utile d'avoir ces reproductions, dans le cas où quelque accident viendrait à nous priver d'un modèle, dont la reconstitution serait longue et dispendieuse $\mathrm{e}^{52}$."

Toutes ces images, qu'elles représentent le chantier ou reproduisent les modèles, témoignent plus de la multiplication des contrôles et du travail à distance qu'elles ne répondent à un réel besoin.

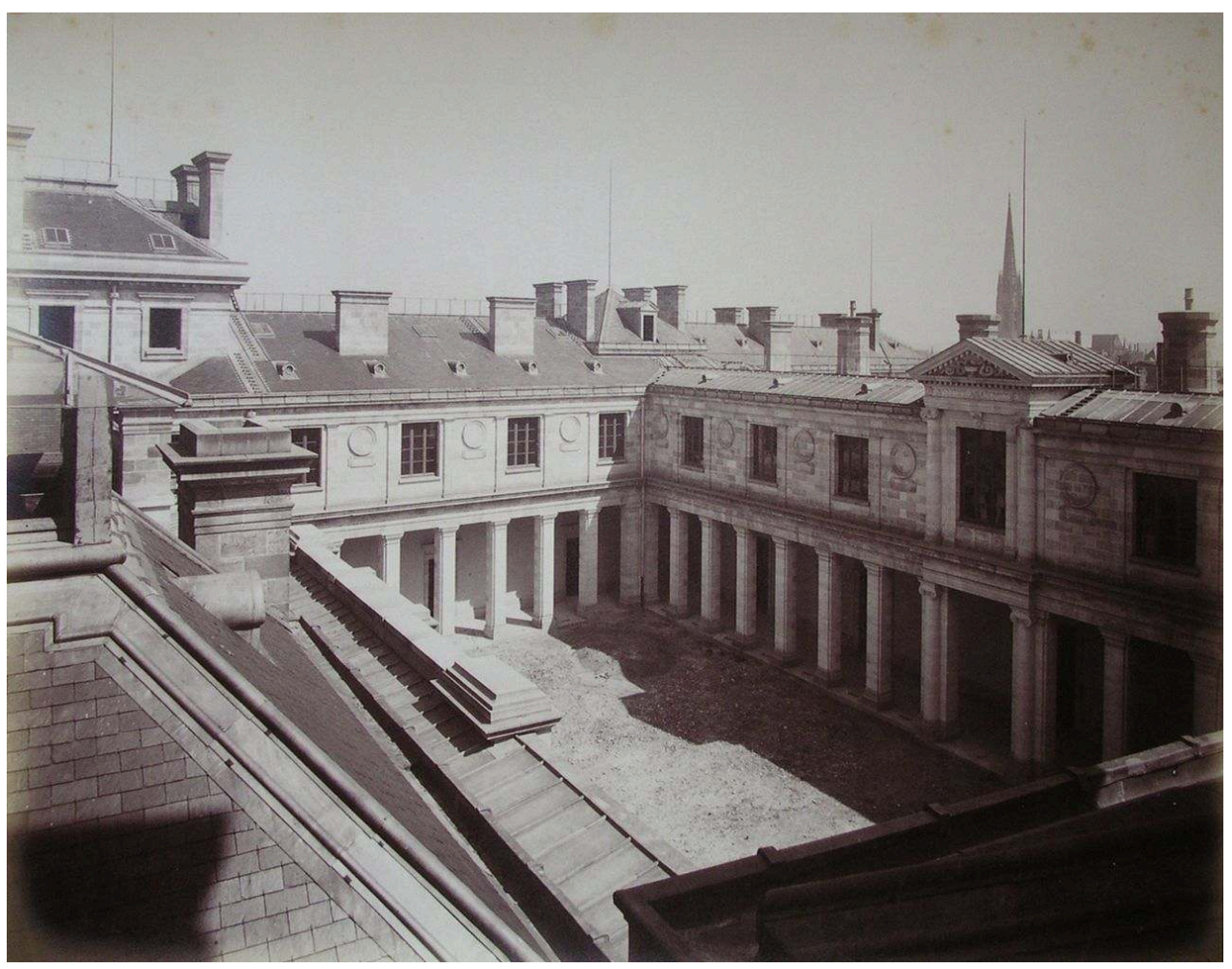

Fig. 5 : Alphonse Terpereau, Faculté de médecine pendant travaux, vers 1886, tirage sur papier albuminé, $36,9 \times 48,2 \mathrm{~cm}$, Bordeaux, archives municipales, 5 M 52 .

Cet état de fait est renforcé par la théâtralisation de ces photographies (fig. 5). Celles-ci sont réalisées par Terpereau qui s'est imposé, depuis les années 1860, comme le premier photographe documentaire en Gironde ${ }^{53}$. Pour les vues d'ensemble de la faculté, il monte sur les toits environnants afin de surplomber l'édifice. Il organise alors ses compositions selon deux points de vue. Pour les grands axes du bâtiment, il cadre de manière frontale afin d'obtenir de puissants raccourcis. Pour les vues d'ensemble, il 
crée des compositions autour des cours intérieures qui forment sur l'image un losange s'inscrivant dans le rectangle de la photographie. Ces effets sont accentués par de puissants effets de clair-obscur qui accentuent l'aspect dramatique de la scène. Il joue ainsi sur la géométrisation des formes et la compression de l'espace qui constituent une part de la modernité visuelle à cette époque ${ }^{54}$.

En ce qui concerne la faculté des sciences, Durand fait lui aussi appel à Terpereau. Le photographe livre le même type d'image concernant le chantier auquel s'ajoutent des vues de l'édifice achevé, ce qui suppose un usage différent. Tout d'abord, les photographies $d u$ chantier sont moins nombreuses. Les archives municipales conservent ainsi cinq vues du chantier et une vue des modèles sculptés. L'architecte et les artisans étant localisés à Bordeaux, la production de ces images n'est plus justifiée. Contrairement à la faculté de médecine, une seule photographie du chantier est datée ${ }^{55}$. Les autres perdent leur valeur documentaire pour ne garder que l'aspect théâtral de la série.

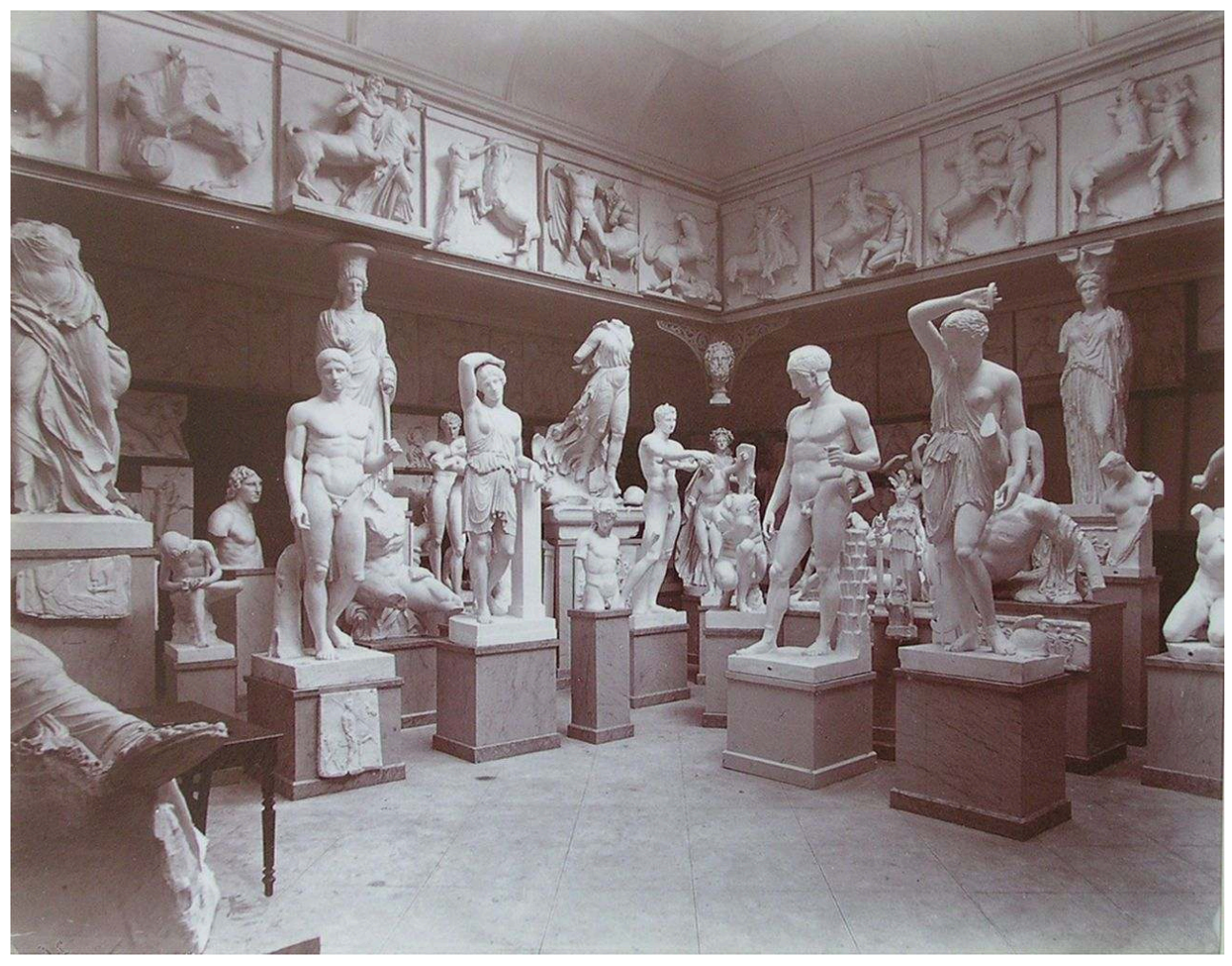

Fig. 6 : Alphonse Terpereau, Musée archéologique de la faculté des sciences, vers 1886, tirage sur papier albuminé, 19,9 × 26,1 cm, Bordeaux, bibliothèque municipale, $\mathrm{H} 87$.

Autre différence, les douze vues de l'édifice en voie d'achèvement sont remplacées par dix-neuf vues de l'édifice achevé avec le mobilier. L'accent est donc mis sur l'équipement. Terpereau livre ainsi à la mairie des vues des amphithéâtres, des salles de cours, des bibliothèques, des laboratoires et des collections (fig. 6) ${ }^{56}$. Des doubles de ces images ont été assemblés avec les vues des chantiers et reliés sous la forme d'albums prestigieux. Ces albums sont conservés par la mairie et le rectorat et ont été présentés aux Expositions universelles de Paris (1889) $)^{57}$ et de Chicago (1893) ${ }^{58}$ par le ministère de l'Instruction publique. Elles représentent alors la modernité de l'enseignement et donc la puissance retrouvée de la nation. 
Les chantiers des facultés révèlent ainsi une standardisation des images qui répond à de multiples usages. La rhétorique visuelle qui fait apparaître la figure de l'échafaudage, désormais habituelle lors des grands travaux, rappelle celle mise en place par les ingénieurs dès la fin des années 1850. Dans les années 1880, les imageries des architectes et des ingénieurs tendent à se confondre. La photographie devient l'illustration d'un monde technique et scientifique marqué par l'idée de progrès et de performance. L'usage de photographies peut alors glisser du simple contrôle ou de la correspondance administrative à l'illustration d'un discours fortement connoté.

\section{Conclusion}

La photographie produite par les architectes au XIX ${ }^{\mathrm{e}}$ siècle à Bordeaux trouve donc son origine, avec les travaux de Paul Abadie, dès la fin des années 1850. Elle témoigne des nouveaux usages liés à l'architecture historiciste, mais aussi de nouvelles pratiques administratives telles que la multiplication des contrôles et l'augmentation de la correspondance. Très vite, à partir de 1865 , cette imagerie se double de celle des ingénieurs issus des services municipaux. L'accent est mis sur la théâtralisation des vues et la création de figures nouvelles qui sont largement diffusées, tels que l'échafaudage ou le chantier. Vers 1880, la photographie d'architecture, qu'elle soit produite par les architectes ou les ingénieurs, tend à se standardiser sous la forme de séries restituant les différentes phases des travaux. Elle est utilisée dans un but administratif mais aussi démonstratif. Récupérée par les services municipaux, elle est stockée dans le portefeuille des Travaux publics. Elle illustre alors le discours des politiques et contribue à la conquête et à la conservation du pouvoir.

Cette histoire se prolonge tout au long $\mathrm{du} \mathrm{xx}^{\mathrm{e}}$ siècle avec la construction de la Bourse $\mathrm{du}$ Travail sous le Front populaire ou celle de la base sous-marine sous l'occupation nazie. Elle connaitra de nouvelles dynamiques avec la création des grands ensembles d'aprèsguerre puis avec la création par Jacques Chaban-Delmas en 1986 de la Mémoire de Bordeaux, organisme patrimonial qui assure, parallèlement aux archives municipales, la production et le stockage d'archives photographiques et cinématographiques concernant les grands travaux effectués lors de ses différents mandats, et enfin avec Alain Juppé et le projet urbain « Bordeaux 2030 » marqué par les techniques de création et de diffusion numériques ${ }^{59}$.

\section{BIBLIOGRAPHIE}

Barry BERGDOLL, « Dudan et la photograhie » dans Sylvain BELLANGER et Françoise HAMON (dir.), Duban. Les couleurs de l'architecte, Paris, Gallimard/Electra, 1996, p. 228-232.

Marc FAVREAU, Les " portraits » de Bordeaux. Vues et plans gravés de la capitale de la Guyenne du XVI et XVIII ${ }^{e}$ siècle, Camiac-et-Saint-Denis, Édition de l'Entre-deux-Mers, 2007. 
André GUNTHERT, « Le rêve de l'architecte », L'Atelier des icônes, 12 août 2012 [consulté le 28 janvier 2016].

Marion LAGRANGE et Florent MIANE, «Le Musée archéologique de la faculté des lettres de Bordeaux (1886).L'institutionnalisation des collections pédagogiques et scientifiques », In Situ, $\mathrm{n}^{\circ} 17$ [consulté le 28 janvier 2016].

Claude LAROCHE, « Pro Scientia Urbe et Patria : L'architecture de la faculté de médecine et de pharmacie de Bordeaux, 1876-1888 et 1902-1922 »,In Situ, n 17, 2011 [consulté le 28 janvier 2016].

Claude LAROCHE, Paul Abadie, architecte 1812-1884, Paris, RMN, 1988.

Anne MACCAULEY, Industrial Madness, New Haven, Yale University Press, 1994.

Florent MIANE, Alphonse Terpereau, 1839-1897. Le premier photographe documentaire en Gironde, Bordeaux, Archives départementale de la Gironde, 2012.

Marie-Madeleine OZDOBA, «Images d'architecture contemporaines : un nouveau romantisme », L'Atelier des icônes, 5 mars 2013 [consulté le 28 janvier 2016].

Elvire PEREGO, « Delmaet \& Durandelle ou la rectitude des lignes, un atelier du XIX ${ }^{\mathrm{e}}$ ", Photographies, $\mathrm{n}^{\circ}$ 5, 1984, p. 54-75.

Elvire PEREGO, « La ville-machine. Architecture et industrie » dans Michel FRIZOT, Nouvelle histoire de la photographie, Paris, Bordas/Adam Birot, 1994, p. 197-217.

Pierre-Lin RENIÉ, « De l'imprimerie photographique à la photographie imprimée », Études photographiques, $\mathrm{n}^{\circ} 20$, juin 2007, p. 18-33.

Pierre-Lin RENIÉ, Une image sur un mur, Bordeaux, musée Goupil, 2005.

Anne-Marie de THÉZY, Marville, photographe de Paris, Paris, Hazan, 1999.

\section{NOTES}

1. Barry BERGDOLL, «Dudan et la photograhie » dans Sylvain Bellanger et Françoise HAMON (dir.), Duban. Les couleurs de l'architecte, Paris, Gallimard/Electra, 1996, p. 228-232.

2. Anne Maccauley, Industrial Madness, New Haven, Yale University Press, 1994, p. 195-232.

3. Florent MIANE, Alphonse Terpereau, 1839-1897. Le premier photographe documentaire en Gironde, Bordeaux, Archives départementale de la Gironde, 2012, p. 6-7.

4. Claude LAROCHE, Paul Abadie, architecte 1812-1884, Paris, RMN, 1988, p. 146-149.

5. Bordeaux, archives municipales [AMB], correspondances, 4015 M 4, 21 décembre 1860 : «Ils exposent que l'église $S^{\text {te }}$ Croix est, dans son état actuel, un monument extrêmement rare, un diamant de l'archéologie, mais à condition de rester ce qu'elle est. [...] Il faut respecter la vétusté, les générations n'ont pas le droit d'altérer les monuments qui font l'ornement d'une ville, c'est pour elles un devoir strict que de les transmettre aux générations futures : il faut donc laisser $\mathrm{S}^{\text {te }}$ Croix inachevée, sans grandissement, sans clocher; il faut conserver ce monument comme une vieille ruine devant laquelle on s'incline avec respect. »

6. $\mathrm{AMB}$, délibérations, $12 \mathrm{D} 43,21$ décembre 1860.

7. AMB, correspondances, $4015 \mathrm{M}$ 4, 9 juin 1865.

8. $\mathrm{AMB}$, iconographie, 4 I 89.

9. $\mathrm{AMB}$, correspondances, $4015 \mathrm{M} 4,1^{\mathrm{er}}$ mai 1862 .

10. AMB, correspondances, 4015 M 4, 5 février 1869, exercice de 1862. 
11. AMB, correspondances, $4015 \mathrm{M} \mathrm{4,3}$ novembre 1862 : «j'ai, à l'aide d'instructions successives rétabli la façade de cette église telle qu'elle a été conçue primitivement et j'en ai montré les altérations partielles. »

12. Ibid. : « La Commission après un examen approfondi des diverses transformations subies par la façade de $\mathrm{S}^{\text {te }}$ Croix, après avoir reconstitué dans son esprit cette façade aux formes si inexplicables jusqu'à ce jour, a été conduite à conclure que ce qu'il y avait de plus conforme à la raison, de plus satisfaisant pour l'art, de plus agréable pour les yeux, c'était de revenir à la forme primitive. "

13. AMB, correspondances, 4015 M 4, 29 décembre 1862 « Il existe deux époques, bien marquées, la façade actuelle, celle que les archéologues admirent est un placage sur la façade primitive ; cette assertion aujourd'hui ne peut être contestée après l'inspection attentive des lieux. »

14. AMB, correspondances, $4015 \mathrm{M} \mathrm{4,10}$ août 1864 .

15. AMB, correspondances, 4015 M 4, 8 septembre 1864 : "Quant aux dessins ce n'est plus possible. La plupart de nos statuaires ne savent pas dessiner. »

16. AMB, correspondances, $4015 \mathrm{M}$ 4, 9 juin 1865.

17. AMB, correspondances, $4015 \mathrm{M}$ 4, 20 juin 1865.

18. Ibid. : " C'est donc M. Abadie, et non M. Pascal, qui est obligé de puiser aux bonnes sources, de faire exécuter des estampages ou des photographies. Il s'agit d'une restauration très difficile et qui exige une prudence extrême dans les choix des renseignements. A M. Abadie seul était réservé ce soin et les honoraires qui lui sont alloués ne sont gagnés par lui qu'à cette condition ».

19. AMB, iconographie, 4 I 10 ; 4 I 97.

20. Paris, Bibliothèque nationale de France [BnF], dépôt de Terpereau, EO 137 (14-1).

21. Pierre-Lin RENIÉ, "De l'imprimerie photographique à la photographie imprimée », Études photographiques, $n^{\circ} 20$, juin 2007, p. 18-33.

22. Pierre-Lin RENIÉ, Une image sur un mur, Bordeaux, musée Goupil, 2005, p. 7-15.

23. Paris, Bibliothèque de l'Institut de France [IDF], album Abadie, fo $\mathrm{X} 276$.

24. Claude LAROCHE, op. cit., p. 138-145.

25. Le dessin original se trouve conservé dans le fonds iconographique des archives municipales de Bordeaux sous la cote 5 L 204.

26. AMB, correspondances, 3040 M 1, 7 janvier 1857.

27. AMB, correspondances, 3040 M 1, s. d.

28. Anne-Marie de THÉZY, Marville, photographe de Paris, Paris, Hazan, 1999, n. p.

29. Marc FAVREAU, Les " portraits » de Bordeaux. Vues et plans gravés de la capitale de la Guyenne du XVI et XVIII siècle, Camiac-et-Saint-Denis, Édition de l'Entre-deux-Mers, 2007, p. 148-161. La tour est représentée sur le plan de la ville dit de Lattré, gravé en 1755.

30. AMB, iconographie, 5 L 35 ; 5 L 164 ; 5 L 165 ; 5 L 166 ; 5 L 167.

31. Le relevé d'Abadie est conservé dans le fonds iconographique des archives municipales de Bordeaux sous la cote $5 \mathrm{~L} 203$.

32. AMB, correspondances, $3040 \mathrm{M} \mathrm{3,} 13$ août 1863.

33. AMB, correspondances, 3040 M 4, 8 septembre 1863 : «M. Abadie s'aperçut que les sommiers de ces arcs, inhabilement appareillés, s'étant brisés dès l'origine, circonstance à laquelle il attribue la construction du mur qui fermait les grandes ouvertures du rez-de-chaussée. »

34. Bordeaux, Archives départementales de la Gironde [ADG], grands formats, 3 Z 5. Le dessin est daté du $1^{\mathrm{er}}$ septembre 1863.

35. AMB, correspondances, $3040 \mathrm{M} \mathrm{1,} 21$ octobre 1863.

36. AMB, correspondances, $3040 \mathrm{M} \mathrm{1,} 1^{\text {er }}$ décembre 1863 : «Bien que les travaux projetés soient indispensables, la demande des crédits nécessaires à leur exécution soulèvera probablement une vive discussion dans le sein du Conseil municipal, j'ai pensé que dans la situation des choses, l'administration obtiendrait plus facilement un vote favorable si le projet présenté était approuvé par la Commission des monuments historiques. » 
37. AMB, correspondances, 3040 M 5, 9 novembre $1865 ; 29$ novembre 1865 .

38. AMB, iconographie, 5 L 010 ; 5 L 158 ; 5 L 159 ; 5 L 160 ; 5 L 161 ; 5 L 162 ; 5 L 163 (flèche).

39. Bordeaux, bibliothèque municipale de Bordeaux [BMB], fonds Delpit, XVI 51-1; XVI 51-2 (la flèche) ; XVI 51-3 ; XVI 51-4 (la tour) ; XVI 52-1 (la base).

40. ADG, albums de la Commission, $162 \mathrm{~T} 8$ (la flèche).

41. AMB, correspondances, $3040 \mathrm{M} 2$ : le 28 novembre 1865, la mairie verse au photographe 90 francs pour la livraison de 30 épreuves ; 3040 M 5 : le 7 février 1866, elle verse une somme de 30 francs pour dix nouvelles épreuves.

42. BMB, iconographie, XVI 51-3 ; XVI 51-4 ; XVI 52-1 ; BnF, iconographie, EO 137 a (06)

43. BnF, iconographie, EO 137 a (05).

44. AMB, iconographie, 05 L 003 ; BMB, iconographie, XVI 52-2 ; BnF, iconographie, EO 137 (26).

45. IDF, album Abadie, $\mathrm{f}^{\circ} \mathrm{X} 276, \mathrm{f}^{\circ} 7$ pour la tour achevée.

46. Claude LAROCHE, "Pro Scientia Urbe et Patria : L'architecture de la faculté de médecine et de pharmacie de Bordeaux, 1876-1888 et 1902-1922 »,In Situ, nº 17 [consulté le 28 janvier 2016]. La réalisation de la faculté de médecine est attribuée par concours à l'architecte Pascal, Prix de Rome en 1866. Il remplace Henri Labrouste sur le chantier de la Bibliothèque Nationale à la mort de ce dernier en 1875. La faculté des sciences est confiée sans concours à l'architecte municipal Charles Durand.

47. AMB, correspondances, $6872 \mathrm{M} 26,30$ mars 1886.

48. AMB, correspondances, $6872 \mathrm{M} 26,26$ janvier 1894.

49. Construction de la faculté de médecine de Bordeaux / Situation des travaux.

50. Par exemple pour les douze premières : AMB, iconographie, 5 M $23:$ « 29 décembre 1880 », 5

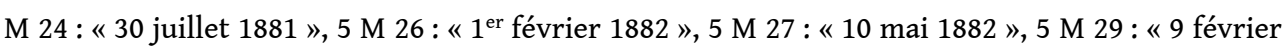
1883 », $5 \mathrm{M} 31$ : « 13 juin 1883/première vue », $5 \mathrm{M} 34:$ « 13 juin 1883/deuxième vue », $5 \mathrm{M} 37$ : « 13 juin 1883/troisième vue », $5 \mathrm{M} 40:$ « 13 juin 1883/quatrième vue », $5 \mathrm{M} 43$ : « 24 mars 1884/ première vue », 5 M $45:$ « 24 mars 1884/deuxième vue », 5 M 47 : « Février 1885 ».

51. ADG, fonds Pascal, 212 T 70, 5 avril 1890.

52. AMB, correspondances, $6872 \mathrm{M} \mathrm{26,29}$ mars 1886.

53. Après avoir travaillé pour l'architecte Abadie et pour la Commission des monuments historique dans les années 1860, Terpereau reçoit des commandes de la part de nombreux ingénieurs notamment Stanislas de Laroche-Tolay lors de la construction des bassins à flot de Bordeaux (1872-1877) et Gustave Eiffel lors de la construction du viaduc de Garabit (1882-1886).

54. Elvire PEREGO, "Delmaet \& Durandelle ou la rectitude des lignes, un atelier du $\mathrm{XIX}^{\mathrm{e}}$ ", Photographies, $n^{\circ}$ 5, 1984, p. 54-75 et «La ville-machine. Architecture et industrie » dans Michel FRIZOT, Nouvelle histoire de la photographie, Paris, Bordas/Adam Birot, 1994, p. 197-217.

55. AMB, iconographie, $08 \mathrm{R}$ 189, 21 avril 1882.

56. Marion LAGRANGE et Florent MIANE, «Le Musée archéologique de la faculté des lettres de Bordeaux (1886).L'institutionnalisation des collections pédagogiques et scientifiques ", In Situ, $\mathrm{n}^{\circ} 17$ [consulté le 28 janvier 2016].

57. Bordeaux, archives du rectorat de Bordeaux [ARB], conseil général des facultés de Bordeaux, $\mathrm{R} 23$, séance du 28 février $1888, \mathrm{f}^{\circ} 52$.

58. ARB, conseil général des facultés de Bordeaux, $R$ 23, séance du 29 novembre 1892 , fo 184 .

59. André GUNTHERT, "Le rêve de l'architecte ", L'Atelier des icônes, 12 août 2012, et MarieMadeleine OZDOBA, «Images d'architecture contemporaines : un nouveau romantisme », L'Atelier des icônes, 5 mars 2013 [consultés le 28 janvier 2016]. 
INDEX

Index géographique : Bordeaux, Gironde

Mots-clés : Architecture, Cathédrale, conservation-restauration, historiens et architectes, Monument, patrimoine architectural et urbain, photographie, restauration

Index chronologique : XIXe siècle

AUTEUR

FLORENT MIANE

Maître de conférences en histoire de l'art contemporain. Université de Bretagne Occidentale 